

\section{PREDICTION OF LIMIT CYCLE PRESSURE OSCILLATIONS IN GAS TURBINE COMBUSTION SYSTEMS USING THE FLAME DESCRIBING FUNCTION}

Harmen Krediet 


\section{Composition of the graduation committee:}

Chairman and secretary:

Prof. dr. F. Eising University of Twente

Promoter:

Prof. dr. ir. Th.H. van der Meer University of Twente Assistant promoter:

Dr. ir. J.B.W. Kok University of Twente Members:

Prof. dr. D.J.E.M. Roekaerts

Delft University of Technology

Prof. dr. ir. A. de Boer

University of Twente

Prof. dr. ir. A. Hirschberg

Prof. dr. ir. B.J. Geurts

Dr. W. Krebs

University of Twente

University of Twente

Siemens Energy

This research was financially supported by the European Commission in the Marie Curie Actions - Networks for Initial Training program, under call FP7-PEOPLE-2007-1-1-ITN, Project LIMOUSINE, with project number 214905.

(C) Harmen Krediet, Zwolle, 2012

No part of this publication may be reproduced by print, photocopy or any other means without the permission of the copyright owner.

ISBN: 978-90-365-3372-0

DOI: $10.3990 / 1.9789036533720$

Printed by: Proefschriftmaken.nl || Printyourthesis.com

Published by: Uitgeverij BOXPress, Oisterwijk

Keywords: Thermo-acoustics, Combustion Instability, Limit cycle, Gas turbine, Flame Describing Function, Large Eddy Simulation 


\title{
PREDICTION OF LIMIT CYCLE PRESSURE OSCILLATIONS IN GAS TURBINE COMBUSTION SYSTEMS USING THE FLAME DESCRIBING FUNCTION
}

\section{DISSERTATION}

\author{
to obtain \\ the degree of doctor at the University of Twente, \\ on the authority of the rector magnificus, \\ prof. dr. H. Brinksma, \\ on account of the decision of the graduation committee, \\ to be publicly defended \\ on Friday the $29^{\text {th }}$ of June 2012 at 12:45h \\ by \\ Harmen Jan Krediet \\ born on May 23, 1984 \\ in Warnsveld, the Netherlands
}


This dissertation has been approved by:

The promoter:

The assistant promoter:
Prof. dr. ir. Th.H. van der Meer

Dr. ir. J.B.W. Kok 


\section{Summary}

In order to meet low $N O_{X}$ emission targets for modern gas turbines used for power generation, lean premixed combustion needs to be applied. A drawback of this combustion technology is that these combustion systems are very prone to thermoacoustic instabilities, which arise from the interactions between the acoustics of the combustion system and the heat release of the flame. These thermo-acoustic instabilities can lead to significant sound pressure levels in the combustion chamber, which may cause severe damage to the gas turbine. Conditions with high sound pressure levels should therefore be avoided at all costs. Therefore, it is desirable to predict the limit cycle pressure amplitude during the design phase of a gas turbine combustor, to determine under which conditions stable operation can be achieved.

The work presented in this dissertation focuses on the numerical prediction of thermo-acoustic instabilities and limit cycle pressure oscillations. The predictions are based on Computational Fluid Dynamics (CFD) simulations.

In general, there are two key strategies for studying thermo-acoustic instabilities using CFD. The first strategy is the self-excited method. In a self-excited CFD simulation, the simulation becomes unsteady by itself and develops a limit cycle. In order to obtain self-excited pressure oscillations, a computational grid of the complete combustion system is made, ranging from compressor outlet to turbine inlet, so that the boundary conditions of the CFD match the physical boundary conditions.

The second approach, which is investigated in this work, is the forced response method. In the forced response method, only a part of the combustion system, including the burner and the combustion chamber, is modeled. The flame is willing excited by superimposing an acoustic wave on the inlet mass flow rate and from the flame response, a transfer function between acoustic velocity oscillations and heat release oscillations is determined. This transfer function is dependent on the frequency and amplitude of the acoustic waves and is called the Flame Describing Function. The Flame Describing Function is used as an input in an one-dimensional acoustic code, which models the complete combustion system. Using this low-order acoustic code, the limit cycle pressure amplitude is predicted.

The first part of this dissertation deals with the development of such a onedimensional acoustic code. A nonlinear thermo-acoustic model for the prediction of limit cycles is proposed and validated with data found in literature. Different lab scale combustion systems are studied. For the validation of the model, Flame Describing Functions obtained from available measurements are used. For all operating points and configurations which have been modeled, the linear stability of the combustion system is successfully predicted. For the unstable points, the limit cycle pressure amplitudes are successfully predicted as well. 
In the next part of this dissertation, it is investigated if the Flame Describing Function can be predicted from CFD simulations. In this case, Large Eddy Simulations are performed. The test case used is a generic atmospheric swirl flame, for which a perfectly premixed and a partially premixed operating point have been studied. For both operating points, the nonlinear response of the heat release, which was observed during measurements, was captured by the LES. It is found that the magnitude of the Flame Describing Function reduces with larger excitation amplitudes, which means that the heat release saturates.

Next the Flame Describing Function from the CFD simulations was used together with the one-dimensional acoustic code to predict the limit cycle pressure amplitude. For the partially premixed case, the frequency was predicted within $8 \mathrm{~Hz}$, the pressure amplitude within $3.3 \mathrm{~dB}$ compared to measurements. For the perfectly premixed case a limit cycle pressure amplitude was predicted as well. However, this operating point appeared to be stable in measurements. It was found that this result is caused by the fact that the phase of the Flame Describing Function was under predicted in the simulations by $0.4 \pi$. A parametric analysis showed that changes of the phase of less than $10 \%$ of the period can double the limit cycle amplitude.

In addition, the reflection coefficient appeared to have a major impact on the predicted limit cycle amplitude as well. This shows that the limit cycle can only predicted accurately when both accurate data on the FDF and acoustic energy losses are available. It can also be concluded that the results from a nonlinear limit cycle calculation are only relevant when this sensitivity is taken into account, since changes may be large for small changes in time lag and or boundary conditions.

After validating the approach for generic combustion systems, also the Flame Describing Function and limit cycle pressure amplitude of an industrial combustion system, are predicted. The previous FDF's that have been determined were of the Single Input, Single Output type, which means that the heat release model takes only into account velocity perturbations. For this configuration a Multiple Input, Single Output heat release model is determined, which takes into account both velocity fluctuations and equivalence ratio fluctuations.

Also for this configuration it is found that the magnitude of the Flame Describing Function depended on the excitation amplitude, indication saturation of the heat release rate. Using the one-dimensional acoustic code, the amplitude from the simulations is predicted within $4.8 \mathrm{~dB}$ and the frequency in the within $6 \%$, compared to the measurements.

Using the MISO model, the individual contribution of equivalence ratio fluctuations and velocity fluctuations is analyzed in more detail. It is found that velocity fluctuations excite the thermo-acoustic instabilities, whereas equivalence ratio fluctuations damp them. This result is then used to propose a design change that improves the thermo-acoustic stability behavior of the burner. It is found that the stability of the burner could be improved by shifting the location of the fuel injection downstream, towards the flame. It would not have been possible to identify the position of the fuel injectors as an important design parameter using a SISO model. This analysis therefore demonstrates the additional benefit of MISO models. 


\section{Samenvatting}

Om lage emissies van $N O_{X}$ te kunnen realiseren in moderne gasturbines, gebruikt voor de productie van elektriciteit, moet magere voorgemengde verbranding worden toegepast. Een nadeel van deze verbrandingstechnologie is dat dergelijke verbrandingssystemen zeer gevoelig zijn voor thermo-akoestische instabiliteiten, die ontstaan door de interacties tussen de akoestiek van het verbrandingssysteem en de warmteproductie van de vlam. Deze thermo-akoestische instabiliteiten kunnen tot grote geluidsdrukken in de verbrandingskamer leiden en kunnen de gas turbine zwaar beschadigen. Om deze reden moeten condities met hoge geluidsdrukken ten aller tijden vermeden worden. In de ontwerpfase van een gasturbine is het daarom wenselijk om de grenscyclus druk amplitudes in de verbrandingskamer te kunnen voorspellen, om te bepalen onder welke condities de gas turbine stabiel bedreven kan worden.

Het werk dat in dit proefschrift gepresenteerd wordt richt zich op het numeriek voorspellen van thermo-akoestische instabiliteiten en grenscyclus druk amplitudes. De voorspellingen zijn gebaseerd op Computational Fluid Dynamics (CFD) simulaties.

Over het algemeen zijn er twee strategieën om thermo-akoestische instabiliteiten te kunnen bestuderen met CFD. De eerste strategie is de "self-excited" methode. Met deze methode wordt de simulatie zelf onstabiel en wordt er een grenscyclus ontwikkeld. Om zelfopgewekte druk oscillaties te kunnen opwekken, moet er een rekengrid van het complete verbrandingssysteem gemaakt worden. De gehele geometrie tussen de uitlaat van de compressor en de inlaat van de turbine moet meegenomen worden, zodat de randvoorwaarden van de CFD simulatie met de fysische randvoorwaarden overeenkomen.

De tweede methode, welke in deze studie onderzocht wordt, is de "forced response" methode. In de forced response methode wordt slechts een gedeelte van het verbrandingssysteem, in ieder geval de brander en de verbrandingskamer omvattende, gemodelleerd. De vlam wordt gepertubeerd door een akoestische golf op de massastroom randvoorwaarde bij de inlaat te superpositieneren en de responsie van de vlam tengevolge van deze akoestische golf wordt bepaald. Zo kan een overdrachtsfunctie tussen de oscillaties van de akoestische snelheid en de warmteproductie van de vlam gevonden worden. Deze overdrachtsfunctie is afhankelijk van de frequentie en amplitude van de akoestische golven en wordt in dit proefschrift de "Flame Describing Function" genoemd. De Flame Describing Function (FDF) wordt als invoer in een eendimensionale akoestische code gebruikt, welk het complete verbrandingssysteem modelleert. Met deze code worden dan uiteindelijk de grenscyclus drukamplitudes voorspeld. 
Het eerste deel van dit proefschrift gaat over het opzetten van een dergelijke eendimensionale code. Een niet-lineaire thermo-akoestische code voor het voorspellen van grenscycli wordt voorgesteld en gevalideerd met data gevonden in de literatuur. Verschillende verbrandingssystemen op laboratoriumschaal worden bestudeerd. Voor het valideren van het model worden Flame Describing Functions verkregen uit beschikbare metingen gebruikt. Voor alle bedrijfspunten en configuraties die gemodelleerd zijn wordt de lineaire stabiliteit succesvol voorspeld. Voor de instabiele bedrijfspunten wordt ook de grenscyclus druk amplitude succesvol voorspeld.

In het tweede deel van dit proefschrift wordt onderzocht of de Flame Describing Function met CFD simulaties voorspeld kan worden. In dit geval wordt Large Eddy Simulatie gebruikt. De test case is een generieke atmosferische swirl vlam, waarvoor een perfect voorgemengd en een gedeeltelijk voorgemengd bedrijfspunt bestudeerd is. Voor beide bedrijfspunten werd de niet-lineaire responsie van de warmteproductie van de vlam, die in metingen waargenomen is, ook met LES gevonden. Er wordt gevonden dat de amplitude van de Flame Describing Function kleiner wordt bij grotere pertubatie amplitudes, wat betekent dat de warmteproductie van de vlam verzadigt.

Vervolgens wordt de Flame Describing Function uit de CFD simulaties samen met de eendimensionale akoestische code gebruikt om de grenscyclus druk amplitude te voorspellen. Voor het gedeeltelijk voorgemengde bedrijfspunt wordt de frequentie binnen $8 \mathrm{~Hz}$ voorspeld en de drukamplitude binnen $3.3 \mathrm{~dB}$ in vergelijking met metingen. Voor het perfect voorgemengde bedrijfspunt werd echter ook een instabiliteit voorspeld. Dit bedrijfspunt bleek in metingen stabiel te zijn. De reden van deze tegenstrijdigheid bleek het feit te zijn dat de fase van de Flame Describing Function in de CFD simulaties met $0.4 \pi$ onderschat werd. Uit een sensitiviteitsanalyse blijkt dat veranderingen in de fase van minder dan $10 \%$ van de periode de grenscyclus drukamplitude kunnen verdubbelen.

Daarnaast blijkt ook de reflectiecoëfficiënt een belangrijke invloed op de voorspelde drukamplitude te hebben. Dit laat zien dat de grenscyclus alleen accuraat voorspeld kan worden als betrouwbare data voor de FDF en akoestische energie verliezen beschikbaar zijn. Er kan ook geconcludeerd worden dat de resultaten van een niet-lineaire grenscyclus berekening alleen relevant zijn wanneer met deze sensitiviteit rekening gehouden wordt, aangezien grote veranderingen in de drukamplitude gevonden worden voor kleine veranderingen in de fase en reflectiecoëfficiënt.

Na dit validatiewerk voor generieke verbrandingssystemen, wordt ook de Flame Describing Function en grensccyclus drukamplitude voor een industrieel verbrandingssysteem voorspeld. De FDF's die tot nu toe bepaald werden zijn van het "Single Input, Single Output" type, hetgeen betekent dat het vlammodel alleen akoestische snelheidsfluctuaties betracht. Voor deze configuratie wordt een Multiple Input, Single Output overdrachtsfunctie bepaald, welke zowel snelheidsfluctuaties als equivalentieratio fluctuaties meeneemt.

Ook voor deze configuratie wordt gevonden dat de amplitude van de Flame Describing Function afhankelijk is van de pertubatie amplitude, wat verzadiging van de warmteproductie betekent. Met de eendimensionale akoestische code wordt de frequentie van de grenscyclus binnen $6 \%$ voorspeld en de amplitude binnen $4.8 \mathrm{~dB}$, 
vergeleken met metingen.

Met het MISO model worden de individuele contributies van snelheids en equivalentieratio fluctuaties in meer detail geanalyseerd. Er wordt gevonden dat de snelheidsfluctuaties de thermo-akoestische instabiliteit opwekken, waarentegen equivalentieratio fluctuaties deze dempen. Dit resultaat wordt dan gebruikt om een ontwerpverandering voor te stellen dat het thermo-akoestische stabiliteitsgedrag van de brander verbetert. Door de positie waar de brandstof geïnjecteerd wordt meer richting de vlam te verschuiven wordt de stabiliteit van het verbrandingssysteem verbeterd. Met een SISO model zou niet geïdentificeerd zijn dat de positie van de brandstofinjectie zo' $n$ belangrijke ontwerpparameter is. Deze analyse laat daarom de toegevoegde waarde van MISO modellen zien. 


\section{Contents}

Summary $\quad$ v

Samenvatting vii

1 Introduction 1

1.1 Motivation . . . . . . . . . . . . . . . . . . 1

1.2 Thermo-acoustic instabilities: literature review . . . . . . . . . . . 2

1.2.1 Control of thermo-acoustic instabilities . . . . . . . . . . 3

1.2.2 Modeling strategies of thermo-acoustic instabilities . . . . . . . 5

1.2.3 The Flame Describing Function . . . . . . . . . . . . . . . 7

1.2.4 Perfectly versus partially premixed flames . . . . . . . . . . . . 10

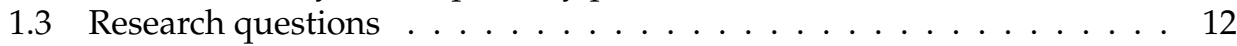

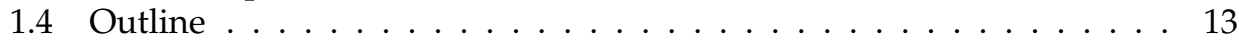

2 Thermo-acoustic instability model 15

2.1 Introduction . . . . . . . . . . . . . . . . 15

2.1.1 Overview of existing models . . . . . . . . . . . . . 15

2.1.2 Extension to a nonlinear instability model . . . . . . . . . . . . 17

2.2 Model formulation . . . . . . . . . . . . . . . . . 17

2.2.1 Governing equations . . . . . . . . . . . . . . . . 18

2.2.2 Expansion as Galerkin series . . . . . . . . . . . . . . . . 19

2.2.3 Treatment of boundary conditions ... . . . . . . . . . 20

2.2.4 Treatment of heat release rate . . . . . . . . . . . . . . 21

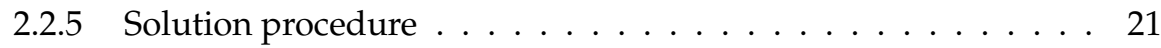

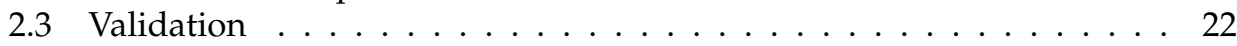

2.3.1 Bluff body stabilized flame . . . . . . . . . . . . . 22

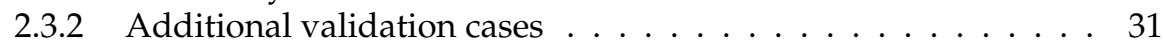

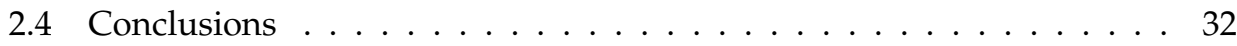

3 Transient CFD simulation of the reacting flow 35

3.1 Introduction . . . . . . . . . . . . . . . 35

3.2 Equations governing turbulent reacting flow . . . . . . . . . . 36

3.2 .1 Introduction to LES . . . . . . . . . . . . . . . . . . . 38

3.2.2 LES Filtered Navier Stokes equations for turbulent reacting flow 38

3.2.3 Turbulence model . . . . . . . . . . . . . . . . . . . 40

3.2.4 Combustion model . . . . . . . . . . . . . . . . . 41

3.2.5 Final equation set implemented in the reacting LES code . . . . 45 
3.2.6 Numerical simulation . . . . . . . . . . . . . . . . . 46

3.3 CFD simulation of thermo-acoustic systems . . . . . . . . . . . . 46

3.3.1 Boundary conditions . . . . . . . . . . . . . . . 47

3.3.2 Sampling of acoustic quantities . . . . . . . . . . . . . . 48

3.3.3 Postprocessing of thermo-acoustic response . . . . . . . . . . . 49

3.4 Verification for thermo-acoustic applications . . . . . . . . . . . . 51

3.4.1 Propagation of a 1D acoustic wave . . . . . . . . . . . 52

3.4 .2 High amplitude boundary excitation . . . . . . . . . 53

3.5 Conclusions . . . . . . . . . . . . . . . . . . 57

4 Application to a generic atmospheric swirl flame 59

4.1 Introduction . . . . . . . . . . . . . . . 59

4.2 Description of test case . . . . . . . . . . . . . . . 60

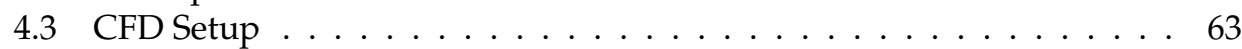

4.4 Steady flow field results . . . . . . . . . . . . . . . . 65

4.5 Flame Describing Function from CFD . . . . . . . . . . . . . 68

4.5.1 Perfectly premixed case . . . . . . . . . . . . . . 69

4.5.2 Partially premixed case ....................... 73

4.6 Investigation of the saturation mechanism . . . . . . . . . . . . 74

4.7 Nonlinear stability analysis with the Generalized Instability Model . . 80

4.7 .1 Model setup . . . . . . . . . . . . . . . . 80

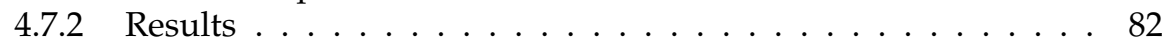

4.8 Self-excited simulation . . . . . . . . . . . . . . . . 87

4.8.1 Comparison with forced response approach . . . . . . . . . . 91

4.9 Conclusions ........................... 91

5 Application to a high pressure multi-jet flame combustor 93

5.1 Introduction . . . . . . . . . . . . . . . . 93

5.2 Description of test case and computational domain . . . . . . . . . . 94

5.3 Steady flow field results . . . . . . . . . . . . . . . 96

5.4 Results of the simulation with excitation . . . . . . . . . . . . . . 98

5.4.1 Postprocessing algorithm . . . . . . . . . . . . . . . . 98

5.4 .2 Flame Describing Function . . . . . . . . . . . . . . . . 100

5.5 Nonlinear stability analysis . . . . . . . . . . . . . . . . . . . . 102

5.5 .1 Model setup . . . . . . . . . . . . . . . . . 102

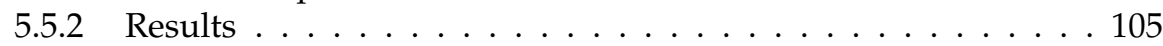

5.5 .3 Analysis of the MISO Model . . . . . . . . . . . . . . . . . 107

5.6 Conclusions . . . . . . . . . . . . . . . . . . 109

6 Conclusions and recommendations 111

6.1 Answering the research questions . . . . . . . . . . . . . . 111

6.2 Recommendations ....................... 115

$\begin{array}{ll}\text { A The Transfer Matrix Approach } & 117\end{array}$

B Generalized Instability Model: derivation of forcing term 123 
C LODI Equations 127

C.1 Inflow boundary condition without excitation . . . . . . . . . . 128

C.2 Inflow boundary condition with excitation . . . . . . . . . . . . . 129

C.3 Outflow boundary conditon . . . . . . . . . . . . . . . . 130

D Transfer function of an area discontinuity 131

E The Wiener Hopf Transformation 137

E.1 Determining the filter length . . . . . . . . . . . . . . . . . 139

F Comparison AVBP and OpenFOAM results 141

F.1 Comparison CFD Setup . . . . . . . . . . . . . . . . . . . . 141

F.2 Steady flow field results . . . . . . . . . . . . . . . . . . 141

F.3 Flame Transfer Function . . . . . . . . . . . . . . . . . . . . 143

G High amplitude multi-frequency excitation $\quad 151$

H TMA model of the swirl stabilized combustor $\quad 155$

$\begin{array}{llr}\text { I Specification of CPU resources } & 159\end{array}$

$\begin{array}{ll}\text { Nomenclature } & 161\end{array}$

$\begin{array}{ll}\text { Publications } & 165\end{array}$

$\begin{array}{ll}\text { Bibliography } & 174\end{array}$

$\begin{array}{ll}\text { Acknowledgements } & 175\end{array}$ 


\section{1 \\ Introduction}

\subsection{Motivation}

In order to meet low $N O_{X}$ emission targets for modern gas turbines used for power generation, lean premixed combustion technology is generally applied. A drawback of this combustion technology is that the resulting high power load makes these gas turbines prone to thermo-acoustic oscillations, which may cause severe damage to the combustion system. Conditions with high pressure oscillations should therefore be avoided at all costs, but as a result the operational envelope of these engines is limited.

An example of a modern gas turbine engine, the Siemens SGT5-8000H, can be seen in figure 1.1. The working of the gas turbine is as follows: Air at atmospheric conditions enters the gas turbine (1) and is compressed in the compressor section (2). Subsequently, the pressurized air enters the burner, in which fuel (usually natural gas) is premixed with the air. The mixture is combusted in the combustion chamber (3) at constant pressure. In the turbine (4), the hot gases are expanded to atmospheric conditions. The resulting power output of the turbine is used to drive the compressor and a generator. Finally the remaining energy in the exhaust gases (5) could be used to produce steam in a steam turbine.

The focus in this dissertation is on the thermo-acoustic instabilities, which occur in the combustion chamber. Thermo-acoustic oscillations arise from the interactions between the acoustics of the combustion system and the heat released by the flame, as outlined by Lord Rayleigh [83]. The general implications of this feedback cycle to gas turbine combustors have been outlined in several works, see e.g. Dowling et al. [28] or Krebs et al. [53].

In the design phase of a gas turbine combustor, it is desirable to predict the thermo-acoustic stability behavior over the whole operational envelope. State of 


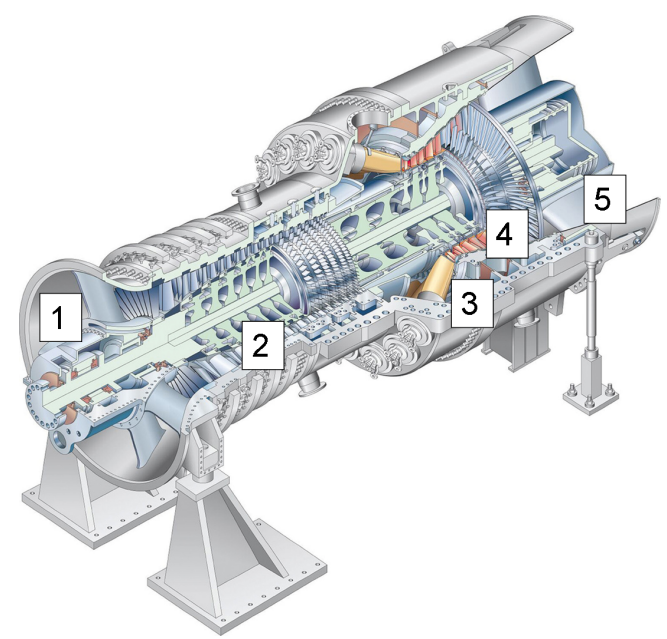

Figure 1.1: Siemens SGT5-8000H Gas turbine engine. 1 - Inlet, 2 - Compressor, 3 - Combustion chamber, 4 - Turbine, 5 - Exhaust.

the art models currently predict excited frequencies with fair accuracy. However, gas turbine manufacturers also require the knowledge of the limit cycle amplitude of the pressure oscillations, since it is this quantity which determines the operational envelope of these engines.

To investigate these limit cycle oscillations, the European Union supported the project LIMOUSINE (Limit cycles of thermo-acoustic oscillations in gas turbine combustors). This is a Marie Curie Initial Training network funded by the European Commission under Framework 7 with project number 214905. The network consists of 12 partner institutions across Europe and represents a multidisciplinary initiative to strengthen the knowledge in the field of thermo-acoustic instabilities, limit cycle behavior and the resulting mechanical vibrations and materials fatigue in combustion systems.

The work presented in this dissertation is done within the scope of the LIMOUSINE project and focuses on the numerical prediction of thermo-acoustic instabilities and limit cycle pressure oscillations. In the remainder of this chapter, the thermoacoustic instability problem will first be reviewed in more detail (section 1.2). Following that, the research goals and questions that will answered in this work will be presented (section 1.3). In section 1.4, the outline for this dissertation will be given.

\subsection{Thermo-acoustic instabilities: literature review}

The thermo-acoustic instability problem can be explained in words as follows. Inside the combustion chamber, interaction between combustion, aerodynamics and acoustics takes place. The unsteady heat release of a turbulent flame acts as an acoustic source and produces sound waves. These acoustic waves travel downstream of the combustion chamber and after reflection at the boundaries of the combustor, they return to the flame. As a result of the impinging acoustic wave, the mass flow of fresh 
reactants flowing out of the burner to the heat release zone is perturbed. Depending on the phase between pressure $\left(p^{\prime}\right)$ and heat release oscillations $\left(q^{\prime}\right)$, the flame either produces acoustic waves with an even higher amplitude or the acoustic waves are damped.

According to Rayleigh's criterion [83], see equation 1.1, thermo-acoustic instabilities are enhanced when $p^{\prime}$ and $q^{\prime}$ are in phase (the phase difference $\angle p^{\prime} q^{\prime}$ satisfies $\left.-\pi / 2<\angle p^{\prime} q^{\prime}<\pi / 2\right)$, whereas they are damped when they are out of phase $\left(\pi / 2<\angle p^{\prime} q^{\prime}<3 \pi / 2\right)$. However, a combustion system in which the unsteady heat release is adding energy to the acoustic field will not necessarily become unstable. A combustion system where $-\pi / 2<\angle p^{\prime} q^{\prime}<\pi / 2$ will only become unstable when the periodic heat release process supplies energy to the acoustic field at a rate faster than the acoustic energy is dissipated and/or transmitted through the combustors boundaries. To account for these energy losses an extended version has been derived (see e.g. Lieuwen [60], Nicoud [69] or Poinsot [75]). This extended Rayleigh criterion is given by equation 1.2, where the right hand side of the equation represents the losses.

$$
\begin{aligned}
\int_{V} \int_{0}^{\tau} p^{\prime} q^{\prime} d t d V & >0 \\
\frac{\gamma-1}{\gamma p_{0}} \int_{V} \int_{0}^{\tau} p^{\prime} q^{\prime} d t d V & >\int_{A} \int_{0}^{\tau} p^{\prime} \mathbf{u} \cdot \mathbf{n} d t d A
\end{aligned}
$$

A classic example explaining thermo-acoustic oscillations is the Rijke tube, first built by Rijke [84] in 1859. It consists of a straight duct with open ends and a heat source (e.g. a heated gauze or a flame). A sketch of a Rijke tube is presented in figure 1.2. The mode shapes of the acoustic pressure $p^{\prime}$ and acoustic velocity $u^{\prime}$ at the first natural frequency are shown in the right part of the figure. Depending on the position of the heat source, thus on the phase difference between heat release and pressure oscillations $\angle p^{\prime} Q^{\prime}$ (note that $Q^{\prime}=\int q^{\prime} d V$ ), a loud sound is produced which grows in amplitude until it is limited by nonlinear effects.
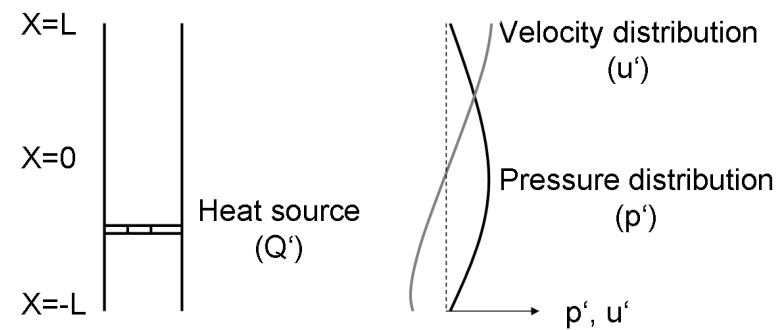

Figure 1.2: The Rijke Tube

\subsubsection{Control of thermo-acoustic instabilities}

As mentioned, the thermo-acoustic feedback loop described with the Rijke tube example is undesired in gas turbine combustors, since the large pressure amplitudes 
can damage the combustion system. Gas turbine designers have the following options for controlling thermo-acoustic instabilities:

- Helmholtz resonators. Resonators can be attached to the wall of the combustion chamber to suppress peaks in the acoustic spectrum $[9,43]$. The disadvantage of resonators is that they only work in a very narrow frequency band. Between part load and base load operation, the eigenfrequencies of the combustion system change due to the different flame temperatures, which means that resonators of different size are required to cover the entire frequency range. Another disadvantage is that for low-frequency longitudinal modes, the volume of the resonator should be quite large compared to the combustor. Often there is only little space available.* Therefore, Helmholtz resonators are mainly used for the suppression of high frequency modes.

- Active control. With active control $[27,38,66]$, a closed feedback loop is created between the pressure oscillations and the fuel mass flow. The system checks the amplitude and frequency of the instabilities and sends back a signal to the fuel mass flow controller. The phase difference between the fuel mass flow fluctuation and the pressure oscillations is chosen in such a way that the instability is damped. Due to the high costs of active control systems and the risks of damage to the engine in case the controller fails, gas turbine manufacturers are reluctant to apply this technology.

- Design changes. The thermo-acoustics of the system can be tuned by geometry changes. The length and diameter of the combustion chamber determine for instance the natural frequencies. The burner design has also an important effect. For a jet flame for instance, the length of the flame can be tuned by changing the diameter of the jet nozzle. This changes the phase (time lag) between heat release and pressure oscillations and thus changes the stability. Tuning the thermo-acoustic stability by making design changes is not always possible however, since the burner and combustion chamber have to fit in the gas turbine engine.

- Operating conditions and fuel staging. Similar to changes in the geometry, changes in operating conditions may change the natural frequencies of the combustor (due to changes in the speed of sound) or may change the phase between heat release and pressure oscillations (due to the location of the heat release zone). Many gas turbine combustion systems have multiple fuel stages. An option that is sometimes applied is firing these different fuel stages with different flame temperatures, creating an asymmetrical heat release zone and so damping the instabilities. Unfortunately, such an approach usually leads to higher $C O$ and/or $N O_{X}$ emissions.

*The eigenfrequency of a Helmholtz-resonator is given by:

$$
f=\frac{c}{2 \pi} \sqrt{\frac{A}{V \cdot L}}
$$

with $c$ the speed of sound, $V$ the volume of the resonator, $A$ the area of the necks and $L$ the neck length. 
Whatever mitigation strategy is chosen for preventing the thermo-acoustic instabilities from occurring, the thermo-acoustics of the system need to be modeled in order to assess their impact. For instance, the mode shapes of the unstable frequencies, as well as the excitation mechanism should be known. The next section discusses how these thermo-acoustic instabilities can be modeled.

\subsubsection{Modeling strategies of thermo-acoustic instabilities}

In general, there are two key strategies for studying combustion dynamics of thermoacoustic systems. The first strategy is associated with the self-excitation of acoustic modes. In this case flames themselves strongly interact with acoustic waves. When acoustic pressure fluctuations and heat release fluctuations oscillate in phase, acoustic energy is added to the system, leading to an thermo-acoustic instability. The selfexcitation of acoustic modes has both been studied experimentally (see e.g. Lieuwen et al. [63], Hield et al. [41] or Kim et al. [48]) and numerically (e.g. Chatterjee et al. [11], Kostrzewa [51] or Hernandez et al. [40]). Typical magnitudes of the dynamic pressure amplitude that has been measured are in the order of $2 \%$ to $10 \%$ of the mean pressure.

The alternative strategy to analyze the dynamics of a system is the forced response approach. In this approach, the flame is willingly excited by externally perturbing the mass flow upstream of the flame. However, this approach can only be applied to flows that do not exhibit any self-excited combustion instabilities. Utilizing an additional external forcing in the forced response study could lead to an incorrect understanding with regard to combustion dynamics being already driven by natural unstable modes.

When applying the forced response approach, a commonly used quantity is the Flame Transfer Function (FTF), which gives the heat release rate of the flame in response to acoustic fluctuations from a reference position upstream of the flame. The FTF $(F(\omega))$ is a function of frequency, and for perfectly premixed combustion systems, is often expressed as in equation 1.4:

$$
F(\omega)=\frac{\hat{Q}(\omega) / Q_{0}}{\hat{u}(\omega) / u_{0}}
$$

Here, $Q_{0}$ and $\hat{Q}$ represent the mean volumetric heat release by the flame and its fluctuation, $u_{0}$ and $\hat{u}$ represent the mean and acoustic velocity and $\omega$ the frequency.* The FTF can be obtained by experimental methods [47, 48, 62], analytical methods (such as the $n-\tau$ time lag model [16,17] or Dowling's kinematic flame model [25]), as well as from Computational Fluid Dynamics (see e.g. Armitage et al. [4], van Kampen [47] or Polifke [12,76]). In the state of the art CFD procedure, the FTF is determined by Large Eddy Simulations where the flame is excited by perturbing the boundaries of the combustor using a broadband frequency signal [12].

Flame transfer functions can be used as inputs to low order thermo-acoustic system models, which are then solved to determine the excited frequencies (via eigen-

\footnotetext{
*The following notation is used throughout this dissertation: the frequency dependent quantity $\hat{Q}(\omega)$ represents the Fourier transform of the fluctuation in the time domain $Q^{\prime}(t)$.
} 
frequencies and growth rates) of a given combustion system. These models are often one-dimensional and for gas turbine combustion chambers it is often assumed that the acoustics themselves can be considered linear. A review of available models as well as a more detailed description can be found in Chapter 2.

In this work, the thermo-acoustic instabilities are studied using numerical methods, such as Computational Fluid Dynamics. The numerical modeling approach for the self-excited and the forced response methods is schematically compared in figure 1.3. It can be seen that in the self-excited method, the whole combustion system needs to be modeled with CFD, in order to capture the acoustics of the system correctly. For a gas turbine combustion system, this is usually the part between the compressor exit and turbine inlet. In the forced response method, only the relevant parts which determine the flame dynamics are modeled (the burner and the combustion chamber). A transfer function is then determined from this CFD simulation and used as an input in a separate 1D acoustic code, which models the complete geometry.
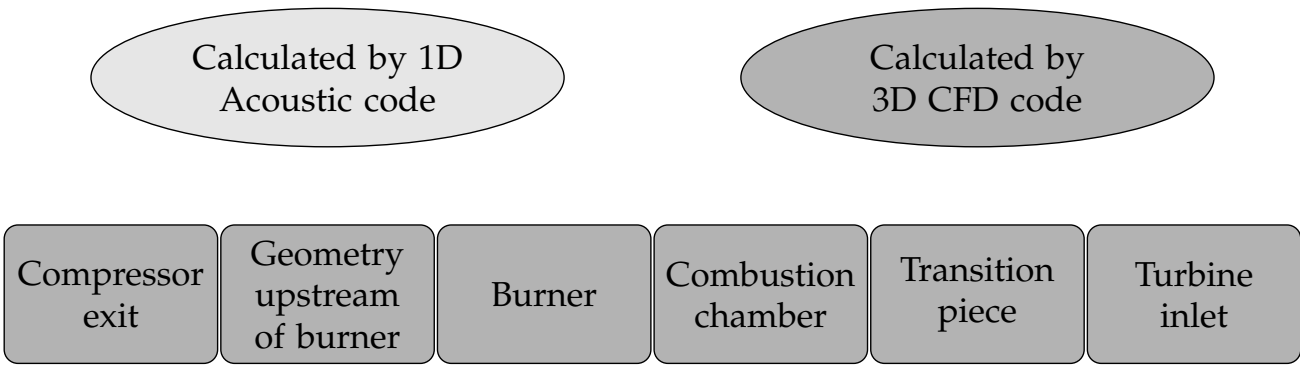

(a) Self-excited method.

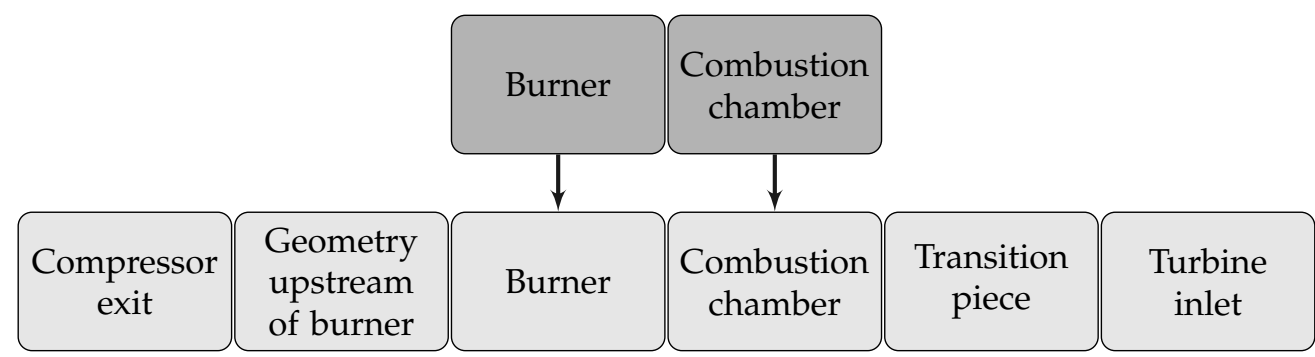

(b) Forced response method.

Figure 1.3: Comparison self-excited and forced response method.

Comparing the self-excited method and the forced response method, the following advantages can be distinguished for both methods:

\section{Forced response method:}

- A smaller CFD domain is required. This could make this method computational less expensive than the self-excited method, but this depends on the number of simulations that is required. Using the forced response method multiple 
simulations are required (a simulation without excitation and one or multiple simulations with excitation).

- Additional analysis is possible that improves the understanding of the thermoacoustics of the system. For instance, the identification of the excitation mechanisms at different frequencies and the saturation mechanism of the flame can be analyzed (in section 1.2.3 it is explained what is meant with saturation mechanism).

- Influence of design changes on the thermo-acoustic stability (e.g. an elongation of the combustion chamber) can be easily assessed, when it is assumed that this design change does not result in a different Flame Transfer Function.

- It may be possible to use smaller simulation times. Typical run-times for a simulation with the forced response method are in the order of 8 acoustic cycles (corresponding to $80 \mathrm{~ms}$ for a $100 \mathrm{~Hz}$ mode). It may be possible that it takes in the order of 50 cycles before the limit cycle pressure amplitude fully develops in a simulation using the self-excited method.

\section{Self-excited method:}

- All physics are modeled in 3D, making this method most likely more accurate than the forced response method.

- No separate 1D acoustic code is required.

- Excitation of the boundary conditions is not required.

- Only one simulation is required. For the forced response method, multiple simulations need to be performed.

In this dissertation, the main focus is on the forced response method. A comparison with the self-excited method will be provided as well in Chapter 4. As mentioned before, the work focuses on the numerical prediction of limit cycle pressure oscillations. Unfortunately, the limit cycle pressure amplitude cannot be predicted using the combination of a 1D acoustic code and the Flame Transfer Function, as the FTF is a linear model and the limit cycle pressure amplitudes are governed by nonlinear effects. This is explained in the next section. In the next section, the Flame Describing Function is introduced. The Flame Describing Function is a nonlinear extension of the Flame Transfer Function and is required for limit cycle prediction.

\subsubsection{The Flame Describing Function}

In order to predict the limit cycle pressure amplitude, nonlinear effects have to be added to the established forced response modeling approach. Currently, the heat release rate of the flame is considered the most dominant nonlinear term for gas turbine engines in power generation (Balachandran [5], Dowling [29], Lieuwen [62,63]). The nonlinear system can be explained using figure 1.4. In this sketch, the energy of a system as a function of the perturbation level, represented by the square of the 
acoustic velocity $\left|u^{\prime}\right|^{2}$, is shown. It can be seen in the figure that the amplitude of a limit cycle is governed by a balance of energy gain and losses. A limit cycle is reached when the amount of energy added to the combustion system equals the energy losses. The figure can be interpreted as a schematic representation of the extended Rayleigh criterion (equation 1.2).

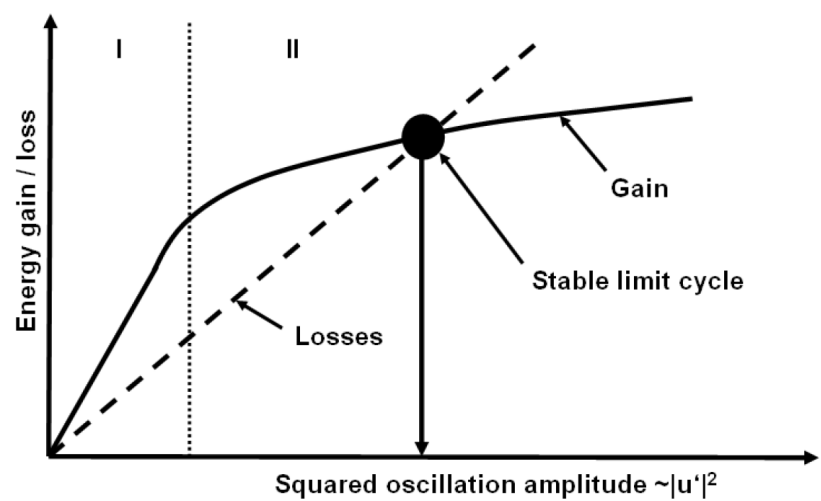

Figure 1.4: Interaction between energy losses and energy gain via unsteady heat addition. [62]

In this approach the losses present in the combustion system, such as losses through the boundaries and by turbulence (represented by the dashed line), are assumed to be linearly dependent on $\left|u^{\prime}\right|^{2}$ (as suggested by Dowling [24, 29]). This is valid when the acoustic impedance at the boundaries does not change with increasing perturbation amplitude. The flame dynamics (represented by the solid line), however, have a linear regime for smaller perturbation amplitudes (region I) as well as a nonlinear regime for larger perturbation amplitudes (region II). For linear losses and nonlinear gains, it can be seen that the acoustic energy would increase unbounded in region I since no equilibrium point can be reached. However, it is apparent that a stable limit cycle can be sustained within the nonlinear region.

One of the reasons for this nonlinear flame saturation is that the magnitude of the acoustic velocity $\left|u^{\prime}\right|$ can become of the same order of magnitude as the mean flow velocity $u_{0}[5,24,29,62,63]$. Modeling such physics requires a nonlinear model for the flame unsteadiness. The standard Flame Transfer Function given by equation 1.4 is a linear model and only describes the physics in region I of figure 1.4 (it is a function of $\omega$, but not of $\left.\left|u^{\prime}\right|\right)$. A common nonlinear modeling approach, originally introduced by Dowling [24], is to extend the FTF to the so-called Flame Describing Function $\mathcal{F}(\mathrm{FDF})$ :

$$
\mathcal{F}\left(\omega,\left|u^{\prime}\right|\right)=\frac{\hat{Q}(\omega) / Q_{0}}{\hat{u}(\omega) / u_{0}}
$$

Here $\left|u^{\prime}\right|$ stands for the root mean square acoustic velocity. It should be mentioned that the FDF corresponds to the slope of the gain in figure 1.4. With the dependency of the FDF on $\left|u^{\prime}\right|$, the decreasing slope for larger perturbation amplitudes can now be modeled. 
Currently, most work on the nonlinear heat release rate and Flame Describing Function has been experimental. Several research groups experimentally confirmed that the Flame Describing Function, and thus the heat release rate by the flame, decreases for increasing velocity amplitudes. Also it was investigated why the flame saturates for larger perturbation levels. Key references for measurements on various premixed flames include:

- Balachandran et al. $[5,6]$. In this experiment, a bluff-body ethylene flame with Reynolds-number $R e=19000$ was acoustically forced with acoustic velocities of up to $64 \%$ of the mean flow. The heat release reponse became nonlinear (the amplitude of the FDF became dependent on $\left|u^{\prime}\right|$ ) after $\left|u^{\prime}\right|$ exceeded $15 \%$ of the bulk velocity $u_{0}$. Using $\mathrm{OH}$-PLIF and $\mathrm{CH}_{2} \mathrm{O}$-PLIF images, they determined that the saturation was caused by the fact that the surface area of the flame nonlinearly evolved with larger excitation amplitudes. A smaller flame surface leads to a reduced heat release rate.

- Lieuwen et al. [61-63]. A swirl stabilized combustor with $R e=21000$ up to $R e=43000$ was excited with acoustic velocity amplitudes of up to $100 \%$ of the mean flow. They identified from their $O H$-PLIF images that due to vortexrollup and unsteady flame liftoff the area of the flame was reduced, leading to the saturation at higher amplitudes.

- Palies et al. [71, 72]. In this experiment, a swirl flame was studied as well, with up to $72 \%$ excitation. Also here it was observed that the flame surface increased nonlinearly with excitation amplitude, which is consistent with the observations of Balachandran et al. [5] and Lieuwen et al. [61].

- Schimek et al. [86]. A swirl stabilized combustor with $R e=35000$ was excited with acoustic velocity amplitudes of up to $70 \%$ of the mean flow. Schimek et al. determined from their $\mathrm{OH}^{*}$-chemiluminescence images that the main mechanism leading to saturation was flame quenching due to local extinction and re-ignition. It could also be observed from the $O H^{*}$ images that the flame angle significantly fluctuated. Although this is not disucssed in Ref [86], it could be that this fluctuating flame angle modulates the flame surface in a nonlinear way.

Importantly, these authors also found that during self-excited measurements that the acoustic limit cycle pressure amplitudes were an order of magnitude smaller than the mean pressure. For example, Lieuwen et al. [63] measured pressure amplitudes of up to $2 \%$ of the mean pressure, thus justifying the treatment of the acoustics themselves as linear.

Although different research groups experimentally determined Flame Describing Functions, the work on the numerical prediction of FDFs from CFD is very limited. One of the only numerical predictions of the Flame Describing Function has been done by Armitage et al. [3]. Armitage et al. investigated the FDF for the bluff body flame of Balachandran [5], in a two dimensional domain using a URANS approach. They were correctly able to reproduce the saturation of the flame that was found in 
the measurements. The flame was close to laminar however. For highly turbulent flows no such investigation has, to the knowledge of the author, been performed so far.

\subsubsection{Perfectly versus partially premixed flames}

Up to now, only perfectly premixed combustion systems have been considered. In perfectly premixed combustion systems (see figure 1.5(a)), fuel and air are mixed far upstream of the burner. This means that the equivalence ratio $\Phi$ does not fluctuate due to thermo-acoustic instabilities.

However, for safety reasons, perfectly premixed combustion is not applied in industrial combustion systems. Industrial combustion systems operate in the partially premixed operating mode (see figure 1.5(b)). Air and fuel are mixed in a mixing passage shortly upstream of the flame.

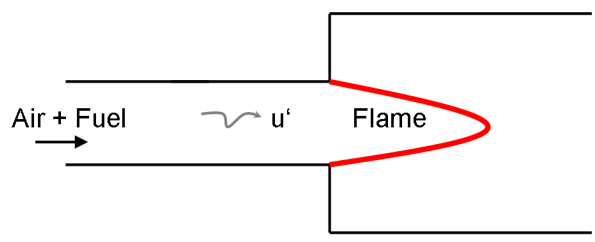

(a) Perfectly premixed flame.

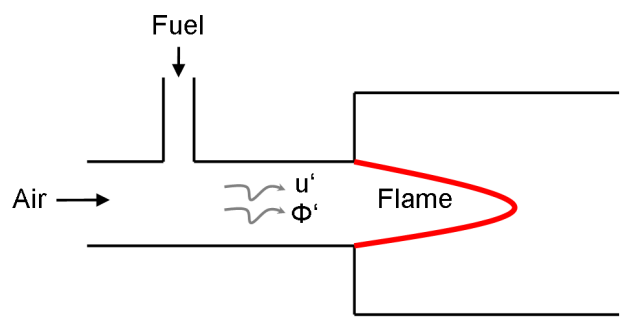

(b) Partially premixed flame.

Figure 1.5: Comparison of perfectly and partially premixed combustion systems.

In partially premixed operation, a fluctuation of air velocity automatically induces a fluctuation in equivalence ratio. Often the fuel enters the premixing passage through the fuel nozzles with high Mach number. In this case the fuel mass flow does not significantly fluctuate. In the limiting case of choked flow $(M a=1)$ the fuel mass flow does not change at all. When the fuel mass flow is approximately constant, fluctuations of air velocity and equivalence ratio are coupled.

The Flame Describing Function of equation 1.5 only takes into account velocity fluctuations. It is a so-called Single Input, Single Output (SISO) model. Principally it could be used for partially premixed combustion systems as well, since due to the coupling between velocity and equivalence ratio, the influence of equivalence ratio fluctuations is implicitly included in the Flame Describing Function through the heat release response.

However, for partially premixed systems, Multiple Input, Single Output (MISO) models have been derived as well (see e.g. Huber [44]). Compared to SISO models, these kind of models allow for studying the influence of equivalence ratio and air fluctuations separately. Depending on the phase difference between air, equivalence ratio and heat release fluctuations, it may be possible that one type of fluctuation has a stabilizing effect, while the other one a destabilizing. Although such a scenario would be implicitly captured in SISO models, which kind of fluctuation is the one that actually drives the instability can only be identified with the MISO approach. 
Therefore a main advantage of MISO models is to improve the understanding of a given combustion system. For gas turbine manufacturers, another practical benefit is that the influence of certain design changes on the thermo-acoustic stability (e.g. the position of the fuel injection) can easily be assessed.

Huber [44] derived the following linear Flame Transfer Function MISO model. The model starts by considering the global heat release of the combustion process, which is given by:

$$
Q=m_{f u e l} H_{f}=f_{T} \rho_{u} \dot{V} H_{f}
$$

with $m_{f u e l}$ the fuel mass flow rate, $H_{f}$ (unit J/kg fuel) the specific enthalpy of reaction, $\rho_{u}$ the density of the unburnt mixture, $f_{T}$ the fuel mixture fraction and $\dot{V}$ the volume flow. The total differential of the heat release yields:

$$
d Q=\left(\frac{\partial Q}{\partial f_{T}}\right) d f_{T}+\left(\frac{\partial Q}{\partial \rho_{u}}\right) d \rho_{u}+\left(\frac{\partial Q}{\partial \dot{V}}\right) d \dot{V}
$$

The heat release rate fluctuations can then be decomposed into separate contributions of fluctuating unburnt density, velocity and mixture fraction:

$$
\frac{Q^{\prime}}{Q_{0}} \cong \frac{f_{T}^{\prime}}{f_{T 0}}+\frac{\rho_{u}^{\prime}}{\rho_{u 0}}+\frac{\dot{V}^{\prime}}{\dot{V}_{0}}
$$

The fluctuations of the unburnt density in equation 1.8 can usually be neglected in typical lean premixed combustion systems burning hydrocarbon fuels at low Mach numbers (see Huber [44]). By making use of the fact that $\dot{V}=A \cdot u$, the relation $f_{T}^{\prime} / f_{T 0}=\Phi^{\prime} / \Phi_{0}$ and introducing separate Flame Transfer Functions for velocity fluctuations $\left(F_{u}\right)$ and equivalence ratio fluctuations $\left(F_{\Phi}\right)$, Huber [44] derived the following MISO model:

$$
\frac{\hat{Q}(\omega)}{Q_{0}}=F_{u}(\omega) \frac{\hat{u}(\omega)}{u_{0}}+F_{\Phi}(\omega) \frac{\hat{\Phi}(\omega)}{\Phi_{0}}
$$

Huber developed a method to simultaneously identify the Flame Transfer Functions $F_{u}$ and $F_{\Phi}$ from a transient CFD simulation. In simulations, Huber excited both the air and fuel inlets with uncorrelated broadband signals. An important conclusion from his work was that a unique set of transfer functions can only be found in case both inlets are perturbed.

In the scope of this work, it will be investigated if an extension of Huber's work to MISO Flame Describing Functions is possible. The following model will be investigated:

$$
\frac{\hat{Q}(\omega)}{Q_{0}}=\mathcal{F}_{u}\left(\omega,\left|u^{\prime}\right|\right) \frac{\hat{u}(\omega)}{u_{0}}+\mathcal{F}_{\Phi}\left(\omega,\left|\Phi^{\prime}\right|\right) \frac{\hat{\Phi}(\omega)}{\Phi_{0}}
$$

It should be noticed that in the derivation of equation 1.9, it has been assumed that the fluctuations are small and linear. Therefore the model does not contain cross 
terms between equivalence ratio fluctuations and velocity fluctuations; both contributions can be linearly superimposed. Obviously this does not have to be the case during limit cycle oscillations. It should therefore be mentioned that a model such as 1.10 may introduce some error, because it does not take into account cross terms between equivalence ratio and velocity fluctuations.

\subsection{Research questions}

The goal of the work presented in this dissertation is to develop a method that can be used to numerically predict the limit cycle pressure amplitude. The method is an extension of the current forced response method. Both perfectly premixed and partially premixed flames will be investigated. Also it is investigated why the flame saturates for large perturbation levels. The method will first be applied to generic lab scale test rigs, but it will also be determined if the method can be applied to industrial combustion systems, which feature additional challenges compared to generic lab scale configurations.

In order to structure the research presented in this dissertation, the following research questions were posed:

1. Can the limit cycle pressure amplitude be predicted using a one-dimensional stability model?

(a) What are the requirements for an accurate prediction of the limit cycle pressure amplitude?

(b) What is the sensitivity of the prediction of the limit cycle pressure amplitude on the Flame Describing Function and the boundary conditions?

(c) What are the strengths and limitations of such models?

2. Can the Flame Describing Function be predicted using CFD (LES)?

(a) What are the requirements of the CFD code for an accurate prediction of the Flame Describing Function?

(b) What are the strengths and limitations of the approach?

3. What is the mechanism leading to saturation of the flame?

4. Can the proposed methodology (combination of identification of the Flame Describing Function using LES and a one-dimensional stability tool) be applied to an industrial combustion system to predict limit cycles?

(a) What are the different aspects in the approach and difficulties encountered compared to a generic lab scale combustor?

(b) Can a multiple-input FDF be determined, for a partially premixed system with both equivalence ratio and velocity fluctuations?

5. What is the performance of the forced response method compared to the selfexcited method? 


\subsection{Outline}

In Chapter 2 the one-dimensional thermo-acoustic stability tool, that was developed as part of this work, will be described and validated with test cases from literature, for which data on the Flame Describing Function and measurements of the limit cycle pressure amplitudes were available. Data required for answering Research question 1 will be collected as well.

In Chapter 3 the CFD code used in this work is explained, as well as the procedure to determine the Flame Describing Function from numerical simulations. Special focus is on the specific requirements for obtaining the Flame Describing Functions (boundary conditions, sampling of data and postprocessing).

In Chapter 4 first the Flame Describing Function of a swirl stabilized flame, for which measurement data were provided by the University of Berlin (Schimek et al. [86]), is determined using CFD and the saturation mechanism of this flame is studied. The resulting Flame Describing Function is then used to predict the limit cycle pressure amplitude. Furthermore, a comparison of the forced response versus self-excited CFD simulations is made. In this chapter, data for Research questions 2, 3 and 5 is collected.

In Chapter 5 the combined methodology (combination of identification of the Flame Describing Function using LES and a one-dimensional stability tool) is applied to an partially premixed industrial combustion system. In this chapter, Research question 4 is addressed.

In Chapter 6 conclusions regarding the research questions are provided and recommendations for future work are given. 


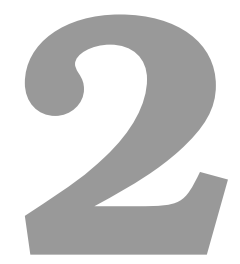

\section{Thermo-acoustic instability model}

\subsection{Introduction}

In this work, the limit cycle pressure amplitude is predicted using a combination of the Flame Describing Function, discussed in the previous chapter, and a nonlinear thermo-acoustic instability model. This nonlinear model has been developed within this work as an extension to an existing linear stability model (used for prediction of eigenfrequencies and growth rates).

The main objective of this chapter is to validate the nonlinear model with measurement data from literature. Also, in order to answer Research question 1 (see section 1.3), a parametric study to assess the strengths and weaknesses of such models is performed.

The chapter is structured as follows: section 2.1.1 provides an overview of existing types of linear instability models and it is motivated why a specific type of instability model has been chosen for the extension to limit cycle prediction. Next, in section 2.2, the model formulation and implementation are discussed in detail. The validation of the code, as well as the parametric study, are the subjects of section 2.3.

\subsubsection{Overview of existing models}

First, an overview of existing linear thermo-acoustic instability models is given. Linear models usually model the flame-acoustics interaction with a Flame Transfer Function and can be used to determine the excited frequencies and growth rates of a given combustion system. In the past, several stability models have been developed by different research groups. Two common types are network models, which work in the frequency domain, and time dependent models. For both types of models, both 1D and 3D variants have been developed. 


\section{D Network models (frequency domain)}

In this approach, the complete combustion system is modeled as a network of 1D acoustic elements. Each element represents a specific component of the system (such as a duct, area change or flame) and each element has constant mean flow properties (e.g. density, temperature and velocity). The propagation of the acoustic pressure and acoustic velocity through these elements is usually modeled using transfer matrices, which are derived from the conservation equations. The flame can be assumed to be infinitely thin or a one-dimensional heat release distribution can be prescribed.

Network models solve for the complex eigenfrequencies $\left(\omega=\omega_{r}+i \cdot \omega_{i}\right)$ of the system, where the real part $\omega_{r}$ is the frequency and the imaginary part $\omega_{i}$ corresponds to the growth rate. Key references for 1D Network models include Hubbard et al. [42], Kim et al. [48], Krüger et al. [57], Polifke et al. [77] and Schuermans et al. [89]. In Appendix A the Transfer Matrix Approach, which is an example of a 1D frequency domain network model, is discussed and more information about this type of models is provided.

\section{D time dependent models}

For time dependent models, a typical solution methodology is the modified Galerkin approach, as described by Culick et al. [18-21]. Originally the approach was developed to study combustion instability in rocket engines, but later the modified Galerkin approach has been applied to characterize the thermo-acoustic excitation of gas turbine combustion systems as well (see for instance Dowling et al. [28], Krebs et al. [54] or Sujith et al. [7]).

In the modified Galerkin approach, it is assumed that the thermo-acoustic problem can be decoupled in two steps. The first step consists of solving the spatial problem, in which the acoustic mode shapes are determined. These are calculated from a homogeneous Helmholtz equation, which means the mode shapes are not influenced by source terms (e.g. from combustion). The second step consists of perturbing these acoustic modes in the time domain by activating all mechanisms that can damp or excite a given mode. This leads to a temporal solution oscillating near the selected frequency, which exhibits temporal growth or decay. It should be noted that the frequency of the mode under investigation can shift due to these damping or excitation mechanisms, but the shape of the acoustic modes will not change.

\section{D frequency domain models}

This approach is based on a Finite Element discretization of the inhomogeneous Helmholtz equation on a 3D mesh. The mean flow velocity, temperature and heat release fields are usually taken from a CFD simulation and mapped on this mesh. This method solves for the 3D eigenmodes and growth rates, which can be axial modes, tangential modes, radial modes or combinations. Although the method is expected to be more accurate than $1 \mathrm{D}$ network models, the computational requirements are much higher. Key references include Nicoud et al. [70] and Selle et al. [91]. 


\section{D time dependent models}

In 3D time dependent approaches, the inhomogeneous wave equation or Euler equations can either be directly solved in the time domain (e.g. Pankiewitz et al. [74]) or using the Galerkin method (see for instance Arens [2]). Similar to the 3D frequency domain models, any type of mode shape (axial, tangential, radial) can be resolved. It has the disadvantage that complex valued impedance boundary conditions are difficult to handle, but the advantage that it can easily be extended to predict limit cycles.

\subsubsection{Extension to a nonlinear instability model}

Few attempts have been made so far to develop a nonlinear instability model for limit cycle prediction based on the Flame Describing Function. Recently, Palies et al. [72] introduced a 1D frequency domain model that solves for real valued eigenfrequencies $\omega=\omega_{r}\left(\left|u^{\prime}\right|\right)$. They determined the velocity amplitude $\left|u^{\prime}\right|$ such that the complex part of the eigenfrequency, $\omega_{i}$, becomes zero. On the other hand, Selimefendigil and Polifke [90] applied a Galerkin type model to predict the limit cycle pressure amplitude in a Rijke tube. Their model belongs to the class of $1 \mathrm{D}$ time dependent models.

In the scope of this work, a 1D approach was chosen for the nonlinear extension to limit cycle prediction, since these methods are much cheaper than the 3D approaches. Also most modes of interest are of one-dimensional nature. Comparing the 1D time and frequency domain approaches, models that work in the frequency domain have the advantage that the acoustic mode shapes can be predicted more accurately (since no decoupling between a spatial and a temporal problem is made) and that complex valued, frequency dependent boundary conditions can easily be implemented. On the other hand, time domain models, such as the modified Galerkin approach, have better capabilities of predicting time dependent phenomena than the frequency domain models. Nonlinear terms such as the Flame Describing Function are time dependent, since they change as the acoustic velocity changes. This makes it practical to implement the FDF in these kind of models. Given this advantage, within this work, nonlinear effects were implemented into a temporal based system model.

The model is an extension of the Generalized Instability Model (GIM) $[81,82,92]$ and will be discussed in detail as well as validated in the remaining part of this chapter. For reference, in Appendix A, a linear 1D frequency domain network model is discussed as well and in this appendix it is also described how nonlinear effect may be implemented using such kinds of models.

\subsection{Model formulation}

In this section, the equations that are solved by the Generalized Instability Model are derived. First in subsections 2.2.1 and 2.2.2, the governing equations of the linear modified Galerkin Approach are given. In this work, additional terms required for limit cycle prediction, namely the Flame Describing Function and the losses through 
the boundaries, have been implemented in the code. The implementation of these terms is discussed in subsections 2.2.3 and 2.2.4. In subsection 2.2.5, the solution procedure is discussed.

It should be noted that the Flame Describing Function is assumed to be the only nonlinear term in the model. The acoustic fluctuations themselves are assumed to be linear, which is valid for acoustic pressures that are small compared to the mean pressure (see for instance Lieuwen et al. [63]).

\subsubsection{Governing equations}

The model starts from the Euler equations for a non-heat conducting, inviscid fluid with heat release due to chemical reactions:

Mass: *

$$
\frac{\partial \rho}{\partial t}+\nabla \cdot(\rho \mathbf{u})=0
$$

Momentum:

$$
\rho \frac{\partial \mathbf{u}}{\partial t}+\rho(\mathbf{u} \cdot \nabla) \mathbf{u}=-\nabla p
$$

Internal energy:

$$
\frac{\partial(\rho e)}{\partial t}+\nabla \cdot(\rho \mathbf{u} e)=-p \nabla \cdot \mathbf{u}+q
$$

Here $\rho$ is the density, $\mathbf{u}$ the velocity, $p$ the pressure, $T$ the temperature, $e$ the internal energy and $q$ is the heat release density (in $J / \mathrm{m}^{3} / \mathrm{s}$ ). Assuming a calorically perfect gas, the expressions $p=\rho R T$ and $e=C_{V} T=\frac{1}{\gamma-1} p / \rho$ can be substituted. If secondly the variables are expanded in terms of their mean and fluctuating parts, e.g. $p=p_{0}+p^{\prime}$, and they are introduced into equations 2.1 to 2.3, then, after linearizing with respect to the perturbation quantities, the following equation set is found:

$$
\begin{aligned}
\rho_{0} \frac{\partial \mathbf{u}^{\prime}}{\partial t}+\nabla p^{\prime} & =-\rho_{0}\left(\mathbf{u}_{\mathbf{0}} \cdot \nabla\right) \mathbf{u}^{\prime}-\rho_{0}\left(\mathbf{u}^{\prime} \cdot \nabla\right) \mathbf{u}_{\mathbf{0}} \\
\frac{\partial p^{\prime}}{\partial t}+\gamma p_{0} \nabla \cdot \mathbf{u}^{\prime} & =-\mathbf{u}_{\mathbf{0}} \cdot \nabla p^{\prime}-\gamma p^{\prime} \nabla \cdot \mathbf{u}_{\mathbf{0}}+(\gamma-1) q^{\prime}
\end{aligned}
$$

Here terms that are second order in the fluctuating quantities as well as gradients in the mean pressure and density have been neglected. Taking the gradient of equation scalar:

*In this dissertation, a bold printed quantity (e.g. u) represents a vector, whereas $u_{x}$ represents a

$$
\nabla \cdot(\rho \mathbf{u})=\frac{\partial\left(\rho u_{x}\right)}{\partial x}+\frac{\partial\left(\rho u_{y}\right)}{\partial y}+\frac{\partial\left(\rho u_{z}\right)}{\partial z}
$$


2.4, differentiating equation 2.5 with respect to time, and adding the results leads to an inhomogeneous wave equation for the acoustic pressure:

$$
\nabla^{2} p^{\prime}-\frac{1}{c^{2}} \frac{\partial^{2} p^{\prime}}{\partial t^{2}}=h
$$

with $c$ the speed of sound and $h$ :

$$
h=-\rho_{0} \nabla\left[\left(\mathbf{u}_{\mathbf{0}} \cdot \nabla\right) \mathbf{u}^{\prime}+\left(\mathbf{u}^{\prime} \cdot \nabla\right) \mathbf{u}_{\mathbf{0}}\right]+\frac{1}{c^{2}}\left[\gamma \nabla \cdot \mathbf{u}_{\mathbf{0}}+\mathbf{u}_{\mathbf{0}} \cdot \nabla\right] \frac{\partial p^{\prime}}{\partial t}-\frac{\gamma-1}{c^{2}} \frac{\partial q^{\prime}}{\partial t}
$$

This result is similar to the results of Culick et al. [18-21] or Portillo et al. [81, 82,92].

\subsubsection{Expansion as Galerkin series}

In the modified Galerkin approach [18-21,54], it is assumed that the pressure and velocity fluctuations can be written as:

$$
\begin{aligned}
p^{\prime}(\mathbf{x}, t) & =p_{0} \sum_{n=1}^{N} \Psi_{n}(\mathbf{x}) \eta_{n}(t) \\
\mathbf{u}^{\prime}(\mathbf{x}, t) & =\sum_{n=1}^{N} \frac{1}{\gamma k_{n}^{2}} \nabla \Psi_{n}(\mathbf{x}) \dot{\eta}_{n}(t)
\end{aligned}
$$

The Galerkin approach decouples the wave equation into two ordinary differential equations, by introducing the trial functions $\Psi_{n}(\mathbf{x})$, which are only dependent on the spatial coordinate, and $\eta_{n}(t)$ which is only dependent on time. The functions $\Psi_{n}(\mathbf{x})$ are chosen to be equal to the acoustic eigenmodes and the total solution can be written as the sum over all modes. The expression for the acoustic velocity (equation 2.9) follows directly from the continuity equation (assuming zero mean flow).

The modes $\Psi_{n}(\mathbf{x})$ are calculated from the homogeneous Helmholtz equation, which is obtained by inserting equation 2.8 into equation 2.6 and setting $h=0$. The Helmholtz equation is:

$$
\nabla^{2} \Psi_{n}+k_{n}^{2} \Psi_{n}=0
$$

with $k_{n}$ is the acoustic wave number.

In this approach the right hand side of equation 2.6 does not have an influence on the mode shapes $\Psi_{n}$. The role of the inhomogeneous source term $h$ is then to provide a frequency shift to the modes and to damp or excite them. After the mode shapes have been calculated, the time evolution $\eta(t)$ can be calculated. The following differ- 
ential equation is solved for the temporal evolution of the $n$-th mode:

$$
\begin{aligned}
\ddot{\eta}_{n}+\omega_{n}^{2} \eta_{n} & =-\frac{1}{p_{0} \int_{V} \Psi_{n}^{2} d V} F_{n}(\eta, \dot{\eta}, \ddot{\eta}) \\
F_{n}(\eta, \dot{\eta}, \ddot{\eta}) & =\underbrace{-\int_{V} c^{2} h \Psi_{n} d V}_{\text {Mean flow \& heat release (eq. 2.7) }}+\underbrace{\oint_{\partial V} c^{2}\left[\Psi_{n} f_{0}+f_{1} \nabla \Psi_{n}\right] \cdot \mathbf{n} d A}_{\text {Boundary conditions }}
\end{aligned}
$$

Here the forcing term $F_{n}$ can be fully evaluated in terms of $\eta$ by inserting equations 2.7 to 2.9. The first term of $F_{n}$ represents forcing or damping by the inhomogeneous source term of the wave equation $h$ (equation 2.7) and the second term represents the acoustic energy flow through the boundaries, where $f_{0}=-\nabla p^{\prime}$ and $f_{1}=p^{\prime}$ at the boundaries. Because the derivation of equation 2.11 and 2.12 is lengthy, it is given in Appendix B.

\subsubsection{Treatment of boundary conditions}

The boundary conditions used to solve equations 2.10 and 2.11 can be arbitrary, depending on the geometry. Usually acoustically open boundaries $\left(p^{\prime}=0\right.$ or $\left.\Psi_{n}=0\right)$ or closed boundaries $\left(u^{\prime}=0\right.$ or $\left.\nabla \Psi_{n}=0\right)$ are used. Boundary conditions in the mixed form $Z=p^{\prime} / u^{\prime}$ (impedance boundary condition) have also been implemented into the nonlinear version of the Generalized Instability Model. These boundary conditions are very important for limit cycle prediction, since they provide the required loss mechanism sketched in figure 1.4 of Chapter 1.

A useful quantity when dealing with impedance boundary conditions is the reflection coefficient $R$, which represents the ratio between the amplitudes of the upstream and downstream traveling waves. It can be related to the impedance $Z(\omega)$ as:

$$
R=\frac{Z(\omega)-\rho_{0} c}{Z(\omega)+\rho_{0} c}=\frac{\hat{p} / \hat{u}-\rho_{0} c}{\hat{p} / \hat{u}+\rho_{0} c}
$$

The reflection coefficient $R$ can take values between -1 and 1 . Written in the frequency domain, it may be a complex valued property. Written in the time domain, the reflection coefficient can have a magnitude $|R|$ and a phase $\angle R$. A real valued reflection coefficient of $R=-1$ represents an open boundary condition $\left(p^{\prime}=0\right)$ and is fully reflecting. In this case, the term $\left[\Psi_{n} f_{0}+f_{1} \nabla \Psi_{n}\right]$ of equation 2.12 vanishes, since $f_{1}=0$ and $\Psi_{n}=0$ at the boundary. This means that the term does not contribute to $F_{n}$ (does not provide any damping), as expected. The same is true for $R=1$, which represents a closed boundary condition $\left(u^{\prime}=0\right)$. In this case it follows that $f_{0}=0$ and $\nabla \Psi_{n}=0$, so all boundary condition terms in equation 2.12 vanish as well.

In case a non-fully reflecting boundary condition is used $(-1<R<1)$, an impedance formulation is required. For the implementation of the impedance boundary conditions, the reflection coefficient and the impedance are expressed in the time domain and the following substitutions are made: 


$$
\begin{array}{rlrl}
f_{0}=-\nabla p^{\prime}=\rho_{0} \frac{\partial u^{\prime}}{\partial t} & =\rho_{0}\left|\frac{1}{Z(\omega)}\right| \frac{\partial p^{\prime}}{\partial t}\left(t-\frac{\angle(1 / Z(\omega))}{\omega}\right) \\
f_{1}=p^{\prime} & =\quad & |Z(\omega)| u^{\prime}\left(t-\frac{\angle(Z(\omega))}{\omega}\right)
\end{array}
$$

Here equation 2.14 follows directly from the momentum equation (neglecting mean flow). It can be seen that there can be a time lag between the velocity and pressure fluctuations, to account for the fact that the impedance may be a complex valued quantity. This is expressed by the term $\angle(Z(\omega)) / \omega$. It should also be noticed that when the reflection coefficient is close to $R=1, \nabla \Psi_{n} \approx 0$, and in this case, the term $f_{1} \nabla \Psi_{n}$ is negligible small compared to $\Psi_{n} f_{0}$. On the other hand if the reflection coefficient is close to $R=-1, \Psi_{n} \approx 0$ and the term $\Psi_{n} f_{0}$ is negligible compared to $f_{1} \nabla \Psi_{n}$.

\subsubsection{Treatment of heat release rate}

As discussed in section 1.2.3, nonlinear heat release models are required for limit cycle prediction. Therefore the Flame Describing Function, given by equation 1.5 has been implemented in the code. In the time domain, equation 1.5 can be rewritten as:

$$
\begin{aligned}
\frac{Q^{\prime}(t)}{Q_{0}} & =\left|\mathcal{F}\left(\omega,\left|u^{\prime}\right|\right)\right| \frac{u^{\prime}(t-\tau)}{u_{0}} \\
\tau & =\frac{\angle \mathcal{F}\left(\omega,\left|u^{\prime}\right|\right)}{\omega}
\end{aligned}
$$

The term $\left|\mathcal{F}\left(\omega,\left|u^{\prime}\right|\right)\right|$ represents the gain, as a function of frequency and root mean square of the acoustic velocity. The acoustic velocity is evaluated on a reference plane upstream of the flame.

Written in the time domain, the heat release model contains a time lag, which accounts for the fact that a fluctuation in the heat release lags a perturbation of the velocity by a certain time $\tau$. Compared to a classical $n-\tau$ model $Q^{\prime}(t) \propto n \cdot u^{\prime}(t-$ $\tau)[16,17]$, where both the gain $n$ and time lag $\tau$ are assumed to be constant, the formulation of equation 2.16 takes into account the dependency of the gain and time lag on $\left|u^{\prime}\right|$ and thus describes the nonlinear response of the flame.

Noting that $Q=\int q d V$, equation 2.16 can be substituted into equation 2.7 and 2.12 , which results in a closed differential equation for the temporal evolution of $\eta$.

\subsubsection{Solution procedure}

The Generalized Instability Model can solve the equations given in section 2.2.1 to 2.2.4 in a network of cylindrical tubes. For each of these elements, the length, diameter and mean flow properties (pressure, velocity, temperature, density, speed of sound) are specified. These mean properties can be discontinuous over the different elements. 
Based on the geometry, mean flow properties and boundary conditions that are specified, the first step is calculating the acoustic modes $\Psi_{n}$ from equation 2.10. This equation is solved with the linear Euler equations solver (LEE), which was developed by Yu et al. [95].

After the calculation of the mode shapes, all mechanisms that can damp or excite the acoustic modes are activated (see equation and 2.7 and 2.12). These are required for the temporal evolution $\eta_{n}$ of each acoustic mode.

The final ordinary differential equation that is solved has the following form:

$$
f\left(\eta, \dot{\eta}, \ddot{\eta}, \dot{\eta}\left(t-\tau_{i}\right), \ddot{\eta}\left(t-\tau_{i}\right)\right)=0
$$

Equation 2.18 may contain multiple terms that contain a time delay, namely the boundary conditions and the heat release model. This is expressed by the subscript " $i$ ". The equation has been solved with Matlab's built-in solver for delay differential equations: the DDE23-solver [65].

The Flame Describing Function $\mathcal{F}\left(\omega,\left|u^{\prime}\right|\right)$ is specified as a look-up table. During the time integration of equation 2.18 , the root mean square velocity at the desired reference location is continuously updated based on $\Psi$ and $\eta$. The rms-velocity at time $t$ is determined based on the instantaneous acoustic velocity in the interval $[t-T, t]$, where $T=1 / f$ corresponds to one period of oscillation. The Flame Describing Function $\mathcal{F}\left(\omega,\left|u^{\prime}\right|\right)$ is then interpolated based on the new rms-velocity and the forcing term $F_{n}$ is updated and used for the next time step. The procedure continues until a limit cycle has been reached.

In figure 2.1, a flowchart showing the solution algorithm described above is provided.

\subsection{Validation}

In this section, the nonlinear model is validated with experimental data found in literature. The section also aims to clarify the solution procedure that has been described above.

\subsubsection{Bluff body stabilized flame}

The validation test case selected corresponds to the bluff body stabilized burner, for which self-excited combustion dynamics were measured by Hield et al. [41]. The burner consists of a 1 meter long pipe section with constant cross-section, with a flame holder in the middle used to stabilize the flame. The fuel was propane, the inlet boundary condition was choked, and the outlet boundary condition could be either open or choked. A sketch of the rig is shown in figure 2.2.

The heat release of the flame was captured with $\mathrm{CH}^{*}$-chemiluminescence and the acoustic pressure was recorded upstream and downstream of the flame holder. They calculated an acoustic velocity based on their pressure measurements and combined them with the $C H^{*}$ measurements to obtain a Flame Describing Function. 


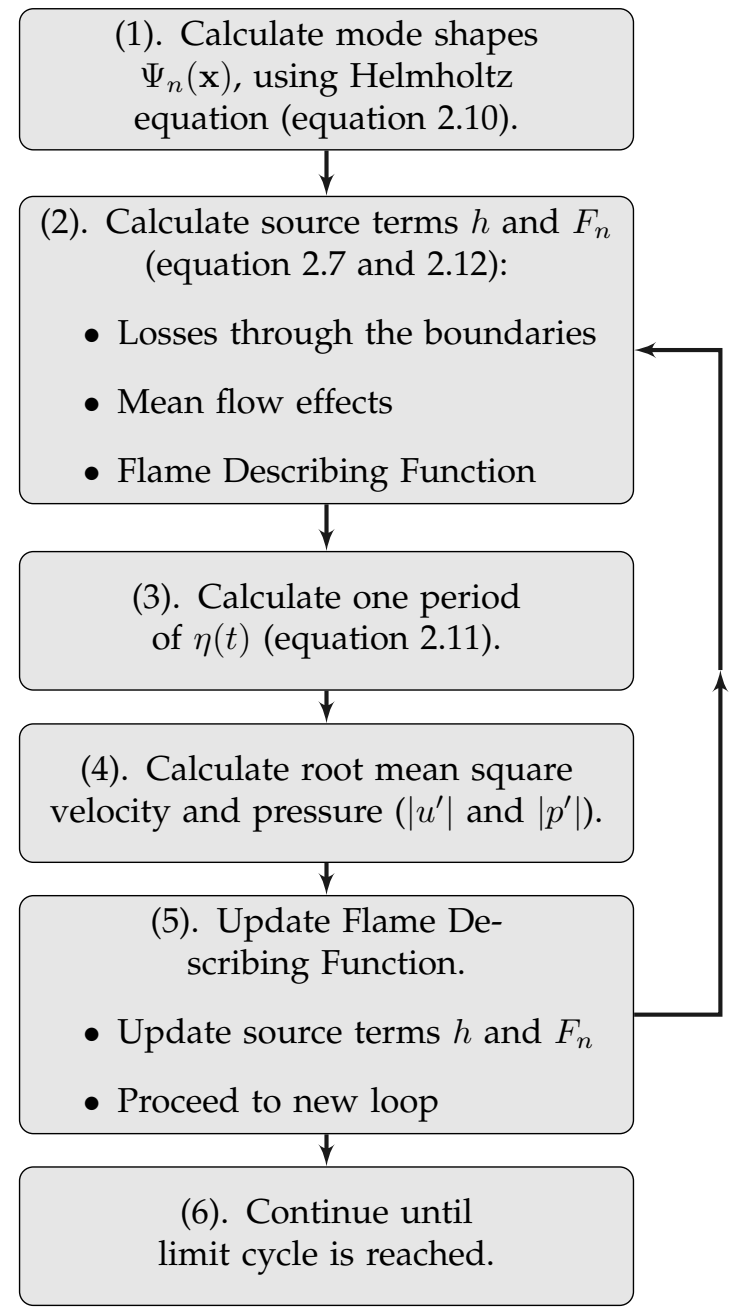

Figure 2.1: GIM solution algorithm.

Hield et al. investigated different operating points, the following have been modeled in GIM:

- Inlet Mach number $M a=0.040$, Equivalence ratio $\Phi=1$ and open outlet boundary condition.

- Inlet Mach number $M a=0.034$, Equivalence ratio $\Phi=0.78$ and open outlet boundary condition.

The GIM model, as indicated in figure 2.2, consists of 3 domains: the section upstream of the flame holder, the flame holder and the section downstream of the flame holder. Combustion takes place shortly downstream of the flame holder. Each section was assumed to have constant mean flow properties which are tabulated 


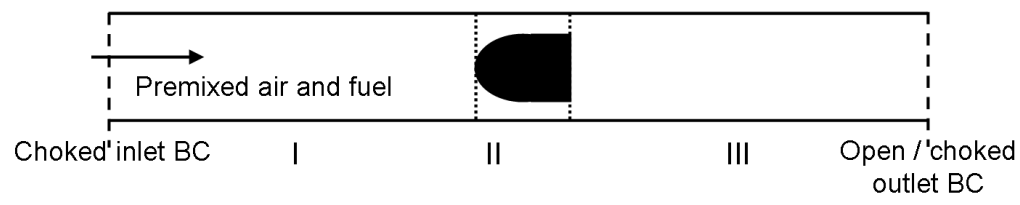

Figure 2.2: Schematic drawing of the bluff body stabilized burner.

in table 2.1. The temperature in Domain III was assumed to be the adiabatic flame temperature. These data have either been taken from Ref [41] or have been calculated based on the available data.

\begin{tabular}{lccccccc}
\hline Op. point & Domain & $\mathbf{L}[\mathbf{m}]$ & $\mathbf{A}\left[\mathbf{m}^{\mathbf{2}}\right]$ & $\mathbf{u}[\mathbf{m} / \mathbf{s}]$ & $\mathbf{c}[\mathbf{m} / \mathbf{s}]$ & $\mathbf{p}[\mathbf{b a r}]$ & $\mathbf{T}[\mathbf{K}]$ \\
\hline$M a=0.04$, & I & 0.44 & 0.00196 & 13.5 & 336 & 1 & 293 \\
$\Phi=1$ & II & 0.06 & 0.00057 & 46.1 & 336 & 1 & 293 \\
& III & 0.50 & 0.00196 & 109 & 915 & 1 & 2264 \\
\hline$M a=0.034$, & I & 0.44 & 0.00196 & 11.4 & 336 & 1 & 293 \\
$\Phi=0.78$ & II & 0.06 & 0.00057 & 39.2 & 336 & 1 & 293 \\
& III & 0.50 & 0.00196 & 82.3 & 862 & 1 & 2008 \\
\hline
\end{tabular}

Table 2.1: Mean flow properties used for the different domains and operating points.

\section{Mode shape calculation}

Using the input parameters of table 2.1, first the acoustic mode shapes have been calculated by solving the homogeneous Helmholtz equation (equation 2.10). The acoustic mode shapes, over one period of oscillation, for the first three eigenmodes of the operating point with $\Phi=1$ are plotted in figure 2.3. It can be seen that the mode shapes are discontinuous at the location of the bluff body $(x=0.44 \mathrm{~m})$, which can be explained by the area contraction. At the entrance of the combustion chamber $(x=$ $0.50 \mathrm{~m}$ ) there is an area expansion, but no obvious discontinuity can be observed here. This can be explained by the fact that the velocity decrease due to the area expansion is compensated by the increase due to the change in speed of sound. The location of the unsteady heat release source is indicated by the vertical gray lines. The mode shapes of the operating point at $M a=0.034$ and $\Phi=0.78$ are similar and are not provided here.

\section{Unsteady heat release}

As described in section 2.2.5, the next step when solving for the system stability consists of solving the temporal ordinary differential equation, which includes all damping mechanisms as well as the unsteady heat release of the flame in the source terms. Hield et al. determined their Flame Describing Function by combining their Fourier-transformed acoustic velocity measurements with their Fourier-transformed 

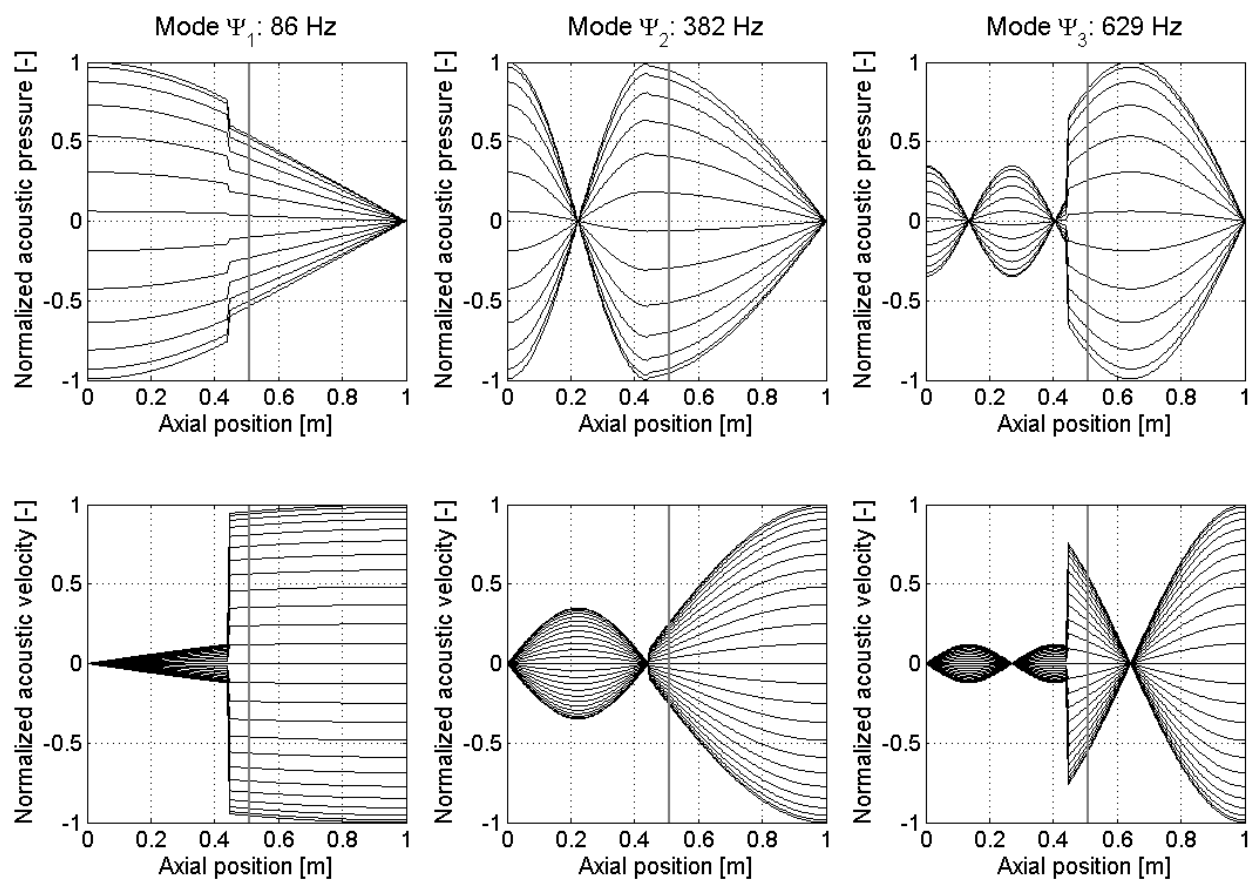

Figure 2.3: Calculated normalized pressure and velocity mode shapes for the bluff body burner (Operating point $M a=0.04, \Phi=1$ ). Vertical gray lines (at $x=0.50 \mathrm{~m}$ ) indicate location of unsteady heat release.

$C H^{*}$ measurements. They determined the FDF in such a way that it fitted their data both as function of frequency and as function of the root mean square velocity amplitude. Hield et al.'s curve fit is:

$$
\mathcal{F}\left(\omega,\left|u^{\prime}\right|\right)=\underbrace{\left[1.6 e^{-0.2\left|u^{\prime}\right|}+0.225\right]}_{\text {Dependency on rms-velocity }} \underbrace{\frac{\omega_{c o}^{2}}{\omega_{c o}^{2}+2 i \zeta \omega_{c o} \omega-\omega^{2}}}_{\text {Low pass filter }} \underbrace{e^{-i \omega \tau}}_{\text {Time lag }}
$$

The first term inside the brackets provides the nonlinearity to the model and represents the dependency of the Flame Describing Function on the acoustic velocity amplitude. The second part of the model represents the frequency dependency of the FDF. It was noticed in experiments that the response of the flame decreased with increasing frequency. Hield et al. [41] fitted this behavior with a low pass filter, with cut-off frequency $\omega_{c o}=350 \mathrm{~Hz}$ and damping coefficient $\zeta=0.5$. The Flame Describing Function according to equation 2.19 is graphically presented in figure 2.4, as a function of frequency and for different rms-velocity amplitudes. It can clearly be seen that the FDF obtains smaller values for increasing rms-velocity amplitudes, which means that the flame saturates. It can also be seen in figure 2.4 that the FDF decreases for frequencies larger than the cut-off frequency. 

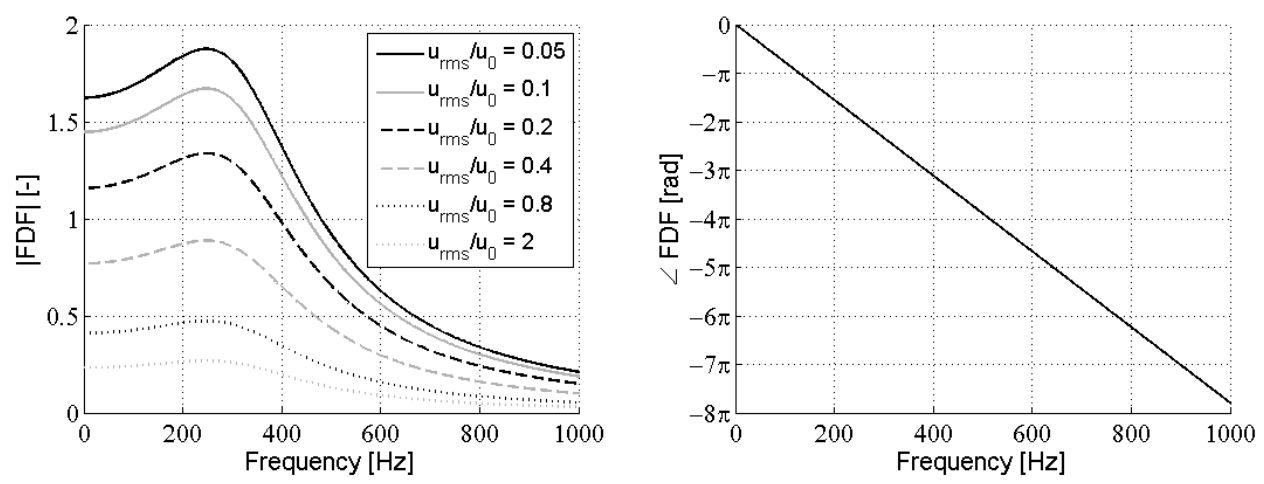

Figure 2.4: The Flame Describing Function according to the flame model of Hield et al. [41]. The left plot shows the gain for different velocity amplitudes, the right plot shows the phase for $\tau=3.9 \mathrm{~ms}$. Note that the phase is independent of velocity amplitude.

\begin{tabular}{lcccc}
\hline Operating point & Time lag & $\angle \mathcal{F}\left(\mathbf{f}_{\mathbf{1}}\right)$ & $\angle \mathcal{F}\left(\mathbf{f}_{\mathbf{2}}\right)$ & $\angle \mathcal{F}\left(\mathbf{f}_{\mathbf{3}}\right)$ \\
\hline$M a=0.040, \Phi=1$ & $3.9 \mathrm{~ms}$ & $-0.68 \pi$ & $-3.10 \pi$ & $-4.74 \pi$ \\
\hline$M a=0.034, \Phi=0.78$ & $5.9 \mathrm{~ms}$ & $-1.01 \pi$ & $-4.47 \pi$ & $-7.15 \pi$ \\
\hline
\end{tabular}

Table 2.2: Time lags and phase for the bluff body stabilized burner.

Finally the last term $e^{-i \omega \tau}$ in equation 2.19 represents the time delay between velocity and heat release fluctuations. The time lag is a very important term, since it determines whether the flame damps or excites acoustic waves. The time lags which have been used for the different cases presented here were obtained from measured data and are given in table 2.2. The phase difference between velocity and heat release fluctuations for each acoustic mode $(\angle \mathcal{F}=-2 \pi f \tau)$ has been calculated as well. The phase can be interpreted as a dimensionless time lag.

According to Rayleigh's criterion, see section 1.2, instabilities can be expected when the phase difference between pressure fluctuations and heat release fluctuations lies between $\left[-\pi / 2<\angle p^{\prime} q^{\prime}<\pi / 2\right]$. For heat release models expressed in $Q^{\prime} \propto u^{\prime}$ form however, the phase difference between pressure and velocity fluctuations has to be added to this range in order to determine the stable and unstable regimes. This phase difference $\angle p^{\prime} u^{\prime}$ is geometry dependent, it depends on the boundary conditions and the axial position of the heat release source. Later in this chapter a sensitivity study on the phase of the FDF will be performed and the stable and unstable ranges will be determined.

\section{Boundary conditions}

As previously mentioned, the main damping mechanisms are the boundary conditions. Hield et al. assumed that the open outlet boundary condition is fully reflective $(R=-1)$ and does not provide any damping. On the other hand, the inlet boundary is choked and some acoustic energy can leave the domain. According to 
Dowling [24], the reflection coefficient for a choked inlet is:

$$
R=\frac{1-M a}{1+M a}
$$

The reflection coefficients based on these equations can be found in table 2.3.

\begin{tabular}{lcc}
\hline Operating point & $\mathbf{R}_{\text {inlet }}$ & $\mathbf{R}_{\text {outlet }}$ \\
\hline$M a=0.040, \Phi=1$ & 0.92 & -1 \\
$M a=0.034, \Phi=0.78$ & 0.93 & -1 \\
\hline
\end{tabular}

Table 2.3: Boundary conditions for bluff body burner operating points.

\section{Results for the operating point $M a=0.040, \Phi=1$}

To illustrate the physics described previously using figure 1.4 of Chapter 1, first a simulation with a fully reflective boundary condition at the inlet $(R=1)$ instead of the choked boundary condition has been run. The results are shown in figure 2.5(a). It can clearly be seen that the growth rate of the pressure amplitude shows nonlinear behavior, but a limit cycle is not obtained. This is because the gain of the FDF given by equation 2.19 does not become zero for large values of the root mean square acoustic pressure. On the other hand, if only a choked inlet boundary condition is considered but no heat release, the acoustics damp out (see figure 2.5(b)). However, the combination of both the nonlinear heat release model and the choked inlet boundary condition does lead to a limit cycle, this is shown in figure 2.5(c).

\begin{tabular}{lccc}
\hline & Frequency [Hz] & Pressure [-] & Velocity [-] \\
\hline Simulation & 395 & $p^{\prime} / p_{0}=0.28$ & $u^{\prime} / u_{0}=4.4$ \\
Measurement & 364 & $p^{\prime} / p_{0}=0.25$ & $u^{\prime} / u_{0}=4.0$ \\
\hline
\end{tabular}

Table 2.4: Comparison simulation and measurement for the second mode of the bluff body burner $(M a=0.04, \Phi=1)$.

Hield et al. [41] measured a limit cycle for the second eigenmode of the combustor. The modeled limit cycle, plotted in figure 2.5(c), has a normalized pressure amplitude of $p^{\prime} / p_{0}=0.28$, which is very close to the measured value of $p^{\prime} / p_{0}=0.25$. A zoom in of the pressure trace is presented in figure 2.5(e). The non dimensional limit cycle velocity amplitude, shown in figure $2.5(\mathrm{~d})$, is about 4.4 , for which a value of $u^{\prime} / u_{0}=4.0$ was measured. This means that significant flow reversal occurs, making this the most likely cause for the saturation of the flame.

The damping and growth mechanisms changed the frequency of the second mode $\Psi_{2}$ from 382 to $395 \mathrm{~Hz}$. The measured frequency of the limit cycle was $f=364 \mathrm{~Hz}$, so the Generalized Instability Model over predicts this by $31 \mathrm{~Hz}(8 \%)$. A reason for this overprediction could be the fact that the combustor (domain III of the GIM model) has been assumed to have the adiabatic flame temperature. As discussed 


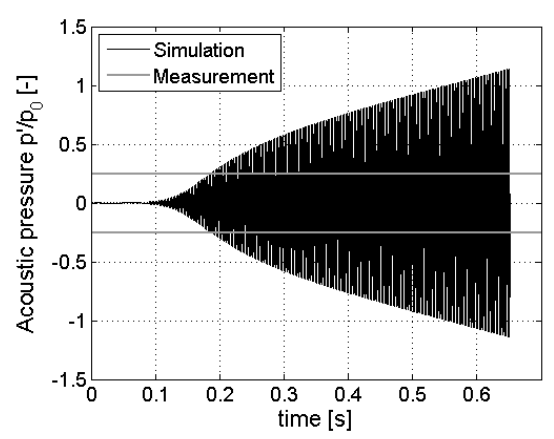

(a) Flame Describing Function \& fully reflective boundary conditions $(R=1)$ : No limit cycle can be obtained.

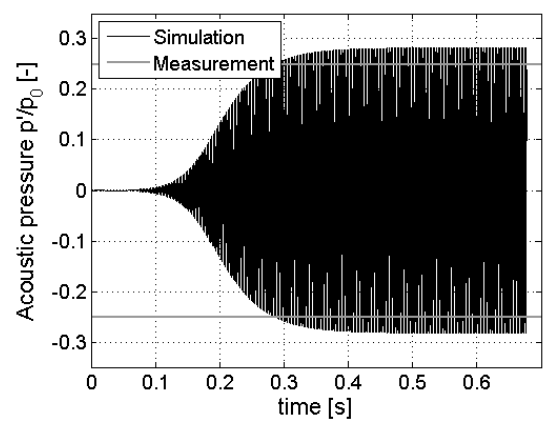

(c) Flame Describing Function \& choked nozzle inlet boundary condition: Limit cycle pressure amplitude.

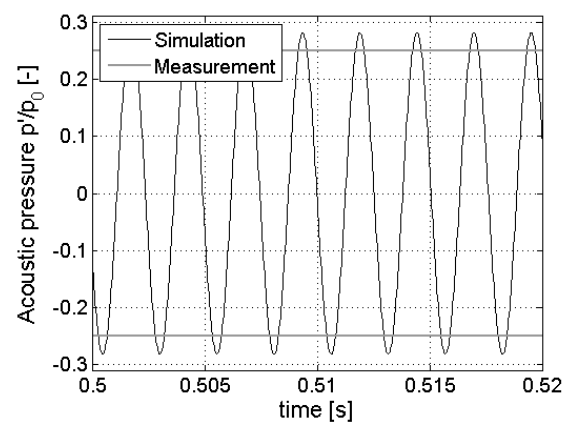

(e) Zoom in on figure 2.5(c): Limit cycle pressure amplitude.

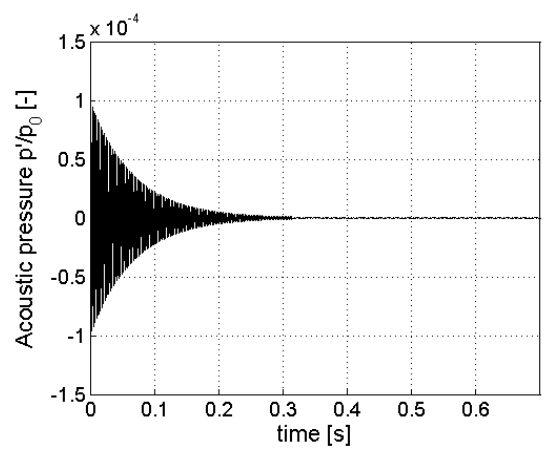

(b) No heat release \& choked nozzle inlet boundary condition (Table 2.3): The acoustic oscillations damp out.

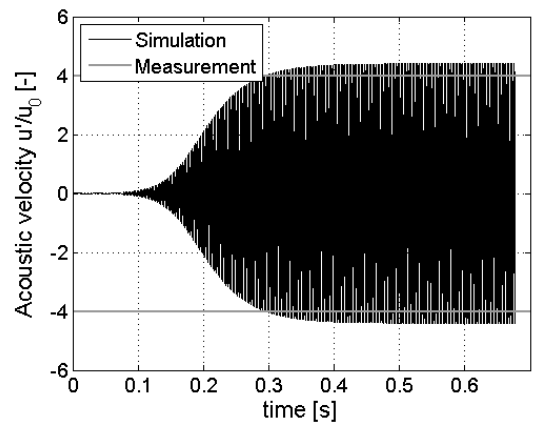

(d) Flame Describing Function \& choked nozzle inlet boundary condition: Limit cycle velocity amplitude.

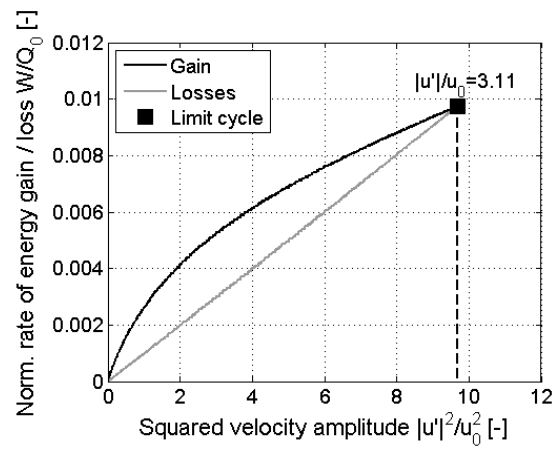

(f) Balance between energy gain and losses.

Figure 2.5: Simulated results for the second mode of the bluff body burner $(M a=0.04$, $\Phi=1$ ). Note: different scales on the y-axis have been used for the various plots. 
in the introduction, it is assumed by the Galerkin approach that the damping and growth mechanisms perturb the modes, but do not change the mode shapes themselves. This may be a second reason. Nonetheless, the agreement between the GIM simulation and the measured data is considered to be reasonably good.

Simulations for the modes $\Psi_{1}(f=86 \mathrm{~Hz})$ and $\Psi_{3}(f=629 \mathrm{~Hz})$ have been run as well. These modes were not observed in measurements, meaning they are stable modes. For the mode $\Psi_{1}$, GIM predicts a growth rate of $\Gamma=-122 \mathrm{rad} / \mathrm{s}$ and for the mode $\Psi_{3}$, GIM predicts $\Gamma=-28 \mathrm{rad} / \mathrm{s}$. A negative growth rate means that these modes damp out, which agrees with findings from the measurements.

Using the results from the simulations, an energy balance diagram similar to figure 1.4 of Chapter 1 can be created for the unstable mode $\Psi_{2}$. First the acoustic energy is introduced (see Poinsot [75]):

$$
e^{\prime}=\frac{1}{2} \rho_{0}\left(\mathbf{u}^{\prime} \cdot \mathbf{u}^{\prime}\right)+\frac{1}{2} \frac{p^{\prime 2}}{\rho_{0} c^{2}}
$$

Differentiating equation 2.21 with respect to time, substituting the linearized Euler equations (equation 2.4 and 2.5) for $\partial p^{\prime} / \partial t$ and $\partial u^{\prime} / \partial t$ and neglecting mean flow contributions leads to a balance equation for the acoustic energy:

$$
\frac{\partial e^{\prime}}{\partial t}+\nabla \cdot\left(p^{\prime} \mathbf{u}^{\prime}\right)=\frac{\gamma-1}{\gamma p_{0}} p^{\prime} q^{\prime}
$$

More detailed discussions on the energy contained in fluctuating properties of turbulent reacting flows can be found in Nicoud et al. [69] or George et al. [33]. Integrating equation 2.22 over the volume finally leads to:

$$
\frac{\partial E^{\prime}}{\partial t}=\int_{V} \frac{\partial e^{\prime}}{\partial t} d V=W_{\text {gain }}-W_{\text {loss }}
$$

with

$$
\begin{aligned}
W_{\text {gain }} & =\int_{V} \frac{\gamma-1}{\gamma p_{0}} p^{\prime} q^{\prime} d V=\frac{\gamma-1}{\gamma p_{0}} p^{\prime}\left(x_{f}\right) Q^{\prime} \\
W_{\text {loss }} & =\int_{V} \nabla \cdot\left(p^{\prime} \mathbf{u}^{\prime}\right) d V=\int_{A} p^{\prime} \mathbf{u}^{\prime} \cdot \mathbf{n} d A
\end{aligned}
$$

Here $W_{\text {gain }}$ is the rate of acoustic energy produced by the combustion process and $W_{\text {loss }}$ the acoustic energy losses. In equation 2.24, $p^{\prime}\left(x_{f}\right)$ stands for the pressure at the location of the flame. Furthermore in equation 2.25 the divergence theorem has been used, from which it can be concluded that the losses need to be integrated over the area $A$ of the walls of the geometry. It should further be noted that $W_{\text {gain }}$ and $W_{\text {loss }}$ correspond to the time derivatives of the the modified Rayleigh criterion, introduced in Chapter 1 and given by equation 1.2.

In figure 2.5(f) the energy gain and losses calculated by GIM are shown as a function of the squared velocity amplitude. Both $W_{\text {gain }}$ and $W_{\text {loss }}$ have been normalized by the mean heat release $Q_{0}$. The energy balance of figure 2.5(f) looks similar to the sketch in figure 1.4, although it does not really contain a linear regime. The reason 
for this is that the particular Flame Describing Function that has been used (given by equation 2.19) is already nonlinearly dependent on $\left|u^{\prime}\right|$ for very small velocity amplitudes. Also it can be seen that the plot does not contain data for $\left|u^{\prime}\right|>\left|u^{\prime}\right|$ Limit cycle (which would only be possible if the initial amplitude were larger than the limit cycle amplitude). It can be seen that for the limit cycle $W_{\text {gain }} / Q_{0} \approx 0.01$, meaning that about $1 \%$ of the total heat released by the flame is converted into acoustic energy.

\section{Results for the operating point $M a=0.034, \Phi=0.78$}

The main difference between this operating point and the operating point with $\Phi=1$ is the larger time lag between velocity fluctuations at the bluff body (see table 2.2) and heat release fluctuations. Also the total heat release $Q_{0}$ is smaller, due to the lower equivalence ratio and mass flow. The temperature of the combustion chamber was slightly lower as well (see table 2.1). The growth rates that have been calculated with GIM for this operating condition are tabulated in table 2.5:

\begin{tabular}{lll}
\hline Mode & Phase & Growth rate \\
\hline$\Psi_{1}=86 \mathrm{~Hz}$ & $\angle \mathcal{F}=-1.01 \pi$ & $\Gamma=-14 \mathrm{rad} / \mathrm{s}$ \\
$\Psi_{2}=379 \mathrm{~Hz}$ & $\angle \mathcal{F}=-4.47 \pi$ & $\Gamma=-99 \mathrm{rad} / \mathrm{s}$ \\
$\Psi_{3}=606 \mathrm{~Hz}$ & $\angle \mathcal{F}=-7.15 \pi$ & $\Gamma=+4 \mathrm{rad} / \mathrm{s}$ \\
\hline
\end{tabular}

Table 2.5: Results for the second operating point of the bluff body burner $(M a=0.034$, $\Phi=0.78)$.

From table 2.5 it can be concluded that the first and the second mode are stable. The third mode was found to be neutrally stable (the growth rate is positive but very small), indicating that the energy losses through the boundaries and the energy gain by the flame approximately compensate each other.

No thermo-acoustic instabilities were found in the measurements for this operating point. For neutrally stable modes, it is difficult to interpret the simulated results because sensitivity to small errors in time lag, boundary conditions, etc., may change a small positive growth rate to a small negative one. Also damping mechanisms like turbulent dissipation (which is not modeled in GIM) may become important for small growth rates. This suggests that a sensitivity analysis on these parameters is important, as will be shown in the next paragraph.

\section{Sensitivity analysis}

It is now interesting to investigate the sensitivity of the boundary conditions and the Flame Describing Function on the predicted limit cycle pressure amplitude. First the influence of the FDF on the system stability has been determined. In order to test this, the phase of the FDF has been varied for both operating conditions. The gain of the FDF has not been varied in this study and corresponds to the gain of the second eigenmode of the combustor (approximately $380 \mathrm{~Hz}$ ).

In figure 2.6 the predicted limit cycle pressure amplitude as a function of the phase / time lag is shown. The baseline results according to the measured time lags 
for both operating conditions are indicated as well. It can be seen that the unstable range is approximately given by $[-4 \pi<\angle \mathcal{F}<-3 \pi]$, whereas the ranges $[-3 \pi<$ $\angle \mathcal{F}<-2 \pi]$ and $[-5 \pi<\angle \mathcal{F}<-4 \pi]$ are stable ranges.

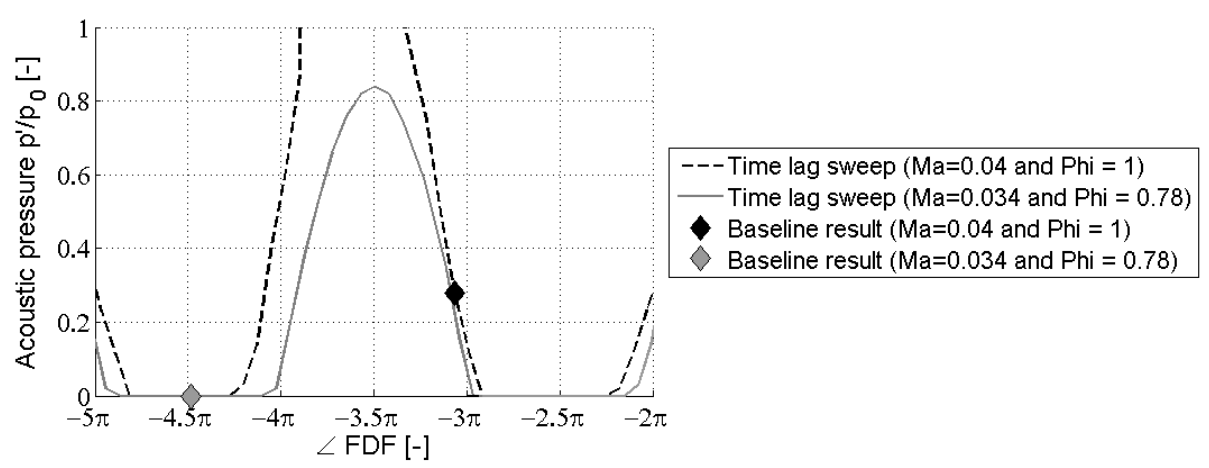

Figure 2.6: Influence of the phase between velocity and heat release fluctuations $(\angle \mathcal{F}=$ $-2 \pi f \tau$ ) on the limit cycle pressure amplitude. The time lag $\tau$ has been varied here, for the second eigenmode (approximately $380 \mathrm{~Hz}$ ) of both operating points. The markers show the baseline results according to the measured time lags (see previous paragraphs).

For the stable operating condition ( $M a=0.034$ and $\Phi=0.78)$, the exact value of the phase is not very important, because the operating condition is stable for the complete range $[-5 \pi<\angle \mathcal{F}<-4 \pi]$. For unstable conditions however, the predicted limit cycle pressure amplitude is very sensitive on the phase, as can be seen from the steep slope of the acoustic pressure between $-4 \pi$ and $-3 \pi$. For the operating point $M a=0.04$ and $\Phi=1$ there even is a range (approximately $[-3.8 \pi<\angle \mathcal{F}<-3.3 \pi]$ ) in which the pressure amplitude grows out of bounds and no limit cycle is found. For these cases, when plotted in an energy balance diagram (e.g. figure 2.5(f)), the slope of the gain curve in the energy balance would exceed the slope of the damping curve even for large $\left|u^{\prime}\right|$, which can be explained by the fact that Hield's Flame Describing Function (see equation 2.19) converges to a constant value for large values of $\left|u^{\prime}\right|$.

Next, the influence of the losses has been determined. The baseline results have been obtained using the reflection coefficients at the inlet and the outlet according to table 2.3. In figure 2.7, a sensitivity analysis on both the inlet and outlet reflection coefficient is presented. It can be seen that the reflection coefficient also has a large influence on the results. Especially when the reflection coefficient of the inlet is increased (less losses through the boundaries), the limit cycle pressure amplitude rapidly increases. For decreasing reflection coefficient (more losses through the boundaries), a slower reduction of the pressure amplitude with reflection coefficient can be observed.

\subsubsection{Additional validation cases}

In Krediet et al. [56], validation data for additional operating conditions of Hield's bluff body stabilized burner can be found. Also data for a swirl stabilized flame is provided in this paper. These cases show similar results as the results provided 


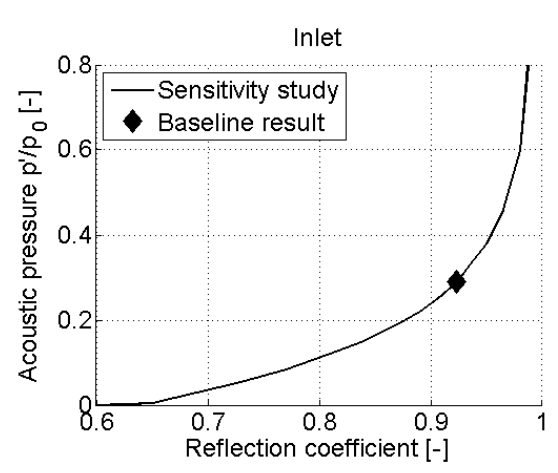

(a) Influence of the inlet reflection coefficient ( $R=1$ represent a fully reflecting fixed velocity boundary condition, without losses). The outlet reflection coefficient was kept constant in this analysis $\left(R_{\text {outlet }}=-1\right)$.

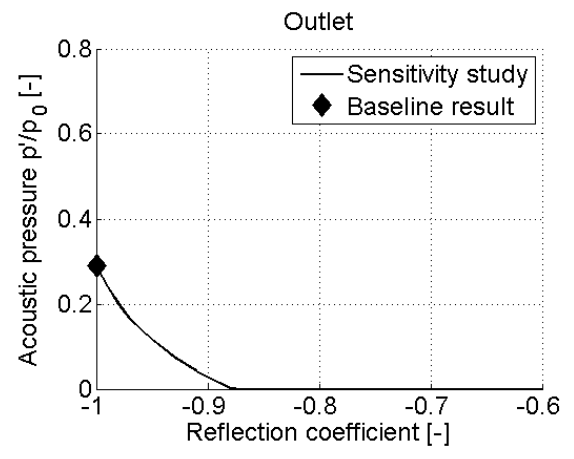

(b) Influence of the outlet reflection coefficient ( $R=-1$ represent a fully reflecting fixed pressure boundary condition, without losses). The inlet reflection coefficient was kept constant in this analysis $\left(R_{\text {inlet }}=0.92\right)$.

Figure 2.7: Influence of the boundary condition reflection coefficient on the limit cycle pressure amplitude for the operating point with $M a=0.04$ and $\Phi=1$. The markers show the baseline results according to Dowling's analytical expression for a choked inlet (equation 2.20).

in this chapter and the same conclusions can be drawn from them. Therefore these results are not shown in this dissertation. In Chapter 4 and 5 however, additional results obtained with the nonlinear stability tool will be shown. These results will be obtained using Flame Describing Functions obtained via CFD simulations.

\subsection{Conclusions}

In this chapter, a nonlinear thermo-acoustic system model for the prediction of limit cycles has been proposed and validated with measurement data from a bluff body stabilized burner. For all operating points which have been modeled, the linear stability was successfully predicted. For the unstable points, the limit cycle pressure amplitudes were successfully predicted as well.

An important conclusion from this validation work is that the accuracy in the prediction of the limit cycle pressure amplitude is sensitive to both the Flame Describing Function (FDF) and the acoustic boundary conditions. A parametric analysis showed that changes of the time lag of less than $10 \%$ of the period can double the limit cycle amplitude. In addition, the reflection coefficient has a major impact on the predicted limit cycle amplitude as well. This shows that that both the FDF and acoustic energy losses should be accurately predicted in order to assess a certain configuration. It can also be concluded that the results from a nonlinear limit cycle calculation are only relevant when this sensitivity is taken into account, since changes may be large for small changes in time lag and or boundary conditions.

The validation results provided in this chapter have been obtained using measured Flame Describing Functions. In the next chapters, the FDF will be obtained 
from Computational Fluid Dynamics simulations. The next step is then to validate the prediction of limit cycle pressure amplitudes using the FDF from simulations. This will be done in Chapter 4 and 5 .

It would be possible to answer Research question 1 (see Chapter 1, section 1.3) using the results obtained in this chapter. However, an answer to this research question will first be provided in the Conclusions and Recommendations chapter, Chapter 6, so that the additional results obtained in 4 and 5 can also be taken into account. 


\section{3}

Transient CFD simulation of the reacting flow

\subsection{Introduction}

In this work, the Flame Describing Function (FDF), discussed in the previous chapters, will be obtained from Computational Fluid Dynamics (CFD) simulations. In order to obtain the FDF, a steady simulation is excited at its boundaries to identify its dynamic response. The procedure consists of the following steps $[51,76,77]$ :

1. Obtain a stable and converged reacting flow solution, without excitation of the boundaries. The initial phenomena should have decayed.

2. Perform a transient CFD simulation, in which the boundary conditions are excited. The acoustic quantities of interest (pressure, velocity, mixture fraction and heat release) are being sampled on specific sampling planes.

3. Reconstruct the Flame Describing Function from the time series of the sampled quantities.

The procedure to identify Flame Transfer Functions from CFD has already been validated by several other researchers, e.g. Armitage et al. [4], van Kampen [47] and Polifke et al. [12,76]. In this work, the procedure will be extended to Flame Describing Functions.

The work done within this work is performed using a SIEMENS in-house developed CFD code for turbulent reacting flows, which is an extension of the Open-source CFD code OpenFoam [1]. This chapter is structured as follows: first the governing 
equations solved by the CFD code are introduced and motivated (Section 3.2). In the next section (Section 3.3), the specific issues for the transient CFD simulation with the excitation of the boundary conditions are discussed. These are for instance the setup of the boundary conditions and the sampling of acoustic quantities. Also the reconstruction procedure of acoustic transfer functions such as the Flame Describing Function will be described.

The application of the SIEMENS CFD code to reacting flows is not new (see for instance di Domenico et al. [23]). However, the application of the code to thermoacoustic problems, in which the boundary conditions are perturbed, has been developed within this work. For this reason, before applying the CFD code to complicated geometries and including combustion, the functionality of the code for thermoacoustic applications has first been verified using simple, non-reacting test cases. This verification is presented in section 3.4. The application of the code to real gas turbine combustors will be done in Chapter 4 and Chapter 5 .

\subsection{Equations governing turbulent reacting flow}

The flow field through a typical gas turbine burner is highly turbulent. Turbulence is described by the Navier-Stokes equations, so a turbulent flow could be calculated by solving the Navier Stokes equations in a direct way. This is called Direct Numerical Simulation (DNS). However, for engineering computations, this method requires far too much computational effort to be solved on any foreseeable computer. The ratio between the smallest turbulent structures (eddies) present in the flow (the Kolmogorov scale $\eta_{K o l}$ ) and the length scale of the largest turbulent eddies $L$ is related to the Reynolds number $R e$ according to (see e.g. [80]):

$$
\eta_{K o l} / L=\mathcal{O}\left(R e^{-3 / 4}\right)
$$

In order to resolve the Kolmogorov scale, the mesh size $\Delta x$ of the computational grid must satisfy $\Delta x<\eta_{K o l}$. This implies that the number of grid cells $N$ is given by:

$$
N>L / \Delta x=\mathcal{O}\left(R e^{3 / 4}\right)
$$

In a three-dimensional simulation the number of cells is $\mathcal{O}\left(R e^{9 / 4}\right)$. With typical Reynolds numbers for gas turbine applications in the order of $R e=\mathcal{O}\left(10^{5}\right)$ or higher, DNS is inappropriate for engineering applications. Therefore, DNS is mainly used for studying the fundamentals of turbulence in simple geometries.

In general there are two other methods which try to decrease the computational effort by incorporating some additional turbulence model: Large Eddy Simulation (LES) and a solution based on solving the Reynolds averaged Navier-Stokes (RANS) equations. In LES, the larger turbulent eddies are resolved numerically and only the smaller eddies are modeled. Here it is assumed that the structure of the larger eddies is dependent on the particular geometry, but the structure of the small eddies is to a large extent independent of it and universal.

In RANS on the other hand, all turbulent structures are modeled. This is done by averaging the Navier Stokes equations. Models are provided to account for the 
influence of the averaged turbulent fluctuations on the mean flow field. As a result, solving the RANS equations requires much less computational effort than the LES approach, but the price that has to be paid for this speed up is that the mean flow field solutions are generally not as accurate as the solutions obtained via LES.

The difference between DNS, RANS and LES can clearly be seen in figure 3.1 and 3.2. Figure 3.1 schematically shows the time evolution of the local temperature in a turbulent flame, where as in figure 3.2 the turbulent energy spectrum is shown. In figure $3.2, k_{c}$ is the cut-off wave number. It is a measure for the part of the turbulent eddies that are computed and the part that is modeled and is dependent on the grid size.

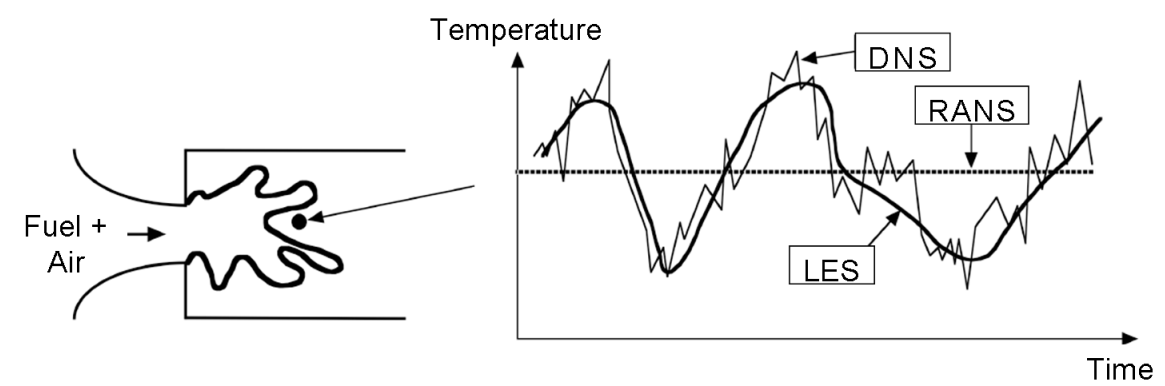

Figure 3.1: Time evolution of local temperature computed with DNS, RANS or LES in a turbulent flame. Sketch taken from Poinsot et al. [75]

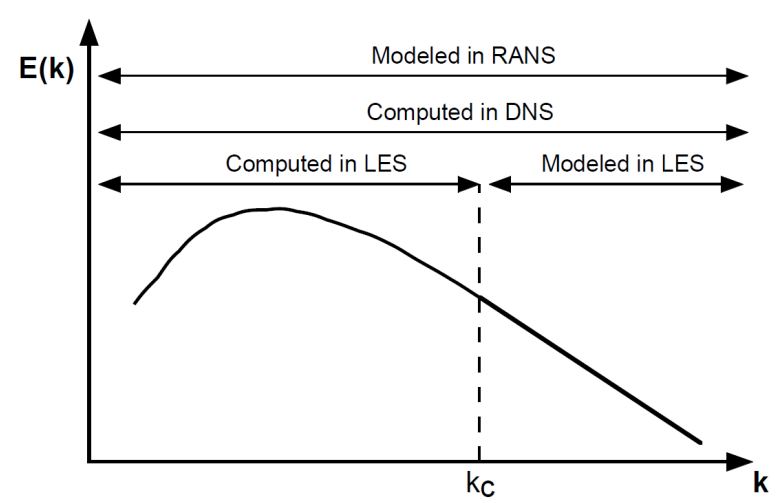

Figure 3.2: Turbulence energy spectrum as a function of the wave number. RANS, LES and DNS are summarized in terms of spatial frequency range. $k_{c}$ is the cut-off wave number used in LES and is dependent on the grid size. Sketch taken from Poinsot et al. [75]

Besides RANS and LES, some other approaches include URANS, which is able to solve unsteady phenomena with very large time scales (such as acoustic fluctuations). Another approach is Scale Adaptive Simulation (SAS), which is a hybrid approach. Based on the flow regime, the approach switches between RANS and LES modeling.

The simulations in this work are performed using Large Eddy Simulation. Al- 
though this approach is more expensive than RANS, it gives the highest confidence that all relevant physics that may have an impact on the Flame Describing Function are actually being resolved. Thermo-acoustic oscillations are unsteady phenomena, triggered by unsteadiness in the flow, and in LES all unsteady flow structures are directly solved.

\subsubsection{Introduction to LES}

In Large Eddy Simulations, the mean value of a quantity $f$ over a given cell is solved. This means that all variables are filtered in physical space. The filtered quantity $\bar{f}$ is defined as:

$$
\bar{f}(\mathbf{x}, t)=\int f\left(\mathbf{x}^{\prime}, t\right) F\left(\mathbf{x}-\mathbf{x}^{\prime}\right) \mathbf{d} \mathbf{x}^{\prime}
$$

where $F$ is the LES filter. An often used approach is averaging the variables over a cubic box of size $\Delta$, where

$$
F(\mathbf{x})= \begin{cases}1 / \Delta^{3} & \text { if }\left|\mathbf{x}-\mathbf{x}^{\prime}\right|<\Delta / 2, \\ 0 & \text { otherwise }\end{cases}
$$

Here, $\Delta$ is defined as the cubic root of the local cell volume. For variable density flow, a density-weighted Favre filtering is used as well:

$$
\bar{\rho} \tilde{f}(\mathbf{x}, t)=\int \rho f\left(\mathbf{x}^{\prime}, t\right) F\left(\mathbf{x}-\mathbf{x}^{\prime}\right) \mathbf{d} \mathbf{x}^{\prime}
$$

So $\tilde{f}(\mathbf{x}, t)$ is the density-weighted mean value of $f$ over a given cell. The quantity $f^{\prime \prime}$ on the other hand corresponds to the unresolved part within a given cell and needs to be determined using a sub grid turbulence model.* It should be noted that in LES calculations the smaller the cell size $\Delta$, the larger is the part of the turbulent spectrum that is resolved and the smaller the part that needs to be modeled. In the limit of $\Delta x<\eta_{K o l}$, Large Eddy Simulation converges to DNS.

\subsubsection{LES Filtered Navier Stokes equations for turbulent reacting flow}

The generalized version of the LES Filtered Navier Stokes equations for reacting flow is given below. Details of the derivation can be found in Poinsot et al. [75].

*For turbulent sub grid fluctuations a notation using double apostrophes is used (e.g. $\left.u^{\prime \prime}\right)$. These sub grid fluctuations should not be confused with acoustic fluctuations (e.g. $\left.u^{\prime}\right)$, which are represented by a single apostrophe. In LES, acoustic fluctuations are fully resolved. 
Mass: *

$$
\frac{\partial \bar{\rho}}{\partial t}+\nabla \cdot(\bar{\rho} \widetilde{\mathbf{u}})=0
$$

Momentum:

$$
\frac{\partial(\bar{\rho} \widetilde{\mathbf{u}})}{\partial t}+\nabla \cdot(\bar{\rho} \widetilde{\mathbf{u}} \otimes \widetilde{\mathbf{u}})=-\nabla \bar{p}+\nabla \cdot \overline{\bar{\tau}}-\nabla \cdot\left(\bar{\rho} \overline{\mathbf{u}^{\prime \prime} \mathbf{u}^{\prime \prime}}\right)
$$

Species mass fraction:

$$
\frac{\partial\left(\bar{\rho} \widetilde{Y_{k}}\right)}{\partial t}+\nabla \cdot\left(\bar{\rho} \widetilde{\mathbf{u}} \widetilde{Y_{k}}\right)=\nabla \cdot\left(\bar{\rho} \overline{D_{k}} \nabla \widetilde{Y_{k}}\right)-\nabla \cdot\left(\bar{\rho} \overline{\mathbf{u}^{\prime \prime} Y_{k}^{\prime \prime}}\right)+\widetilde{w_{k}}
$$

Total enthalpy:

$$
\frac{\partial(\bar{\rho} \widetilde{h})}{\partial t}+\nabla \cdot(\bar{\rho} \widetilde{\mathbf{u}} \tilde{h})=\frac{D \bar{p}}{D t}+\overline{\tau: \nabla \mathbf{u}}+\nabla \cdot(\overline{\lambda \nabla T})-\nabla \cdot\left(\overline{\rho \sum_{k=1}^{N} h_{k} \nabla Y_{k}}+\bar{\rho} \overline{\mathbf{u}^{\prime \prime} h^{\prime \prime}}\right)
$$

where

$$
\begin{aligned}
h & =\sum_{k=1}^{N} h_{k} Y_{k} \\
h_{k} & =h_{k}^{0}+\int_{T_{0}}^{T_{1}} C_{P, k} d T
\end{aligned}
$$

In equations 3.6 to $3.8, \bar{\rho}$ is the density, $\tilde{\mathbf{u}}$ the velocity, $\bar{p}$ the pressure, $\widetilde{Y_{k}}$ the species mass fraction, $D_{k}$ the diffusivity of species $k$ and $\overline{\bar{\tau}}$ the viscous stress tensor. The viscous stress tensor $\overline{\bar{\tau}}$ is given by equation 3.12. The source term $\widetilde{\dot{w}_{k}}$ in the species mass fraction equation represents the mass conversion rate of species $k$.

$$
\overline{\bar{\tau}}=\left[\mu\left(\nabla \tilde{\mathbf{u}}+\nabla \tilde{\mathbf{u}}^{T}\right)-\frac{2}{3} \mu(\nabla \cdot \tilde{\mathbf{u}}) \mathbf{I}\right]
$$

with $\mu$ the laminar viscosity.

In equations 3.9 to $3.11, \widetilde{h}$ is the total enthalpy, $T$ the temperature, $\lambda$ the thermal conductivity and $C_{P}$ the specific heat capacity. It should be noted that equation 3.9 does not have a source term. The enthalpy change due to chemical reactions is taken into account by equations 3.10 and 3.11 , where $h_{k}^{0}$ is the enthalpy of formation.

The enthalpy equation can be greatly simplified by assuming [22]: scalar:

*In this dissertation, a bold printed quantity (e.g. $\widetilde{\mathbf{u}})$ represents a vector, where as $\widetilde{u_{x}}$ represents a

$$
\nabla \cdot(\bar{\rho} \widetilde{\mathbf{u}})=\frac{\partial\left(\bar{\rho} \widetilde{u_{x}}\right)}{\partial x}+\frac{\partial\left(\bar{\rho} \widetilde{u_{y}}\right)}{\partial y}+\frac{\partial\left(\bar{\rho} \widetilde{u_{z}}\right)}{\partial z}
$$


- Energy of the viscous stresses can be neglected compared to the heat release by combustion. This assumption causes the second term on the right hand side of equation 3.9 to vanish.

- Thermal diffusion can be ignored, the mean molar mass is assumed to be constant and the Lewis number $L e=\lambda /\left(\rho C_{P} D\right)$ is unity for all species. This causes the third and fourth term on the r.h.s. of equation 3.9 to cancel each other.

This leads to a simpler form of the enthalpy equation:

$$
\frac{\partial(\bar{\rho} \widetilde{h})}{\partial t}+\nabla \cdot(\bar{\rho} \widetilde{\mathbf{u}} \widetilde{h})=\frac{D \bar{p}}{D t}-\nabla \cdot\left(\bar{\rho} \overline{\mathbf{u}^{\prime \prime} h^{\prime \prime}}\right)
$$

In many gases $L e$ is very close to unity, it is often slightly less than unity in combustible gas mixtures [22].

In order to solve the set of equations above, the followings terms have first to be closed:

- The unresolved terms $\bar{\rho} \overline{\mathbf{u}^{\prime \prime} \mathbf{u}^{\prime \prime}}, \bar{\rho} \overline{\mathbf{u}^{\prime \prime} Y_{k}^{\prime \prime}}$ and $\bar{\rho} \overline{\mathbf{u}^{\prime \prime} h^{\prime \prime}}$. These terms represent the sub grid fluctuations of momentum, species mass fraction and enthalpy and have to be modeled using a turbulence model. The turbulence model will be discussed in section 3.2.3.

- The chemical source term $\widetilde{\dot{w}_{k}}$ of the species mass fraction transport equations. For methane-air combustion, the GRI 3.0 mechanism consists of 53 species $Y_{k}$ and 325 reactions. Solving this mechanism using LES would be very expensive. Therefore the species mass fraction transport equations and its source term have to be simplified using a combustion model. The combustion model is discussed in section 3.2.4.

\subsubsection{Turbulence model}

In the set of equations derived above (equations 3.6 to 3.13), the Favre averaging produces products between fluctuating quantities. These terms have to be resolved using sub grid models.

The first unresolved term is the term $\mathbf{T}=\bar{\rho} \overline{\mathbf{u}^{\prime \prime} \mathbf{u}^{\prime \prime}}$ in the momentum equation (also called the Reynolds stress). The term causes additional momentum transfer due to sub grid turbulent fluctuations. At high Reynolds numbers and remote from walls, this term is often much larger than the viscous stresses [75]. Different LES turbulence models have been proposed for the modeling of this term, usually these models are based on fitting data obtained from DNS or measurements. One of the most applied turbulence models for LES is the Smagorinsky model $[75,93]$. This model will be used in this work. In this Smagorinsky model, the Reynolds stress is modeled as:

$$
T_{i j}-\frac{\delta_{i j}}{3} T_{k k}=-\mu_{T}\left(\frac{\partial \widetilde{u_{i}}}{\partial x_{j}}+\frac{\partial \widetilde{u_{j}}}{\partial x_{i}}\right)=-2 \mu_{T} \widetilde{S_{i j}}
$$


with $S_{i j}=\frac{1}{2}\left(\frac{\partial \widetilde{u_{i}}}{\partial x_{j}}+\frac{\partial \widetilde{u_{j}}}{\partial x_{i}}\right)$. The turbulent viscosity $\mu_{T}$ is modeled as:

$$
\mu_{T}=\bar{\rho}\left(C_{S} \Delta\right)^{2}|\widetilde{\mathbf{S}}|=\bar{\rho}\left(C_{S} \Delta\right)^{2}\left(2 \widetilde{S_{i j}} \widetilde{S_{i j}}\right)^{1 / 2}
$$

Here $C_{S}$ is a model constant (given in table 3.1) and $\Delta$ the filter width (defined in subsection 3.2.1).

The sub grid fluctuations of species mass fraction and enthalpy are modeled using a classic gradient assumption [75]:

$$
\begin{aligned}
\bar{\rho} \overline{\mathbf{u}^{\prime \prime} Y_{k}^{\prime \prime}}=-\bar{\rho} D_{T} \nabla \widetilde{Y_{k}} & =-\frac{\mu_{T}}{S c} \nabla \widetilde{Y_{k}} \\
\bar{\rho} \overline{\mathbf{u}^{\prime \prime} h^{\prime \prime}}=-\bar{\rho} D_{\lambda} \nabla \widetilde{h} & =-\frac{\mu_{T}}{P r} \nabla \widetilde{h}
\end{aligned}
$$

with $S c$ the turbulent Schmidt number, $\operatorname{Pr}$ the turbulent Prandtl number and $\mu_{T}$ the turbulent viscosity, calculated by the turbulence model.

\begin{tabular}{lll}
\hline$C_{S}$ & $P r$ & $S c$ \\
\hline 0.2 & 0.7 & 0.7 \\
\hline
\end{tabular}

Table 3.1: Constants in the turbulence model. The standard values that are implemented in OpenFoam are used [1].

\subsubsection{Combustion model}

As mentioned before, for practical applications it is not feasible to solve equation 3.8 for each individual specie due to the amount of CPU power that would be required. Therefore, the combustion process has to be simplified. Several combustion models for LES have been developed. A detailed description of available LES combustion models for partially premixed flows can be found in Knudsen et al. [50]. A list of well known LES combustion models includes:

- Thickened Flame Models, see for instance Poinsot et al. [15]. These models solve a set of transport equations for a reduced number of chemical species and use Arrhenius rate expressions to describe species' reactions. These transport equations can only be solved, however, when the flame structure is adequately resolved. Resolving realistic flame structures is not affordable in LES, so Thickened Flame Models artificially broaden flame structures in order to ensure convergence of the species equations.

- Transported PDF models, see e.g. Pope et al. [79]. These models attempt to describe chemical reactions by solving for a sub filter Probability Density Function distribution. 
- Flamelet Generated Manifolds (FGM) [94]. This method maps solutions from a precomputed laminar database onto a 3D turbulent flow field. The reactive source term is determined from this database based on a number of control variables that are calculated by the CFD code (e.g. mixture fraction and progress variable).

- Flame Surface Density model (or Turbulent Flame speed Closure) [37]. In FSD models, the combustion process is modeled based on a precomputed laminar flame speed (which is a function of the mixture fraction) and a wrinkling factor, which is a function of the local turbulence. Due to its relatively low cost, this model is very popular.

The combustion model used in the present work is a Flame Surface Density Model, described in detail below.

If one assumes a simple one step reaction:

$$
\text { Fuel }+ \text { Oxidizer } \rightarrow \text { Products }
$$

The transport equation for the $k^{t h}$ species mass fraction $Y_{k}$ (equation 3.8) can conveniently be rewritten as a function of a mixture fraction $f_{T}$ and the reaction progress variable $c$ :

$$
\begin{aligned}
Y_{k} & =Y_{k}\left(f_{T}, c\right) \\
f_{T} & =\frac{Y_{k}-Y_{k O}}{Y_{k F}-Y_{k O}} \\
c & =\frac{Y_{k}-Y_{k, \text { unburnt }}}{Y_{k, \text { burnt }}-Y_{k, \text { unburnt }}}
\end{aligned}
$$
flow.

with $Y_{k O}$ the mass fraction in the pure oxidizer flow and $Y_{k F}$ in the pure fuel

The mixture fraction $f_{T}$ indicates the mass ratio between fuel and oxidizer. The progress variable $c$ is a non-dimensional parameter that acts as a measure of the degree of 'reactedness' of the mixture, varying from zero in the unburnt mixture to one in the fully burnt mixture. The introduction of the mixture fraction and progress variable simplifies equation 3.8 to:

$$
\begin{aligned}
\frac{\partial\left(\bar{\rho} \widetilde{f_{T}}\right)}{\partial t}+\nabla \cdot\left(\bar{\rho} \widetilde{\mathbf{u}} \widetilde{f_{T}}\right) & =\nabla \cdot\left(\left(\bar{\rho} \bar{D}+\frac{\mu_{T}}{S c}\right) \nabla \widetilde{f_{T}}\right) \\
\frac{\partial(\bar{\rho} \widetilde{c})}{\partial t}+\nabla \cdot(\bar{\rho} \widetilde{\mathbf{u}} \widetilde{c}) & =\nabla \cdot\left(\left(\bar{\rho} \bar{D}+\frac{\mu_{T}}{S c}\right) \nabla \widetilde{c}\right)+\widetilde{\dot{w}_{R}}
\end{aligned}
$$

Note that the gradient assumption for the sub grid fluctuations (see equation 3.16) has already been used here.

The term that has not yet been discussed is the chemical source term $\widetilde{\dot{w}_{R}}$ in the transport equation for the reaction progress variable. In Flame Surface Density models 
(also called Turbulent Flame speed Closure), the concept of the laminar flame speed $S_{L}$ is extended to its turbulent equivalent $S_{T}$. In this model the chemical source term is defined as [23]:

$$
\widetilde{\dot{w}_{R}}=\overline{\rho_{u}} S_{T}|\nabla \widetilde{c}|=\overline{\rho_{u}} S_{L}^{0} \Sigma \Xi|\nabla \widetilde{c}|
$$

Here $\rho_{u}$ is the density of the unburnt mixture. To obtain the unburnt gas properties, under the hypothesis that $\widetilde{c}=0$, a separate enthalpy equation is solved (equation 3.25). This enthalpy equation yields the local hypothetical temperature and density for unburnt gas.

$$
\frac{\partial\left(\bar{\rho} \widetilde{h_{u}}\right)}{\partial t}+\nabla \cdot\left(\bar{\rho} \widetilde{\mathbf{u}} \widetilde{h_{u}}\right)=\frac{D \bar{p}}{D t} \frac{\bar{\rho}}{\overline{\rho_{u}}}+\nabla \cdot\left(\frac{\mu_{T}}{P r} \nabla \widetilde{h_{u}}\right)
$$

In equation 3.24, $S_{L}^{0}=S_{L}^{0}\left(f_{T}\right)$ is the unstrained laminar flame speed. The unstrained laminar flame speed is obtained using a one-dimensional premixed flame computation (in this case using Cantera) as a function of the mixture fraction. With this computation, a look-up table is created, which is used by the CFD code to calculate $S_{L}^{0}$ from $f_{T}$.

The factor $\Sigma$ is the ratio between the turbulent and laminar flame speed: the sub grid scale wrinkling factor. Several models (e.g. Bradley [10], Gülder [35], Zimont [97]) have been proposed. The model that is used in the current work is the one developed by Bradley [10]. The correlation is based on a broad range of experimental data.

$$
\Sigma=\frac{S_{T}}{S_{L}}=1+\frac{A_{F S D}}{L e}\left(\frac{u_{S G S}^{\prime \prime} l_{T}}{S_{L} l_{f}}\right)^{0.5}=1+\frac{A_{F S D}}{L e}\left(\frac{u_{S G S}^{\prime \prime} l_{T}}{\alpha_{u}}\right)^{0.5}
$$

The value of the constant $A_{F S D}$ is $0.5, L e$ is the Lewis number and $u_{S G S}^{\prime \prime}, l_{T}$ and $\alpha_{u}$ are the sub grid velocity, length scale and thermal diffusivity respectively.

The parameter $\Xi$ takes into account that the flame speed could be reduced (quenching effect) when the flame is subjected to large strain rates and/or heat losses. In order to model these effects, the following model, which is based on the model of Tay et al. [13], was used:

$$
\Xi=\frac{S_{L}}{S_{L}^{0}}=\left(1-M a^{*} \cdot \ln (1+K a) \exp (-K a \cdot \chi)\right)
$$


with $S_{L}$ the strained laminar flame speed and:

$$
\begin{aligned}
K a & =\frac{\alpha_{u} \kappa}{S_{L}^{0^{2}}} \\
\beta & =\frac{T_{a d}-T_{\text {loss }}-T_{u}}{T_{a d}-T_{u}} \\
\chi & =\frac{Z e}{2} \frac{1-\beta}{\beta^{3}} \\
Z e & =\frac{E_{a}}{2 T_{a d}^{2}}\left(T_{a d}-T_{u}\right)
\end{aligned}
$$

In this model, $\kappa$ is the strain rate and $T_{u}$ and $T_{a d}$ are the temperatures of the unburnt gas and the adiabatic flame temperature respectively. $M a^{*}$ is a model parameter which is obtained by fitting the outlined model to results from a 1D counter flow simulation for a range of heat loss numbers $\beta$ and Karlovitz numbers $K a$. The exact procedure is described by Tay et al. [13]. The other parameters are dimensionless numbers and are defined above. This model improves the calculation of the

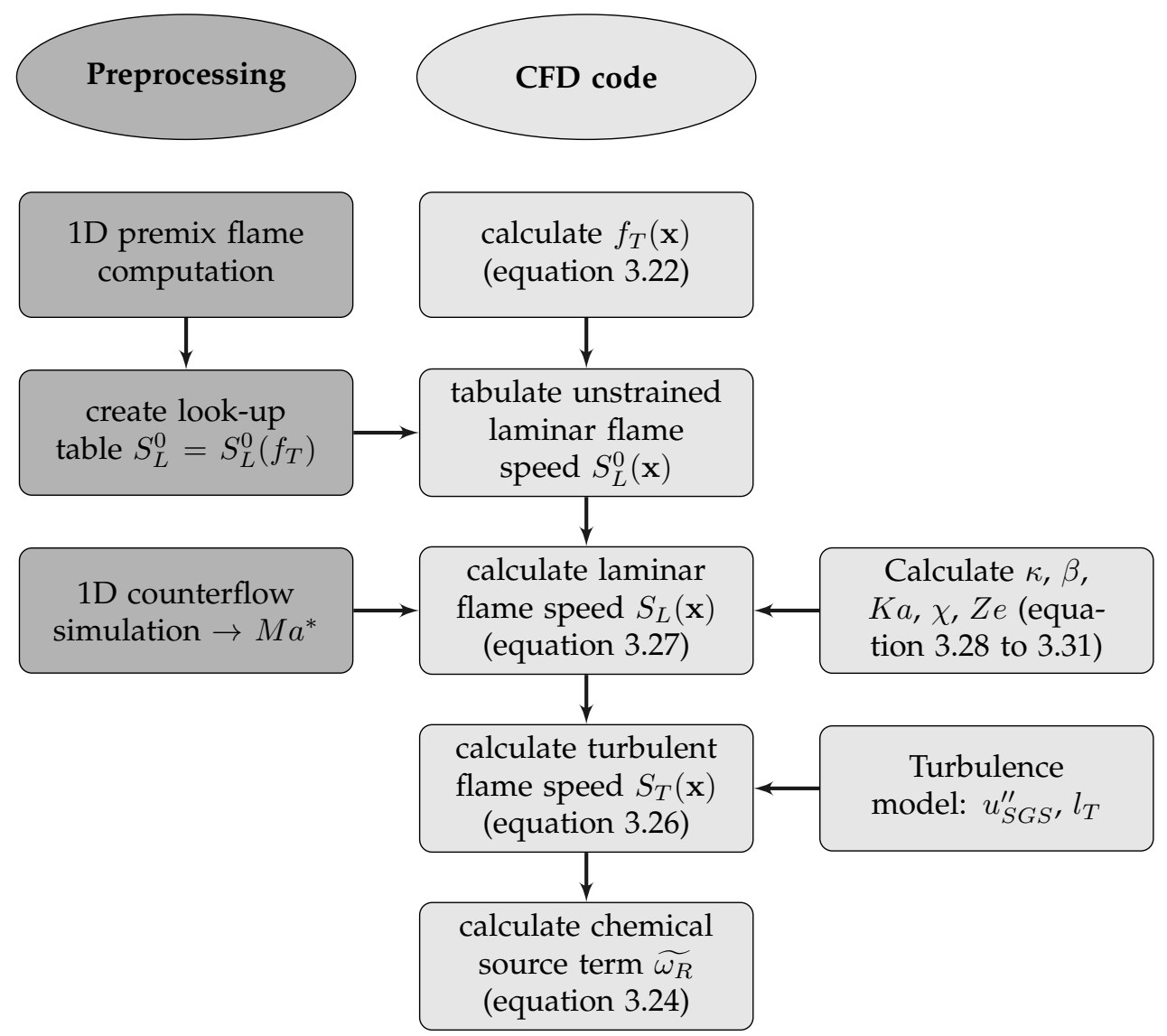

Figure 3.3: Flowchart of the combustion model. 
turbulent flame speed in regions of large shear and regions close to walls where nonadiabatic conditions prevail. The implementation of the combustion and quenching model in OpenFOAM was validated by di Domenico et al. [23].

The combustion model and the flow of information are summarized in figure 3.3.

\subsubsection{Final equation set implemented in the reacting LES code}

The set of transport equations that is implemented in the reacting LES code used in this work is derived by inserting the equations for the combustion model (3.22 to 3.25 ) and for the turbulence model (equations 3.14 to 3.17) into equation 3.6 to 3.13. The final result is summarized below:

Mass:

$$
\frac{\partial \bar{\rho}}{\partial t}+\nabla \cdot(\bar{\rho} \widetilde{\mathbf{u}})=0
$$

Momentum:

$$
\frac{\partial(\bar{\rho} \widetilde{\mathbf{u}})}{\partial t}+\nabla \cdot(\bar{\rho} \widetilde{\mathbf{u}} \otimes \widetilde{\mathbf{u}})=-\nabla \bar{p}+\nabla \cdot \overline{\bar{\tau}}-\nabla \cdot\left(\bar{\rho} \overline{\mathbf{u}^{\prime \prime} \mathbf{u}^{\prime \prime}}\right)
$$

with $\bar{\rho} \overline{\mathbf{u}^{\prime \prime} \mathbf{u}^{\prime \prime}}$ calculated from equation 3.14 and 3.15 and $\overline{\bar{\tau}}$ from equation 3.12.

Mixture fraction:

$$
\frac{\partial\left(\bar{\rho} \widetilde{f_{T}}\right)}{\partial t}+\nabla \cdot\left(\bar{\rho} \widetilde{\mathbf{u}} \widetilde{f_{T}}\right)=\nabla \cdot\left(\left(\bar{\rho} \bar{D}+\frac{\mu_{T}}{S c}\right) \nabla \widetilde{f_{T}}\right)
$$

Reaction progress variable:

$$
\begin{aligned}
\frac{\partial(\bar{\rho} \widetilde{c})}{\partial t}+\nabla \cdot(\bar{\rho} \widetilde{\mathbf{u}} \widetilde{c}) & =\nabla \cdot\left(\left(\bar{\rho} \bar{D}+\frac{\mu_{T}}{S c}\right) \nabla \widetilde{c}\right)+\widetilde{\dot{w}_{R}} \\
& =\nabla \cdot\left(\left(\bar{\rho} \bar{D}+\frac{\mu_{T}}{S c}\right) \nabla \widetilde{c}\right)+\overline{\rho_{u}} S_{T}|\nabla \widetilde{c}|
\end{aligned}
$$

Unburnt enthalpy:

$$
\frac{\partial\left(\bar{\rho} \widetilde{h_{u}}\right)}{\partial t}+\nabla \cdot\left(\bar{\rho} \widetilde{\mathbf{u}} \widetilde{h_{u}}\right)=\frac{D \bar{p}}{D t} \frac{\bar{\rho}}{\overline{\rho_{u}}}+\nabla \cdot\left(\frac{\mu_{T}}{P r} \nabla \widetilde{h_{u}}\right)
$$

Total enthalpy:

$$
\frac{\partial(\bar{\rho} \widetilde{h})}{\partial t}+\nabla \cdot(\bar{\rho} \widetilde{\mathbf{u}} \widetilde{h})=\frac{D \bar{p}}{D t}+\nabla \cdot\left(\frac{\mu_{T}}{P r} \nabla \widetilde{h}\right)
$$




\subsubsection{Numerical simulation}

The transport equations derived above are discretized in time and space and solved using the PISO algorithm (Pressure Implicit with Splitting of Operators). The PISO algorithm is a semi-implicit method. The method first solves the Momentum equation in order to obtain a velocity field. Following, the transport equations for mixture fraction, progress variable, enthalpy and pressure are solved in a so called PISO loop. Each equation is solved sequentially, but multiple times (usually 2 or 3 loops are made) within one time step. More information about the PISO algorithm can by found in the PhD work of Jasak [46].

OpenFOAM offers several spatial and temporal discretization schemes, the most important ones being the $1^{\text {st }}$ and $2^{\text {nd }}$ order Euler schemes and the Crank Nicolson scheme. The Crank Nicolson scheme is a $2^{\text {nd }}$ order scheme, with the difference that the solution is blended with a small percentage of $1^{\text {st }}$ order solution. This makes the scheme more robust than the $2^{\text {nd }}$ order Euler scheme. Discretization of $1^{\text {st }}$ order is known to damp unsteady phenomena [75]. For high quality LES simulations, at least $2^{\text {nd }}$ order discretization is required. All results presented in this dissertation have been obtained using the $2^{\text {nd }}$ order Crank Nicolson scheme with $20 \%$ first order blending.

Two important numbers that determine the time step that can be used in the simulation are the Courant number $(\mathrm{Co})$, given by equation 3.38, and the CFL-number $(C F L)$, given by equation 3.39. The Courant number is based on the convective velocity, whereas the CFL-number is based on the speed of sound. For stable numerical solution using the PISO algorithm, it is required that $C o<0.3$ everywhere in the numerical domain. In fully explicit codes, it is required that $C F L<0.7$, but this restriction on the time step does not apply to the PISO algorithm. However, as will be shown in subsection 3.4.1, the CFL number does have an impact on the dissipation and dispersion of acoustic waves. It will be shown in this subsection that the value of the CFL number that needs to be chosen depends on the frequency range of the unsteady phenomena that should be resolved.

$$
\begin{aligned}
C o & =\frac{|\mathbf{u}| \Delta t}{\Delta x} \\
C F L & =\frac{(|\mathbf{u}|+c) \Delta t}{\Delta x}
\end{aligned}
$$

\subsection{CFD simulation of thermo-acoustic systems}

As mentioned in the introduction of this chapter (section 3.1), first a stable and converged CFD solution should be obtained, without excitation of the boundaries. This simulation is used as an initial solution for the simulation with excitation. After convergence, the boundary conditions are then perturbed and the acoustic quantities of interest (pressure, velocity, mixture fraction and heat release) are sampled on specific sampling planes. Finally, time series of these acoustic quantities are postprocessed in order to get the required transfer function. 
The specific features of the boundary conditions, sampling planes and the postprocessing are discussed in this section.

\subsubsection{Boundary conditions}

The standard boundary conditions in CFD codes for pressure and velocity are fully reflective. When a fixed velocity at the inlet is applied, the acoustic fluctuation $u^{\prime}=$ 0 . This would correspond to a acoustically closed boundary condition. A constant pressure at the outlet $\left(p^{\prime}=0\right)$ would correspond to an acoustically open boundary condition.

Yuen et al. [96] investigated the influence of the boundary reflection coefficient on the transfer function identification of acoustic systems. It was found that the numerical accuracy of the identification strongly increases with decreasing reflection coefficient. A reason for this is that resonance frequencies of the system may be trigged when reflecting boundary conditions are used. This causes large pressure peaks at certain frequencies, while the pressure at non resonance frequencies is much lower.

To avoid acoustic reflections, the simulations with excitation presented in this dissertation have been performed with non reflecting boundary conditions. The SIEMENS CFD code has non reflective boundary conditions implemented based on the LODI approach (Local One-Dimensional Inviscid). It should be noticed that these boundary conditions are not available in the standard OpenFOAM version. More information about the LODI approach is given by Polifke [78] or Poinsot [75]. In Appendix $C$ the specific equations solved in the LODI approach can be found. With these boundary conditions, disturbances are separated into waves traveling into the domain and out of the domain.

In practice fully non reflective boundary conditions are very hard to implement, otherwise information about the far field properties (e.g. the mean inlet velocity and mean outlet pressure) cannot propagate into the system. Therefore a small percentage of reflection is always required. In the OpenFOAM implementation (see Appendix C), the LODI relations are relaxed by the time scalar $\tau$. The boundary condition now behaves like a low pass filter, with its cut-off frequency depending on the magnitude of $\tau$. In figure 3.4, the reflection coefficient as a function of frequency and the time scalar is shown.

The minimal value for $\tau$ that should be chosen depends on the lowest Eigenfrequency of the geometry. One could think that an arbitrary large value of $\tau$ is ideal, because it assures low reflection even for very small frequencies. However, the larger the value of $\tau$, the more difficult it is for far stream information (mean inlet velocity and mean outlet pressure) to propagate into the system. A too large value of $\tau$ may cause the mean velocity and pressure to drift away from the desired mean values [78]. On the other hand, if $\tau$ is too small, the simulation may become unstable due to the reflection of small frequencies. Therefore, the value of $\tau$ should be optimized for each simulation, such that $f \cdot \tau=\mathcal{O}\left(10^{1}\right)$.

To identify the acoustic response of a given configuration, the (inlet) boundary condition should be perturbed. Several options are available to do so, e.g. white noise, pulsed excitation, harmonic excitation. In the current implementation, white 


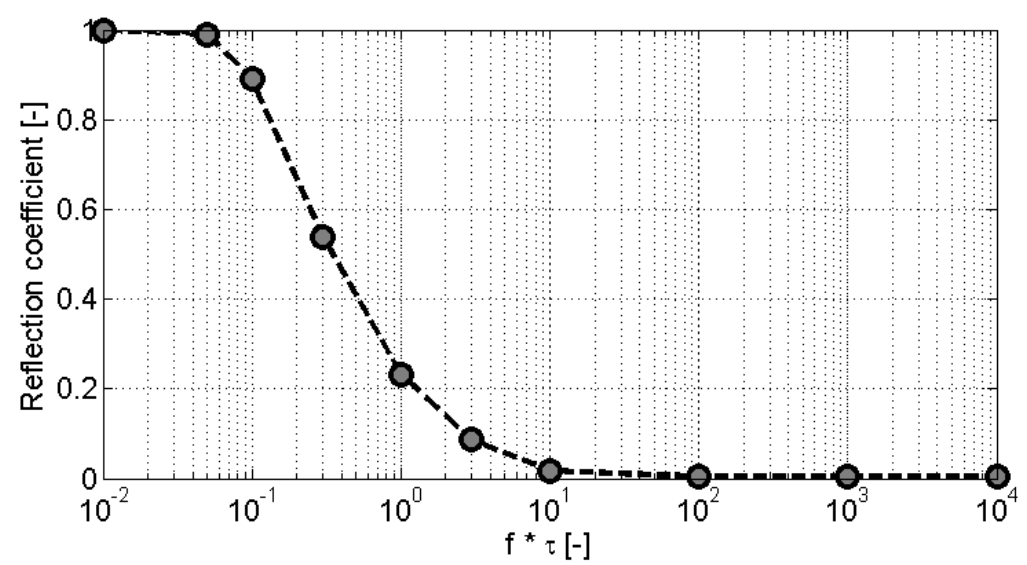

Figure 3.4: Reflection coefficient as a function of frequency and relaxation parameter $\tau$

noise can only be applied in case reflective boundary conditions are used, because the non reflective inlet boundary condition requires the perturbation to be continuously differentiable. For this reason, a superposition of harmonic excitations (sinus overlay) is used in the code. This signal contains $N$ frequencies between $f_{\text {low }}$ and $f_{\max }$. The lower frequency $f_{\text {low }}$ should be chosen such that it is in the non reflective range (see figure 3.4).

In case of a velocity inlet boundary condition, the perturbation can be written as:

$$
\mathbf{u}_{\infty}(t)=\mathbf{u}_{\infty}^{0}\left(1+A \sum_{i=1}^{N} \sin 2 \pi f_{i} t\right)
$$

where $\mathbf{u}_{\infty}^{0}$ is the mean far stream velocity and $A$ is the amplitude of the perturbation. In case $N=1$, the signal is a pure single harmonic wave. The subscript $\infty$ indicates that the velocity at the boundary condition is not directly prescribed, but rather the far stream information (see appendix C).

\subsubsection{Sampling of acoustic quantities}

In order to derive an acoustic transfer function from the CFD simulation, e.g. a Flame Describing Function, time series of the area averaged velocity, pressure, mixture fraction have to be extracted at specific reference planes. The heat release is integrated over the entire volume where heat release takes place. It was suggested by Polifke et al. $[32,76]$ that the following averaging procedure should be applied for optimal results: 


$$
\begin{aligned}
u_{\text {plane }}(t) & =\frac{\int \widetilde{\rho}(\mathbf{x}, t) \widetilde{\mathbf{u}}(\mathbf{x}, t) \cdot \mathbf{n} d A}{\int \widetilde{\rho}(\mathbf{x}, t) d A} \\
p_{\text {plane }}(t) & =\frac{\int \bar{p}(\mathbf{x}, t) d A}{\int d A} \\
f_{T, p l a n e}(t) & =\frac{\int \widetilde{\rho}(\mathbf{x}, t) \widetilde{f_{T}}(\mathbf{x}, t) d A}{\int \widetilde{\rho}(\mathbf{x}, t) d A} \\
Q(t) & =\int \widetilde{q}(\mathbf{x}, t) d V=\int H_{f} \widetilde{\tilde{w}_{R}}(\mathbf{x}, t) d V
\end{aligned}
$$

This means that the velocity and mixture fraction are density weighted, which is important for applications with large density gradients over the sampling plane. In figure 3.5, a sketch is shown of a simple burner configuration with an air and a fuel inlet. Typical positions of reference planes on which velocity, pressure and mixture fraction are sampled are indicated in the figure, which is usually upstream of the flame. For partially premixed systems, which have mixture fraction fluctuations, separate sampling planes should be placed at the inlet of the combustion chamber and at location of the fuel injection.

In equation 3.44, $Q$ is the volume integrated heat release rate, integrated over the combustion chamber. The parameter $H_{f}$ (unit J/ $\mathrm{kg}$ fuel) is the specific enthalpy of reaction.

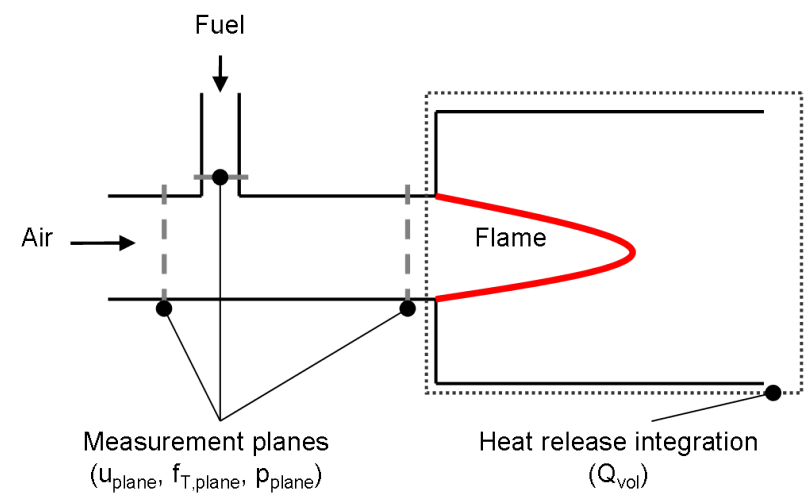

Figure 3.5: Typical location of reference planes.

\subsubsection{Postprocessing of thermo-acoustic response}

After performing the transient CFD simulation with excitation of the boundary conditions, the sampled time series are postprocessed using Matlab in order to get the transfer functions. First, the quantities are split into their mean and fluctuating parts: 


$$
\begin{array}{cccc}
u^{\prime}(t)=u_{\text {plane }}(t)-u_{0} & \text { with } & u_{0}=\frac{1}{T} \int_{0}^{T} u_{\text {plane }}(t) d t \\
p^{\prime}(t)=p_{\text {plane }}(t)-p_{0} & \text { with } & p_{0}=\frac{1}{T} \int_{0}^{T} p_{\text {plane }}(t) d t \\
f_{T}^{\prime}(t)=f_{T, p l a n e}(t)-f_{T, 0} & \text { with } & f_{T, 0}=\frac{1}{T} \int_{0}^{T} f_{T, p l a n e}(t) d t \\
Q^{\prime}(t)=Q(t)-Q_{0} & \text { with } & Q_{0}=\frac{1}{T} \int_{0}^{T} Q(t) d t
\end{array}
$$

were $T$ represents the total simulation time.

As discussed in section 3.3.1, the excitation signal can be either a single harmonic wave or a broadband signal. Most simulations that will be presented in this dissertation have been carried out using single frequency excitation. In this case, the transfer function between two properties can be determined using Fast-Fourier transformation (FFT). The discrete version of the FFT assumes that the time record is exactly repeated throughout all time, and thus that the time record is periodic at the ends. In case this criterion is not met (which is generally the case for time series of finite length) and the FFT would be directly applied, energy from a given frequency could leak over the adjacent frequencies in the Fourier transform (spectral leakage). In order to prevent this spectral leakage, windowing functions have to be used. In this work a Hanning window is applied:

$$
\begin{aligned}
H(t) & =0.5(1-\cos (2 \pi t / T)) \\
\widehat{H}(t) & =\frac{H(t)}{\frac{1}{T} \int_{0}^{T} H(t) d t}
\end{aligned}
$$

The time series of equations 3.45 to 3.48 are directly multiplied with the normalized window $\widehat{H}$. The window can be seen as a weighting function, it is zero at the ends of the signal and has the maximum value in the middle. Principally, for single frequency harmonic excitation, a window is not required when the time series contain an integer number of cycles (ensuring periodicity at the boundaries). Nonetheless, also in this case the Hanning window is used.

Equations 3.45 to 3.48 can now be Fourier-transformed. In case of the acoustic velocity, the FFT is calculated as: *

$$
\hat{u}(\omega)=\int_{0}^{T} u^{\prime}(t) \widehat{H}(t) e^{-i \omega t} d t
$$

The Fourier transformation for the other fluctuating quantities is calculated in the same way.

\footnotetext{
*Equations 3.51, 3.53 and 3.54 are calculated with Matlab in discrete form. The discrete equivalents of the FFT, auto-correlation and cross-correlation can be found in the Matlab Reference Guide [65].
} 
After applying the Fourier transformation, the transfer function can be determined. For a Flame Describing Function, the signal is the velocity fluctuation upstream of the flame and the response is the fluctuating heat release rate. The FDF can then be determined as:

$$
\mathcal{F}\left(\omega,\left|u^{\prime}\right|\right)=\frac{\hat{Q}(\omega) / Q_{0}}{\hat{u}(\omega) / u_{0}}
$$

An alternate way of determining transfer functions (see e.g. van Kampen [47]) consists of calculating the auto-correlation vector $R_{u u}$ and cross-correlation vector $R_{u Q}$ :

$$
\begin{aligned}
R_{u u}(t) & =\int_{0}^{T-t} \frac{u^{\prime}(\tau)}{u_{0}} \frac{u^{\prime}(t+\tau)}{u_{0}} d \tau \\
R_{u Q}(t) & =\int_{0}^{T-t} \frac{u^{\prime}(\tau)}{u_{0}} \frac{Q^{\prime}(t+\tau)}{Q_{0}} d \tau
\end{aligned}
$$

In this case the FDF is determined as:

$$
\mathcal{F}\left(\omega,\left|u^{\prime}\right|\right)=\frac{\hat{R}_{u Q}(\omega)}{\hat{R}_{u u}(\omega)}
$$

Here $\hat{R}_{u Q}(\omega)$ is the Fourier transformed equivalent of $R_{u Q}(t)$. When this version is used for evaluating the transfer function, the influence of noise in the response signal on the transfer function can significantly be reduced [47]. Using such an approach is especially important for the postprocessing of noisy signals (e.g. pressure transducer measurements). However, during the postprocessing of the CFD simulations that will be presented in Chapter 4 and 5 of this dissertation, negligible differences between the results obtained via equation 3.52 and equation 3.55 were found. Therefore equation 3.52 is used throughout.

In case broadband excitation is used, different post processing methods have to be used, such as the Wiener Hopf Transformation [76]. The Wiener Hopf Transformation can only be used for small (linear) excitations $\left(u^{\prime} / u_{0} \leq 0.1\right)$. This means, it cannot be used for determining the Flame Describing Function, since excitation amplitudes $u^{\prime} / u_{0}>0.1$ are required. A description of the Wiener Hopf transformation can be found in Appendix E.

Ideally the Flame Describing Function for large perturbation amplitudes is determined using broadband excitation as well. However post processing methods for high amplitude broadband excitation are not available. In Appendix G however, it is investigated if the FDF can be determined using a superposition of harmonic waves.

\subsection{Verification for thermo-acoustic applications}

Before applying the CFD code to complex reacting flows and determining the Flame Describing Function, the general performance of the code for thermo-acoustic applications has first been verified using simple, non-reacting test cases. These cases will be presented in the following subsections: 
- Propagation of a 1D acoustic wave (subsection 3.4.1). This case is important to test the dissipation and dispersion characteristics of the code. The gain of the Flame Describing Function is expected to decrease for larger excitation amplitudes (the flame saturates). This saturation should be caused by physical processes in the simulation and not by numerical damping. The numerical damping of the code is tested in a non-reacting environment.

- High amplitude boundary excitation (subsection 3.4.2). In order to obtain the Flame Describing Function, large excitation amplitudes are required. The excitation amplitude at the inlet may even be larger than the mean flow. This kind of boundary condition should therefore be tested before application to complex geometries.

- Transfer function of an area discontinuity (Appendix D). An area discontinuity is a relevant test case because it is a typical acoustic element present in most burner configurations (see for instance figure 3.5).

\subsubsection{Propagation of a 1D acoustic wave}

In order to accurately resolve a Flame Describing Function, small numerical dissipation and dispersion are required. This guarantees that the expected saturation of the flame at higher excitation amplitudes is caused by physical effects, not by numerical damping. In this section, the dissipation and dispersion characteristics of the code are verified.

In figure 3.6, dissipation and dispersion are defined. The figure shows a sketch with an exact solution and a numerical solution of an acoustic wave. Dissipation is the difference in amplitude of the acoustic pressure between the exact and numerical solution. Dispersion on the other hand stands for the phase error.

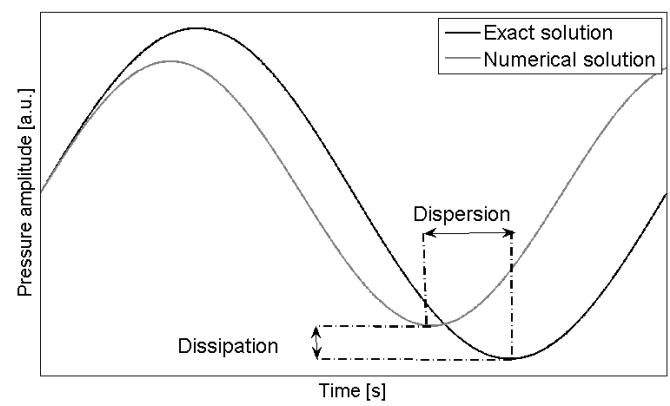

Figure 3.6: Definition of dissipation and dispersion.

\section{CFD Approach}

To investigate the dispersion and dissipation of the code, a one-dimensional tube of 2 meters long has been simulated. The grid consisted of 500 hexahedral cells, 
resulting in $\Delta x=0.004 \mathrm{~m}$. At the inlet, a mean velocity of $u_{0}=10 \mathrm{~m} / \mathrm{s}$ and a mean temperature of $493 \mathrm{~K}$ were imposed. A non-reflecting outlet boundary condition with a mean pressure of 1 bar was used. An acoustic wave with amplitude $u^{\prime}=1$ $\mathrm{m} / \mathrm{s}$ has been superimposed on the inlet boundary condition. The frequency of the acoustic wave has been varied, as well as the CFL number of the simulation.

\section{Results}

In figure 3.7, the velocity fluctuation $u^{\prime}$ as a function of the axial coordinate can be seen after $8 \mathrm{~ms}$ simulation. The axial coordinate is normalized by the acoustic wave number $\lambda=c / f$ and the results are shown as function of the CFL-number and the number of cells per wave length $\left(n_{\text {cells }}=\lambda / \Delta x\right)$. Results are shown for 10, 20, 30, 50, 100 and 200 cells per wave length and for four different CFL-numbers (CFL $=0.35,0.70,2.1$ and 4.9). As can be observed from the figures (and as expected), numerical damping increases when the CFL-number is increased and when less cells per wave length are used. To compare the dissipation and dispersion of the different simulation, the following definitions are used:

$$
\begin{aligned}
\text { Dissipation } & =\frac{\max \left(u^{\prime}(x=[0: \lambda])\right)-\max \left(u^{\prime}(x=[\lambda: 2 \lambda])\right)}{\max \left(u^{\prime}(x=[0: \lambda])\right)} \\
\text { Dispersion } & =\frac{x\left(\max \left(u^{\prime}(x=[\lambda: 2 \lambda])\right)-x\left(\max \left(u^{\prime}(x=[0: \lambda])\right)\right)-\lambda\right.}{\lambda}
\end{aligned}
$$

In figure 3.8, the dissipation and dispersion according to equation 3.56 and 3.57 are shown. A typical wave length for a quarter wave longitudinal mode in a combustion system would be in the order of $\lambda=c / f=750 / 250=3 \mathrm{~m}$. With a grid size of $0.003 \mathrm{~m}$, which would be a course mesh, this results in 1000 cells per wave length. Extrapolating the results of figure 3.8, it can be concluded that dissipation is negligible for the frequencies of interest, even for CFL numbers larger than 0.7.

The dispersion is only shown for $\mathrm{CFL}=4.9$. For smaller $\mathrm{CFL}$ numbers, the dispersion was too small to be accurately determined. As can be seen from the figure, also dispersion is negligible for the frequencies of interest in practical applications.

\subsubsection{High amplitude boundary excitation}

In order to obtain the Flame Describing Function, large excitation amplitudes are required. Therefore, the acoustic velocity $u^{\prime}$ at the burner exit, upstream of the flame, needs to be in the order of the mean flow velocity $\left(u^{\prime} \approx u_{0}\right)$. The required excitation amplitude at the inlet boundary condition, which is located upstream of the burner, could even be larger. For the swirl stabilized combustor presented in the next chapter, excitation amplitudes of up to 3 times the mean flow velocity were required. The reason is that due to the many area discontinuities present in a typical burner, part of the acoustic wave does not reach the flame, but reflects back to the non-reflecting inlet and leaves the domain. 

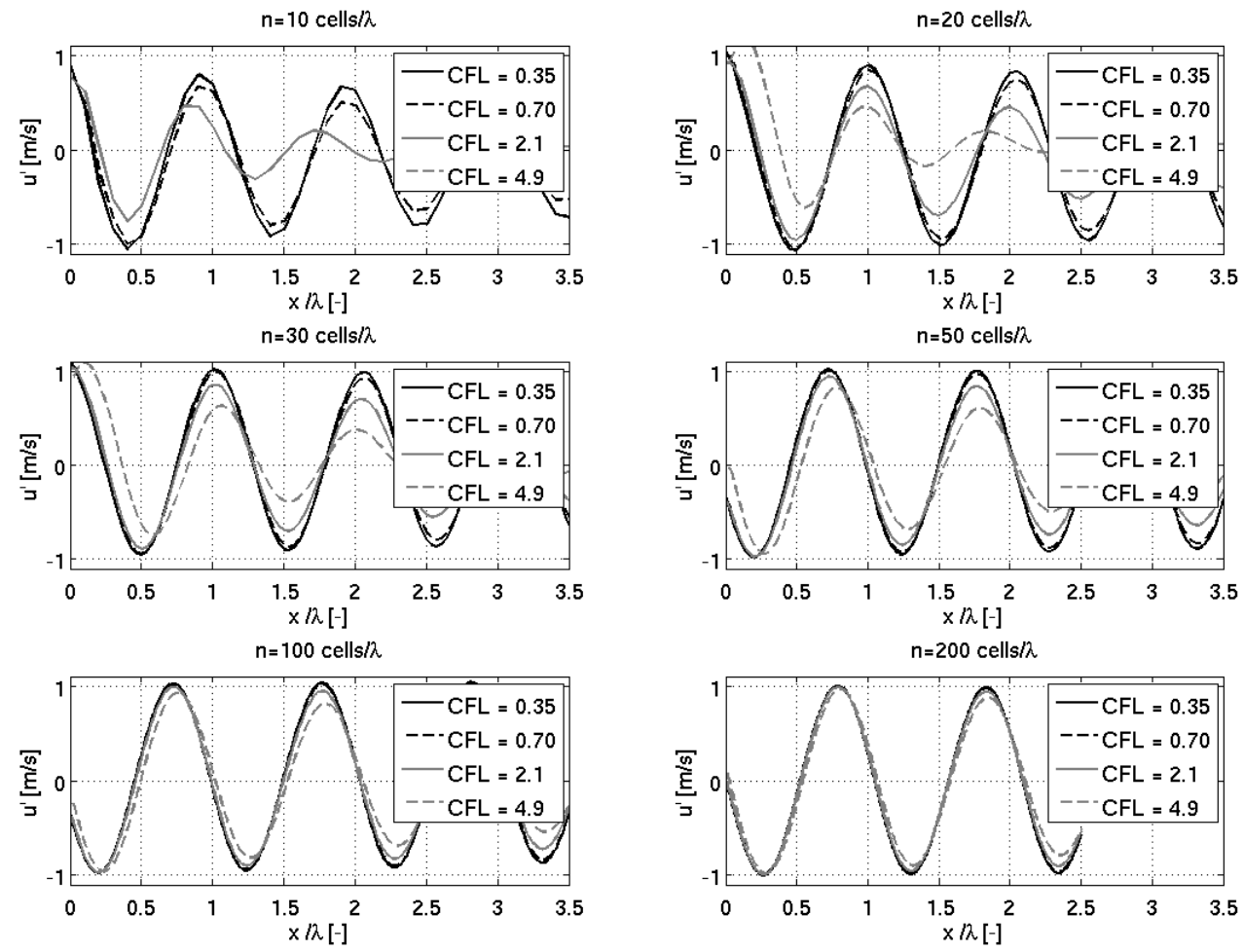

Figure 3.7: Acoustic velocity distribution in the 1D tube at arbitrary time $t$, as function of CFL number and cells per wave length $\left(n_{\text {cell }}=\lambda / \Delta x\right)$. The frequency of the acoustic velocity excitation at the inlet is $f=c / \lambda$ and the boundary conditions are non-reflecting.
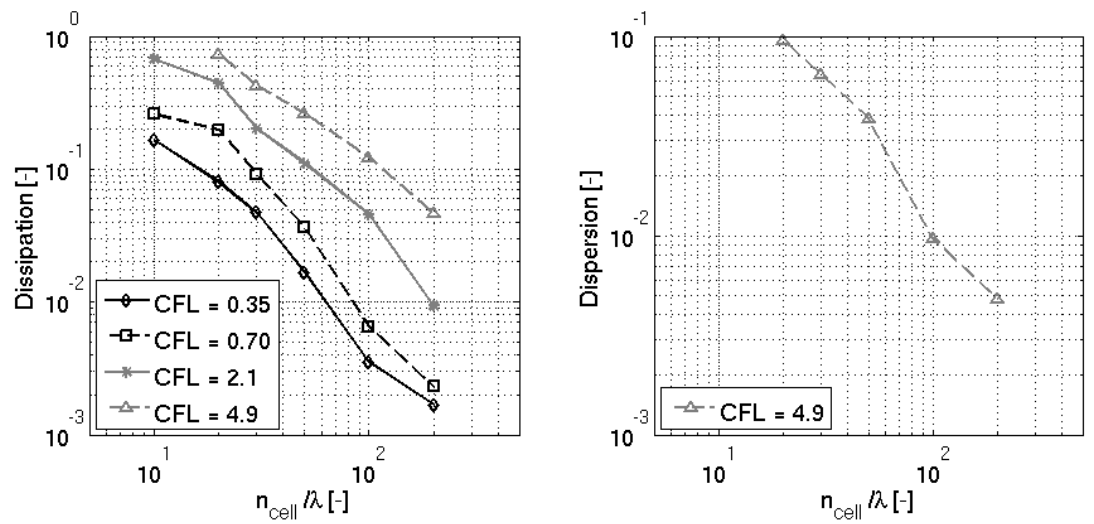

Figure 3.8: Dispersion and dissipation of acoustic waves, as function of CFL number and cells per wave length $\left(n_{\text {cell }} / \lambda\right)$. 
This means that during a part of the acoustic cycle, the net velocity $\left(u^{\prime}+u_{0}\right)$ becomes negative, resulting in outflow through the boundary. The question to be asked here is: Does the non-reflecting inlet boundary condition allow this?

\section{CFD Approach}

To investigate this question, the problem described above has been simulated in an one-dimensional tube of 2 meters long. The grid consisted of 1000 hexahedral cells. At the inlet, a mean velocity of $u_{0}=3.56 \mathrm{~m} / \mathrm{s}$ and a mean temperature of $493 \mathrm{~K}$ were imposed. A non-reflecting outlet boundary condition with mean pressure 1 bar was used. Acoustic waves with different excitation amplitudes $\left(u^{\prime} / u_{0}=0.45\right.$, $u^{\prime} / u_{0}=1.80$ and $u^{\prime} / u_{0}=2.90$ ) have been superimposed on the mean flow. The frequency $f$ of the excitation was $115 \mathrm{~Hz}$ and $258 \mathrm{~Hz}$ and the time scalar was initially set to $\tau=0.03 \mathrm{~s}$, which, according to figure 3.4 , leads to negligible reflection for frequencies of $115 \mathrm{~Hz}$ and higher.

\section{Results}

In figure 3.9, the velocity fluctuation $u^{\prime}$ at a probe in the middle of the tube (located at $1 \mathrm{~m}$ ) can be seen. The velocity has been normalized by the initial mean velocity $u_{0}$. The dashed black line represents the target range (minimum and maximum values of the velocity fluctuation). The gray line represents the actual minima and maxima. Ideally these lines should overlap. This is however only the case for $\left|u^{\prime} / u_{0}\right|_{\text {inlet }}=0.45$ (left column of the figure). For the higher excitation amplitudes, the velocity is not centered around $u^{\prime} / u_{0}=0$, but drifts away. Since the velocity has been normalized by the initial mean velocity, this means that the mean velocity rises during the simulation. After approximately $0.05 \mathrm{~s}$, which is in the order of magnitude of the time scalar, the velocity minima and maxima converge to a constant value.

This drifting effect for large excitation amplitudes is unwanted and seems to be influenced by the time scalar. Therefore, the simulation with $\left|u^{\prime} / u_{0}\right|_{\text {inlet }}=2.95$ at a frequency of $115 \mathrm{~Hz}$ has been repeated for a range of time scalars. This is shown in figure 3.10. It can be seen that when a smaller value of the time scalar is used ( $\tau=$ $0.006 \mathrm{~s}$ ), the velocity drifts away even faster, where as for $\tau=0.15 \mathrm{~s}$, the velocity drifts away at a much slower rate. For $\tau=1 \mathrm{~s}$, which is an order of magnitude larger than the duration of the simulation $\left(t_{\text {sim }}=0.1 \mathrm{~s}\right)$, no drifting can be observed anymore. From this it can be concluded that drifting can be prevented when the time scalar is chosen at least an order of magnitude larger than the length of the simulation.

\section{Discussion}

The statement that very large values of $\tau$ should be chosen in order to prevent the mean velocity from drifting away from the target mean velocity seems to contradict the discussion about the time scalar in section 3.3.1. In this section it was stated that a too large value of $\tau$ may cause the mean velocity and pressure to drift away from the desired mean values. This contradiction can be explained as follows: 

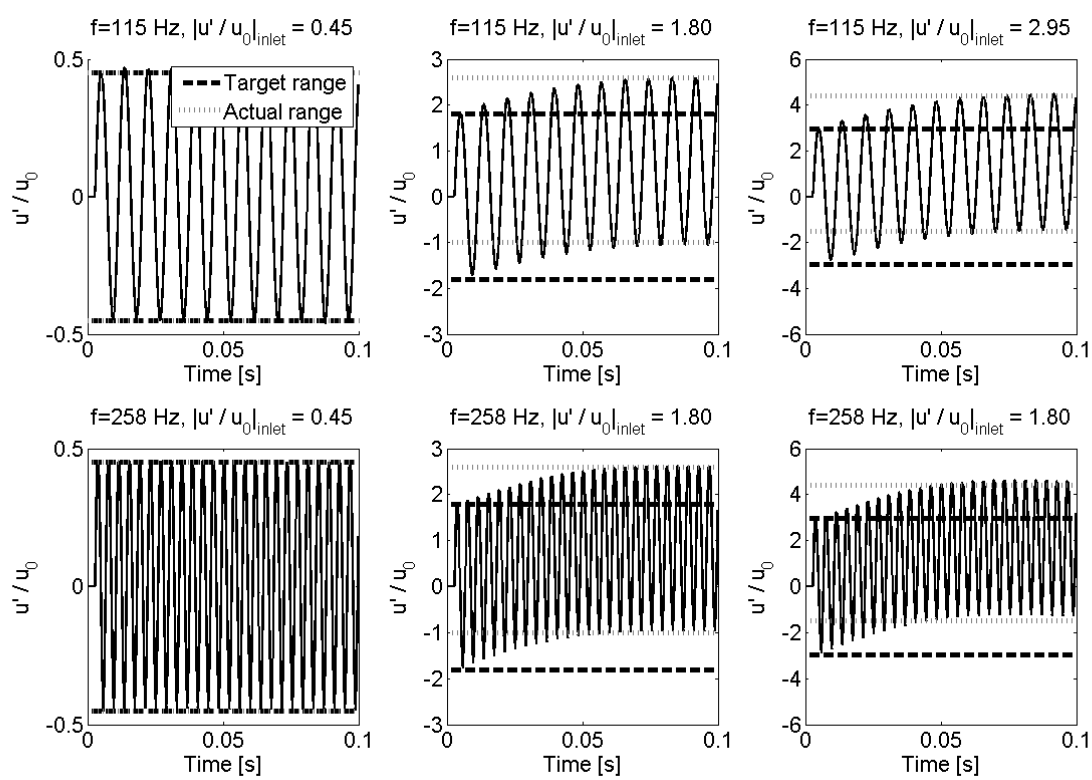

Figure 3.9: Drifting of the velocity, as function of frequency $f$ and excitation amplitude $\left|u^{\prime} / u_{0}\right|_{\text {inlet }}$. The time scalar $\tau$ was $0.03 \mathrm{~s}$ for all cases. The dashed black line represents the target range (minima and maxima). The gray line represents the actual minima and maxima.
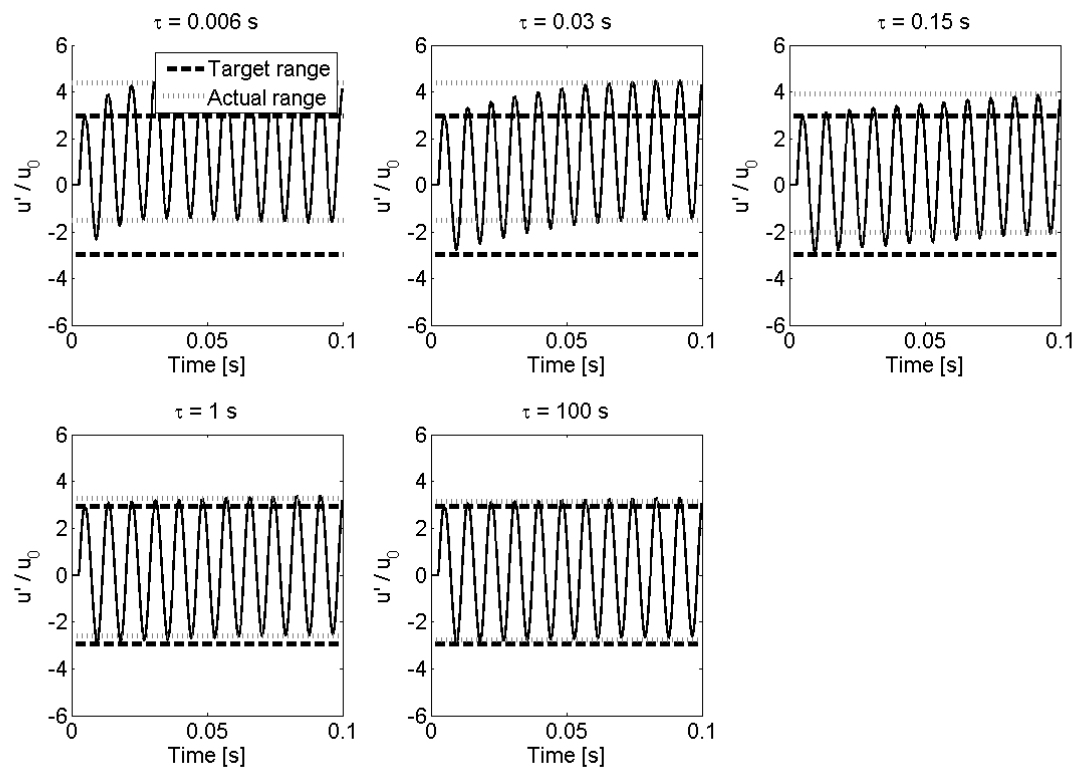

Figure 3.10: Drifting of the velocity, as function of the time scalar $\tau$. The frequency $f$ was $115 \mathrm{~Hz}$ for all cases and $\left|u^{\prime} / u_{0}\right|_{\text {inlet }}=2.95$. The dashed black line represents the target range (minima and maxima). The gray line represents the actual minima and maxima. 
- For simulations without excitation. The time scalar $\tau$ determines the cut-off frequency of the boundary condition and the time required for the mean values to converge to the target mean values. When large $\tau$-values are used, the simulation needs much longer to converge than for smaller values. After convergence, too large values of $\tau$ could also result in discrepancies between real and target values of the boundary condition: drifting.

- For simulations with large excitation amplitude. Since a converged mean flow solution has already been obtained, the time scalar $\tau$ does not imply a penalty on the required time for convergence. On the contrary, a large value of $\tau$ prevents the mean flow from converging to a different value. The fact that too large values of $\tau$ could also result in drifting is still true, but this drifting can only be observed after very long simulation times, that are much larger than the required length of the simulation.

These insights lead to the following boundary condition strategy: for simulations without excitation, the time scalar must be chosen as small as possible, but large enough to be in the non-reflecting range for the frequencies of interest $\left(f \cdot \tau=\mathcal{O}\left(10^{1}\right)\right.$ in figure 3.4). Only for simulations with large amplitude excitation the time scalar is then raised to a larger value, being an order of magnitude larger than the physical simulation time.

\subsection{Conclusions}

In this chapter a method to predict the Flame Describing Function from Computational Fluid Dynamics, more specifically Large Eddy Simulation, has been presented. The features of the unsteady reacting flow solver used in this work have been discussed in detail, as well as the specific requirements for thermo-acoustic applications. Specific requirements for the identification of the Flame Describing Function are for instance the setup of non-reflecting boundary conditions with high amplitude excitation, sampling of acoustic properties and postprocessing in the frequency domain.

The method to predict the Flame Describing Function has not been validated yet. This validation is the topic of the next chapter. However, it has been shown that the code performs well for non-reacting thermo-acoustic applications. It has been shown that the code features low dissipation and dispersion characteristics and that high amplitude excitation of the boundary conditions is possible, which is required in order to obtain the FDF. Finally, the transfer matrix of an area discontinuity is successfully predicted in Appendix D, which indicates that acoustic propagation and reflection is correctly described by the CFD code. 


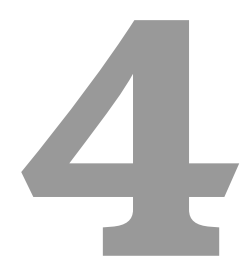

\section{Application to a generic atmospheric swirl flame}

\subsection{Introduction}

The goal of this chapter is to validate the combined numerical modeling approach for limit cycle prediction. This approach consists of first determining the Flame Describing Function from CFD and then to predict the limit cycle pressure amplitude based on this simulated Flame Describing Function. This validation is done in the context of a swirl-stabilized premixed flame.

The test rig is located at the Chair of Fluid Dynamics at the University of Berlin. All experimental data that will be used in this chapter were obtained by Schimek et al. $[85,86]$. In section 4.2 , a description of the test case and the experimental methods will be given. This is followed by a discussion of the CFD model in section 4.3.

As mentioned in Chapter 3, first a stable and converged reacting mean flow solution needs to be determined. In section 4.4, the mean flow solution and comparison against experimental data will be presented. After obtaining the mean flow solution, the flow is excited and the Flame Describing Function is determined in section 4.5. This is done for two operating conditions. From these Flame Describing Functions and CFD simulations, the mechanism leading to saturation of the heat release response is identified as well (section 4.6).

After obtaining the Flame Describing Function, the limit cycle pressure amplitude is predicted with the Generalized Instability Model in section 4.7. Both GIM simulations using the measured and simulated Flame Describing Functions have been performed. For both operating points the results are compared against measured pressure spectra.

As discussed in Chapter 1 (see figure 1.3), another method to study thermo- 
acoustic instabilities is the self-excited method, in which the CFD simulation becomes unstable itself and no separate forcing at the boundaries is required. This method has been investigated as well and will be discussed in section 4.8. The results obtained with this self-excited method and the results obtained with the Generalized Instability Model will finally be compared.

\subsection{Description of test case}

In figure 4.1 a schematic drawing of the rig is shown. The burner consists of a swirl generator followed by an annular duct and an area discontinuity to the combustion chamber, where a vortex breakdown is established. This leads to a recirculation zone and thus to a stabilization of the flame. In table 4.1 the main dimensions of the rig are summarized.

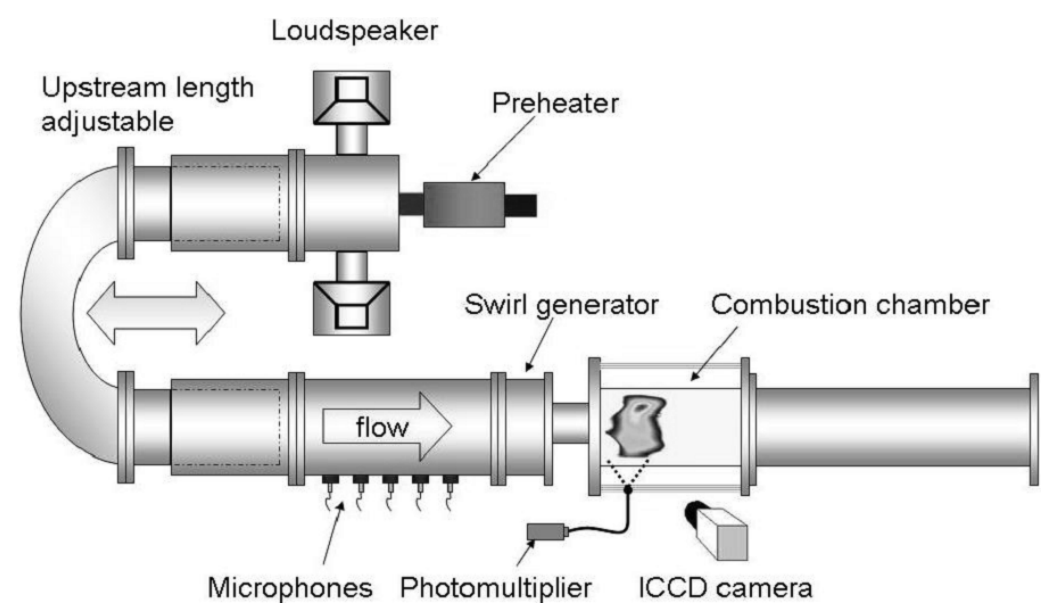

Figure 4.1: Schematic drawing of the test rig [86].

The air is preheated upstream of the swirl generator to $220^{\circ} \mathrm{C}$. The air flowing out of the preheater is choked (by using a nozzle), which prevents acoustic waves from traveling into the preheat system. Four speakers are mounted in the upstream duct, providing the acoustic forcing. The length of the upstream section could be continuously varied between 2.0 and 2.7 meters, which was used to create resonance in the upstream section. Resonance in the upstream section was required to achieve the required excitation amplitudes at specific frequencies. The first resonance frequency of the upstream section was in the order of $50 \mathrm{~Hz}$, the second around $100 \mathrm{~Hz}$. Between approximately 60 and $100 \mathrm{~Hz}$, no resonance could be created by changing the length of the upstream section. Above $100 \mathrm{~Hz}$, the resonance frequencies could be continuously varied [85]. By creating resonance in the upstream section, the setup is able to excite the acoustic velocity at the flame with amplitudes of up to $100 \%$ of the mean flow velocity (except for frequencies between 60 and $100 \mathrm{~Hz}$ ).

The setup was both run in partially premixed (or technically premixed) and perfectly premixed mode. For partially premixed combustion operation, fuel (natural 


\begin{tabular}{lc}
\hline Quantity & Dimension \\
\hline Outer diameter annular duct & $0.055 \mathrm{~m}$ \\
Inner diameter annular duct & $0.0275 \mathrm{~m}$ \\
Total length of annular duct & $0.171 \mathrm{~m}$ \\
Diameter combustion chamber & $0.105 \mathrm{~m}$ or $0.2 \mathrm{~m}$ \\
Total length of combustion chamber & $1.5 \mathrm{~m}$ \\
Diameter of upstream duct & $0.1484 \mathrm{~m}$ \\
Length of upstream duct & $2.0 \mathrm{~m}-2.7 \mathrm{~m}$ \\
\hline
\end{tabular}

Table 4.1: Main dimensions of rig.

gas) was injected through sixteen fuel nozzles located in the swirl generator. For the perfectly premixed operation, air and fuel were mixed far upstream of the swirler. A cross-section of the swirler with the location of the fuel injection is shown in figure 4.2 .

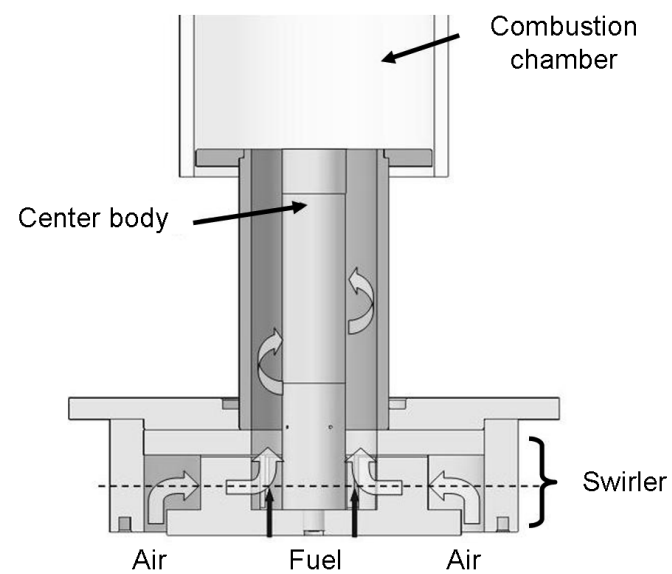

Figure 4.2: Cross-section of swirler [86]. In the partially premixed operating mode, fuel is axially injected through 16 nozzles located in the swirler. In the perfectly premixed operating mode, air and fuel are mixed upstream of the swirler.

The heat release rate of the flame was measured using $O H^{*}$ chemiluminescence. Upstream of the swirl generator, an array of microphones was mounted. The acoustic field was determined from these microphones using the multi-microphone method [85]. Schimek et al. both modeled and measured the transfer matrix of the swirl generator (see $[85,87])$, making it possible to calculate the velocity fluctuation at the combustion chamber inlet based on the acoustic field upstream of the swirl generator. Schimek found that this approach is both valid for small and large excitation amplitudes [87].

Schimek et al. defined their FDF according to equation 4.1:

$$
\mathcal{F}\left(\omega,\left|u^{\prime}\right|\right)=\frac{\hat{I}_{O H^{*}} / I_{O H^{*} 0}}{\hat{u} / u_{0}}
$$


Comparing equation 4.1 to the definition of the FDF in equation 1.5 (see Chapter 1), it can be seen that the volume integrated $O H^{*}$ intensity $I_{O H^{*}}$ is assumed to be proportional to heat release fluctuations $\left(\hat{Q} / Q_{0}=\hat{I}_{O H^{*}} / I_{O H^{*} 0}\right)$. For perfectly premixed combustion, it is known that this assumption introduces only slight errors (see for instance Schuermans et al. [88]). For the partially premixed operating mode on the other hand, where equivalence ratio oscillations occur, there may be a strong non-linearity between $Q$ and $I_{O H^{*}}$. This nonlinearity has been observed by several research groups, see e.g. [14,45,58,59].

The formation of $O H^{*}$ radicals is not predicted by the CFD code. Therefore the FDF that will be determined from CFD is defined as in equation 1.5 and is compared against experimental data obtained via equation 4.1. The main implications of this comparison will become clear in section 4.5 .

The Flame Describing Function was measured for different operating conditions (swirl numbers, air mass flows, preheat temperature, equivalence ratio) and for the partially and perfectly premixed operating modes. Two operating conditions were studied: a perfectly premixed and a partially premixed operating condition. In table 4.2 the parameters for the operating points are shown. The perfectly premixed case was studied with a combustion chamber with a larger diameter, because only for this larger diameter combustion chamber PIV measurement data was available.

\begin{tabular}{lcc}
\hline Operating condition & Perfectly premixed & Partially premixed \\
\hline Swirl number & 1.2 & 1.2 \\
Air mass flow & $0.0417 \mathrm{~kg} / \mathrm{s}$ & $0.0417 \mathrm{~kg} / \mathrm{s}$ \\
Air preheat temperature & $220^{\circ} \mathrm{C}$ & $220^{\circ} \mathrm{C}$ \\
Equivalence ratio & 0.65 & 0.65 \\
Diameter combustion chamber & $0.2 \mathrm{~m}$ & $0.105 \mathrm{~m}$ \\
\hline
\end{tabular}

Table 4.2: Investigated operating points.

During measurements of the Flame Describing Function, a orifice with a diameter of approximately $0.0375 \mathrm{~m}$ was mounted at the exit of the combustion chamber, which reduced the cross-sectional area by a factor 8 . This significantly changed the reflection coefficient of the outlet and suppressed self-excited instabilities. When this orifice was removed, the combustor became unstable for the partially premixed case. For the perfectly premixed operating condition the combustor was also stable without the orifice.

The perfectly and partially premixed operating conditions have been studied for different reasons:

- Perfectly premixed mode: The main objective is to validate the identification of the Flame Describing Function from CFD. As discussed, the perfectly premixed case does not suffer from the nonlinearity between $Q$ and $O H^{*}$ fluctuations, which makes this case more suited for a direct quantitative comparison. Also the mean flow field will be compared against PIV data and the saturation mechanism will be studied. 
- Partially premixed mode: As discussed above, self-excited instabilities occurred without the orifice at the exit. Pressure spectra have been measured during self-excited operation of the rig. The main objective of the partially premixed case is to validate the prediction of the limit cycle pressure amplitude using a combined application of the Flame Describing Function obtained from CFD and the nonlinear stability code (discussed in Chapter 2). For the prediction of the Flame Describing Function from CFD, only a qualitative comparison between the measured and simulated FDF's can be done, because of the nonlinearity between $O H^{*}$ and heat release rate $Q$ that has been discussed above.

\subsection{CFD Setup}

In figure 4.3 the computational grid of the perfectly premixed case is shown, as well as a more detailed view of the swirl generator. The grid was generated with OpenFOAM's mesh generator SnappyHexMesh. It consists of approximately 3.7 million hexahedral cells and contains the full $360^{\circ}$ burner geometry. It can be seen that regions with varying mesh density have been specified. This way the most important parts of the geometry are accurately resolved, while the less critical sections do not require as many cells. The swirler vane passages contain about 8 cells over the passage $(0.25 \mathrm{~mm}-0.5 \mathrm{~mm}$ cell size). The annular duct upstream of the flame contains about 15 cells over the radius (cell size about $1 \mathrm{~mm}$ ). The cell size in the flame region is about $2 \mathrm{~mm}$ and becomes courser further downstream.

The upstream section with the loudspeakers was not fully modeled, but the CFD Model starts $30 \mathrm{~cm}$ upstream of the swirl generator. It was assumed that the flow is one-dimensional at this location.

The computational grid and domain for the partially premixed case are similar. The main differences are the smaller diameter of the combustion chamber and the additional fuel nozzle inlet boundary conditions (see figure 4.2 for the location of these nozzles).

In the simulation, non-reflecting boundary conditions were used for the velocity, pressure, and temperature at the inlet and outlet, which assured that acoustic waves leave the domain. The walls of the combustor were assumed to have a constant temperature of $800 \mathrm{~K}$. This is indicated in figure 4.3 as well. The exact temperature of the wall was not known, but this estimation will be closer to the actual temperature than it would be if adiabatic walls were used. Tay et al. [12] showed that the modeling of heat losses through the walls is very important for accurate predictions of the heat release distribution, and thus of the predicted flame transfer functions. In his simulations, the inclusion of these heat losses resulted in a longer flame and a larger phase between velocity and heat release fluctuations. The influence of the wall temperature on the FDF of the swirl stabilized burner presented in this chapter was small however.

Both the spatial and temporal derivatives were discretizised using $2^{\text {nd }}$ order discretization schemes (see section 3.2.6). A fixed time step of $d t=5 \cdot 10^{-7} \mathrm{~s}$ was chosen, assuring that the convective Courant number was below $C o=0.3$ everywhere in the domain and the acoustic CFL number was below $C F L=1$. This guaranteed both 

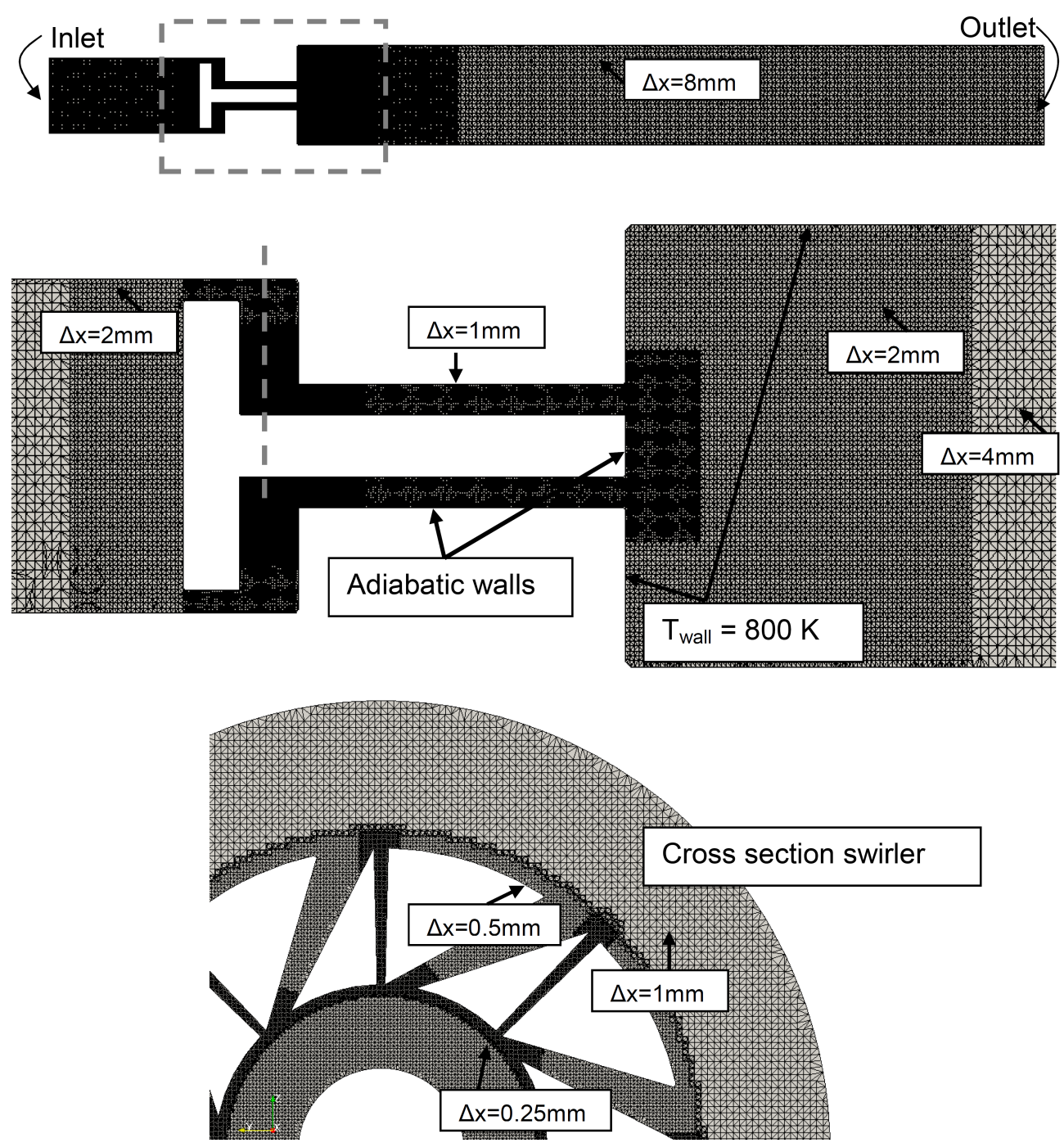

Figure 4.3: Grid and boundary conditions for the perfectly premixed case. The cell size is indicated as well.

robustness of the simulation and negligible dissipation of acoustic waves.

In the present work, first a LES without excitation was performed. After obtaining a converged reacting LES, the Flame Describing Function was determined, by exciting the velocity at the air inlet using mono-frequency harmonic waves. For the partially premixed case, only the air inlet was excited, the fuel mass flow was kept constant (as was also done in the experiments). Finally, a self-excited simulation was done as well.

The parameters of the non-reflecting boundary conditions (see equation 3.40) can 
be found in table 4.3 .

\begin{tabular}{lcc}
\hline Type of simulation & Time scalar inlet & Time scalar outlet \\
\hline Mean flow field (Sec. 4.4) & $\tau=0.03 \mathrm{~s}$ & $\tau=0.03 \mathrm{~s}$ \\
Flame Describing Function (Sec. 4.5) & $\tau=1 \mathrm{~s}$ & $\tau=0.03 \mathrm{~s}$ \\
Self-excited simulation (Sec. 4.8) & $\tau=3 \cdot 10^{-5} \mathrm{~s}$ & $\tau=3 \cdot 10^{-5} \mathrm{~s}$ \\
\hline
\end{tabular}

Table 4.3: Non-reflection boundary condition time scalars used in the different kinds of simulations. The sections in which these simulations are discussed is indicated between parentheses.

The time scalar was chosen such that for the mean flow field simulation, all frequencies larger than $60 \mathrm{~Hz}$ are reflected less than 10\% (see figure 3.4). For Flame Describing Function simulations, the time scalar at the inlet was raised in order to prevent drifting of the mean velocity (see section 3.4.2) and for self-excited simulations, the time scalar was set to a value of $\tau=3 \cdot 10^{-5} \mathrm{~s}$. This resulted in a reflection coefficient of $R_{\text {outlet }}=-0.99$ in the self-excited simulation, corresponding to the measured reflection coefficient (see also section 4.8).

\subsection{Steady flow field results}

In figure 4.4 the results of the simulation without excitation of the boundary conditions can be seen, for the perfectly premixed case. In figure 4.4(a) the time-averaged axial velocity field is shown. The white line represents a contour of $u_{a x}=0 \mathrm{~m} / \mathrm{s}$, indicating the boundaries of the recirculation regions. Both an outer recirculation zone in the corner of the combustion chamber and a strong inner recirculation zone were observed in the simulations. A contour of the reaction progress variable $(\tilde{c}=0.9)$ has been added to the figure as well to indicate the location of the flame front. The flame stabilizes in the region between the inner and outer recirculation zone.

The instantaneous temperature field is shown in figure 4.4(b). Again a contour of the reaction progress variable has been added to indicate the location of the flame front. Compared to the contour of the time averaged progress variable, it can be seen that the flame is highly wrinkled.

The time-averaged velocity field is compared against Particle Image Velocimetry (PIV) measurements in figure 4.5. The flow field is compared at different axial locations, where the coordinate $x$ represents the distance from the inlet of the combustion chamber (as indicated by the markers in figure 4.4). The mean axial velocity component is compared in figure 4.5(a). The simulation generally shows good agreement with the measurements. The location and magnitude of the peak velocities are well predicted, as well as the central recirculation zone. The velocity close to the walls of the combustor is over predicted in the simulation, especially for $x<0.05 \mathrm{~m}$. According to private communication with Schimek et al., there may be some inaccuracies in the measurement close to the walls due to contamination of the windows [55]. Another reason for this may be the wall law used by the LES turbulence model.

The radial component of the velocity field is compared against PIV data in figure 4.5(a). Also for the radial velocity, the peak values and central recirculation zone 


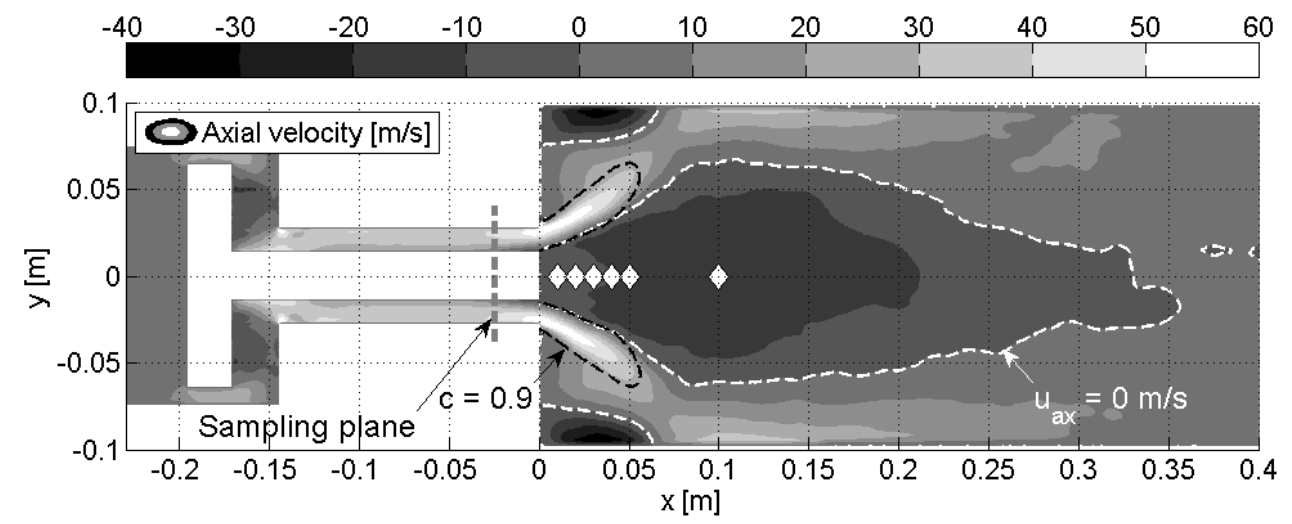

(a) Contour plot of the axial velocity, averaged over $40 \mathrm{~ms}$. White line represents contour of $u_{a x}=0 \mathrm{~m} / \mathrm{s}$. Black line represents an iso-contour of the reaction progress variable $(\tilde{c}=0.9)$. Grey line represents the plane where velocity fluctuations for the FDF have been sampled. White markers indicate axial locations where comparison with PIV data has been made (see figure 4.5).

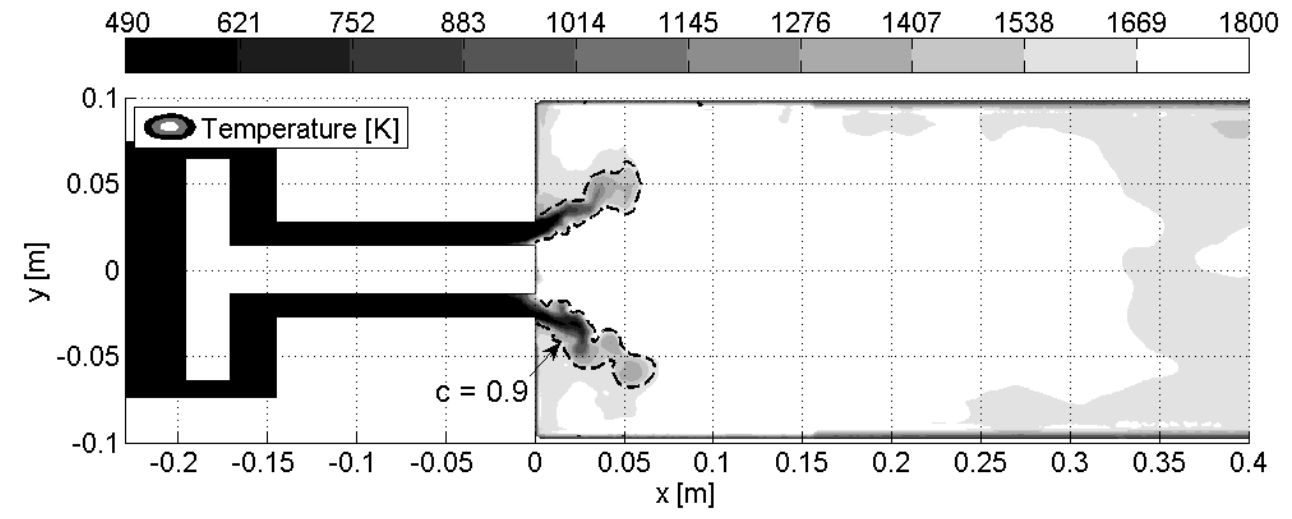

(b) Instantaneous temperature field inside the combustion chamber. Black line represents an iso-contour of the reaction progress variable $(\tilde{c}=0.9)$.

Figure 4.4: Steady flow field results for the perfectly premixed case. 

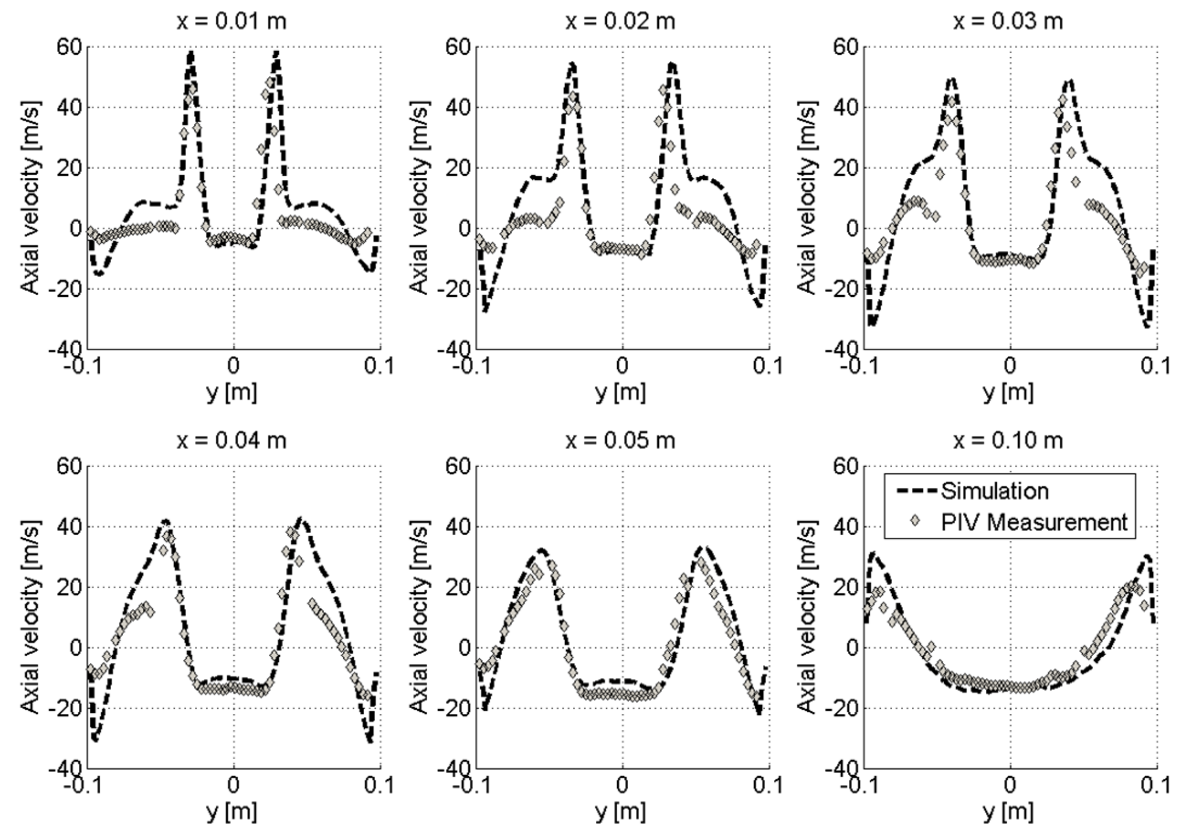

(a) Axial velocity.
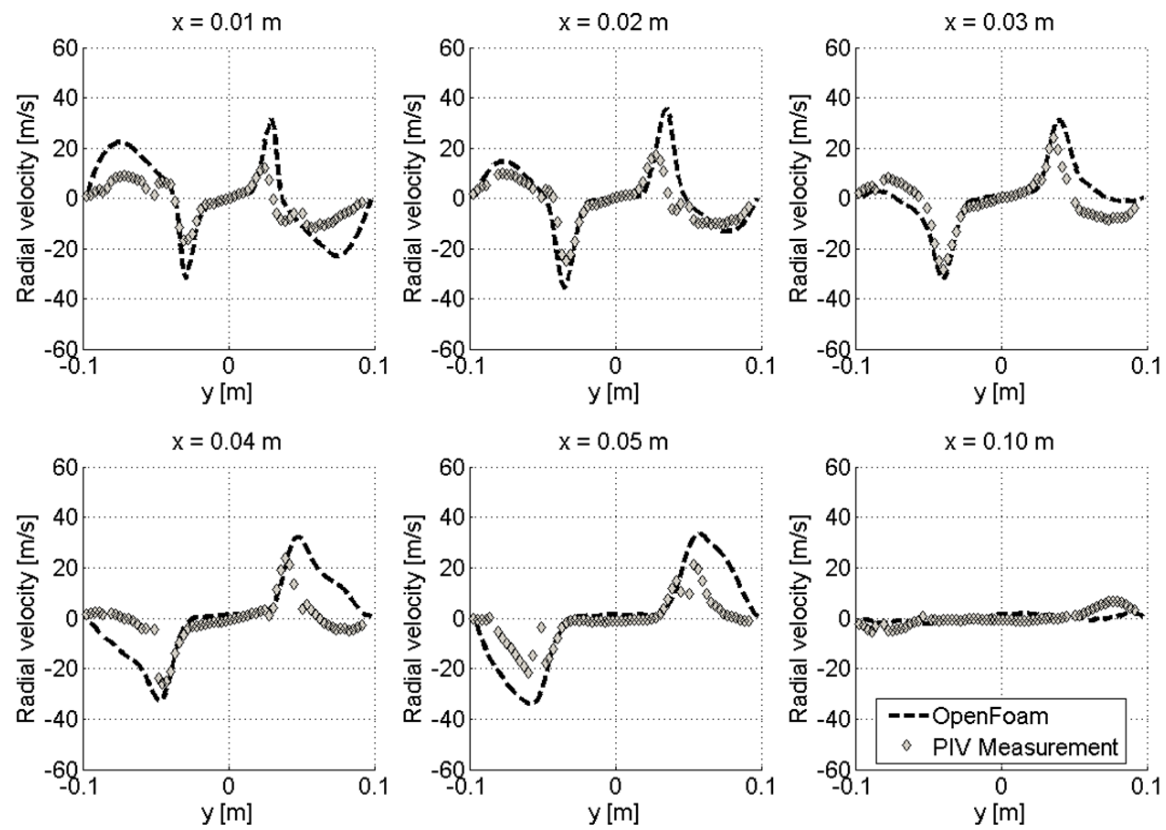

(b) Radial velocity.

Figure 4.5: Comparison of mean predicted velocity against PIV Measurement at different axial locations from the combustion chamber inlet ( $x$ is indicated by the white markers in figure 4.4(a)), for the perfectly premixed case. 
are well predicted, but there is some over prediction in the outer recirculation zone (especially for $x=0.01 \mathrm{~m}, x=0.04 \mathrm{~m}$ and $x=0.05 \mathrm{~m}$ ).

The perfectly premixed case was also numerically studied by CERFACS. In Hermeth et al. [39] the flow fields obtained from their LES code AVBP are compared against the results presented in this section and PIV measurements. In Appendix F a comparison between the CFD setups and mean flow fields is provided.

The flow fields for which no experimental data was available (temperature, tangential velocity and root mean square fields) are also provided in Appendix F.

\subsection{Flame Describing Function from CFD}

After obtaining the mean flow solution, the inlet boundary condition has been excited with mono-frequency harmonic waves (see equation 3.40). Both the frequency and amplitude of the harmonic wave have been varied in order to obtain the Flame Describing Function. The sampling plane for the acoustic velocity fluctuations is located slightly upstream of the entrance to the combustion chamber, the location of the sampling plane is indicated in figure 4.4(a).

In order to obtain a certain level of excitation at this reference plane, a much larger excitation level at the inlet boundary had to be applied. In figure 4.6(a) an example of a typical time series upstream and downstream of the swirler can be seen. It can be seen that in order to reach $u^{\prime} / u_{0} \approx 0.1$ in the annular duct between the swirler and the flame, the velocity upstream of the swirler had to be perturbed by $u^{\prime} / u_{0_{\text {upstream }}} \approx 0.4$. This means that about $75 \%$ of the acoustic wave that impinges on the swirler is dissipated inside the swirler or reflected back towards the inlet. Only $25 \%$ of the acoustic wave is transmitted. This has also been observed by Schimek et al. and is in agreement with transfer function measurements of the swirler [87].

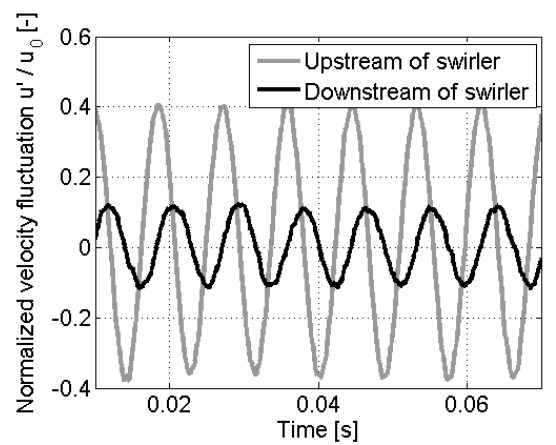

(a) Velocity fluctuations upstream and downstream of the swirler.

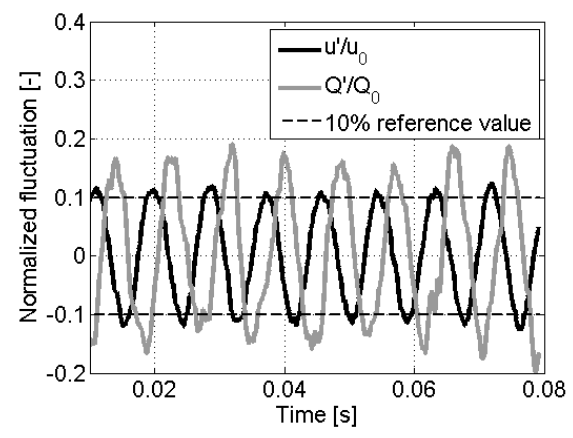

(b) Velocity fluctuations (downstream of the swirler) and heat release fluctuations.

Figure 4.6: Example of normalized fluctuations at a frequency of $115 \mathrm{~Hz}, u^{\prime} / u_{0} \approx 0.1$ for the perfectly premixed case.

In figure 4.6(b) an example of a typical time series of the velocity perturbation upstream of the flame and the volume integrated heat release fluctuations is presented. 
This example shows the response at a frequency of $115 \mathrm{~Hz}$ and an acoustic velocity with an amplitude equivalent to $10 \%$ of the mean flow velocity. Both quantities have been normalized by their mean values. Clearly a phase difference and a difference in amplitude can be seen between the normalized velocity and heat release fluctuations.

As explained in section 3.3.3, for each harmonic simulation the amplitude and phase of the Flame Describing Function are determined by first calculating the Discrete Fourier Transform of the velocity and heat release fluctuations and then applying equation 3.52 .

\subsubsection{Perfectly premixed case}

In figure 4.7 the measured and simulated FDF for the perfectly premixed case are compared. Measurement data was available for 7 different excitation amplitudes $\left(u^{\prime} / u_{0}=0.1,0.2,0.3,0.4,0.5,0.6,0.7\right)$ but only the data for $u^{\prime} / u_{0}$ equal to $0.1,0.4$ and 0.7 has been modeled. For the excitation level $u^{\prime} / u_{0}=0.1$, six distinct frequencies have been simulated. For the larger excitation amplitudes, only simulations at 115 and $258 \mathrm{~Hz}$ have been performed. The frequencies and amplitudes for which simulations have been performed are shown in table 4.4. The inlet boundary excitation is given as well.

\begin{tabular}{ccc}
\hline $\begin{array}{c}\text { Excitation amplitude } \\
\text { at flame }\end{array}$ & $\begin{array}{c}\text { Excitation amplitude } \\
\text { at inlet boundary }\end{array}$ & Frequencies \\
\hline$u^{\prime} / u_{0}=0.1$ & $u^{\prime} / u_{0}=0.4$ & $95 \mathrm{~Hz}, 115 \mathrm{~Hz}, 144 \mathrm{~Hz}$, \\
& & $182 \mathrm{~Hz}, 258 \mathrm{~Hz}, 323 \mathrm{~Hz}$ \\
\hline$u^{\prime} / u_{0}=0.4$ & $u^{\prime} / u_{0}=1.6$ & $115 \mathrm{~Hz}, 258 \mathrm{~Hz}$ \\
\hline$u^{\prime} / u_{0}=0.7$ & $u^{\prime} / u_{0}=2.8$ & $115 \mathrm{~Hz}, 258 \mathrm{~Hz}$ \\
\hline
\end{tabular}

Table 4.4: Harmonic frequency simulations for the perfectly premixed case.

The simulations generally agree well with the measurements. The amplitude of the FDF is predicted well, especially for frequencies larger than $150 \mathrm{~Hz}$. The maximum amplitude of the FDF is predicted at $115 \mathrm{~Hz}$ but at a slightly lower value than the measurement. The measured amplitude was about $|\mathcal{F}|=2.0$ versus $|\mathcal{F}|=1.6$ in the simulation (the difference between simulation and measurement is also shown in figure 4.9(a)).

At the frequency of $115 \mathrm{~Hz}$, simulations at 3 different excitation amplitudes have been performed. It can be seen that the amplitude of the FDF reduces for the larger excitation amplitudes. This means that the response of the flame weakens for larger velocity amplitudes. This can be related to the energy balance sketched in figure 1.4 by considering that the FDF corresponds to the slope of the gain curve. From this it can be concluded that the nonlinear saturation of the flame is captured by the simulation. At a frequency of $258 \mathrm{~Hz}$, however, the flame did not show any saturation in simulation and experiment for the excitation amplitudes considered. The mechanism leading to the saturation of the FDF is investigated in section 4.6. 


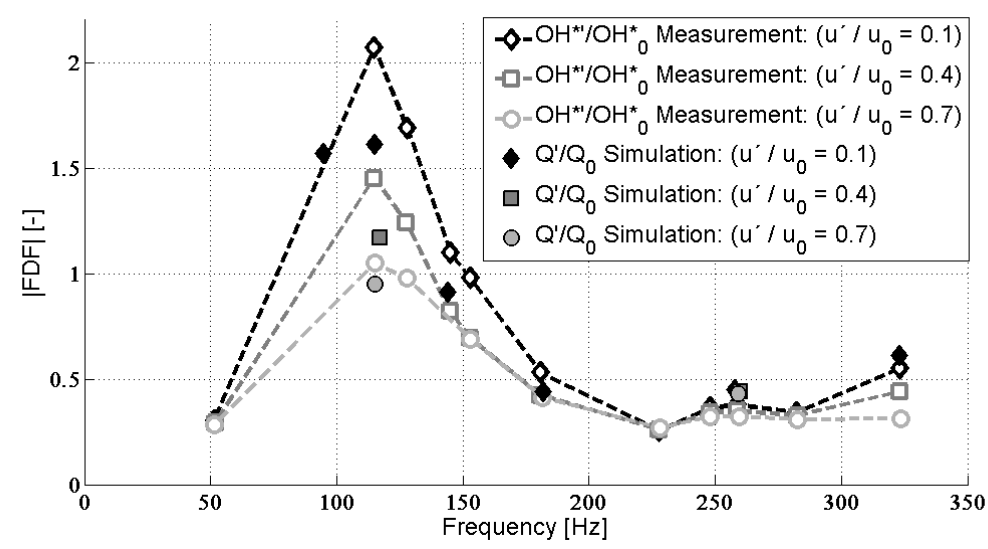

(a) Magnitude.

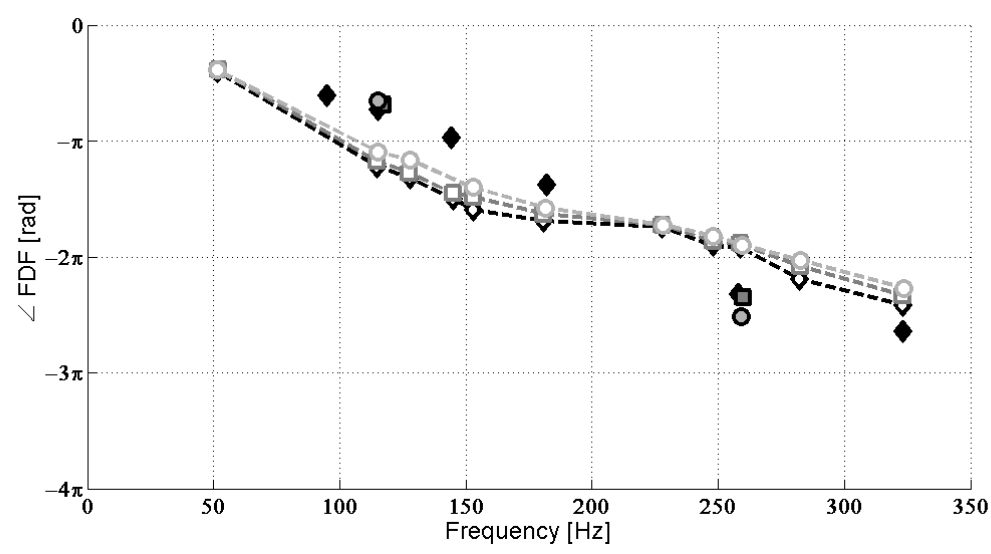

(b) Phase.

Figure 4.7: Comparison of measured and simulated Flame Describing Function for the perfectly premixed case. 


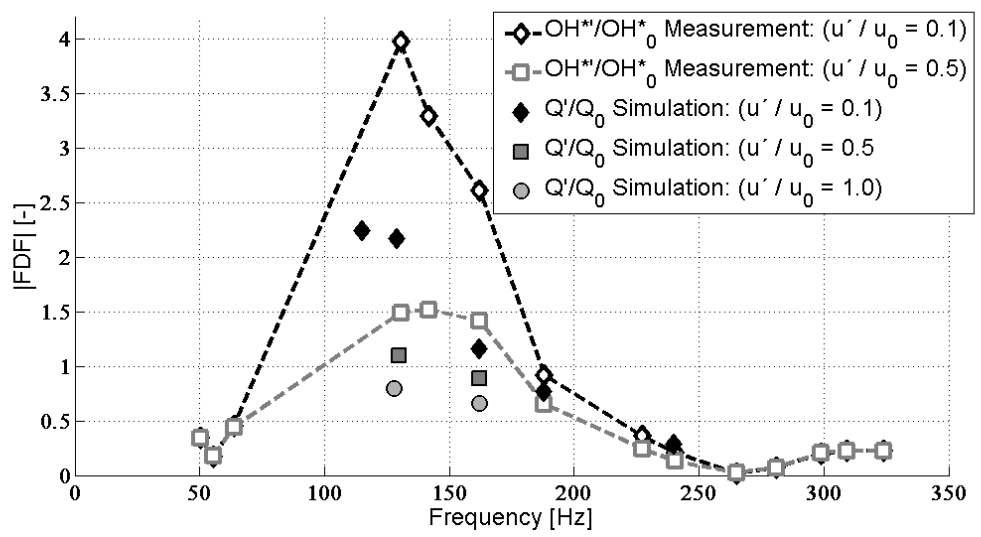

(a) Magnitude.

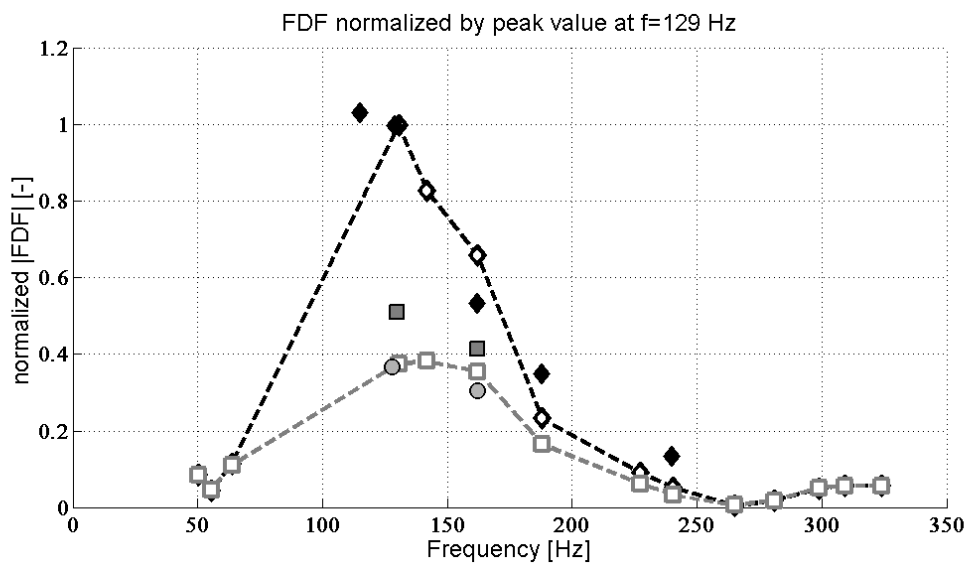

(b) Magnitude normalized by peak value of $129 \mathrm{~Hz}$ in figure 4.8(a). The goal of this graph is a qualitative comparison between numerical and experimental data.

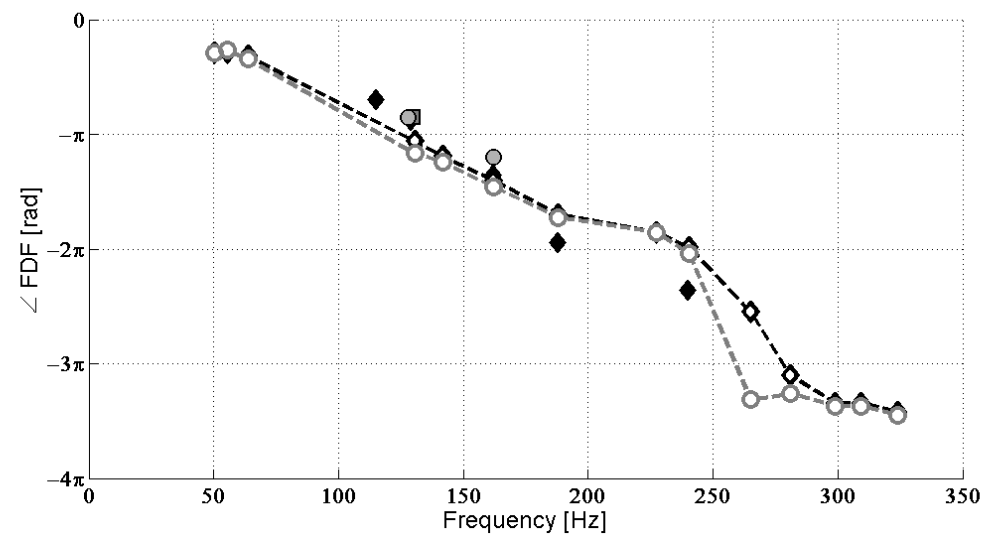

(c) Phase.

Figure 4.8: Comparison of measured and simulated Flame Describing Function for the partially premixed case. 


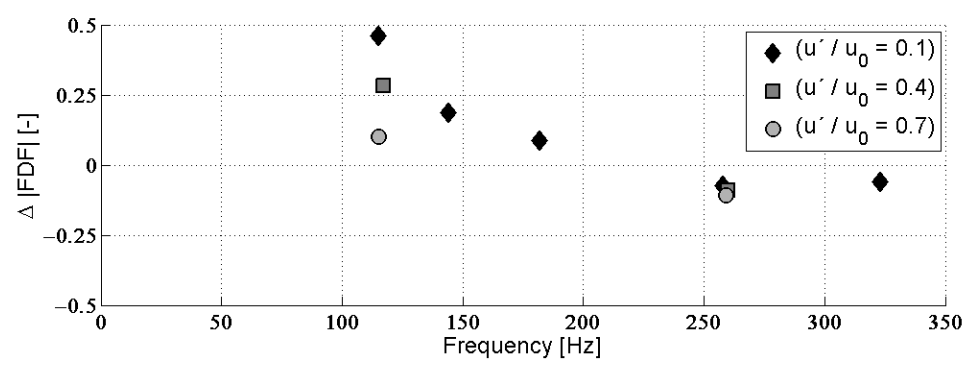

(a) Magnitude $\left(|\mathcal{F}|_{\text {meas }}-|\mathcal{F}|_{C F D}\right)$.

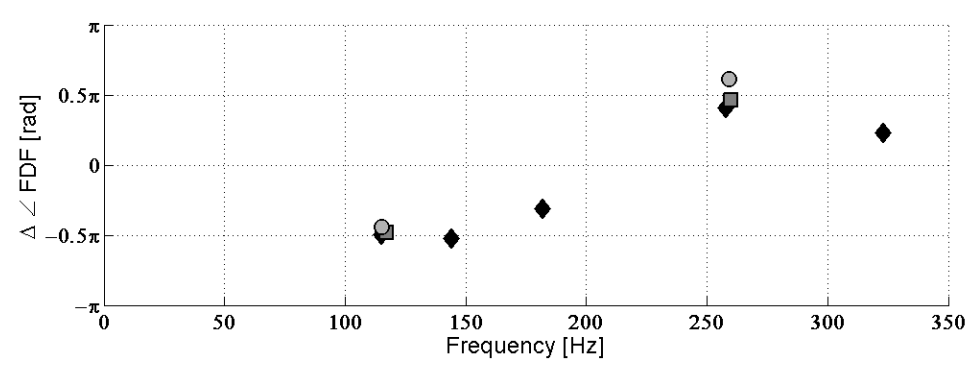

(b) Phase $\left(\angle \mathcal{F}_{\text {meas }}-\angle \mathcal{F}_{C F D}\right)$.

Figure 4.9: Difference plot: Perfectly premixed case.

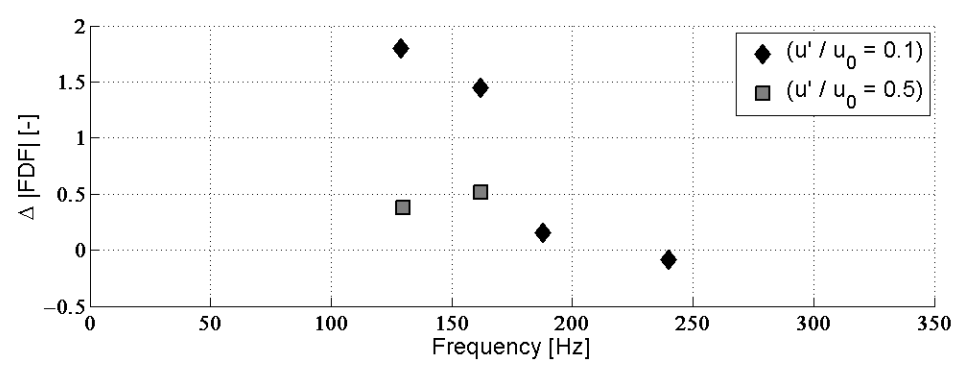

(a) Magnitude $\left(|\mathcal{F}|_{\text {meas }}-|\mathcal{F}|_{C F D}\right)$.

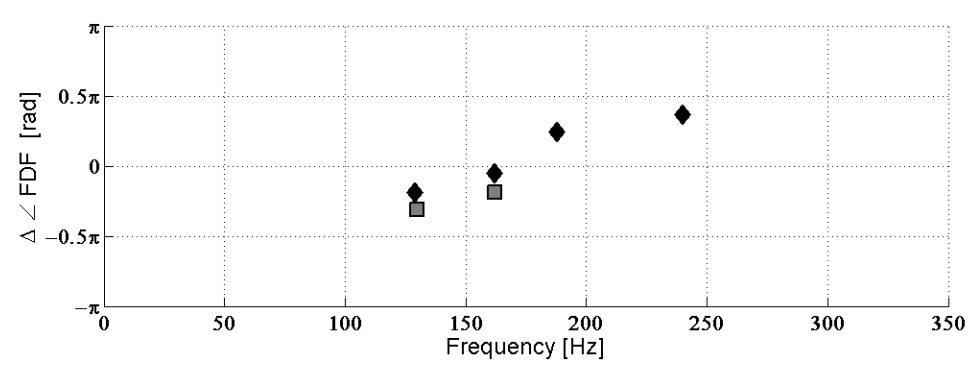

(b) Phase $\left(\angle \mathcal{F}_{\text {meas }}-\angle \mathcal{F}_{C F D}\right)$.

Figure 4.10: Difference plot: Partially premixed case. 
In the measurements, also a minimum gain was found at approximately $50 \mathrm{~Hz}$. The frequency at $50 \mathrm{~Hz}$ has not been simulated. The reason for this is that this frequency is about a factor 3 smaller than the lowest eigenfrequency of the combustion chamber and thus of little relevance. Furthermore the required simulation time increases inversely proportional to the frequency (at least 8 periods of oscillation have to be simulated in order to calculate a reliable Discrete Fourier Transform), which would make this a very expensive calculation.

When looking at the phase plot, for frequencies below $200 \mathrm{~Hz}$ the phase is under predicted, by approximately $0.4 \pi$ at $\mathrm{f}=115 \mathrm{~Hz}$ and $0.2 \pi$ at $182 \mathrm{~Hz}$ (see figure $4.9(\mathrm{~b})$ ), whereas the phase is slightly over predicted for the larger frequencies. Nonetheless, the general agreement between measurements and simulation is good. The phase decreases approximately linearly with frequency, which indicates that the flame has a constant time lag (in this case $\tau \approx 3.95 \mathrm{~ms}$ ). The phase did not appear to be dependent on the excitation amplitude, both in simulation and experiments.

From the comparison between simulations and experiments, it can be concluded that the magnitude of the Flame Describing Function and the saturation of the heat release for large perturbation amplitudes can successfully be predicted using Large Eddy Simulations. In order to quantify the effect of the differences between measurements and simulations in the phase of the FDF, a stability analysis is required. This stability analysis is the topic of section 4.7 .

\subsubsection{Partially premixed case}

In figure 4.8 the measured and simulated FDF for the partially premixed case are compared. The FDF was determined in exactly the same way as the FDF of the perfectly premixed case and thus is a Single Input, Single Output (SISO) FDF. As discussed in section 1.2.4 of the introduction chapter, partially premixed systems can also be characterized using MISO models. This was not done however, because the measured FDF is also a SISO FDF. The MISO formulation will be tested in Chapter 5 .

For the partially premixed case, measurement data was available for $\left(u^{\prime} / u_{0}=\right.$ $0.1,0.2,0.3,0.4,0.5)$. Simulations have been done for $u^{\prime} / u_{0}$ equal to $0.1,0.5$ and 1.0. The large excitation amplitude of $u^{\prime} / u_{0}=1.0$, for which no measurement data was available, was required for the limit cycle prediction using the nonlinear stability code. For the excitation level $u^{\prime} / u_{0}=0.1$, five distinct frequencies have been simulated. For the larger excitation amplitudes, only simulations at 129 and $162 \mathrm{~Hz}$ have been performed. The frequencies and amplitudes for which simulations have been done are shown in table 4.5. The simulations were done at slightly different frequencies than for the perfectly premixed case, because the measurements were not performed at exactly the same frequencies as well.

In figure 4.8(a) the magnitude of the FDF is compared. The general shape of the curve looks similar to the magnitude of the perfectly premixed case (figure 4.7(a)). Both the perfectly and partially premixed case have a peak value at approximately the same frequency $(115$ and $129 \mathrm{~Hz})$. For larger frequencies, the magnitude of the FDF reduces to small values. However, the peak magnitude of the partially premixed case is larger than the magnitude of the perfectly premixed case, both in experiments and simulations. 


\begin{tabular}{ccc}
\hline $\begin{array}{c}\text { Excitation amplitude } \\
\text { at flame }\end{array}$ & $\begin{array}{c}\text { Excitation amplitude } \\
\text { at inlet boundary }\end{array}$ & Frequencies \\
\hline$u^{\prime} / u_{0}=0.1$ & $u^{\prime} / u_{0}=0.4$ & $115 \mathrm{~Hz}, 129 \mathrm{~Hz}, 162 \mathrm{~Hz}$, \\
& $188 \mathrm{~Hz}, 240 \mathrm{~Hz}$ \\
\hline$u^{\prime} / u_{0}=0.5$ & $u^{\prime} / u_{0}=2.0$ & $129 \mathrm{~Hz}, 162 \mathrm{~Hz}$ \\
\hline$u^{\prime} / u_{0}=1.0$ & $u^{\prime} / u_{0}=4.0$ & $129 \mathrm{~Hz}, 162 \mathrm{~Hz}$ \\
\hline
\end{tabular}

Table 4.5: Harmonic frequency simulations for the partially premixed case.

It can be seen that the magnitude of the FDF obtained via numerical simulations is significantly lower than the magnitude of the experimental data (about a factor 2) at $129 \mathrm{~Hz}$. This is not an unexpected result. As discussed in section 4.2, the numerical FDF was calculated using equation 3.52, whereas the experimental FDF was calculated with equation 4.1. For the partially premixed operating mode, where equivalence ratio oscillations occur, there may be a strong nonlinearity between the heat release rate and $O H^{*}$ intensity.

In order to better compare the numerical and experimental data in a qualitative way, the FDF has been normalized by its peak value at $129 \mathrm{~Hz}$ and $u^{\prime} / u_{0}=0.1$ in figure $4.8(\mathrm{~b})$. After the normalization procedure, the qualitative comparison agrees well. For the lowest excitation amplitude $\left(u^{\prime} / u_{0}=0.1\right)$, all numerical and experimental data between 129 and $240 \mathrm{~Hz}$ lie more or less on the same curve, which indicates the cut-off frequency of the FDF is correctly captured. For larger excitation amplitudes $\left(u^{\prime} / u_{0}=0.5\right.$ and $\left.u^{\prime} / u_{0}=1\right)$, the magnitude of the FDF reduces, indicating that the heat release response of the partially premixed case saturates as well.

Finally in figure 4.8(c) the phase is compared. Again the phase did not significantly change with the excitation amplitude. For frequencies of up to $150 \mathrm{~Hz}$, the phase is slightly smaller than the phase of the perfectly premixed case (figure 4.7(b)), whereas for frequencies above $150 \mathrm{~Hz}$, a larger phase was found. The difference between the phase of the partially and perfectly premixed case could be related to the contribution of equivalence ratio fluctuations, which are not present in the perfectly premixed case. This was not further investigated within this work however.

From the comparison between numerical and experimental data, it can thus be concluded that also for the partially premixed case, the FDF can be predicted using LES. However, similar to the perfectly premixed case, the effect of the differences between measurements and simulations needs to be quantified. The stability analysis using measured and simulated Flame Describing Function will be presented in section 4.7 .

\subsection{Investigation of the saturation mechanism}

Next it is investigated why the heat release response becomes nonlinearly dependent on the amplitude of velocity perturbations. This effect is referred to as saturation. As discussed in Chapter 1, several experimental investigations suggest that the heat 
release saturates because the flame surface area increases nonlinearly with excitation amplitude (a list of key references for this statement can be found in section 1.2.3). To test whether the LES results agree with these observations, the amplitude of the surface area fluctuations was extracted and compared to the amplitude of the heat release fluctuations and velocity fluctuations.

In Large Eddy Simulations, the flame wrinkling is partly resolved and partly modeled. The resolved flame surface $\left(A_{\text {res }}\right)$, the modeled sub grid flame surface $\left(A_{S G S}\right)$ and the total flame surface $\left(A_{t o t}\right)$ can be calculated by integrating the local Flame Surface Density $|\nabla \widetilde{c}|$ over the volume. They can be derived from the combustion model, see equation 3.24 , as follows:

$$
\begin{aligned}
A_{\text {res }}(t) & =\int|\nabla \widetilde{c}| d V \\
A_{\text {tot }}(t) & =\int \Sigma|\nabla \widetilde{c}| d V \\
A_{S G S}(t) & =\int(\Sigma-1)|\nabla \widetilde{c}| d V \\
Q(t) & =\int H_{f} \widetilde{\tilde{w}_{R}} d V=\int H_{f} \overline{\rho_{u}} S_{L}^{0} \Xi \Sigma|\nabla \widetilde{c}| d V
\end{aligned}
$$

The resolved and total flame surfaces are schematically shown in figure 4.11.

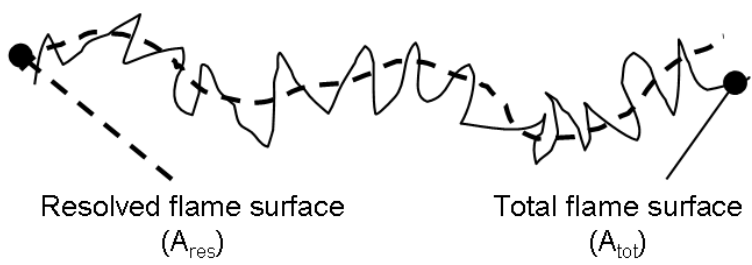

Figure 4.11: Sketch of the flame front. Thick dashed line shows the resolved flame surface $A_{\text {res }}$, thin line the total flame surface $A_{\text {tot }}$.

In equation $4.5, H_{f}$ is the enthalpy of formation. The dominant parameter causing the nonlinearity can be investigated by analyzing the different flame surface components (equation 4.2 to 4.4 ).

The amplitude of the fluctuations has been determined by taking the Discrete Fourier Transform of the time series obtained with equation 4.2 through 4.5. The investigation of these components is done for the perfectly premixed case and at a frequency of $115 \mathrm{~Hz}$, where the nonlinearity was found.

In figure 4.12 the resolved, modeled and total flame surface fluctuations are compared. The left plot (fig. 4.12(a)) shows the absolute value of the fluctuations. The total flame surface is the sum of the resolved and the modeled flame surface. The average wrinkling factor $\Sigma$ over the flame was in the order of 4-5, meaning that the total flame surface was about 4 to 5 times larger than the resolved flame surface. Referring to equation 3.26, the magnitude of $\Sigma$ is related to the cell size. Due to the close match of the calculated and experimentally found Flame Describing Function, the average value of $\Sigma$ is considered to be low enough. 


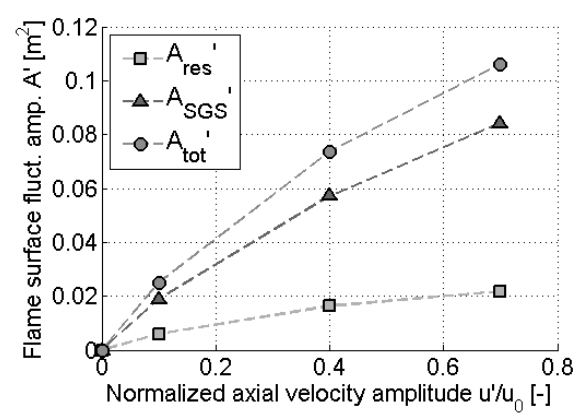

(a) Absolute

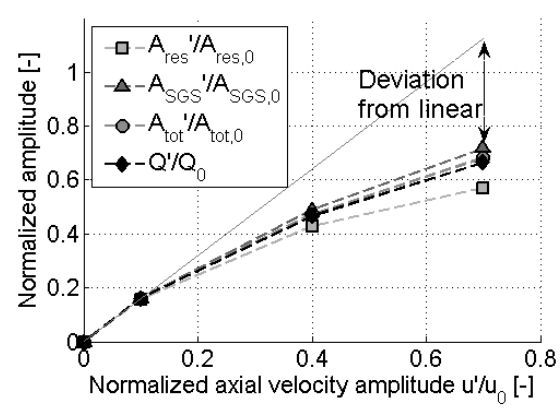

(b) Normalized

Figure 4.12: Amplitude of resolved $\left(A_{r e s}\right)$, subgrid $\left(A_{S G S}\right)$ and total $\left(A_{t o t}\right)$ flame surface fluctuations as function of the excitation amplitude for a frequency of $115 \mathrm{~Hz}$. Left plot shows absolute values, right plot normalized values. The thin line in the right figure shows the hypothetical linear curve that would be obtained if the heat release would not saturate.

In the right part of the figure (fig. 4.12(b)) the flame surface fluctuations have been normalized by their respective mean values. The heat release fluctuations are shown here as well. The deviation from a hypothetical linear flame response is obtained by extrapolating the heat release response for $u^{\prime} / u_{0}=0.1$ to larger excitation amplitudes. From this extrapolation the deviation from linear behavior can then be determined. All components showed a nonlinear dependency on the axial velocity amplitude.

The curves for the normalized total flame surface $\left(A_{t o t}^{\prime} / A_{t o t, 0}\right)$ and heat release $\left(Q^{\prime} / Q_{0}\right)$ are almost identical. From equation 4.3 and 4.5 it can be concluded that the saturation is not caused by nonlinearities in unburnt density $\left(\overline{\rho_{u}}\right)$, or strain effects $(\Xi)$. The unstrained laminar flame speed $\left(S_{L}^{0}\right)$ and $H_{f}$ are constant for perfectly premixed flames and do not have an effect here.

Next the differences between the resolved $\left(A_{\text {res }}^{\prime} / A_{\text {res }, 0}\right)$ and total flame surface $\left(A_{t o t}^{\prime} / A_{t o t, 0}\right)$ are investigated. For smaller excitation amplitudes both quantities have identical normalized amplitudes. For larger excitation amplitudes the normalized amplitude of the resolved part $\left(A_{\text {res }}^{\prime}\right)$ is slightly smaller. Looking at equation 4.2 and equation 4.3, however, and considering the deviation of the curves from linear behavior (also indicated in figure 4.12(b)), it can be concluded that the major part of the saturation of the heat release can be attributed to a nonlinear evolution of the term $|\nabla \widetilde{c}|$, which is the resolved Flame Surface Density (dimension $m^{-1}$ ), and only a small part to changes in the wrinkling factor $(\Sigma)$. This proves that FSD models are capable to capture the saturation mechanism.

As discussed in the introduction, previous experimental investigations suggest that for swirl flames, the heat release response is modulated by the interaction of axial and tangential fluctuations [71]. Depending on the phase difference between those fluctuations, the heat release response is either enhanced or damped. Using LES, the saturation of the heat release will be correlated to fluctuations of the individual velocity components.

In figure 4.13 the axial, tangential and swirl number fluctuations upstream of 


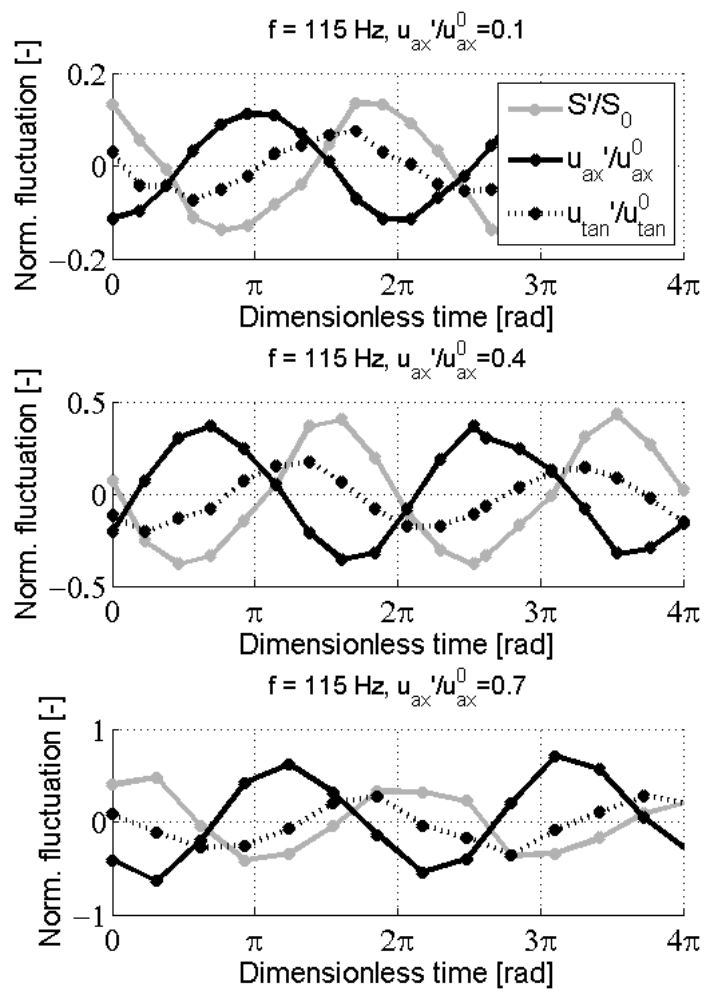

Figure 4.13: Axial, tangential and swirl number fluctuations at the inlet of the combustion chamber. All fluctuations are normalized by their mean values (indicated with subscript 0 )

the flame are shown for the different excitation amplitudes. The swirl number $S$ was defined as in equation 4.6 (Gupta et al. [36]). Swirl number fluctuations lead to fluctuations of the flame angle.

$$
S(t)=\frac{\int \bar{\rho} \tilde{u}_{t a n}(\mathbf{x}, t) \tilde{u}_{x}(\mathbf{x}, t) r(\mathbf{x}) d A}{R \int \bar{\rho} \tilde{u}_{x}^{2}(\mathbf{x}, t) d A}
$$

The area averaged tangential velocity fluctuation $u_{t a n}^{\prime}$ ans the swirl number fluctuation $S^{\prime}$ were defined analogous to the definition of the area averaged axial fluctuations (see section 3.3.2).* The time has been normalized with the frequency of oscillation, so that the phase difference between the fluctuations can easily be recognized. As can be seen, there is a significant difference in magnitude and phase between axial and tangential velocity fluctuations, which also explains the fluctuations in swirl number.

It is interesting to note that in figure 4.13 , when the excitation amplitude is increased, the amplitude of the swirl number fluctuations and tangential fluctuations

${ }^{*}$ The CFD code solves the transport equations in a Cartesian coordinate system. Since for this configuration $x$ is the direction of flow and the central axis of the combustor is given by $(y=0, z=0)$, the tangential velocity can be calculated as $u_{\tan }(\mathbf{x}, t)=\sqrt{u_{y}(\mathbf{x}, t)^{2}+u_{z}(\mathbf{x}, t)^{2}}$. 


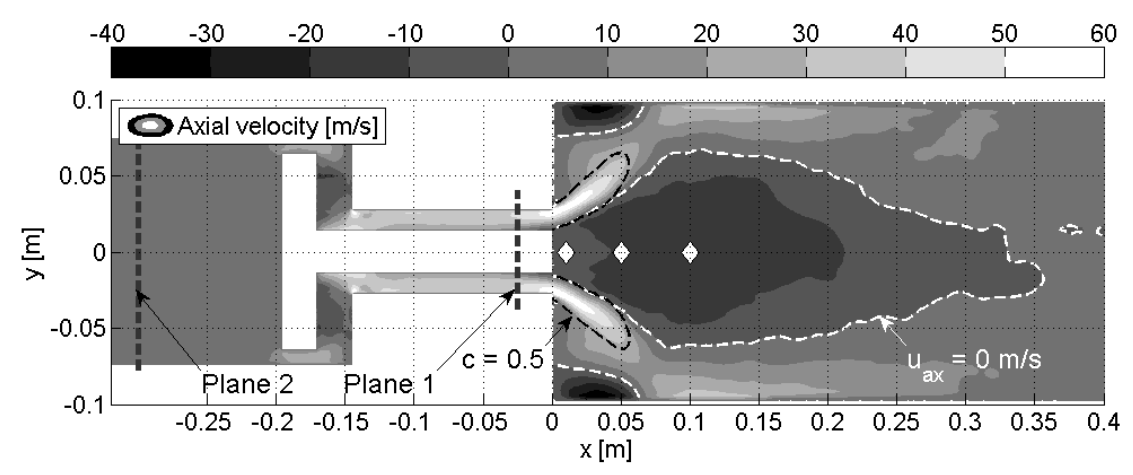

(a) Sampling planes upstream and downstream of the swirler.

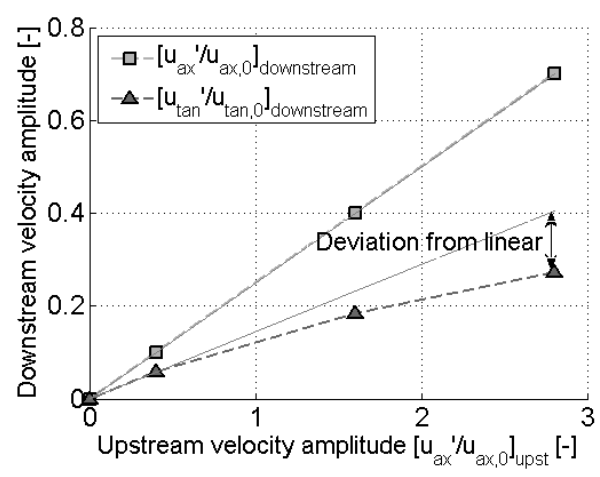

(b) Axial and tangential velocity fluctuations downstream of the swirler (plane 1 in Fig. 4.14(a)) versus axial fluctuations upstream of the swirler (plane 2)

Figure 4.14: Conversion of axial fluctuations upstream of the swirler to axial $\left(u_{a x}^{\prime}\right)$ and tangential fluctuations $\left(u_{t a n}^{\prime}\right)$ downstream of the swirler. 
becomes relatively smaller than the amplitude of the axial fluctuations. This can best be seen by comparing the top plot $\left(u^{\prime} / u_{0}=0.1\right)$ and bottom plot $\left(u^{\prime} / u_{0}=0.7\right)$. This indicates that the swirler transmits axial and tangential fluctuations differently. In figure 4.14(b) the normalized amplitude of axial and tangential fluctuations downstream of the swirler (plane 1 in figure 4.14(a)) is shown as a function of axial fluctuations upstream of the swirler (plane 2 in figure 4.14(a)). It can be seen that the downstream amplitude is significantly lower than the upstream excitation amplitude (as discussed in section 4.5), meaning that part of the excitation is reflected or dissipated in the swirler. It can be seen that the swirler transmits axial fluctuations linearly, but the propagation of tangential fluctuations is a function of the excitation amplitude. This means that also the transfer function of the swirler is nonlinearly dependent on the amplitude of axial fluctuations upstream of the swirler.

A remarkable result is found in figure 4.15 by plotting the flame surface fluctuations not only as a function of the axial velocity component, but also as a function of the tangential fluctuation, taken at reference plane 1 in the premixing passage (see figure 4.14(a)). Similar to the data shown in figure 4.12, the flame surface fluctuations are nonlinearly dependent on the axial excitation amplitude. However, the flame surface fluctuations depend almost linearly on the tangential component.

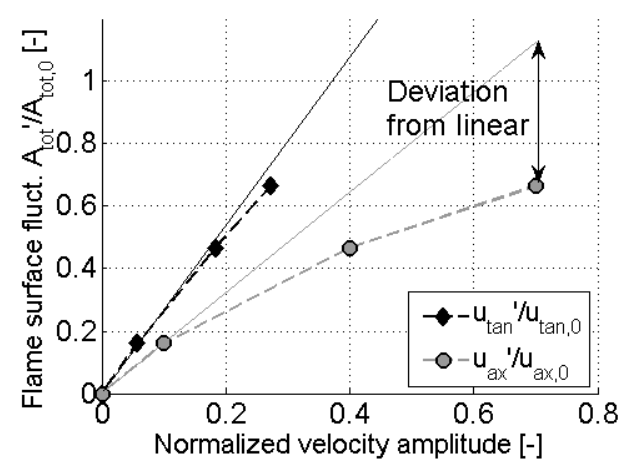

Figure 4.15: Flame surface fluctuations as a function of axial fluctuations (grey line) and as a function of tangential velocity fluctuations (black line).

The nonlinear dependence on the axial velocity component can be explained by the fact that this component also generates a fluctuating volume flow of burnable mixture that somehow needs to be consumed by the flame. The tangential velocity fluctuations on the other hand do not generate a fluctuation of burning material. The linear relation between heat release fluctuation and tangential velocity fluctuation is a result of the nonlinear coupling between the fluctuations of the two velocity components produced by the swirler (see figure 4.14(b)).

A more detailed investigation of the contribution of the different velocity components and the role of the swirler to flame saturation would require an independent variation of the different velocity components. Designing an experiment or simulation in which the axial and tangential velocity can be independently excited would be an interesting next step. 


\subsection{Nonlinear stability analysis with the Generalized Instability Model}

In this section the Flame Describing Function, predicted with LES in section 4.5, is used to predict the limit cycle pressure amplitude with the Generalized Instability Model. The model setup is described in subsection 4.7.1 and in subsection 4.7.2 the results are compared to experimental data.

\subsubsection{Model setup}

In figure 4.16(a) it is schematically shown how the acoustic waves propagate through the radial swirler. Schimek et al. [85,86] found in their measurements that the swirler cannot be considered acoustically transparent. This means that the swirler has an influence on the acoustic mode shapes and for that reason has to be modeled in thermo-acoustic codes.
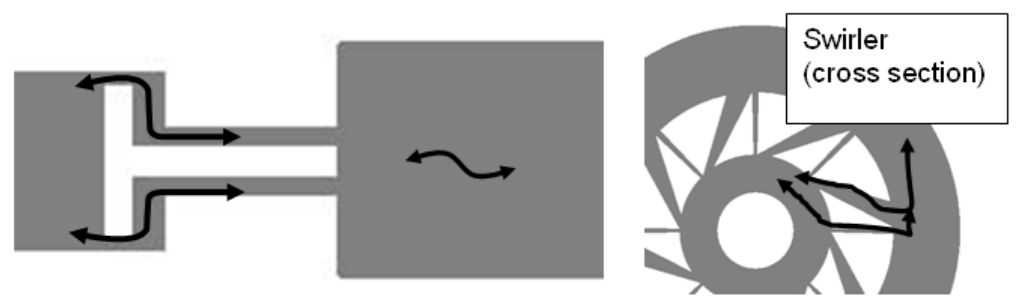

(a) Acoustic wave propagation through the swirler.

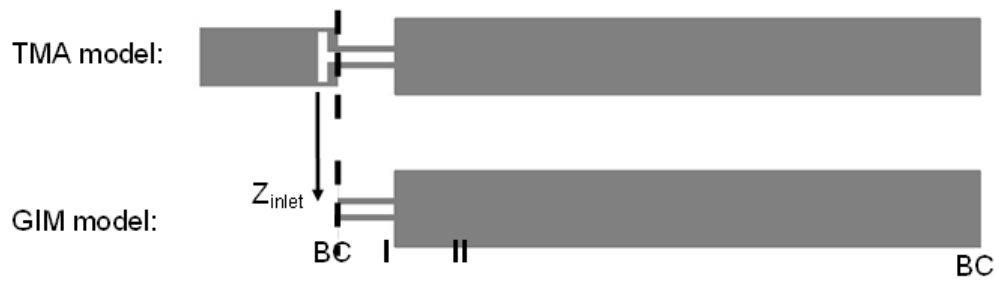

(b) Domain selection for the GIM model. The impedance boundary condition at the inlet of the GIM model $\left(Z_{\text {inlet }}\right)$ has been determined using a TMA model that includes the swirler. See Appendix $\mathrm{H}$ for the procedure.

Figure 4.16: Domain for the nonlinear stability analysis.

Modeling this particular swirler would require the use of both branching / conjunction and diffuser elements. Branching / conjunction elements split / merge the acoustic path into different sub paths, whereas diffuser elements model a duct with a non constant cross-section. The Generalized Instability Model does not have these capabilities.

Therefore, the GIM domain is such that it starts immediately downstream of the swirler, at the inlet to the annular duct (see figure 4.16(b)). Because the inlet boundary condition of the GIM model was not known beforehand, a different thermo- 
acoustic design tool, the Transfer Matrix Approach (TMA), has been used to determine the impedance and reflection coefficient at this location. The Transfer Matrix Approach is a frequency domain network model. General information about TMA is given in Appendix A. The TMA model for this configuration, the acoustic mode shapes calculated with TMA and more information about the procedure used to calculate the impedance downstream of the swirler can be found in Appendix $\mathrm{H}$.

As can be seen in figure 4.16(b), the GIM model consists of 2 domains: the annular duct (I) and the combustion chamber (II). The mean properties for these domains are tabulated in table 4.6 .

\begin{tabular}{lcc}
\hline $\begin{array}{l}\text { Reflection } \\
\text { coefficient }\end{array}$ & $\begin{array}{c}\text { Domain I } \\
\text { (from Appendix H) }\end{array}$ & $\begin{array}{c}R_{\text {outlet }}=-0.99 \\
\text { (measured) }\end{array}$ \\
\hline Length & $0.171 \mathrm{~m}$ & $1.5 \mathrm{~m}$ \\
\hline Area & $0.00178 \mathrm{~m}^{2}$ & $0.0314 \mathrm{~m}^{2}$ or $0.00866 \mathrm{~m}^{2}$ \\
\hline Mean velocity & $u_{0}=33.4 \mathrm{~m} / \mathrm{s}$ & $u_{0}=7.5 \mathrm{~m} / \mathrm{s}$ or $u_{0}=23.8 \mathrm{~m} / \mathrm{s}$ \\
\hline Sound speed & $c=445 \mathrm{~m} / \mathrm{s}$ & $c=790 \mathrm{~m} / \mathrm{s}$ \\
\hline Pressure & $p_{0}=1 \mathrm{bar}$ & $p_{0}=1 \mathrm{bar}$ \\
\hline Temperature & $T_{0}=493 \mathrm{~K}$ & $T_{0}=1620 \mathrm{~K}$ \\
\hline
\end{tabular}

Table 4.6: Mean properties and boundary conditions used for the different domains.

The temperature presented in table 4.6 is the mean temperature between inlet and outlet of the combustion chamber, determined from the CFD simulation (see figure 4.17).

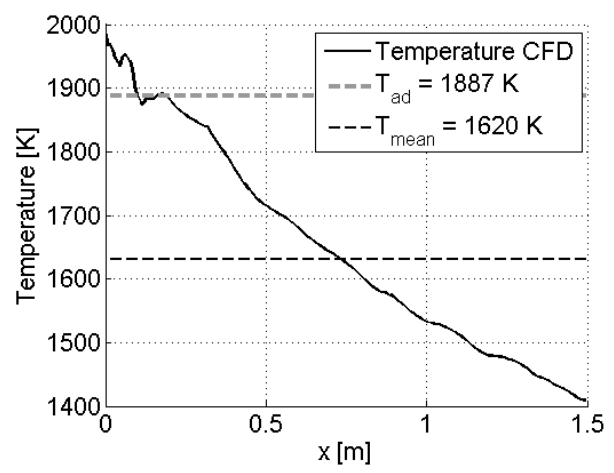

Figure 4.17: CFD Temperature distribution for the partially premixed case. The mean temperature used in GIM $\left(T_{\text {mean }}\right)$ and the adiabatic flame temperature $T_{a d}$ (based on the mean equivalence ratio) are indicated as well.

The heat release source was located at the inlet of the combustion chamber and is modeled as a point source. This can be done, because the length of the flame is small 
compared to the acoustic wave length $\left(L_{\text {flame }}<<\lambda\right)$, see Ref. [49]. Simulations with both the simulated and measured Flame Describing Functions have been performed, both for the perfectly premixed and partially premixed case. The measured FDF is directly based on $O H^{*}$ chemiluminescence (see equation 4.1).

Since the FDF was only known at discrete frequencies and amplitudes, the values had to be interpolated and extrapolated for the whole frequency and amplitude range. The gain was linearly interpolated between different frequencies. The FDF as a function of the excitation amplitude was interpolated using an exponential curve fit (see figure 4.18). For some cases, the acoustic velocity became even larger than the range for which data on the FDF was available. In these cases, the exponential curve fit was used to extrapolate the gain.

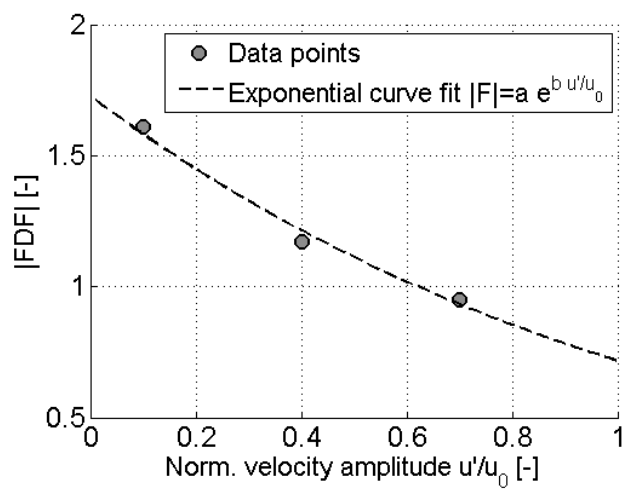

Figure 4.18: Interpolation of the FDF as a function of excitation amplitude. Graphs shows an example for the the perfectly premixed case, using the simulation data at $115 \mathrm{~Hz}$.

\subsubsection{Results}

\section{Partially premixed case}

In figure 4.19 the results of the nonlinear stability analysis for the partially premixed case are shown. First the acoustic pressure as a function of time is compared for GIM simulations with the measured (figure 4.19(a)) and simulated (figure 4.19(b)) Flame Describing Function. It can be seen that a smaller limit cycle pressure amplitude is predicted when the simulated FDF is used, which can be explained by the smaller gain of the simulated FDF (see figure 4.8(a)). With the measured FDF, the limit cycle is reached after approximately 0.4 seconds, whereas with the simulated FDF, the limit cycle has already established after 0.1 seconds simulation time. This can be explained as follows: because of the smaller gain of the simulated FDF, the gain and losses compensate each other sooner. After $0.1 \mathrm{~s}$, the growth rate for the simulation with the simulated FDF becomes zero. With the measured FDF, the growth rate may be small, but does not vanish completely between 0.1 and $0.4 \mathrm{~s}$, resulting in a slow increase of the pressure amplitude.

A balance diagram of the energy gain and losses is shown in figure 4.19(c), cal- 


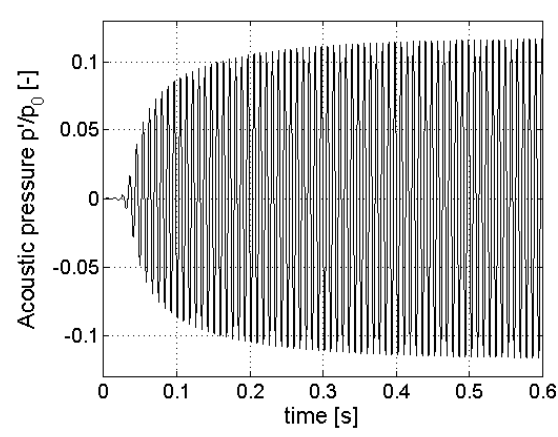

(a) Limit cycle prediction with GIM for the partially premixed case, using the measured FDF.

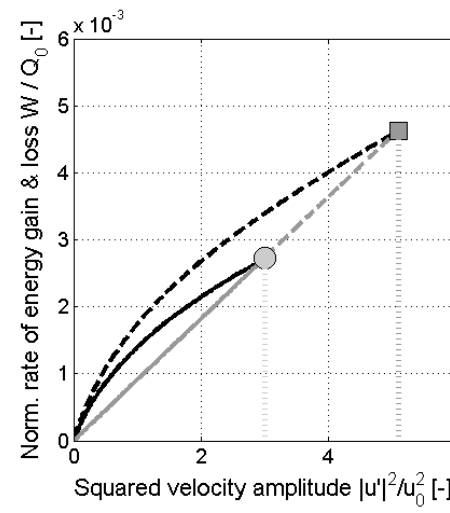

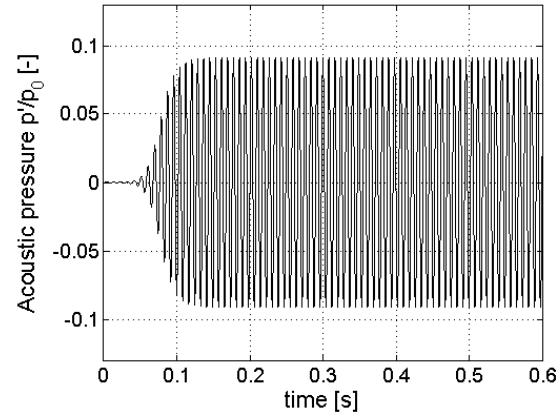

(b) Limit cycle prediction with GIM for the partially premixed case, using the simulated FDF.

(c) Energy balance of gain and losses for the partially premixed case, using the measured and simulated FDF.

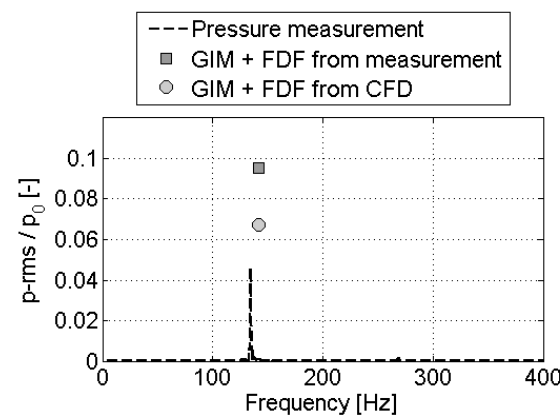

(d) Comparison of dynamic pressure spectrum obtained by measurements and GIM simulations with both the measured and simulated Flame Describing Function - linear scale.

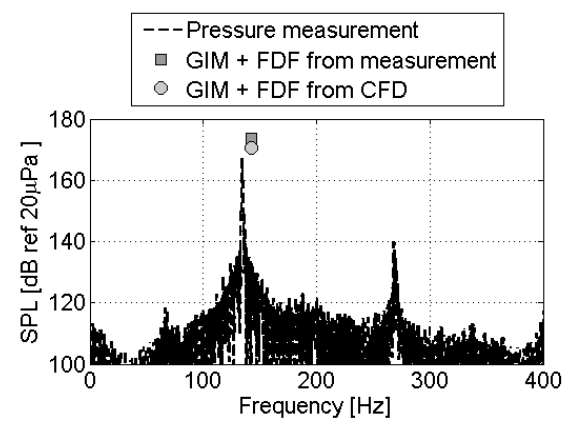

(e) Comparison of dynamic pressure spectrum obtained by measurements and GIM simulations with both the measured and simulated Flame Describing Function - SPL scale.

Figure 4.19: Results of the nonlinear stability analysis for the partially premixed case. 
culated as a function of the squared acoustic velocity amplitude. Both $W_{\text {gain }}$ and $W_{\text {loss }}$ have been normalized by the mean heat release $Q_{0}$. Expressions for $W_{\text {gain }}$ and $W_{\text {loss }}$ can be found in Chapter 2, see equations 2.24 and 2.25. The energy balance of figure 4.19(c) looks similar to the sketch in figure 1.4. It can be seen that the losses are identical for both cases, but the slope of the gain based on the measured FDF is larger, resulting in a larger limit cycle pressure amplitude. About $0.26 \%$ to $0.46 \%$ of the total heat released by the flame is converted into acoustic energy.

In figure 4.19(d) and 4.19(e) the predicted frequency and root mean square acoustic pressure are compared against experiments. The values are tabulated as well in table 4.7. It can be seen that the prediction with the simulated FDF leads to a pressure amplitude that is closer to the measurements than the prediction using the measured FDF. This can be explained by the fact that the simulated FDF is actually defined using the heat release fluctuations $Q$, which are not linearly proportional to $O H^{*}$ fluctuations. However, also with the simulated FDF the amplitude is a bit over predicted $(+46 \%)$. It should be noted that on a logarithmic SPL scale the overprediction is only $3.3 \mathrm{~dB}$, which is good enough for practical applications.*

The frequency from the simulation $(143 \mathrm{~Hz})$ is close to the measurements (135 $\mathrm{Hz}$ ). The difference of $8 \mathrm{~Hz}$ could be caused by the fact that a constant temperature of $1620 \mathrm{~K}$ was applied in the GIM model (see table 4.6). In reality the temperature varies approximately linearly over the combustion chamber (see figure 4.17), but this can not be modeled with GIM. However, the frequency is considered to be reasonably close to the measured value. ${ }^{\dagger}$

As a final note it should be mentioned that for the second eigen mode of the combustion chamber $(f \approx 364 \mathrm{~Hz}$ ) a growth rate of $\Gamma=-20 \mathrm{rad} / \mathrm{s}$ was found, indicating a stable mode. The gain of the FDF was linearly extrapolated for this frequency, because only data up to $323 \mathrm{~Hz}$ was available. This prediction is consistent with the experiments, since no instabilities at the second eigenmode have been observed.

\section{Perfectly premixed case}

The results for the perfectly premixed case can also be found in table 4.7. The perfectly premixed case did not show any self-excited instabilities in the measurements. The GIM simulation with the measured FDF was stable as well, which is consistent with these findings. The GIM simulation with the simulated FDF on the other hand resulted in an instability with $p_{r m s} / p_{0}=0.021$. This is a relatively small and possibly harmless pressure amplitude, but the reason for this overprediction should nonetheless be investigated. This result may be caused by the discrepancy in phase of the FDF between measurements and simulations (see figure 4.7(b) and figure 4.9(b)). Therefore, the sensitivity of the predicted limit cycle amplitude on the phase of the FDF was investigated. This analysis will be discussed in the following paragraph.

${ }^{*}$ The sound pressure level (SPL) is defined as:

$$
S P L=20 \log \left(p / p_{\text {ref }}\right) \quad \text { with } \quad p_{\text {ref }}=20 \mu P a
$$

\footnotetext{
† In an earlier version of the GIM model, the temperature in the whole combustion chamber was assumed to be the adiabatic flame temperature $\left(T_{a d}=1887 \mathrm{~K}\right)$. This resulted in a frequency of $157 \mathrm{~Hz}$ $(+14 \mathrm{~Hz})$.
} 


\begin{tabular}{lcc}
\hline & Frequency & Pressure amplitude \\
\hline Meas.: Perfectly premixed & - & $p_{r m s} / p_{0} \approx 0$ \\
\hline Meas.: Partially premixed & $135 \mathrm{~Hz}$ & $p_{r m s} / p_{0}=0.046$ \\
\hline $\begin{array}{l}\text { GIM: Perfectly premixed } \\
\text { with measured FDF }\end{array}$ & - & $p_{r m s} / p_{0}=0$ \\
\hline $\begin{array}{l}\text { GIM: Perfectly premixed } \\
\text { with simulated FDF }\end{array}$ & $141 \mathrm{~Hz}$ & $p_{r m s} / p_{0}=0.021$ \\
\hline $\begin{array}{l}\text { GIM: Partially premixed } \\
\text { with measured FDF }\end{array}$ & $\begin{array}{c}143 \mathrm{~Hz} \\
(+6 \%)\end{array}$ & $\begin{array}{c}p_{r m s} / p_{0}=0.095 \\
(+106 \% /+6.3 \mathrm{~dB})\end{array}$ \\
\hline $\begin{array}{l}\text { GIM: Partially premixed } \\
\text { with simulated FDF }\end{array}$ & $\begin{array}{c}142 \mathrm{~Hz} \\
(+5 \%)\end{array}$ & $\begin{array}{c}p_{r m s} / p_{0}=0.067 \\
(+46 \% /+3.3 \mathrm{~dB})\end{array}$ \\
\hline $\begin{array}{l}\text { Self-excited CFD } \\
\text { simulation }\end{array}$ & $\begin{array}{c}138 \mathrm{~Hz} \\
(+1 \%)\end{array}$ & $\begin{array}{c}p_{r m s} / p_{0}=0.034 \\
(-26 \% /-2.6 \mathrm{~dB})\end{array}$ \\
\hline
\end{tabular}

Table 4.7: Comparison of frequency and dynamic pressure amplitude between measurements (Meas.) and GIM simulations. For the partially premixed case, the off-set related to the measurements is given in parentheses.

\section{Sensitivity Analysis}

In section 2.3 .1 of Chapter 2 it was already shown that the predicted limit cycle pressure amplitude is quite sensitive on the phase of the Flame Describing Function. The influence of the phase will now be investigated for the current test case as well. The gain of the FDF has not been varied in this study and corresponds to the gain of the FDF at the first eigen mode of the combustor (approximately $142 \mathrm{~Hz}$ ). The sensitivity analysis was done both for the partially premixed and perfectly premixed case.

In figure 4.20 the predicted limit cycle pressure amplitude as a function of the phase is shown. The markers represent the baseline results. The pressure amplitude $p_{r m s} / p_{0}$ for these baseline results corresponds to the values shown in table 4.7, whereas the phase $\angle \mathcal{F}$ for these baseline results corresponds to the phase of the Flame Describing Functions in figure 4.7(b) and 4.8(c). For instance, the data point "GIM: Part. premixed with simulated FDF - baseline result", represented by the gray circle, has a phase of approximately $-1.1 \pi$, which is also the phase of the FDF at 142 $\mathrm{Hz}$ in figure 4.8(c).

The 4 curves correspond to phase sweeps for the different cases. It should be stressed that the frequency of oscillation did not significantly change, it is always the first eigen mode of the combustor that is considered in this graph. It can be seen that the burner is most stable at a phase of $\angle \mathcal{F}=-1.5 \pi$, and most unstable at $\angle \mathcal{F}=-0.5 \pi$ or $\angle \mathcal{F}=-2.5 \pi$. It can also be seen that the measurement of the partially premixed case (grey asterisk) was not taken at the potential most unstable condition. This means that if the time delay between heat release and pressure fluctuations would be decreased in experiments (which can be done for instance by changing the equivalence ratio or air preheat temperature), a potential larger pressure amplitude 


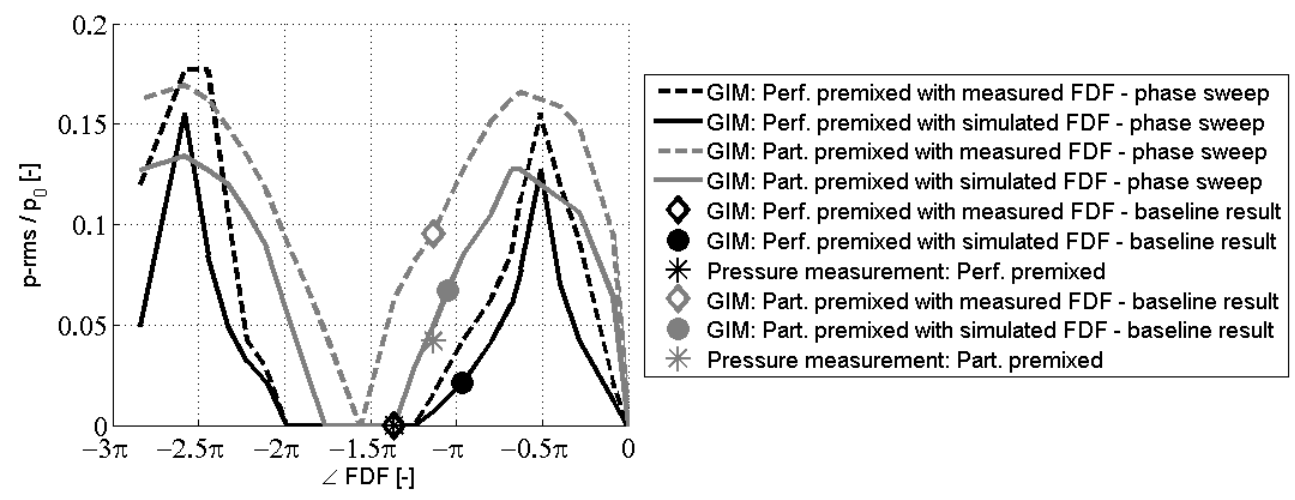

Figure 4.20: Predicted limit cycle pressure amplitude as a function of the phase $(\angle \mathcal{F}=$ $-2 \pi f \tau)$. The asterisks show the pressure measurements and the diamonds and circles show the GIM results according to the baseline values of the phase. The baseline values correspond to the values shown in figure 4.7(b) and 4.8(c), interpolated at a frequency of $142 \mathrm{~Hz}$. The four curves show the results of the sensitivity analysis.

would be found.

Comparing the two black markers of the perfectly premixed case, the results clearly show that the reason that a pressure amplitude of $p_{r m s} / p_{0}=0.021$ was found for the perfectly premixed with simulated FDF can be attributed to the under prediction of the phase of the FDF. The offset in phase between the measured and simulated FDF of the partially premixed case was much smaller, which explains that a better prediction was obtained for the partially premixed case. This again shows how important it is to predict the phase of the FDF correctly. For this case, it would be recommended to review the CFD setup and combustion model, in order to find out if an improvement of the phase of the simulated FDF for the perfectly premixed case is possible. It should be noted however that an almost identical phase was found with AVBP, using a completely different combustion model (see Appendix F, figure F.11(b)).

Finally, the results of the sensitivity analysis can be used to investigate the relation between the predicted linear growth rate $\Gamma$ for small pressure amplitudes and the final predicted limit cycle pressure amplitude. Initially, for small pressure amplitudes, the pressure increases exponentially according to $p^{\prime}=p_{\text {init }} \cos (\omega t) \exp \left(\frac{\Gamma}{2 \pi} t\right)$. All GIM simulations were started at $p_{\text {init }} / p_{0}=10^{-5}$. The linear growth rate was determined at this initial pressure amplitude and was varied by changing the phase $\angle \mathcal{F}$ as described above.

It can be seen in figure 4.21 that the initial linear growth rate and final limit cycle pressure amplitude are highly correlated. For negative growth rates, a zero limit cycle pressure amplitude is found, as expected. In the range $50<\Gamma<120 \mathrm{rad} / \mathrm{s}$, the pressure amplitude increases approximately linearly with growth rate. It can also be seen that the curves for the partially premixed and perfectly premixed case coincide. For $\Gamma>120 \mathrm{rad} / \mathrm{s}$, the curves do not coincide anymore but rather show some spread in the data. However, also in this region the limit cycle pressure amplitude 


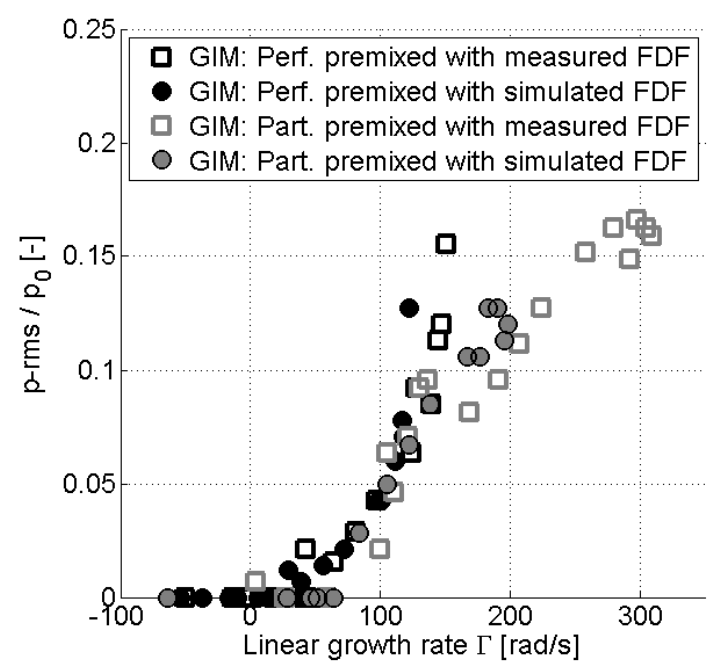

Figure 4.21: Predicted limit cycle pressure amplitude as a function of the predicted linear growth rate $\Gamma$. The growth rate was determined at an initial condition of $p_{\text {init }} / p_{0}=10^{-5}$.

keeps increasing for larger growth rates. This is a useful result. It means that if the limit cycle pressure amplitude and linear growth rate for a certain operating point are known, the limit cycle pressure amplitude for a different operating point can be estimated by just looking at the change of the linear growth rate.

\subsection{Self-excited simulation}

As discussed in Chapter 1 (see figure 1.3), another method to study thermo-acoustic instabilities is the self-excited method, in which the CFD simulation becomes unstable by itself and no separate forcing at the boundaries is required. For completion, this method was also investigated for the partially premixed case.

In order to obtain a self-excited instability, acoustic reflection at the boundaries is required. Therefore the time scalar at the inlet and outlet have been set to a small value ( $\tau=3 \cdot 10^{-5} \mathrm{~s}$, see table 4.3). This time scalar leads to a reflection coefficient at the outlet of approximately $R_{\text {outlet }}=-0.99$ at a frequency of $142 \mathrm{~Hz}$ (the reflection coefficient as function of $f$ and $\tau$ was shown in Chapter 3, figure 3.4). This means that the non-reflective boundary condition is used to mimic an impedance boundary condition, so that the losses at the outlet in the self-excited simulation are more or less equal to the losses in the GIM simulations presented in the previous section.*

The initial solution of the self-excited simulation was identical to the initial solution for the simulations with excitation (to obtain the Flame Describing Function), which does show show significant pressure fluctuations.

*The CFD domain still starts $30 \mathrm{~cm}$ upstream of the swirler, which is not the physical location of the inlet boundary condition. However, as shown in table H.1 of Appendix H, the geometry of the upstream duct has negligible effect on the acoustic modes in the combustion chamber, justifying this simplification. 
The results of the self-excited simulation can be found in figure 4.22. First the normalized pressure at the inlet of the combustion chamber can be seen in figure 4.22(a). The pressure amplitude immediately starts to grow after the start of the simulation. A constant pressure amplitude of $p^{\prime} / p_{0} \approx 0.046$ is obtained after about 0.1 second simulation time (approximately 13 periods). The amplitude of the velocity and heat release fluctuations converges to a constant value as well (see figure 4.22(c)).

A zoom up of the pressure can be seen in figure 4.22(b). In this figure the measured pressure, obtained from a pressure transducer located at inlet of the combustion chamber, can be seen as well. The frequency of the instability agrees well. The pressure amplitude from the measurement was slightly larger however.

The normalized flame source term $W_{\text {gain }}$ (see equation 2.24) can be seen in figure $4.22(\mathrm{~d})$. The flame source term also converges to a constant value after 0.1 second of simulation.

In figure 4.22(e) and 4.22(f) a spectrum of the result can be found. Only a single data point for the self-excited simulation is shown, because the time series were not long enough to show a continuous pressure spectrum as function of frequency. The exact values of the pressure amplitude can also be found in table 4.7. The results from the previous section, obtained with GIM are shown as well. The frequency and amplitude obtained with the self-excited method is slightly closer to the experimentally found values than the GIM results.

In figure 4.23 contour plots of the rms fields can be seen. The axial pressure, velocity and heat release distribution are shown in figure 4.23(a). It can be seen that the configuration has a pressure node at the outlet $(x=1.5 \mathrm{~m})$. The maximum pressure amplitude is found in the annular duct between the swirler and the combustion chamber. Furthermore, the configuration has a velocity anti-node at the outlet of the combustion chamber. The pressure mode shape from the self-excited simulation was also compared against the pressure mode shape from the GIM model in figure 4.24. Except from some differences close to the swirler, which would be expected, the mode shapes match well.

A close-up of the root mean square heat release fluctuation can be found in 4.23(b). It can be seen that the heat release mainly fluctuates in the shear layer at the inner recirculation zone. The root mean square heat release in the shear layer at the outer recirculation zone is negligible, meaning that the heat release here does not fluctuate much. Finally the local phase difference between heat release fluctuations and velocity fluctuations upstream of the flame is shown in figure 4.23(c). The black line corresponds to a mean phase difference of $\angle=-0.78 \pi$, which is the phase difference between the volume integrated heat release and velocity fluctuations in figure 4.22(c). This value can be considered as some sort of average phase difference over the flame. It can be seen that the phase at the inner recirculation zone is smaller than the phase at the outer recirculation zone, which indicates that a certain disturbance upstream of the flame reaches the inner shear layer faster than the outer shear layer. 


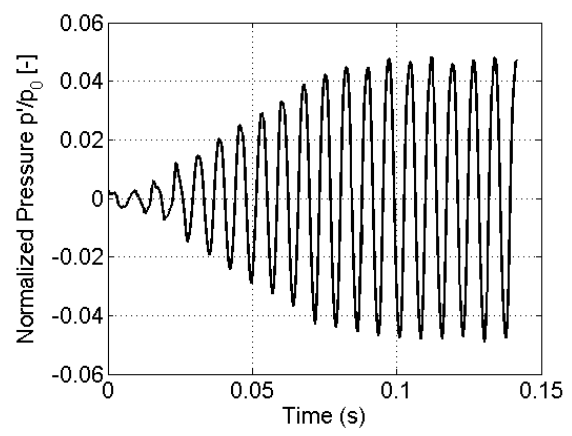

(a) Normalized dynamic pressure.

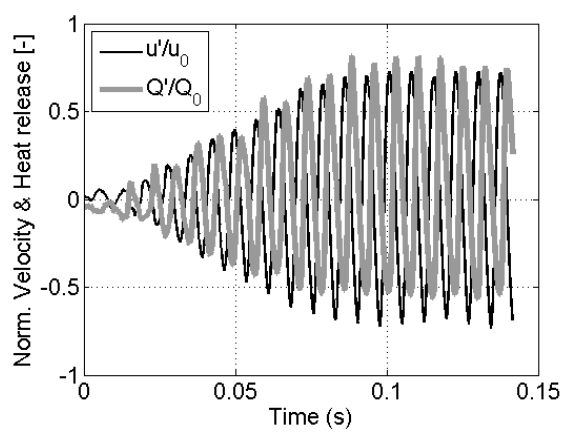

(c) Normalized velocity and heat release fluctuations.

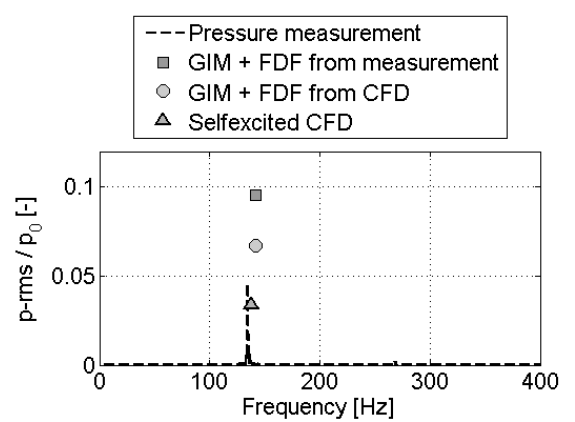

(e) Comparison of dynamic pressure spectrum from self-excited simulation with measurements and GIM simulations in frequency domain - Linear scale.

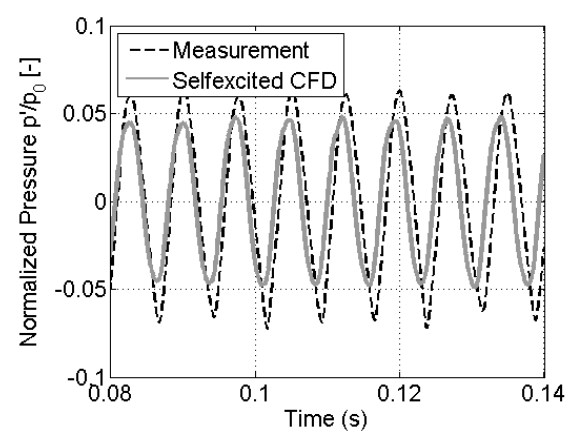

(b) Zoom in on figure 4.22(a) and comparison of dynamic pressure with measured time signals.

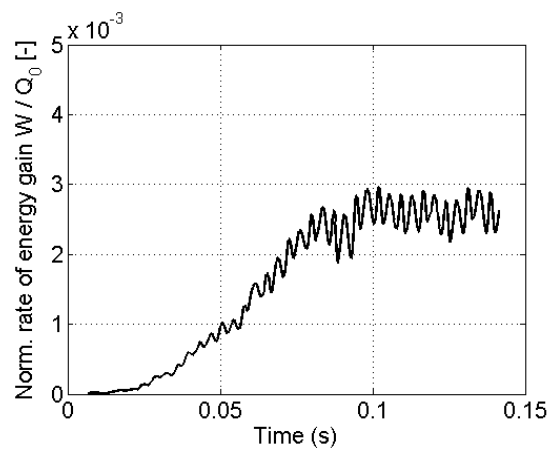

(d) Normalized rate of acoustic energy production.

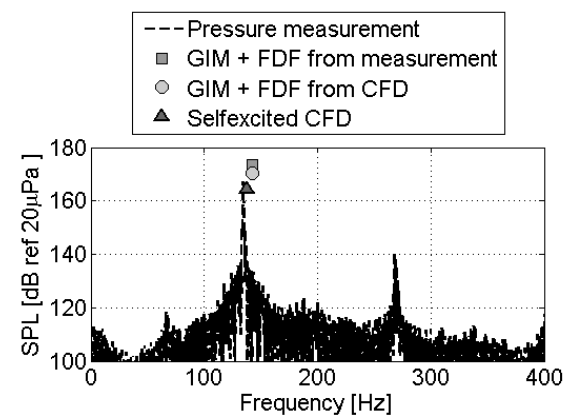

(f) Comparison of dynamic pressure spectrum from self-excited simulation with measurements and GIM simulations in frequency domain - SPL scale.

Figure 4.22: Results of the self-excited simulation. 

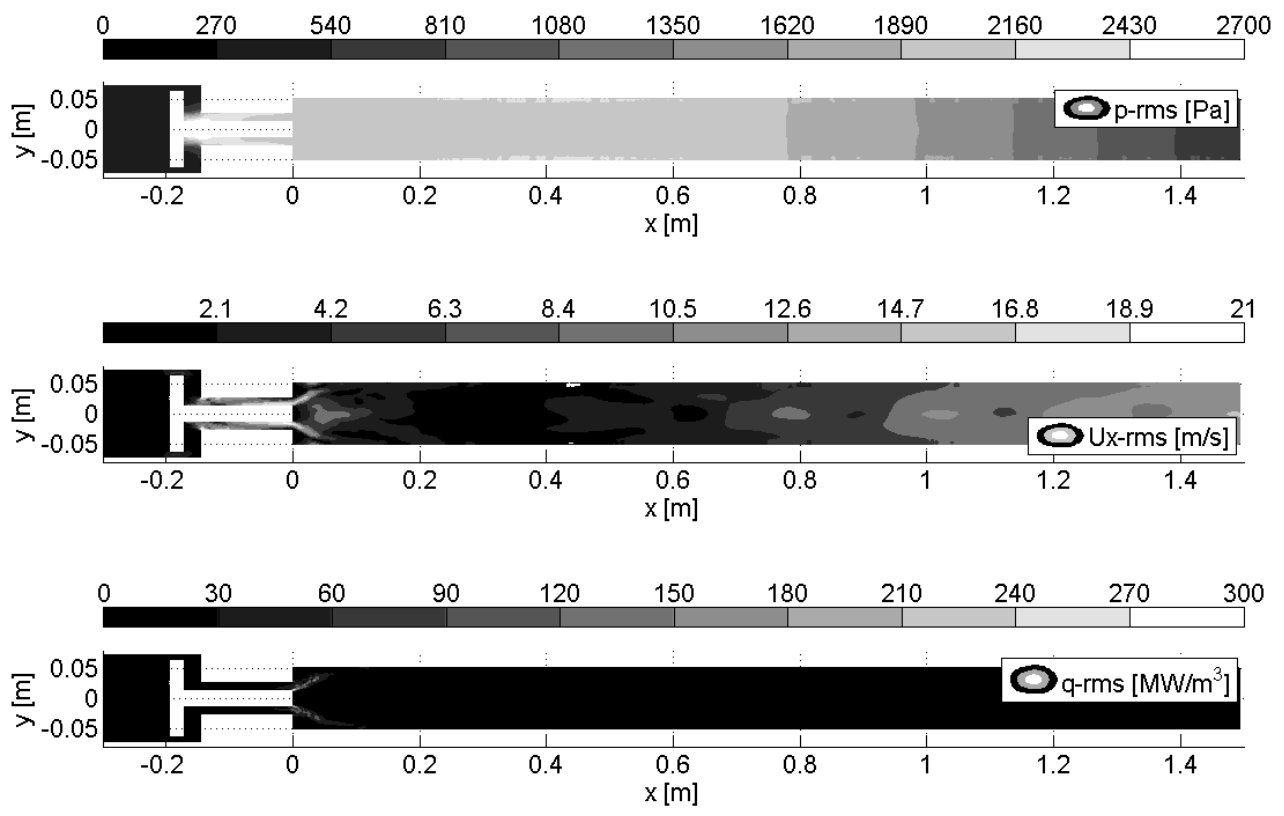

(a) Magnitude of pressure (top), velocity (middle) and heat release density (bottom).

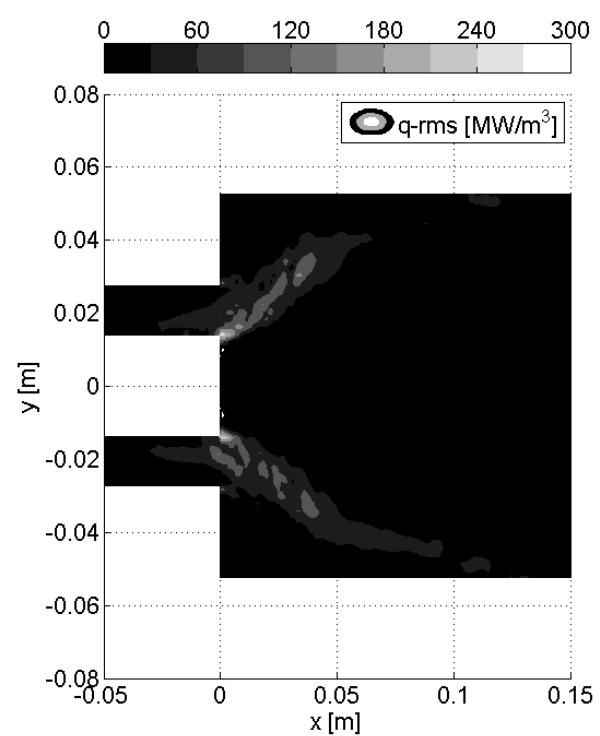

(b) Magnitude of heat release density (zoomed).

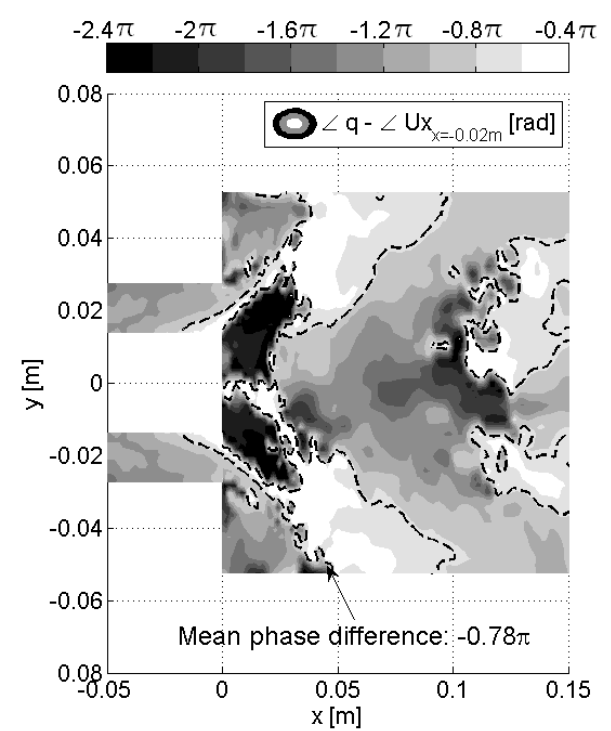

(c) Phase between heat release density and velocity at reference position $\mathrm{x}=-0.02 \mathrm{~m}$ (corresponding to the sampling plane in fig. 4.4). Dashed line indicates mean phase, obtained using the integrated quantities in fig. 4.22(c).

Figure 4.23: Fourier transformed pressure, velocity and heat release fields at a frequency of $135 \mathrm{~Hz}$. 


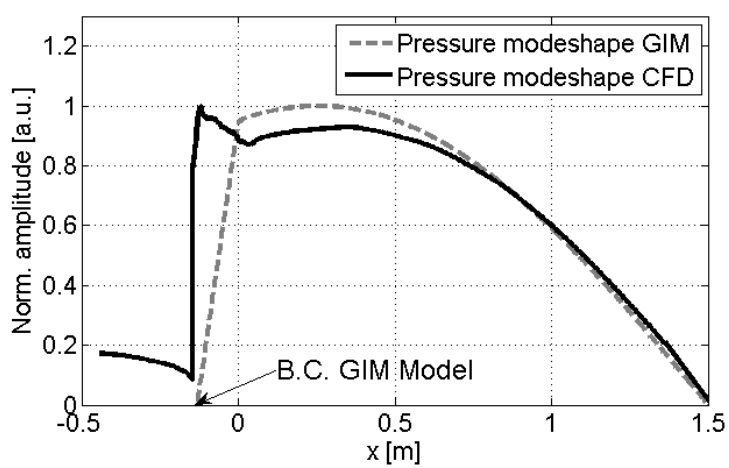

Figure 4.24: Comparison of the pressure mode shape obtained from the self-excited CFD simulation with the mode shape from GIM. Both mode shapes have been normalized by their maximum values.

\subsubsection{Comparison with forced response approach}

As mentioned above, the self-excited simulation shows a slightly better comparison to experimental data than the forced response method. This is somehow expected, because not only the flow field, but also the acoustics are modeled with a 3D code. If only one design or operating point is of interest for a certain configuration, the selfexcited method is cheaper with respect to CPU time as well. As can be seen in figure 4.22(a), about $140 \mathrm{~ms}$ of simulation time was required for the self-excited simulation, whereas 9 runs of about $80 \mathrm{~ms}$ simulation time each was required to obtain the Flame Describing Function (see table 4.5).

The main advantage of the forced response method, however, is the fact that it directly allows for parameter sweeps. It can for instance be estimated in which direction the flame time lag (and thus the phase of the FDF) changes when the operating point (e.g. equivalence ratio or air preheat temperature) is changed. A stability map as function of the phase of the FDF, such as shown in figure 4.20, can be created within a couple of hours. Also the effect of certain design changes (e.g. a change in combustion chamber length) can be easily assessed. Such variation studies are much more expensive with the self-excited method, since for each operating point a new CFD simulation would be required.

Which approach is most suitable for a certain configuration therefore depends on the question how many operating points or design changes need to be tested.

\subsection{Conclusions}

In this chapter it was shown that the Flame Describing Function of a premixed swirl flame can be successfully predicted using Large Eddy Simulations. Both a perfectly premixed and a partially premixed operating point were studied. The saturation of the heat release of the flame at a frequency of $115 \mathrm{~Hz}$, which was observed during 
measurements, was correctly captured. At higher frequencies (above $200 \mathrm{~Hz}$ ), no noticeable saturation was found in either the experiments or simulations.

A detailed investigation of the data for the perfectly premixed case revealed that the flame saturates due to a nonlinear increase of the flame surface with the amplitude of the velocity fluctuations. From an investigation of the different components of the flame surface (resolved, unresolved and total flame surface), it was concluded that the major part of the saturation can be attributed to saturation of the Flame Surface Density $|\nabla c|$.

The FDF from the CFD simulations was used together with the Generalized Instability Model to predict the limit cycle pressure amplitude. A good comparison between numerical and experimental data was found for the partially premixed case. The frequency was predicted within $8 \mathrm{~Hz}$, the pressure amplitude within $46 \%$ (3.3 $\mathrm{dB})$.

For the perfectly premixed case, an instability was found as well however. This instability was not observed in experiments. This instability only occurred when the FDF from the CFD simulations was used in the Generalized Instability Model. GIM simulations with the measured FDF also predicted stable operation. A sensitivity analysis on the phase of the FDF led to the conclusion that the under prediction of the phase in the simulations is the root cause for this result. For this case, it would be recommended to review the CFD setup and combustion model, in order to find out if an improvement of the phase of the simulated FDF for the perfectly premixed case is possible.

Finally a self-excited simulation was performed. A limit cycle was found with only $3 \mathrm{~Hz}$ difference in frequency and $26 \%$ difference in pressure amplitude compared to the experimental data. This means the self-excited simulation is slightly closer to the measurements. Nonetheless, also the results obtained with the forced response method are reasonably good. 


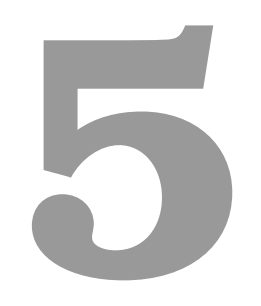

\section{Application to a high pressure multi-jet flame combustor}

\subsection{Introduction}

In this chapter the methods proposed in this work will be applied to a partially premixed industrial combustion system: the DOC burner. The DOC (Depleted Oxygen Combustion) burner is a can-annular type industrial combustion system, which is currently being developed by SIEMENS Energy for future application in gas turbine engines. A description of the DOC burner, as well as a description of the CFD setup, is given in section 5.2.

The key focus in this chapter is on the application of a Multiple Input, Single Output (MISO) flame model. In section 5.3 steady flow field simulation results are presented. In section 5.4 it will be discussed how MISO Flame Describing Functions can be determined from the CFD simulations, which take into account both velocity fluctuations and equivalence ratio fluctuations. In section 5.5 this MISO model is then implemented into a GIM model and the limit cycle pressure amplitude is predicted. Two operating points are studied.

Finally the additional benefits of the Multiple Input, Single Output model compared to a Single Input, Single Output model are discussed. Based on the results of the nonlinear stability analysis using this MISO model, a design change that improves the thermo-acoustic stability of the burner is proposed. 


\subsection{Description of test case and computational domain}

A sketch of the DOC burner (cross-section) can be seen in figure 5.1. The DOC burner features a jet-stabilized combustion zone and consists of 16 jet flames in circumferential direction (indicated with Main flame). Fuel and air are mixed in a premixing passage upstream of the combustion chamber. The fuel is injected perpendicular to the air flow, which is known as the jet-in cross flow principle of mixing [31]. In the center of the combustion chamber, a swirl stabilized flame is located, which serves as a pilot burner. The burnt gases from the swirl flame mix with fresh gases at the base of the jet flame, which helps stabilizing and igniting these main flames. Furthermore a large recirculation zone can be seen in the middle of the combustion chamber.

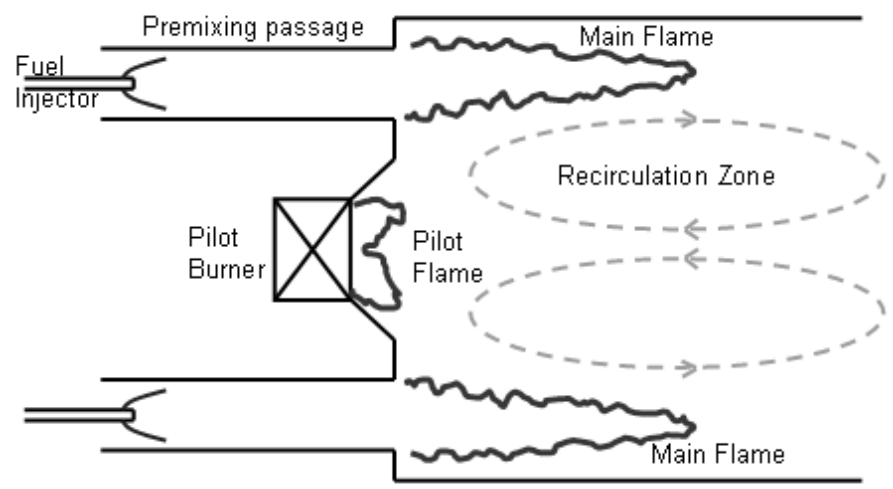

Figure 5.1: Sketch of the DOC burner, combustion chamber geometry and flow field.

In the design stage of a can-annular gas turbine combustion system, a burner is not directly tested in a gas turbine engine. The DOC burner configuration which is discussed in this chapter was tested in a high-pressure test rig. In order to draw useful conclusions about the thermo-acoustic behavior of the burner, it is important that the acoustic boundary conditions of the high pressure rig match the boundary conditions of the engine. A typical arrangement for high pressure testing of an industrial can type combustion system is presented in figure 5.2 [52].

The inlet of the domain is located where the compressor outlet would be in a real engine. The air then flows through a plenum, where it makes a $180^{\circ}$ turn. Finally the air flows through the burner, the combustion chamber, a transition piece, a Vane Simulation Section (VSS), and the exhaust passage. Here the VSS consists of a row of blades that mimics the actual turbine blades in the engine. The outlet of the exhaust rig is choked, providing a well defined acoustic boundary.

In order to predict the thermo-acoustic stability of the DOC burner using the Generalized Instability Model, the whole domain shown in 5.2 needs to be modeled. This is not required however for determining a Flame Describing Function from CFD, see for instance figure 1.3 in Chapter 1. The CFD domain starts upstream of the fuel injectors (see figure 5.1) and ends between the transition piece and the Vane Simulation Section. The computational grid consisted of 16.1 million hexahedral cells. 


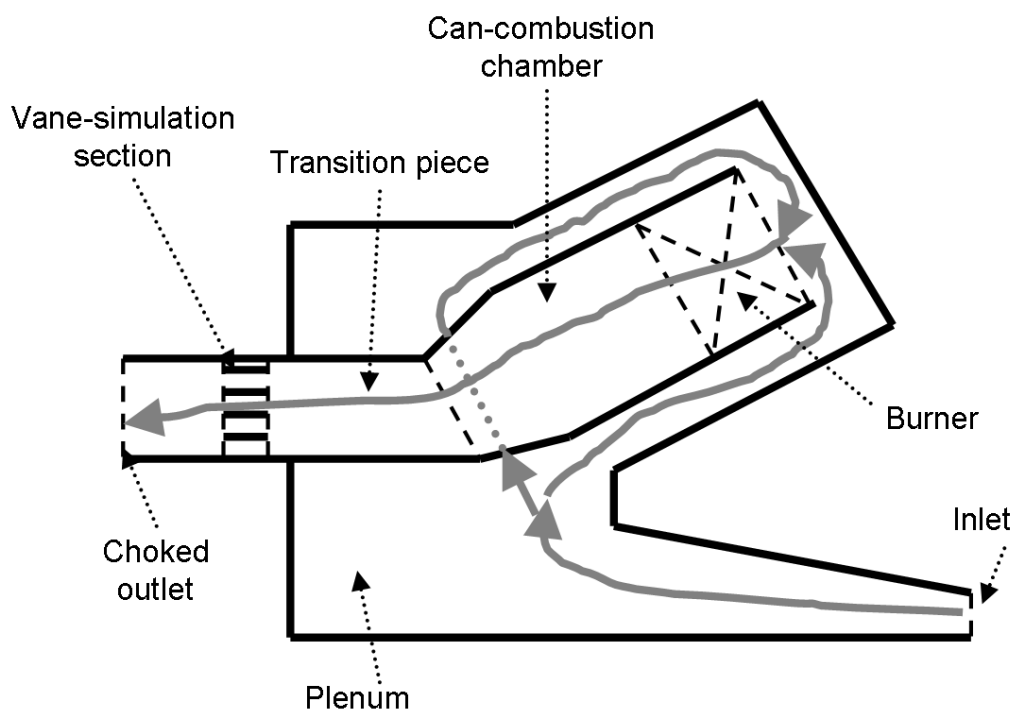

Figure 5.2: Typical computational domain for acoustic simulations in a can-annular combustion system. The grey curve indicates the general flow path. The inlet of the domain is located where the compressor outlet would be in a real engine. The air flows through a plenum, the burner, the combustion chamber, a transition piece, the Vane Simulation Section (VSS), and finally exits the domain through a choked nozzle. The burner section is indicated with a cross, meaning that it can have an arbitrary geometry.

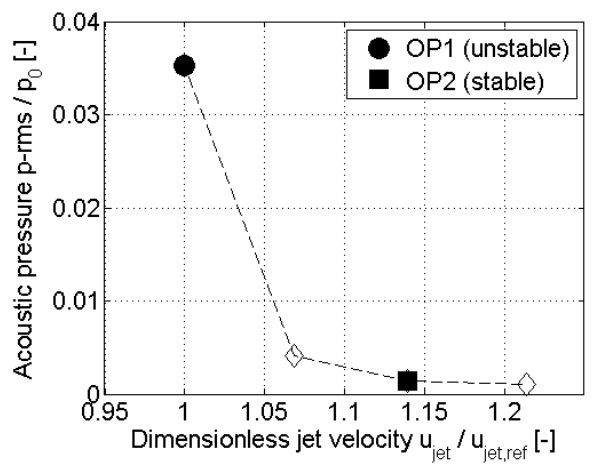

Figure 5.3: Measured acoustic pressure amplitude as function of the jet velocity. The figure shows 4 measured operating points. Two of these operating points ( $O P 1$ and $O P 2)$ have been selected for the CFD analysis. 
The thermo-acoustic stability of the DOC burner is known to be very sensitive on the velocity of the air flowing out of the premixing passage. In figure 5.3 the normalized acoustic pressure as a function of the jet velocity is shown [8]. The jet velocity has been normalized here by a reference velocity $u_{j e t, r e f}$, which corresponds to the jet velocity of operating point $O P 1$ in this figure. It can be seen that $O P 2$ has a $14 \%$ larger velocity and mass flow than $O P 1$, which resulted in stable versus unstable operation. All other operating conditions (e.g. pressure, temperature, overall equivalence ratio) are identical in figure 5.3.

The operating points $O P 1$ and $O P 2$ are studied here by means of CFD analysis. For both operating points first the steady flow field is determined. One acoustic mode appeared to be dominant in the measurements [8]. The dimensionless frequency of this mode was $f^{*}=1.05$, which corresponds to the first longitudinal eigen mode (1L mode).*

Since always only one frequency was found to be excited in measurements, the flame response was only determined at this frequency. As can be seen in table 5.1, in the CFD simulations the flow was excited with three different excitation amplitudes for $O P 1$. For the stable operating poing $O P 2$, the flame response was only determined for $u^{\prime} / u_{\text {jet }}=0.1$.

\begin{tabular}{cc}
\hline OP1 & OP2 \\
\hline Mean flow field & Mean flow field \\
\hline$f^{*}=1.05$ and $u^{\prime} / u_{\text {jet }}=0.10$ & $f^{*}=1.05, u^{\prime} / u_{\text {jet }}=0.10$ \\
$f^{*}=1.05$ and $u^{\prime} / u_{\text {jet }}=0.45$ & \\
$f^{*}=1.05$ and $u^{\prime} / u_{\text {jet }}=0.90$ & \\
\hline
\end{tabular}

Table 5.1: Simulations for the two operating points.

\subsection{Steady flow field results}

For both operating points first the steady flow field was determined. Similar to the swirl stabilized flame from the previous chapter, the steady flow field was determined using non-reflecting boundary conditions. The time scalars at the inlet and outlet were $\tau=0.03 \mathrm{~s}$, corresponding to reflection coefficients below $R=0.1$. This resulted in a stable pressure inside the combustor.

The results for $O P 1$ are shown in figure 5.4. The left hand plots show the axial velocity (instantaneous snapshot and averaged over $13 \mathrm{~ms}$ ) and the right hand plots the temperature (instantaneous snapshot and averaged). The axes $x$ and $y$ have been normalized with the jet diameter $D_{j e t}$. The white line in the velocity contour plots

${ }^{*}$ The dimensionless frequency $f^{*}$ is defined as:

$$
f^{*}=f \frac{L}{c}
$$

with $L$ the total length of the combustion system, from the inlet of the combustion chamber to the outlet of the exhaust duct. 
represents a contour of $u_{a x}=0 \mathrm{~m} / \mathrm{s}$ and indicates the recirculation zone. As discussed in the previous section, the jets are ignited and stabilized by the pilot swirl flame. This can also be seen in the figure: the burned gas from the swirl flame flows between the recirculation zone and the conical wall to the base of the jet. The flame tip is approximately located at $x / D_{\text {jet }}=5$, which means the flame is 5 jet diameters long.

The flow field for $O P 2$ looks very similar, although the flame is slightly longer.

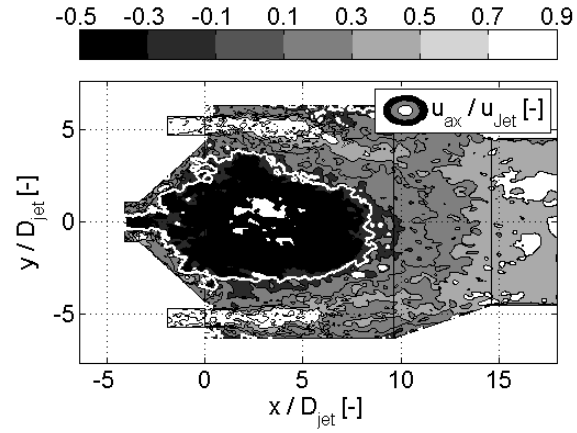

(a) Instantaneous axial velocity, normalized with the mean jet velocity. Thick white line represents contour of $u_{a x}=0$.

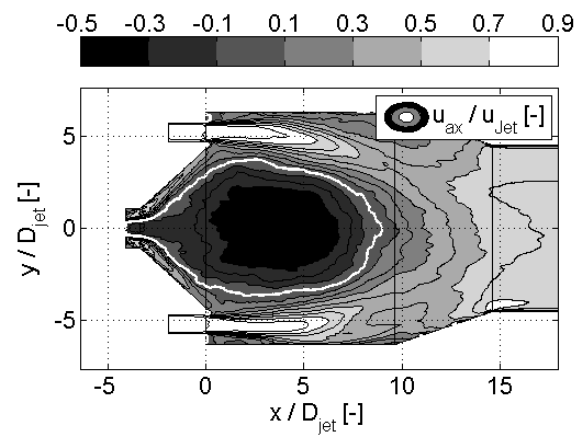

(c) Axial velocity averaged over $13 \mathrm{~ms}$, normalized with the mean jet velocity. Thick white line represents contour of $u_{a x}=0$.

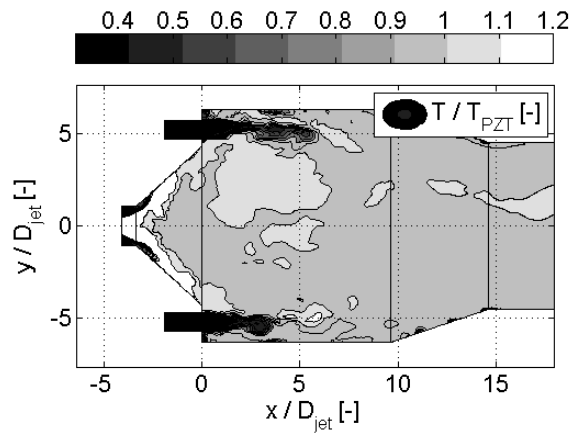

(b) Instantaneous temperature, normalized with the mean adiabatic flame temperature of the combustion chamber $T_{P Z T}$.

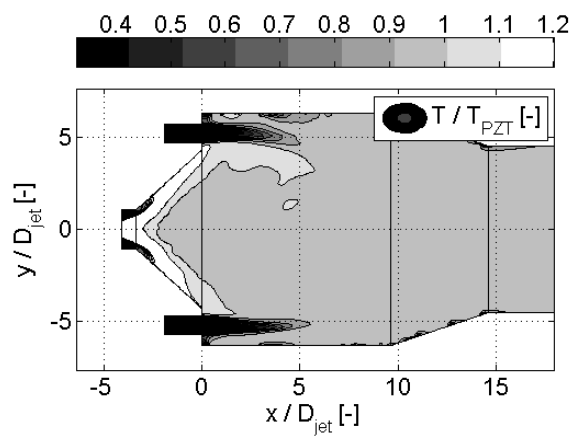

(d) Temperature averaged over $13 \mathrm{~ms}$, normalized with the mean adiabatic flame temperature of the combustion chamber $T_{P Z T}$.

Figure 5.4: Contour plots of the steady flow field for $O P 1$. The axes $x$ and $y$ have been normalized with the jet diameter $D_{j e t}$. The geometry upstream upstream of the combustion chamber including the fuel injectors is not shown here. A sketch is given in figure 5.1. 


\subsection{Results of the simulation with excitation}

\subsubsection{Postprocessing algorithm}

Next the Flame Describing Function is determined. In this chapter it is investigated if the nonlinear flame response of an industrial combustion chamber can be modeled using a MISO flame model, such as the model given by equation 1.10. This equation can be written in the time domain using a $n-\tau$ formulation as:

$$
\begin{aligned}
\frac{Q^{\prime}}{Q_{0}} & =\frac{Q_{a i r}^{\prime}}{Q_{0}}+\frac{Q_{\text {fuel }}^{\prime}}{Q_{0}} \\
& =\underbrace{n_{\text {air }} \frac{u_{C B}^{\prime}\left(t-\tau_{\text {air }}\right)}{u_{0}}}_{\text {Velocity fluctuations }}+\underbrace{n_{f u e l} \frac{\Phi_{\text {Inj }}^{\prime}\left(t-\tau_{\text {fuel }}\right)}{\Phi_{0}}}_{\text {Equivalence ratio fluctuations }}
\end{aligned}
$$

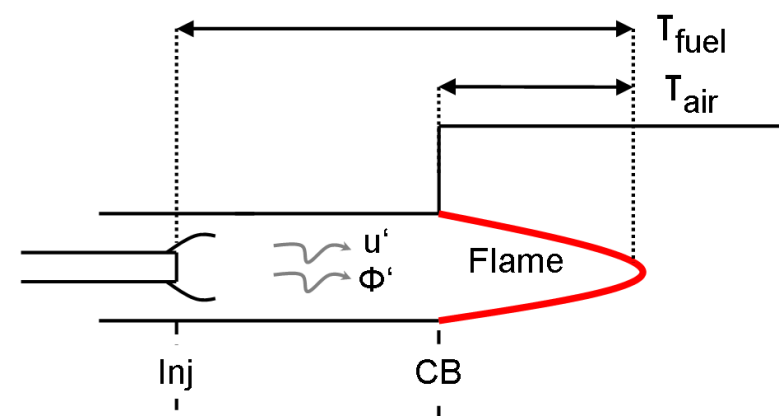

Figure 5.5: Definition of air and fuel time lag.

This model takes into account both heat release fluctuations in response to velocity (mass flow) fluctuations $Q_{a i r}^{\prime}$ and heat release fluctuations in response to fluctuations in the equivalence ratio $Q_{\text {fuel }}^{\prime}$. Here $\tau_{a i r}$ is the time lag between $Q_{a i r}^{\prime}$ and the velocity fluctuations at the inlet of the combustion chamber, indicated with " $\mathrm{CB}^{\prime \prime}$ in figure 5.5. The equivalence ratio fluctuations are determined at the position of fuel injection (indicated with "Inj" in figure 5.5). The parameters $n_{\text {air }}$ and $n_{\text {fuel }}$ are the gains of velocity and equivalence ratio fluctuations respectively. These quantities are dependent on the frequency and, in case of large fluctuations, on the excitation amplitude.

It should be noted that the MISO model does not contain cross terms between equivalence ratio fluctuations and velocity fluctuations; both contributions are linearly superimposed. Therefore the heat release model may introduce some error,

${ }^{*}$ The $n-\tau$ parameters are related to the Flame Describing Function as:

$$
\begin{array}{cc}
n_{\text {air }}=\left|\mathcal{F}_{u}\right| & \tau_{\text {air }}=\frac{\angle \mathcal{F}_{u}}{2 \pi f} \\
n_{\text {fuel }}=\left|\mathcal{F}_{\Phi}\right| & \tau_{\text {fuel }}=\frac{\angle \mathcal{F}_{\Phi}}{2 \pi f}
\end{array}
$$


because it does not take into account these cross terms. During the nonlinear stability analysis, which is discussed in section 5.5, it will be shown however that the model does lead to sensible predictions of the limit cycle pressure amplitude.

The Single Input, Single Output FDF of the swirl stabilized flame, discussed in the previous chapter, was determined using harmonic excitation at the air inlet, both for the perfectly premixed and partially premixed case. For the combustion system studied in this chapter, which is also partially premixed, a MISO Flame Describing Function will be determined however.

When only harmonic excitation at the air inlet only would be applied to a MISO case, while keeping the fuel mass flow constant, a problem would arise. The velocity and equivalence ratio can not be excited independently, which means that both types of fluctuations are always present at the same time. From the time series of the heat release fluctuations, it cannot be directly determined which part of the heat release fluctuation is caused by which type of fluctuation. Equation 5.2 contains 4 unknown parameters $\left(n_{\text {air }}, n_{\text {fuel }}, \tau_{\text {air }}, \tau_{\text {fuel }}\right)$. However, only 2 quantities can be solved independently (one $n$ and one $\tau$ parameter) if harmonic excitation at the air inlet only would be used here. This means that a unique solution does not exist. For this reason, in order to obtain a MISO FDF, both the air and fuel inlet need to be excited.

In order to overcome this problem, the following strategy was developed within this work. For each excitation amplitude not one, but two simulations were used:

- Sim 1: Excitation of the fuel line mass flow. The air mass flow was not excited.

- Sim 2: Excitation of the air mass flow. The fuel line mass flow was not excited.

This strategy results in two independent time series of the heat release fluctuations. A unique solution for $n_{\text {air }}, n_{\text {fuel }}, \tau_{\text {air }}$ and $\tau_{\text {fuel }}$ can now be determined if additionally the following important assumption is made:

The values for $n_{\text {air }}, n_{\text {fuel }}, \tau_{\text {air }}, \tau_{\text {fuel }}$ are identical in Sim 1 and $\operatorname{Sim} 2$.

This assumption is valid for cases where the contributions of $Q_{a i r}^{\prime}$ and $Q_{f u e l}^{\prime}$ can be linearly superimposed and no cross terms exist. As mentioned, this may not be strictly true in the nonlinear regime, but during the nonlinear stability analysis it will be shown however that nonetheless sensible predictions of the limit cycle pressure amplitude are obtained.

Using this assumption, the $n-\tau$ values have been iteratively determined, as indicated in the flow chart in figure 5.6. First an initial value for $n_{\text {air }}$ and $\tau_{\text {air }}$ was estimated.* These initial values were used as an input to the postprocessing script of $\operatorname{Sim} 1$. With these initial values, the total heat release fluctuation could be split into heat release fluctuations caused by velocity fluctuations $\left(Q_{a i r}^{\prime}\right)$ and heat release fluctuations caused by equivalence ratio fluctuations $\left(Q_{f u e l}^{\prime}\right)$. By taking the Fourier transformation of $Q_{f u e l}^{\prime}$, the parameters $n_{f u e l}$ and $\tau_{f u e l}$ could be determined.

\footnotetext{
*The initial values were $n_{\text {air }}=1$ and $\tau_{\text {air }}=L_{\text {flame }} / u_{\text {jet }} \approx 5 D_{\text {jet }} / u_{j e t}$. However, the $n-\tau$ values always converged to the same values if different initial values were used (e.g. $\tau=0 \mathrm{~s}$ ).
} 
Next, these newly found values for $n_{\text {fuel }}$ and $\tau_{f u e l}$ are used as an input in a second postprocessing script for $\operatorname{Sim} 2$. Likewise, the total heat release fluctuation is first split into fluctuations of the individual components. This time however, values for $n_{\text {air }}$ and $\tau_{\text {air }}$ are determined by Fourier transforming $Q_{a i r}^{\prime}$.

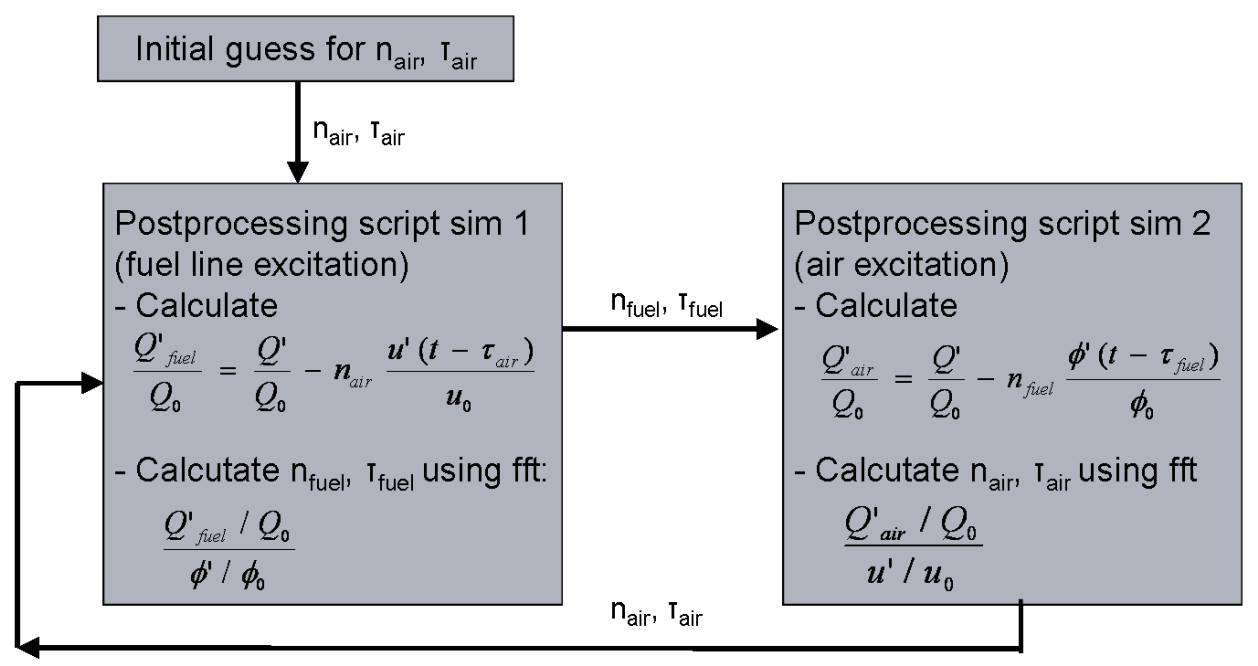

Figure 5.6: Postprocessing algorithm for the MISO Flame Describing Function.

The values for $n_{\text {air }}$ and $\tau_{a i r}$ are then back-substituted in the postprocessing script for $\operatorname{Sim} 1$, which is the start for the second iteration. The whole procedure is repeated until all four parameters $\left(n_{\text {air }}, n_{\text {fuel }}, \tau_{\text {air }}, \tau_{\text {fuel }}\right)$ have converged to a constant value. About 5 iteration loops were required in order to reach the convergence criterion that for each parameter, the ratio between the value of the current and the previous iteration should be smaller than $\epsilon=10^{-4}$.

\subsubsection{Flame Describing Function}

In figure 5.7 the MISO Flame Describing function at a dimensionless frequency of $f^{*}=1.05$ is shown. Note that the $n-\tau$ values obtained from the postprocessing algorithm have been converted into $|\mathcal{F}|$ and $\angle F$ again, so that the values are dimensionless and in the same form as the Flame Describing Functions presented in Chapter 4. It should also be noted that the results are not shown as function of frequency (this would not make sense since only 1 frequency has been simulated), but only as a function of the excitation amplitude.

The top plots show the magnitude of the FDF, the bottom plots show the phase. The results for $O P 1$ are shown in the left column, the results for $O P 2$ to the right. For $O P 1$ simulations at three different excitation amplitudes have been performed (see table 5.1, for $O P 2$ only at $u^{\prime} / u_{0}=0.1$. The main conclusions that can be drawn from these figures are: 


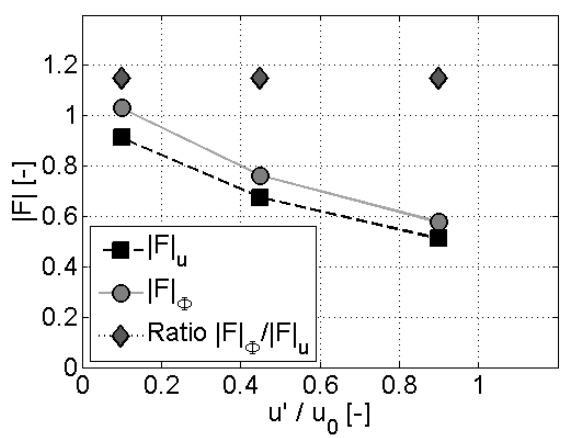

(a) OP1 Magnitude.

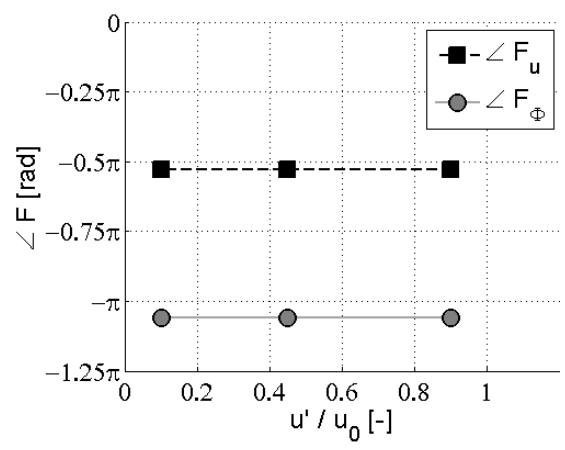

(c) OP1 Phase.

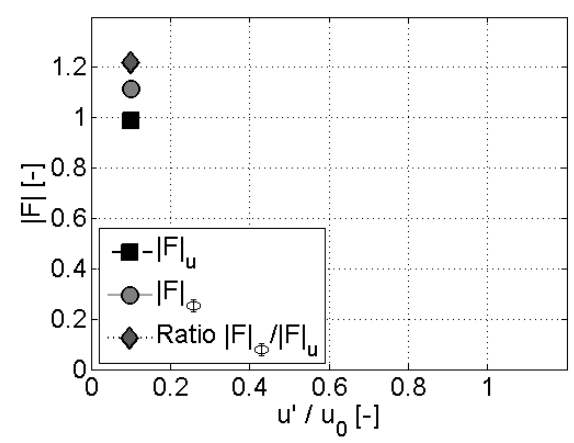

(b) OP2 Magnitude.

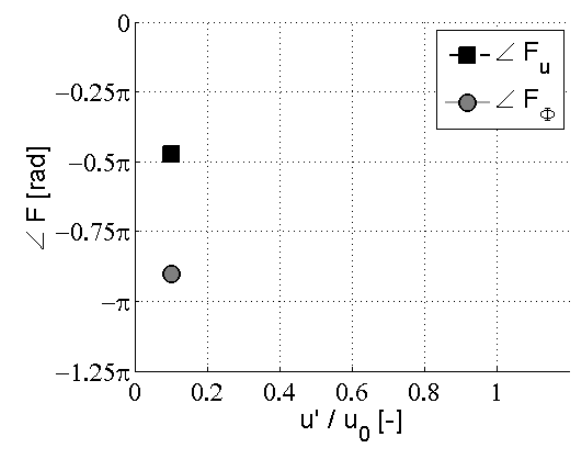

(d) OP2 Phase.

Figure 5.7: Flame Describing Function - as function of excitation amplitude.

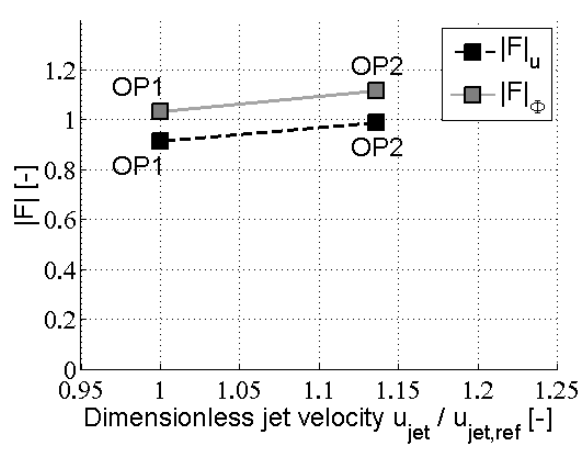

(a) Magnitude $\left(u^{\prime} / u_{0}=0.1\right)$.

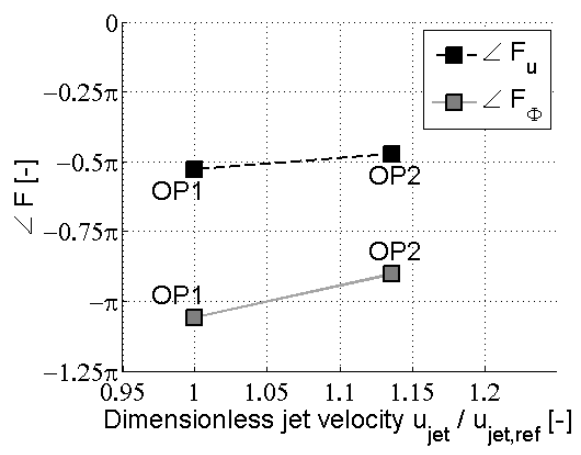

(b) Phase $\left(u^{\prime} / u_{0}=0.1\right)$.

Figure 5.8: Comparison of the Flame Describing Function of $O P 1$ and $O P 2$. 
- Both the magnitude of $|\mathcal{F}|_{u}$ and $|\mathcal{F}|_{\Phi}$ decreases with increasing excitation amplitude for $O P 1$, indicating saturation of the heat release. The gain $|\mathcal{F}|_{u}$ is slightly smaller than the gain $|\mathcal{F}|_{\Phi}$. The ratio between both gains stays approximately constant and seems to be independent of the excitation amplitude.

- The phases $\angle \mathcal{F}_{u}$ and $\angle \mathcal{F}_{\Phi}$ do not significantly depend on excitation amplitude. The phase $\angle \mathcal{F}_{\Phi}$ is significantly larger than $\angle \mathcal{F}_{u}$ (more negative), which can be explained by the longer time delay $\tau_{f u e l}$ of equivalence ratio fluctuations (see figure 5.5).

- The magnitude of the FDF for $O P 2\left(u^{\prime} / u_{0}=0.1\right)$ is slightly larger than the magnitude of the FDF for $O P 1$. The phase of $O P 2$ is slightly smaller (less negative) than the magnitude of $O P 1$. Equivalence ratio fluctuations are convectively transported in the premixing passage. A larger jet velocity thus results in a smaller time lag / phase.

To illustrate the last of these conclusions, the magnitude and phase are also shown as a function of jet velocity in figure 5.8 .

It should be noted that all data points shown in figure 5.7 and 5.8 are consistent, without outliers. The trends are as expected. This gives confidence in the postprocessing algorithm that was introduced in figure 5.6.

\subsection{Nonlinear stability analysis}

The MISO Flame Describing Function, predicted in the previous section, is now used to predict the limit cycle pressure amplitude with the Generalized Instability Model. First the model setup will be discussed, followed by the results of the nonlinear stability analysis.

\subsubsection{Model setup}

As mentioned, the CFD simulations were performed on a reduced domain which ends upstream of the Vane Simulation Section (see figure 5.2). Non-reflecting boundary conditions were used, to prevent unphysical self-excited modes. In the nonlinear stability analysis however, the modeled geometry and boundary conditions should match the actual domain and boundary conditions of the test rig.

The modeled geometry can be seen in figure 5.9(a). The figure shows the effective diameter $\left(D_{\text {eff }}=\sqrt{4 A / \pi}\right)$ of the different domains over the axial coordinate $x / L$. The model starts upstream of the jets, at the location where the air from the plenum enters the burner. Due to the high area contraction ratio from plenum to burner, the pressure perturbations at this location are assumed to be zero. Therefore, a pressure node and a velocity anti node at this location are a suitable acoustic inlet condition for the thermo-acoustic model.

The model ends downstream of the exhaust section. At this location (see figure 5.2), the acoustic domain is terminated with a choked nozzle, so that the outlet 


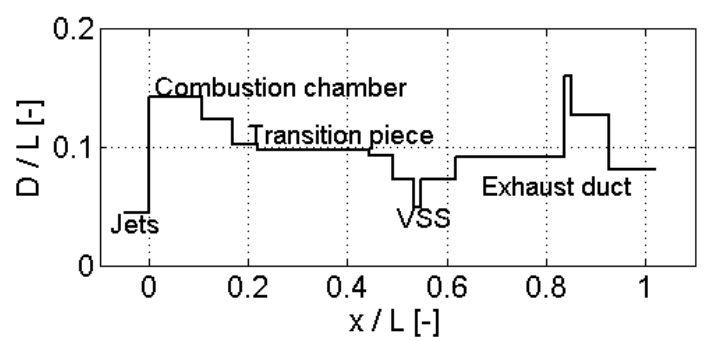

(a) Modeled geometry, including exhaust section.

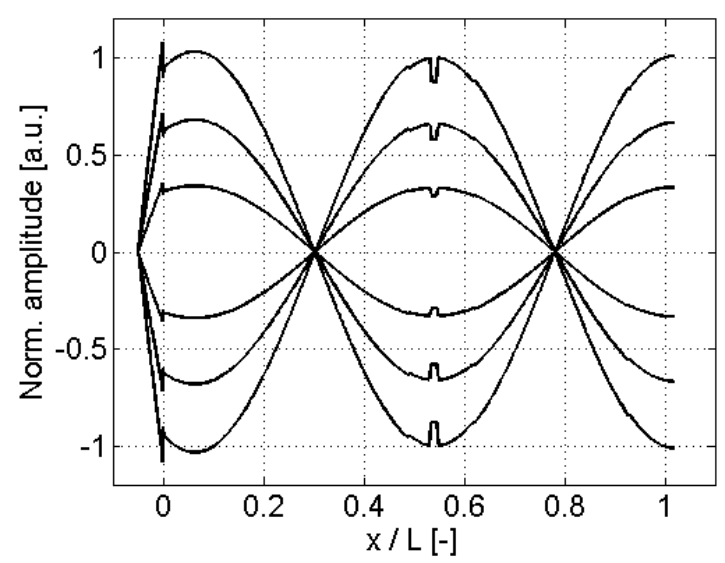

(b) Pressure mode shape.

Figure 5.9: Domain for the nonlinear stability analysis and pressure mode shape. 
boundary condition is well defined as well. The pressure mode shape of the unstable $1 \mathrm{~L}$ mode is shown in figure $5.9(\mathrm{~b})$. The mode has a pressure node at the inlet $(x / L \approx-0.05)$, an anti node at the inlet to the combustion chamber $(x / L=0)$ and a second anti node at the outlet $(x / L=1)$.

\section{Losses}

In order to predict the eigen frequencies and the linear stability behavior correctly, it would be sufficient to specify $R=-1$ at the inlet $\left(p^{\prime}=0\right)$ and $R=1$ at the outlet $\left(u^{\prime}=0\right)$. However, in order to predict the limit cycle pressure amplitude correctly, the losses at these locations are required. Unfortunately, the exact reflection coefficients are not known from measurements or simulations and are difficult to determine. Therefore, the outlet reflection coefficient $R_{\text {out }}$ was estimated using an analytical expression from Dowling for choked outlets [24]:

$$
R_{\text {out }}=\frac{2-M a(\gamma-1)}{2+M a(\gamma-1)}
$$

Here $M a$ is the Mach number in the exhaust section, immediately upstream of the choked nozzle. Applying this expression leads to a value of $R_{\text {out }}=0.95$ for $O P 1$ (which means about $5 \%$ losses). The uncertainty on this reflection coefficient is assumed to be in the order of $\Delta R_{\text {out }}= \pm 0.025$, corresponding to $\pm 2.5 \%$ losses.

At the inlet, which was not choked, an analytical expression to estimate $R_{i n}$ was not available. For this configuration, the assumption has been made that the lower bound reflection coefficient is $R=-1$ (no losses) and the upper bound $R=-0.95$ ( $5 \%$ losses). The baseline value is assumed to be $R_{i n}=-0.975$ and the uncertainty $\Delta R_{\text {in }}= \pm 0.025$.

The fact that in industrial combustion systems, reflection coefficients are harder to determine than reflection coefficients of generic test rigs, is a general difficulty in the practical application of thermo-acoustic models to industrial combustion systems.

\section{Heat release model}

In order to use the MISO Flame Transfer Function which was determined from the CFD simulation with GIM, equivalence ratio fluctuations should first be related to acoustic velocity and/or pressure fluctuations.

Dowling et al. $[4,26]$ derived that the equivalence ratio fluctuations can be related to the velocity fluctuations at the location of fuel injection as:*

$$
\left(\frac{\Phi^{\prime}}{\Phi_{0}}\right)_{\mathrm{inj}}=-\left(\frac{m^{\prime}}{m_{0}}\right)_{\mathrm{inj}}=-\left(\frac{u^{\prime}}{u_{0}}\right)_{\mathrm{inj}}
$$

The final heat release model implemented in GIM is obtained by inserting equation 5.4 into equation 1.10 and writing the result in the time domain:

\footnotetext{
*Equation 5.4 is exact for constant fuel mass flow. Due to the large Mach number in the fuel nozzles $(M a \approx 0.8)$, the fuel mass flow is almost choked and not expected to fluctuate significantly.
} 


$$
\begin{aligned}
\frac{Q^{\prime}}{Q_{0}}= & \underbrace{\left|\mathcal{F}_{u}\right|\left(\left|u_{\mathrm{CB}}^{\prime}\right|\right)\left(\frac{u^{\prime}\left(t-\angle \mathcal{F}_{u} / 2 \pi f\right)}{u_{0}}\right)_{\mathrm{CB}}}_{\text {Velocity fluctuations }} \\
& \underbrace{-\left|\mathcal{F}_{\Phi}\right|\left(\left|u_{\text {Inj }}^{\prime}\right|\right)\left(\frac{u^{\prime}\left(t-\angle \mathcal{F}_{\Phi} / 2 \pi f\right)}{u_{0}}\right)_{\text {Inj }}}_{\text {Equivalence ratio fluctuations }}
\end{aligned}
$$

Here the subscripts "Inj" and "CB" stand for evaluated at the location of fuel injection and evaluated at the inlet of the combustion chamber respectively. Their locations are indicated in figure 5.5 .

\subsubsection{Results}

\section{Operating point $O P 1$}

The baseline results of the nonlinear stability analysis for $O P 1$ (with $R_{\text {inlet }}=-1$ and $R_{\text {out }}=0.95$ ) are presented in figure 5.10. First the acoustic pressure and acoustic velocity are shown as a function of time in subfigures 5.10(a) and 5.10(b). After about 0.6 seconds a limit cycle is reached, with pressure amplitude $p^{\prime} / p_{0}=0.083$ and velocity amplitude $u^{\prime} / u_{0}=1.2$. During a part of the acoustic cycle $u^{\prime} / u_{0}>1$, which indicates that mean flow reversal may occur.

A spectrum of the results is shown in subfigure 5.10(c). The root mean square pressure predicted by GIM was $p_{r m s} / p_{0}=0.061$, whereas a rms-pressure of $p_{r m s} / p_{0}=$ 0.035 was measured for $O P 1$. This means that the pressure amplitude was over predicted by $74 \%$ ( $+4.8 \mathrm{~dB}$ on a SPL scale). The dimensionless frequency $f^{*}$ in the simulation was $6 \%$ smaller than the measured unstable frequency.

As mentioned in the previous section, the reflection coefficients at the inlet and outlet of the rig were not well known. For both reflection coefficients, the uncertainty was estimated. In figure 5.11 the limit cycle pressure amplitude can be seen as a function of the inlet (a) and outlet (b) reflection coefficient. During the sensitivity study for the inlet reflection coefficient, the outlet reflection coefficient was kept constant and vice versa. With the estimated uncertainty in the reflection coefficient of $\Delta R= \pm 0.025$, an uncertainty in the limit cycle pressure amplitude of almost a factor 2 is found. This means that this uncertainty in reflection coefficient is likely a root cause for the over prediction of the limit cycle pressure amplitude in the simulation.

It should be noted however that the accuracy of the limit cycle and frequency prediction is in the same order of magnitude as the results found in the previous chapters (Chapter 2 and 4). Therefore the baseline result is considered an acceptable result.

\section{Operating point $O P 2$}

As mentioned before, the only difference between $O P 1$ and $O P 2$ is the larger jet velocity (see figure 5.3). All other conditions were identical. The larger jet velocity 


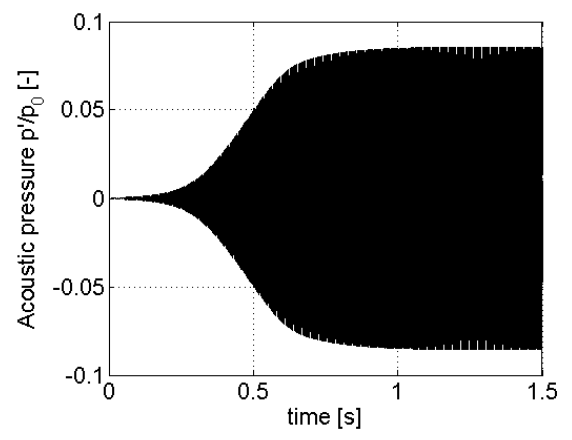

(a) Acoustic pressure.

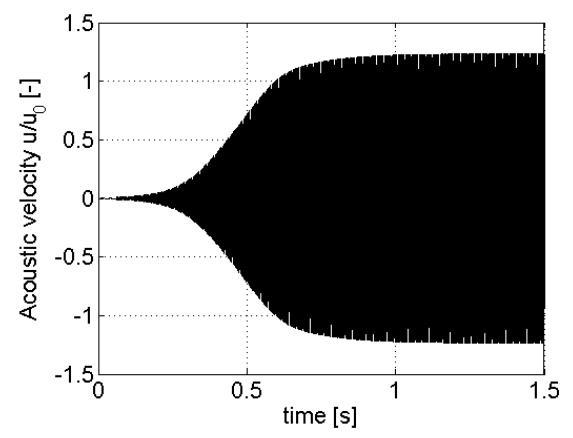

(b) Acoustic velocity.

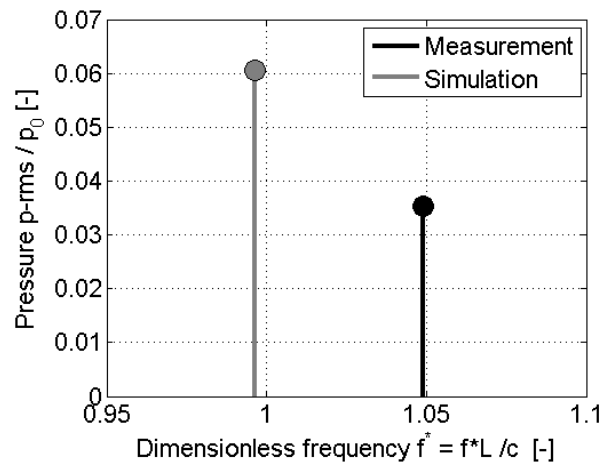

(c) Spectrum.

Figure 5.10: Baseline results of the nonlinear stability analysis for $O P 1$.

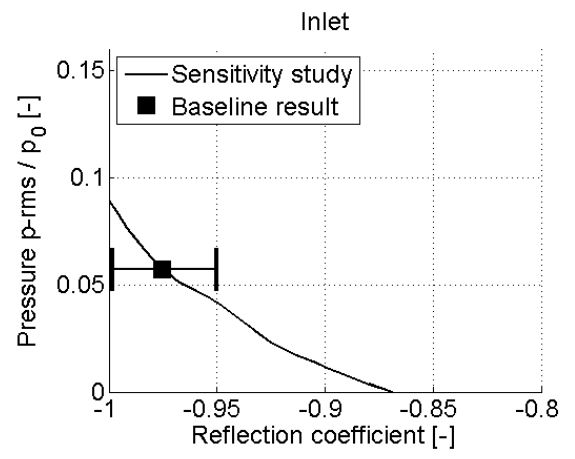

(a) Influence of the inlet reflection coefficient. The outlet reflection coefficient was kept constant in this analysis $\left(R_{\text {out }}=0.95\right)$.

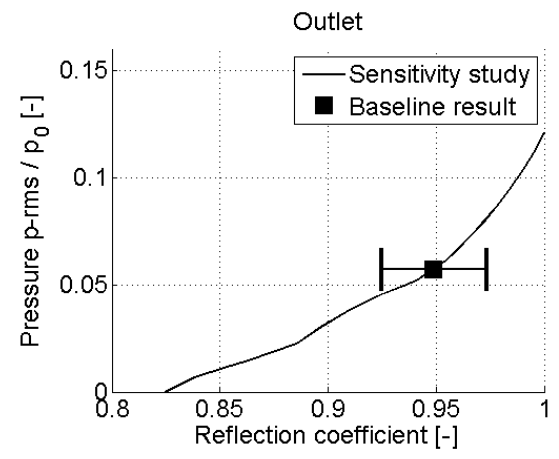

(b) Influence of the outlet reflection coefficient. The inlet reflection coefficient was kept constant in this analysis $\left(R_{i n}=-0.975\right)$.

Figure 5.11: Influence of the reflection coefficient on the predicted limit cycle pressure amplitude for $O P 1$. The markers indicate the baseline results of figure 5.10 and the error bars show the estimated uncertainty of the reflection coefficient. 
resulted in both a larger gain and a larger phase (smaller time lag) of the Flame Describing Function. The comparison of the FDF for $O P 1$ and $O P 2$ was shown before in figure 5.8 .

The Generalized Instability Model predicts a neutrally stable mode with a growth rate $\Gamma=-1 \mathrm{rad} / \mathrm{s}$ for $O P 2$. The fact that $O P 2$ is neutrally stable and $O P 1$ unstable can be explained with the larger phase of the FDF for $O P 2$. A neutrally stable mode is in agreement with figure 5.3. As can be seen in figure 5.3, operating point $O P 2$ was stable, but lies very close to the stability boundary. Only a very small reduction in jet velocity is already expected to significantly increase the limit cycle pressure amplitude.

This is an important result, because it shows that GIM predicts the trends correctly.

\subsubsection{Analysis of the MISO Model}

Both the results for $O P 1$ and $O P 2$ are in reasonable agreement with experimental data. However, the advantage of the MISO model over a SISO model has not been shown yet. In this section it is investigated what the individual roles of equivalence ratio and velocity fluctuations are.

Equation 5.5 consists of an equivalence ratio fluctuations and a velocity fluctuations contribution. In GIM, it is possible to separately activate or deactivate individual source terms. First the linear growth rate obtained from these individual source terms is investigated in table 5.2:

\begin{tabular}{ll}
\hline Activated mechanisms & Growth rate \\
\hline Only damping $\left(Q^{\prime}=0\right)$ & $\Gamma=-7.9 \mathrm{rad} / \mathrm{s}$ \\
Damping and full HR model $\left(Q^{\prime}=Q_{f u e l}^{\prime}+Q_{\text {air }}^{\prime}\right)$ & $\Gamma=+13.1 \mathrm{rad} / \mathrm{s}$ \\
Damping and eq. ratio fluctuations $\left(Q^{\prime}=Q_{\text {fuel }}\right)$ & $\Gamma=-8.9 \mathrm{rad} / \mathrm{s}$ \\
Damping and velocity fluctuations $\left(Q^{\prime}=Q_{\text {air }}^{\prime}\right)$ & $\Gamma=+14.2 \mathrm{rad} / \mathrm{s}$ \\
\hline
\end{tabular}

Table 5.2: Contribution of individual source terms to the linear growth rate for $O P 1$.

First it can be seen that the acoustics of the system damp out when the complete heat release (HR) model is deactivated (Only damping). When both damping and the full HR model is activated, the combustor becomes unstable $(\Gamma>0)$, as expected. A very interesting result is found when only a part of the HR model is used. When only the source term due to equivalence ratio fluctuations is used $\left(Q^{\prime}=Q_{\text {fuel }}^{\prime}\right)$, the combustor becomes actually more stable than without a HR model. On the other hand, when only the velocity fluctuations source term is activated, a larger growth rate is found than the growth rate obtained with the full HR model.

From this it can be concluded that velocity fluctuations excite the thermo-acoustic oscillations and equivalence ratio fluctuations slightly damp them. This conclusion is also in agreement with results from Gövert, who performed a self-excited CFD simulation of the same configuration [34]. Gövert also identified velocity fluctuations as the driving mechanism leading to thermo-acoustic instability. 
This result is very valuable. First it explains what the main instability mechanism is for this partially premixed flame. Secondly it helps giving directions for improvement of the design of the burner with respect to thermo-acoustic instability. The influence of equivalence ratio fluctuations can be easily changed independently of the velocity fluctuations: namely by changing the axial position of fuel injection.

First the axial transport of equivalence ratio fluctuations is investigated in figure 5.12. The equivalence ratio fluctuations on two sampling planes are shown, as a function of the dimensionless time $t^{*}$, which is defined as $t^{*}=2 \pi \mathrm{ft}$. The sampling plane $X_{P}$ is located slightly downstream of the fuel injectors and $X_{C B}$ at the inlet of the combustion chamber. Clearly a time difference can be seen between the equivalence ratio at $X_{P}$ (solid black curve) and $X_{C B}$ (solid gray curve). The dashed black curve shows the same data as the solid black curve, but has been translated to the right by $\Delta t^{*}=2 \pi f \Delta t_{\text {conv }}=2 \pi f\left(X_{C B}-X_{P}\right) / u_{j e t}$. Since the dashed black curve and gray curve approximately coincide, it can be concluded that equivalence ratio fluctuations are approximately transported with the convective velocity.

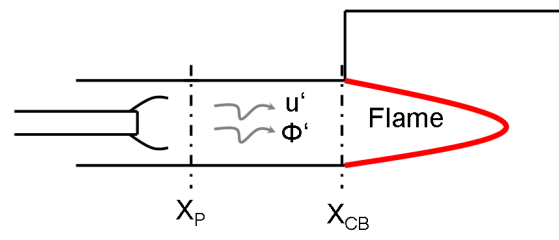

(a) Position of sampling planes $X_{P}$ and $X_{C B}$.

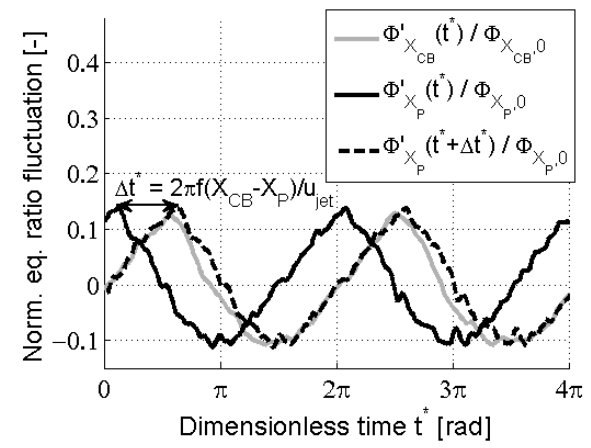

(b) Equivalence ratio fluctuations on sampling planes $X_{P}$ and $X_{C B}$. The time has been normalized by the frequency.

Figure 5.12: Propagation of equivalence ratio fluctuations in the premixing passage, between sampling planes $X_{P}$ and $X_{C B}$.

This insight can now be used to estimate the effect on $\angle F_{\Phi}$ when the axial position of fuel injection $X_{i n j}$ is changed. A change in axial position of $\Delta x_{i n j}$ results in a change of $\angle F_{\Phi}$ of approximately:

$$
\Delta\left(\angle F_{\Phi}\right)=-2 \pi f \Delta \tau_{f u e l}=-2 \pi f \frac{\Delta x_{i n j}}{u_{j e t}}
$$

Applying equation 5.6, the acoustic pressure amplitude can now be predicted as function of the location of the fuel injectors. It can be seen in figure 5.13 that when the fuel injectors are moved away from the combustion chamber (in negative $x$-direction), larger pressure amplitudes are predicted. On the other hand when the injectors are moved downstream (in positive x-direction), the burner becomes more stable (smaller pressure amplitudes). So with regard to thermo-acoustic stability, it 
is beneficial to have a short premixing length ${ }^{\dagger}$.

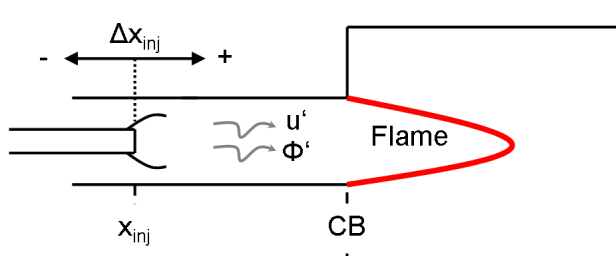

(a) Axial position of fuel injector.

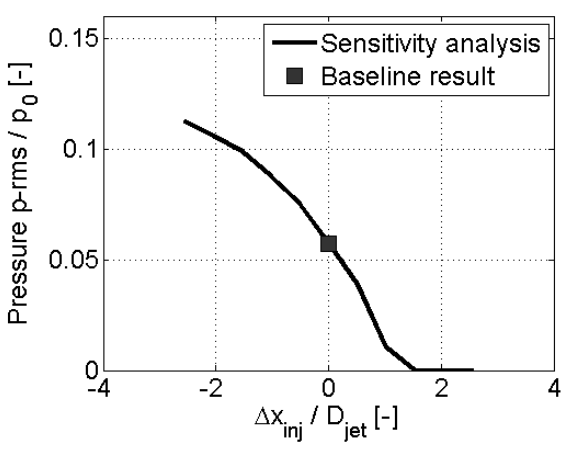

(b) Acoustic pressure amplitude as function of position of axial fuel injector. $\Delta x_{i n j}$ has been normalized by the jet diameter $D_{j e t}$.

Figure 5.13: Acoustic pressure amplitude as function of axial position of fuel injector.

These GIM results are in agreement with experimental findings from high pressure tests [8]. The experimental data for which the fuel injection position was varied were taken at slightly different operating conditions than the operating conditions investigated with CFD and are therefore not shown together with the numerical data in figure 5.13 .

In the test campaign [8], a measurement was performed with the fuel injectors located at $\left(\Delta x_{i n j} / D_{j e t}=0\right)$ and at $\left(\Delta x_{i n j} / D_{j e t}=-1.5\right)$. For $\Delta x_{i n j} / D_{j e t}=0$ a pressure amplitude of $p_{r m s} / p_{0}=0.0057$ was measured, which increased to $p_{r m s} / p_{0}=$ 0.0170 when the fuel injectors were moved upstream (which is about a factor 3) [8]. The same trends may be expected for the operating point which has been investigated in this chapter.

A SISO model may have been able to predict the same limit cycle pressure amplitude, but would not have been able to shed some light on the individual role of equivalence ratio and velocity fluctuations. Therefore it would not have been possible to identify the position of the fuel injectors as an important design parameter. This demonstrates the additional benefit of using a MISO model.

\subsection{Conclusions}

In this chapter it was shown that also the limit cycle pressure amplitude of an industrial combustion system, the DOC burner, can be successfully predicted. Particular focus was on the use of a Multiple Input, Single Output heat release model, which takes into account both equivalence ratio fluctuations and velocity fluctuations. The

\footnotetext{
$\dagger$ The effect on emissions (e.g. NOx) on the other hand is most likely negative when the fuel injector is moved towards the flame, because this leads to an increased unmixedness of fuel and air.
} 
FDF consisted of two parts: $\mathcal{F}_{u}$ (velocity fluctuations) and $\mathcal{F}_{\Phi}$ (equivalence ratio fluctuations).

The MISO Flame Describing Function was predicted for two operating points. The main difference between those operating points was the jet velocity in the premixing passage upstream of the flame (which was $14 \%$ larger for the second operating point). It was found that both the phase $\angle \mathcal{F}_{u}$ and the phase $\angle \mathcal{F}_{\Phi}$ were smaller for the operating point with the largest jet velocity. The magnitudes $\left|\mathcal{F}_{u}\right|$ and $\left|\mathcal{F}_{\Phi}\right|$ were not signifantly different. However the magnitude depended on the excitation amplitude, which means that the heat release saturated.

Next the limit cycle pressure amplitude was predicted using GIM. It was found that the operation point with the largest jet velocity was neutrally stable. This agrees with experimental data. The operating point with the smallest jet velocity appeared to be unstable in both experiments and simulations. The frequency in the simulation was predicted within $6 \%$ (compared to the measurements). The amplitude from the simulations was $74 \%$ larger however.

A difficulty in setting up the GIM model was the fact that the reflection coefficient at the inlet and outlet were not well known. Therefore these were estimated using analytical correlations. A sensitivity analysis on the boundary conditions led to the conclusion that a $5 \%$ uncertainty in reflection coefficient may double the predicted limit cycle pressure amplitude. This uncertainty may be the reason for the $74 \%$ difference between predicted and measured limit cycle pressure amplitude that has been observed. However, the accuracy of the limit cycle prediction was in the same order of magnitude as the results found in the previous chapters (Chapter 2 and 4) and is therefore considered an acceptable result.

Finally the individual contribution of equivalence ratio fluctuations and velocity fluctuations was analyzed in more detail. It was found that velocity fluctuations amplify the thermo-acoustic instabilities, whereas equivalence ratio fluctuations damp them. This result was then used to propose a design change that improves the thermo-acoustic stability behavior of the burner. It was found that the stability of the burner could be improved by reducing the phase $\angle \mathcal{F}_{\Phi}$. This could be done by shifting the location of the fuel injection downstream, towards the flame.

It would not have been possible to identify the position of the fuel injectors as an important design parameter using a SISO model. This analysis therefore demonstrates the additional benefit of MISO models. 


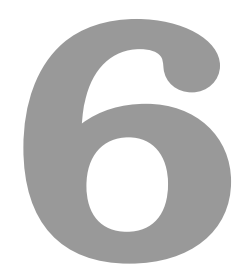

\section{Conclusions and recommendations}

In this chapter the main conclusions from this work are summarized. The conclusions are presented based on the research questions that were posed in Chapter 1 of this dissertation.

The conclusions are followed by recommendations for follow up research.

\subsection{Answering the research questions}

1. Can the limit cycle pressure amplitude be predicted using a one-dimensional stability model?

Yes. A thermo-acoustic stability model, the Generalized Instability Model, was extended with nonlinear heat release models, in the form of the Flame Describing Function. The limit cycle pressure amplitude was successfully predicted for different test cases. A generic bluff body stabilized flame (Chapter 2), a generic swirl flame (Chapter 4 ) and an industrial combustion system (Chapter 5) were studied.

\begin{tabular}{llll}
\hline Test Case & $\begin{array}{l}\text { Frequency } \\
\left(\mathbf{f}_{\text {sim }} / \mathbf{f}_{\text {meas }}\right)\end{array}$ & $\begin{array}{l}\text { Pressure amplitude } \\
\left(\mathbf{p}_{\text {sim }}^{\prime} / \mathbf{p}_{\text {meas }}^{\prime}\right)\end{array}$ & Reference \\
\hline Bluff body flame & $+8 \%$ & $+12 \% /+1.0 \mathrm{~dB}$ & Table 2.4 \\
Swirl flame (part. premixed) & $+5 \%$ & $+46 \% /+3.3 \mathrm{~dB}$ & Table 4.7 \\
Industrial combustor (DOC) & $-6 \%$ & $+74 \% /+4.8 \mathrm{~dB}$ & Sec. 5.5.2 \\
\hline
\end{tabular}

Table 6.1: Comparison of frequency and dynamic pressure amplitude between measurements (meas) and simulations (sim) for the baseline results of the different test cases studied. 
In order to give an idea about the accuracy of the tool, the baseline results presented in the different chapters are summarized and compared to experimental data in table 6.1. It can be seen that the frequency of the instability is typically predicted within $5 \%$ to $10 \%$, whereas up to $74 \%$ deviation in the pressure amplitude between measurents and experiments was found (on a linear scale). However, the pressure amplitude was always predicted within the right order of magnitude. On a sound pressure level scale, the pressure amplitude could be predicted within $5 \mathrm{~dB}$.

An important conclusion is that the accuracy in the prediction of the limit cycle pressure amplitude is very sensitive to both the Flame Describing Function (FDF) and the acoustic boundary conditions. Parametric analysis on the phase of the FDF showed that a phase change of less than $10 \%$ can double the limit cycle pressure amplitude (see figure 2.6 or figure 4.20). Parametric analysis on the boundary conditions showed that an uncertainty of $\Delta R= \pm 0.025$ in the reflection coefficient can result in uncertainties of the limit cycle pressure amplitude of up to $100 \%$ as well (see figure 2.7 or figure 5.11).

In conclusion, prediction of the limit cycle pressure amplitude using a 1D stability model is well possible. However, it can also be concluded that the results from a nonlinear limit cycle calculation are only relevant when the sensitivity on the boundary conditions and Flame Describing Function is taken into account, since changes in the pressure amplitude may be large for small changes in those parameters. Given this high sensitivity, an improvement of the accuracies in table 6.1 would be hard to achieve. An advantage of the 1D acoustic code, however, is that quick parameter sweeps are possible, so designs can quickly be screened with respect to thermo-acoustic instability.

\section{Can the Flame Describing Function be predicted using CFD (LES)?}

Yes. The Flame Describing Function was predicted both for the swirl stabilized burner from Chapter 4 and for the industrial combustion system from Chapter 5 . The FDF was obtained by perturbing the inlet mass flow using mono-frequency excitation, with different excitation amplitudes. Only for the swirl stabilized flame experimental data on the Flame Describing Function was available.

Both a perfectly premixed and a partially premixed operating point were studied for the swirl stabilized flame. The saturation of the heat release of the flame at a frequency of $115 \mathrm{~Hz}$, which was observed during measurements, was correctly captured in the simulation. At higher frequencies (above $200 \mathrm{~Hz}$ ), no noticeable saturation was found in either the experiments or simulations.

The FDF of the perfectly premixed case was most suited for direct comparison against experimental data, because the heat release fluctuations $Q$ are approximately linearly proportional to $O H^{*}$ fluctuations when no equivalence ratio fluctuations occur. The magnitude of the FDF for the perfectly premixed case agreed well to experimental data. The magnitude of the FDF for the partially premixed case could only be qualitatively compared against experimental data, most likely because of this nonlinearity between $Q$ and $O H^{*}$ fluctuations.

The phase of the FDF was predicted within $0.2 \pi$ for the partially premixed case (corresponding to $10 \%$ of a full acoustic period) and within $0.4 \pi$ for the perfectly 
premixed case ( $20 \%$ of a full acoustic period). The answer to research question 1 already states very clearly what the effects of such differences are. Especially for the perfectly premixed case, it would be recommended to review the CFD setup and combustion model, in order to find out if an improvement of the phase would be possible.

In conclusion, the Flame Describing Function could be successfully predicted using LES. Promising results were found. It would be wise, however, to investigate whether the accuracy of the approach can be be improved.

\section{What is the mechanism leading to saturation of the flame?}

The results revealed that the heat release saturates due to a nonlinear increase of the flame surface with the amplitude of the velocity fluctuations. The saturation of the heat release was investigated in more detail for the the perfectly premixed case of the swirl stabilized burner from Chapter $4 .^{*}$ From an investigation of the different components of the flame surface (resolved, unresolved and total flame surface), it was concluded that the major part of the saturation can be attributed to saturation of the Flame Surface Density $|\nabla c|$.

It was also shown that for the present swirl flame, the flame surface was linearly dependent on the tangential component of velocity fluctuations, but nonlinearly dependent on the axial component. The transfer function of the swirler appeared to be a nonlinear function of excitation amplitude as well. Since in the present study axial and tangential fluctuations were not independently varied, it can not be determined which of the velocity components contributes most to the saturation of the flame surface and heat release and what exactly the impact of the swirler on saturation is. However, the results suggested that an interesting next step would be to design a (numerical) experiment in which both velocity components can be varied independently, to verify their individual impact on the saturation mechanism.

\section{Can the proposed methodology (combination of identification of the Flame Describing Function using LES and a one-dimensional stability tool) be applied to an industrial combustion system to predict limit cycles?}

Yes. In Chapter 5, the limit cycle pressure amplitude of an industrial combustion system, the DOC burner, was successfully predicted. Particular focus was on the use of a Multiple Input, Single Output heat release model, which takes into account both equivalence ratio fluctuations and velocity fluctuations. The FDF consisted of two parts: $\mathcal{F}_{u}$ (velocity fluctuations) and $\mathcal{F}_{\Phi}$ (equivalence ratio fluctuations).

The MISO Flame Describing Function was predicted for two operating points. The main difference between those operating points was the jet velocity in the premixing passage upstream of the flame (which was $14 \%$ larger for the second operating point). It was found that both the phase $\angle \mathcal{F}_{u}$ and the phase $\angle \mathcal{F}_{\Phi}$ were smaller for the operating point with the largest jet velocity. The magnitudes $\left|\mathcal{F}_{u}\right|$ and $\left|\mathcal{F}_{\Phi}\right|$

\footnotetext{
${ }^{*}$ The investigation of the saturation mechanism of the industrial combustion system, presented in Chapter 5 , was beyond the scope of this work.
} 
were not signifantly different. The magnitude of the FDF depended on the excitation amplitude, which means that the heat release saturated.

A difficulty in setting up the GIM model was the fact that the reflection coefficient at the inlet and outlet were not well known. Therefore these were estimated using analytical correlations. As discussed before (Research question 1, this led to some uncertainty in the predicted limit cycle pressure amplitude. The fact that in industrial combustion systems, reflection coefficients are harder to determine than reflection coefficients of generic test rigs may be a general difficulty for industrial combustion systems.

Finally the individual contribution of equivalence ratio fluctuations and velocity fluctuations was analyzed in more detail. It was found that velocity fluctuations excite the thermo-acoustic instabilities of the industrial combustion system, whereas equivalence ratio fluctuations damp them. This result was then used to propose a design change that improves the thermo-acoustic stability behavior of the burner. It was found that the stability of the burner could be improved by shifting the location of the fuel injection downstream, towards the flame. It would not have been possible to identify the position of the fuel injectors as an important design parameter using a SISO model. This analysis therefore demonstrates the additional benefit of MISO models.

\section{What is the performance of the forced response method compared to the self- excited method?}

A self-excited simulation was performed as well for the partially premixed case of the swirl stabilized flame from Chapter 4 . The self-excited method showed a slightly better comparison to experimental data than the forced response method (combination of Flame Describing Functions from CFD and nonlinear stability analysis using the Generalized Instability Model). This was somehow expected, because not only the flow field, but also the acoustics are modeled with a 3D code.

If only one design or operating point is of interest for a certain configuration, the self-excited method is cheaper with respect to CPU time as well (see section 4.8.1). The main advantage of the forced response method, however, is the fact that it directly allows for parameter sweeps. It can for instance be estimated in which direction the flame time lag (and thus the phase of the FDF) changes when the operating point (e.g. equivalence ratio or air preheat temperature) is changed. A stability map as function of the phase of the FDF, such as shown in figure 4.20, can be created within a couple of hours. Also the effect of certain design changes (e.g. a change in combustion chamber length) can be easily assessed. Such variation studies are much more expensive with the self-excited method, since for each operating point a new CFD simulation would be required.

Which approach is most suitable for a certain configuration therefore depends on the question how many operating points or designs need to be tested. 


\subsection{Recommendations}

The limit cycle pressure amplitudes of both generic and industrial test cases were successfully predicted using numerical methods. The methods proposed in this work will hopefully contribute to a more efficient development of clean and high efficiency gas turbine combustors. In this section some recommendations for future work are proposed.

Some of these recommendations have already been mentioned in the previous section, during answering the research questions. However, they are summarized here for completeness.

- The nonlinear thermo-acoustic stability code could be further improved by including additional features. An improvement would be the implementation of branching and conjunction elements in GIM, which would make the complicated modeling strategy that was used in section 4.7.1 unnecessary. Also additional physics such as losses through viscous dissipation may be implemented.

- Investigate if the phase of the FDF can be determined with a higher accuracy. This might be possible by reviewing CFD setup and the combustion modeling. The simulations presented in this thesis were all performed using a relatively simple 1-step combustion model. More advanced combustion models may be able to improve some of the results.

- The Flame Describing Function was determined using harmonic frequency excitation. An initial attempt to determine the Flame Describing Function using multi-frequency excitation was unsuccessful (see Appendix G). It may be possible that other more sophisticated methods may offer a solution here. Multi frequency excitation would make the calculation of the Flame Describing Function much cheaper.

- For the swirl stabilized flame presented in Chapter 4, not only the flame surface was linearly dependent on the tangential component of velocity fluctuations, but the transfer function of the swirler was nonlinearly dependent on the axial component as well. This led to a nonlinear ratio between tangential and axial velocity fluctuation for larger excitation amplitudes. The results suggest that an interesting next step would be to design a (numerical) experiment in which both velocity components can be varied independently, to verify their individual impact on the saturation mechanism of swirl flames. 


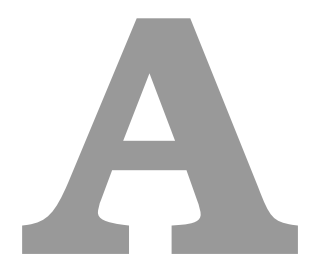

\section{The Transfer Matrix Approach}

Many gas turbine combustion systems can be modeled as a network of one dimensional acoustic elements. In this appendix, a short introduction into acoustic modeling using these one-dimensional network models is given. For each acoustic element, the velocity perturbation $\hat{u}$ and the pressure perturbation $\hat{p}$ at the inlet and the outlet of the element can be coupled by an acoustic transfer matrix. Network models provide a powerful and efficient approach for the acoustic analysis of complex combustion systems. Key references for 1D network models include Hubbard et al. [42], Kim et al. [48], Krüger et al. [57], Polifke et al. [77] and Schuermans et al. [89].

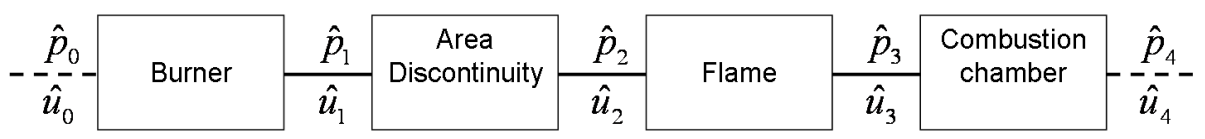

Figure A.1: An example of one-dimensional acoustic network model of a premixed combustion system. Each element can be represented by a transfer matrix $T$, connecting upstream and downstream pressure and velocity. The model may continue upstream of the burner or downstream of the combustion chamber, indicated by the dashed lines.

In figure A.1 an example of an acoustic network is shown, that consists of a burner element, an area discontinuity, a flame element and a combustion chamber element. The acoustic pressure upstream and downstream of each element are given by:

$$
\left(\begin{array}{c}
\hat{p} / \rho_{0} c \\
\hat{u}
\end{array}\right)_{i+1}=\left[\begin{array}{cc}
T_{11} & T_{12} \\
T_{21} & T_{22}
\end{array}\right]\left(\begin{array}{c}
\hat{p} / \rho_{0} c \\
\hat{u}
\end{array}\right)_{i}
$$


Transfer matrices for the different elements can be either determined analytically, numerically or from experiments. In the code available at SIEMENS, usually analytical expressions are used. For the elements that are most frequently used, the transfer matrices will now be given:

\section{Duct element}

Consider a duct with a uniform cross-sectional area, mean pressure $p_{0}$, mean temperature $T_{0}$, mean flow velocity $u_{0}$ and length $L$. The unsteady flow parameters are only a function of the axial spatial coordinate $x$ and time $t$. Then, the linear, one dimensional, homogeneous wave equation (see Chapter 2, equation 2.6) admits a general solution [67]:

$$
\begin{aligned}
& \hat{p}(\omega, x)=p^{+} e^{-i k^{+} x}+p^{-} e^{+i k^{-} x} \\
& \hat{u}(\omega, x)=\frac{1}{\rho_{0} c}\left(p^{+} e^{-i k^{+} x}-p^{-} e^{+i k^{-} x}\right)
\end{aligned}
$$

with $p^{+}$and $p^{-}$the complex amplitudes of the forward and backward traveling waves. The wave number $k^{ \pm}$is given by:

$$
k^{ \pm}=\frac{\omega / c}{1 \pm M a}
$$

with $M a=u_{0} / c$ the Mach number.

From equations A.1, A.2 and A.3 it follows that the transfer matrix of a duct element is:

$$
\left[\begin{array}{ll}
T_{11} & T_{12} \\
T_{21} & T_{22}
\end{array}\right]=\frac{1}{2}\left[\begin{array}{ll}
\left(e^{-i k^{+} L}+e^{+i k^{-} L}\right. & \left(e^{-i k^{+} L}-e^{+i k^{-} L}\right) \\
e^{-i k^{+} L}-e^{+i k^{-} L} & \left(e^{-i k^{+} L}+e^{+i k^{-} L}\right)
\end{array}\right]
$$

\section{Area discontinuity}

An area discontinuity (see Chapter 3 , section D) represents a sudden jump in cross section. The transfer matrix assumes that the pressure and volume flow $(A \cdot \hat{u})$ across the element are constant:

$$
\begin{aligned}
\hat{p}\left(\omega, x_{d}\right) & =\hat{p}\left(\omega, x_{u}\right) \\
A_{d} \hat{u}\left(\omega, x_{d}\right) & =A_{u} \hat{u}\left(\omega, x_{u}\right)
\end{aligned}
$$

Here the $A$ is the area and the sub and superscripts $d$ and $u$ stand for upstream and downstream of the area discontinuity. In its simplest form, the transfer matrix of the area discontinuity not including any mean flow can then be written as:

$$
\left[\begin{array}{cc}
T_{11} & T_{12} \\
T_{21} & T_{22}
\end{array}\right]=\left[\begin{array}{cc}
1 & 0 \\
0 & \frac{A_{u}}{A_{d}}
\end{array}\right]
$$


In reality, when the mean flow is not zero, an additional acoustic reflection or absorption at these discontinuities can be encountered. An analytical description for the transfer matrix of an area discontinuity with mean flow can be derived using the linearized equations for mass and momentum. As shown by Gentemann et al. [32] the transfer matrix for the area discontinuity accounting for mean flow is:

$$
\left[\begin{array}{ll}
T_{11} & T_{12} \\
T_{21} & T_{22}
\end{array}\right]=\left[\begin{array}{cc}
1 & {\left[1-\zeta-\left(\frac{A_{u}}{A_{d}}\right)^{2}\right]_{u}} \\
\frac{A_{u}}{A_{d}} & M_{u}-i \frac{\omega}{c} l_{\text {eff }} \\
-i \frac{\omega}{c} l_{\text {red }}-M_{d} & ]
\end{array}\right.
$$

Here $M$ is the Mach-number accounting for mean flow. The quantities $l_{\text {red }}, l_{\text {eff }}$ are correction factors accounting for the sharp edges of the area discontinuity and $\zeta$ accounts for acoustic losses. Expressions for these factors as well as the derivation of the equations can be found in Gentemann et al. [32].

\section{Branching and conjunction elements}

A branching element splits the network in two sub-passages, whereas a conjunction elements merges two sub-passages into one passage. This is illustrated in figure A.2.

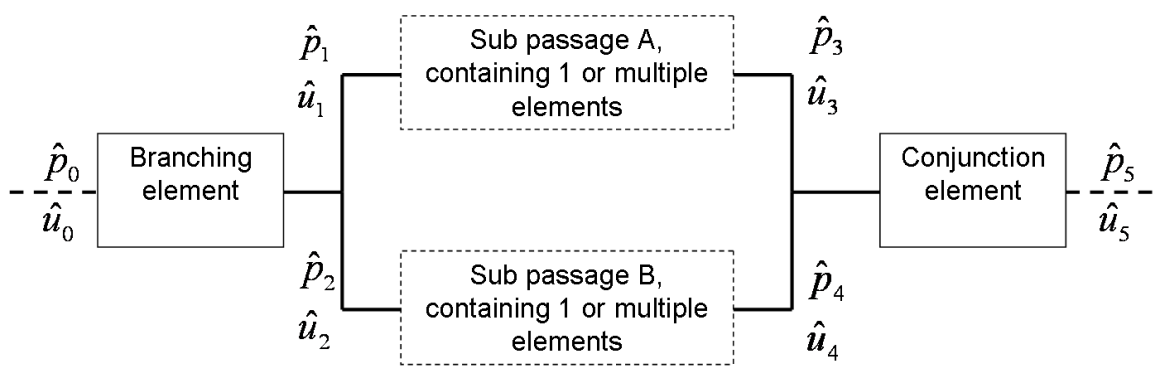

Figure A.2: Branching and conjunction elements.

The transfer matrices of branching and conjunction elements assume that the pressure and the mass flow across the element are constant: For the branching element:

$$
\begin{array}{r}
\hat{p}_{0}(\omega)=\hat{p}_{1}(\omega)=\hat{p}_{2}(\omega) \\
\rho_{0} A_{0}\left(U_{0}+\hat{u}_{0}(\omega)\right)=\rho_{1} A_{1}\left(U_{1}+\hat{u}_{1}(\omega)\right)+\rho_{2} A_{2}\left(U_{2}+\hat{u}_{2}(\omega)\right)
\end{array}
$$

and for the conjunction element:

$$
\begin{array}{r}
\hat{p}_{3}(\omega)=\hat{p}_{4}(\omega)=\hat{p}_{5}(\omega) \\
\rho_{3} A_{3}\left(U_{3}+\hat{u}_{3}(\omega)\right)+\rho_{4} A_{4}\left(U_{4}+\hat{u}_{4}(\omega)\right)+\rho_{5} A_{5}\left(U_{5}+\hat{u}_{5}(\omega)\right)
\end{array}
$$

with $U_{i}$ the mean flow velocity. 


\section{Boundary conditions}

Boundary conditions terminate the acoustic network. An arbitrary impedance can be specified at inlet and exit boundary conditions:

$$
\hat{p}(\omega)=Z(\omega) \hat{u}(\omega)
$$

The most common boundary conditions are of the type $\hat{p}=0$ (meaning $Z=0$ ) or $\hat{u}=0$ (meaning $Z=\infty$ ).

\section{Diffuser element}

A diffuser element (or variable duct element) is a specific type of duct element, in which the diameter, pressure and/or temperature are linearly increasing or decreasing over the element. Such an element is for instance useful to model the passages between the vanes of a swirler, where both the pressure and cross-sectional area are decreasing over the length.

The transfer matrix of the variable duct element is solved by splitting it into $N$ ordinary ducts with constant properties. Typically in the order of $N=100$ increments are used.

$$
\left[\begin{array}{ll}
T_{11} & T_{12} \\
T_{21} & T_{22}
\end{array}\right]=\prod_{i=1}^{N}\left[\begin{array}{ll}
T_{11} & T_{12} \\
T_{21} & T_{22}
\end{array}\right]_{i}
$$

Here the operator $\prod$ stands for multiplication of the matrices.

\section{Flame element}

A thin flame satisfies the following relations (see Kostrzewa [51]):

$$
\begin{aligned}
\hat{p}\left(\omega, x_{d}\right) & =\hat{p}\left(\omega, x_{u}\right) \\
A_{d} \hat{u}\left(\omega, x_{d}\right) & =A_{u} \hat{u}\left(\omega, x_{u}\right)+\frac{\gamma-1}{\rho_{0} c^{2}} \hat{Q}(\omega)
\end{aligned}
$$

with the sub and superscripts $d$ and $u$ standing for upstream and downstream of the flame.

In order to obtain a transfer matrix, a flame model for $\hat{Q}$ has to be specified, which relates heat release fluctuations to velocity and pressure fluctuations. The specific form of the transfer matrix depends on the flame model for $\hat{Q}$ that is used. For perfectly premixed systems, a SISO model can be used (for instance Chapter 1, equation 1.4) and the transfer matrix would be of size $2 x 2$. When, for partially premixed systems, a MISO model is used (e.g. equation 1.9), the transfer matrix would have velocities and pressures at different locations as input and thus have a larger size. The standard flame model that is usually used in the Transfer Matrix Approach at SIEMENS 
is (see Ref. [51]):

$$
\begin{aligned}
\frac{\hat{Q}(\omega)}{Q_{0}}= & -\underbrace{\left[\frac{\hat{p}_{\text {inj }} e^{-i \omega \tau_{\text {inj }}}}{2\left(p_{\text {fuelline }}^{0}-p_{i n j}^{0}\right)}+\frac{\hat{u}_{i n j} e^{-i \omega \tau_{\text {inj }}}}{u_{i n j}^{0}}+\frac{\hat{p}_{i n j} e^{-i \omega \tau_{i n j}}}{\rho_{0} c^{2}}\right]}_{\text {Equivalence ratio fluctuations }} \\
& +\underbrace{\frac{\hat{u}_{\text {burner }} e^{-i \omega \tau_{\text {burner }}}}{u_{\text {burner }}^{0}}}_{\text {Velocity fluctuations burner exit }}+\underbrace{\frac{\hat{p}_{\text {flame }}}{\rho_{0} c^{2}}}_{\text {Pressure fluctuations at flame }}
\end{aligned}
$$

A sketch of a geometry to which this flame model applies and the corresponding acoustic network are shown in figure A.3. As shown in figure A.3(b), the flame model has 6 inputs ( 3 pressures and 3 velocities) and 2 outputs ( 1 pressure and 1 velocity). When equation A.18 is inserted into equation A.17, a transfer matrix of size $2 x 6$ can be derived.

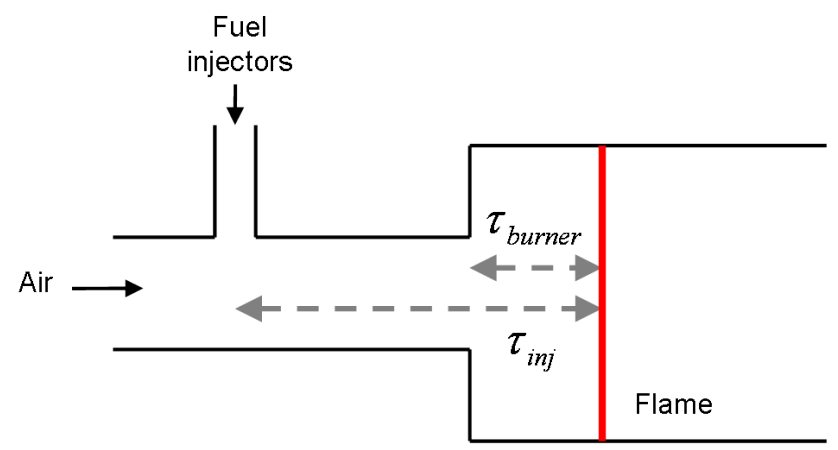

(a) Sketch of geometry.

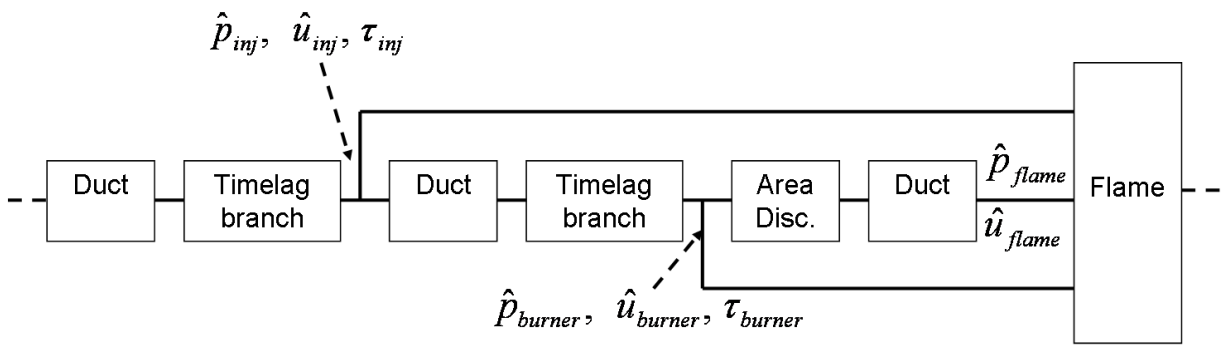

(b) Corresponding TMA Model.

Figure A.3: Example of a configuration for a partially premixed system, featuring an 1D flame and separate air and fuel inlets.

\section{Solution procedure}

The values of the pressure and velocity at each node of the network can be stored in the solution vector $\mathbf{X}$. The governing equations for each element in the network can 
then be rewritten as follows:*

$$
\underbrace{\left[\begin{array}{ccccccccc}
1 & -Z_{\text {inlet }} & 0 & 0 & \cdots & 0 & 0 & 0 & 0 \\
-T_{11}^{0} & -T_{12}^{0} & 1 & 0 & \cdots & 0 & 0 & 0 & 0 \\
-T_{21}^{0} & -T_{22}^{0} & 0 & 1 & \cdots & 0 & 0 & 0 & 0 \\
0 & 0 & -T_{11}^{1} & -T_{12}^{1} & \cdots & 0 & 0 & 0 & 0 \\
0 & 0 & -T_{21}^{1} & -T_{22}^{1} & \cdots & 0 & 0 & 0 & 0 \\
\vdots & \vdots & \vdots & \vdots & \ddots & \vdots & \vdots & \vdots & \vdots \\
0 & 0 & 0 & 0 & \cdots & -T_{11}^{N} & -T_{12}^{N} & 1 & 0 \\
0 & 0 & 0 & 0 & \cdots & -T_{21}^{N} & -T_{22}^{N} & 0 & 1 \\
0 & 0 & 0 & 0 & \cdots & 0 & 0 & 1 & -Z_{\text {exit }}
\end{array}\right]}_{\mathbf{A}(\omega)} \underbrace{\left(\begin{array}{c}
\hat{p}_{\text {inlet }} \\
\hat{u}_{\text {inlet }} \\
\hat{p}_{0} \\
\hat{u}_{0} \\
\vdots \\
\hat{p}_{N} \\
\hat{u}_{N} \\
\hat{p}_{\text {exit }} \\
\hat{u}_{\text {exit }}
\end{array}\right)}_{\mathbf{X}}=\left(\begin{array}{c}
0 \\
0 \\
0 \\
0 \\
0 \\
\vdots \\
0 \\
0 \\
0
\end{array}\right)
$$

The complex eigen frequencies $\omega$ are the eigenvalues of the system matrix $A(\omega)$ and can be determined by solving:

$$
\operatorname{Det}(A(\omega))=0
$$

Here $\omega=\omega_{r}+i \omega_{i}$, with the real part $\omega_{r}$ the excited frequency. The imaginary part $\omega_{i}$ determines the growth or damping of the acoustic mode and is related to the growth rate by $\Gamma=-2 \pi \omega_{i}$.

\section{Possible strategy for nonlinear extension}

When a nonlinear flame model is used, the heat release model is dependent on the velocity amplitude: $\hat{Q}=\hat{Q}(\omega, \hat{u})$. In this case, the solution matrix $A$ becomes a function of $\hat{u}$ as well. Recently, Palies et al. [72] introduced a 1D frequency domain model that solves for real valued eigenfrequencies $\omega=\omega_{r}$ :

$$
\operatorname{Det}(A(\omega, \hat{u}))=0 \quad \text { with } \quad \omega_{i}=0
$$

This equation can be solved by imposing the additional restriction $\omega_{i}=0$. More information about the algorithm to solve this equation, as well as the application of this method to nonlinear thermo-acoustic problems, can be found in the work of Palies. Incorporating this method in the Transfer Matrix Approach has not been performed within this thesis, but it would be interesting to investigate this in future work.

*The third and fourth row of equation A.19 actually read:

$$
\begin{array}{ll}
-T_{11}^{1} \cdot \hat{p}_{0} & -T_{12}^{1} \cdot \hat{u}_{0}+1 \cdot \hat{p}_{1}=0 \\
-T_{21}^{1} \cdot \hat{p}_{0} & -T_{22}^{1} \cdot \hat{u}_{0}+1 \cdot \hat{u}_{1}=0
\end{array}
$$




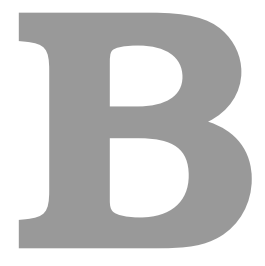

\section{Generalized Instability Model: derivation of forcing term}

In this appendix the derivation of equation 2.11 and 2.12 of Chapter 2 is discussed in more detail. The derivation is very similar to Culick and Yang [18-21].

First the wave equation for the acoustic pressure (equation 2.6) is multiplied by the $m^{t h}$ mode shape $\Psi_{m}$ and the Helmholtz equation (equation 2.10) is multiplied by the acoustic pressure $p^{\prime}$. Then the results are subtracted, leading to:

$$
\Psi_{m} \nabla^{2} p^{\prime}-p^{\prime} \nabla^{2} \Psi_{m}-\frac{1}{c^{2}} \frac{\partial^{2} p^{\prime}}{\partial t^{2}} \Psi_{m}-k_{m}^{2} \Psi_{m} p^{\prime}=h \Psi_{m}
$$

The index $m$ has been used here, to distinguish it from the index $n$, which is summed over all modes. Multiplying the result by $-c^{2}$, noting that $\omega_{m}=k_{m} c$, and integrating over the volume leads to:

$$
-\int_{V} c^{2}\left[\Psi_{m} \nabla^{2} p^{\prime}-p^{\prime} \nabla^{2} \Psi_{m}\right] d V+\int_{V} \frac{\partial^{2} p^{\prime}}{\partial t^{2}} \Psi_{m} d V+\int_{V} \omega_{m}^{2} \Psi_{m} p^{\prime} d V=-\int_{V} c^{2} h \Psi_{m} d V
$$

The next step is first using the identity $a \nabla b=\nabla(a b)-b \nabla a$ on the first integral of this 
expression, which leads (after some re-arranging) to equation B.3:

$$
\begin{aligned}
\int_{V} \frac{\partial^{2} p^{\prime}}{\partial t^{2}} \Psi_{m} d V+\int_{V} \omega_{m}^{2} \Psi_{m} p^{\prime} d V & =-\int_{V} c^{2} h \Psi_{m} d V \\
& +\int_{V} c^{2}\left[\nabla \cdot\left(\Psi_{m} \nabla p^{\prime}\right)-\nabla \cdot\left(p^{\prime} \nabla \Psi_{m}\right)\right] d V \\
& +\int_{V} c^{2}\left[-\nabla \Psi_{m} \cdot \nabla p^{\prime}+\nabla p^{\prime} \cdot \nabla \Psi_{m}\right] d V
\end{aligned}
$$

Here the terms on the third line of equation B.3 cancel each other. Next the divergence theorem $\left(\int_{V}(\nabla \cdot \mathbf{F}) d V=\int_{\partial V}(\mathbf{F} \cdot \mathbf{n}) d A\right)$ is used:

$$
\begin{aligned}
\int_{V} \frac{\partial^{2} p^{\prime}}{\partial t^{2}} \Psi_{m} d V+\int_{V} \omega_{m}^{2} \Psi_{m} p^{\prime} d V & =-\int_{V} c^{2} h \Psi_{m} d V \\
& +\int_{\partial V} c^{2}\left[\Psi_{m} \nabla p^{\prime}-p^{\prime} \nabla \Psi_{m}\right] \cdot \mathbf{n} d A \\
& =-\int_{V} c^{2} h \Psi_{m} d V \\
& -\int_{\partial V} c^{2}\left[\Psi_{m} f_{0}+f_{1} \nabla \Psi_{m}\right] \cdot \mathbf{n} d A
\end{aligned}
$$

In which $f_{0}=-\nabla p^{\prime}$ and $f_{1}=p^{\prime}$ at the boundaries.

Now equation 2.8 can be substituted for $p^{\prime}$ :

$$
\begin{aligned}
& \int_{V} p_{0} \sum_{n=1}^{N} \Psi_{n} \ddot{\eta}_{n} \Psi_{m} d V+\int_{V} \omega_{m}^{2} \Psi_{m} p_{0} \sum_{n=1}^{N} \Psi_{n} \eta_{n} d V \\
= & p_{0} \sum_{n=1}^{N} \ddot{\eta_{n}} \int_{V} \Psi_{m} \Psi_{n} d V+p_{0} \sum_{n=1}^{N} \omega_{m}^{2} \eta_{n} \int_{V} \Psi_{m} \Psi_{n} d V=-F_{m}
\end{aligned}
$$

Here $F_{m}$ is the right hand side of equation B.5. This can be written as the following system:

$$
\{\ddot{\eta}\}=[E]^{-1}[W][E]\{\eta\}=-\frac{1}{p_{0}}[E]^{-1}\{F\}
$$

with

$$
\begin{aligned}
E_{m n} & =\int_{V} \Psi_{m} \Psi_{n} d V \\
W_{m n} & =\omega_{m}^{2} \delta_{m n}
\end{aligned}
$$

Generally, equation B.7 is a coupled set of equations, because the eigenmodes are usually not orthogonal. However, if the modes are orthogonal, $E_{m n}=0$ if $m \neq n$. Physically, this coupling of modes represents the possible transfer of energy from 
one mode to another mode. Key references on mode coupling include Ref $[7,68]$. The importance of mode coupling may depend from case to case and usually occurs if there is more than one unstable mode. In case there is just one dominant mode, the phenomenon can usually be neglected. This was shown by Balachandran et al. [5].

The current implementation of the code is limited to cases in which this mode coupling does not occur. In cases without mode coupling, each mode can be considered individually and equation B.7 can be simplified to:

$$
\begin{aligned}
\ddot{\eta}_{n}+\omega_{n}^{2} \eta_{n} & =-\frac{1}{p_{0} \int_{V} \Psi_{n}^{2} d V} F_{n}(\eta, \dot{\eta}, \ddot{\eta}) \\
F_{n}(\eta, \dot{\eta}, \ddot{\eta}) & =\underbrace{-\int_{V} c^{2} h \Psi_{n} d V}_{\text {Mean flow \& heat release (eq. 2.7) }}+\underbrace{\oint_{\partial V} c^{2}\left[\Psi_{n} f_{0}+f_{1} \nabla \Psi_{n}\right] \cdot \mathbf{n} d A}_{\text {Boundary conditions }}
\end{aligned}
$$

This result is identical to equation 2.11 and 2.12. 


\section{C \\ LODI Equations}

To avoid acoustic reflections, the simulations presented in this thesis have been performed with non reflecting boundary conditions. The implementation is based on the LODI approach (Local One-Dimensional Inviscid). In this Appendix the specific equations solved in the LODI approach are given.

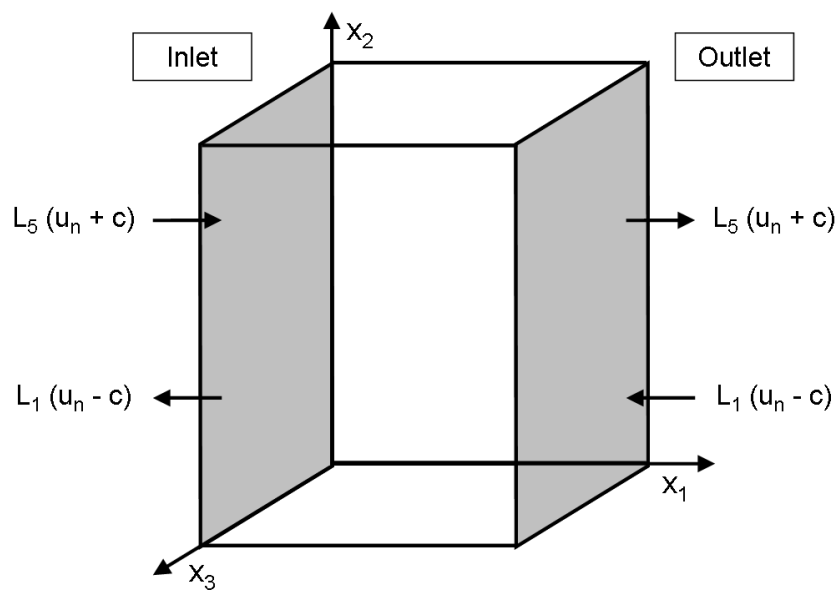

Figure C.1: Boundary conditions located on the $x_{1}$-axis. [75]

In the LODI approach (Polifke [78], Poinsot [75]), disturbances are separated into waves traveling into the domain and out of the domain. In figure C.1 a simple geometry with an inlet and an outlet is shown. In this figure $L_{5}$ is the characteristic wave traveling in the positive $x_{1}$-direction (with characteristic speed $u_{n}+c$ ) and $L_{1}$ the 
characteristic wave traveling in the negative $x_{1}$-direction (with characteristic speed $\left.u_{n}-c\right)$.

According to Poinsot [78] the one-dimensional Euler equations at the boundary condition are:

$$
\begin{aligned}
\frac{\partial p}{\partial t}+\frac{1}{2}\left(L_{5}+L_{1}\right) & =0 \\
\frac{\partial u_{n}}{\partial t}+\frac{1}{2 \rho c}\left(L_{5}-L_{1}\right) & =0
\end{aligned}
$$

where $u_{n}$ is the velocity normal to the boundary condition and $x_{n}$ the coordinate normal to the boundary condition.

In the next sections, expressions for $L_{1}$ and $L_{5}$ will be derived for the following cases:

- Inflow boundary condition without excitation (section C.1)

- Inflow boundary condition with excitation (section C.2)

- Outflow boundary conditon (section C.3)

\section{C.1 Inflow boundary condition without excitation}

A perfectly non reflecting inlet boundary condition could be achieved by setting the incoming wave $\left(L_{5}\right)$ to zero. However, when acoustic waves from inside the computational domain $\left(L_{1}\right)$ hit the boundary condition, the velocity cannot remain constant if a reflection is to be avoided. Regardless of instantaneous fluctuations of the inlet velocity, the time average inlet velocity should be close to a reference value. A perfectly non reflecting boundary condition would not allow this mean flow control. Therefore, an artificial wave $\left(L_{5, \text { relax }}\right)$ is sent into the domain. The wave amplitude of $L_{5, \text { relax }}$ is zero if the value of the velocity at the boundary condition is identical to the reference value $u_{\infty}$. The waves $L_{1}$ and $L_{5}$ are defined as:

$$
\begin{aligned}
L_{1} & =\left(u_{n}-c\right)\left(\frac{\partial p}{\partial x_{n}}-\rho c \frac{\partial u_{n}}{\partial x_{n}}\right) \\
L_{5} & =L_{5, \text { exact }}+L_{5, \text { relax }} \\
L_{5, \text { exact }} & =0 \\
L_{5, \text { relax }} & =\rho c \sigma \frac{c}{L}\left(u_{n}-u_{\infty}\right)=\frac{2 \rho c}{\tau}\left(u_{n}-u_{\infty}\right)
\end{aligned}
$$

with

$$
\tau=\frac{2 L}{\sigma c}
$$

Inserting equations C.3 to C.6 into equations C.1 and C.2 leads to the final equations that are solved at the inlet boundary condition: 


$$
\begin{aligned}
& \frac{\partial p}{\partial t}+\frac{1}{2}\left(u_{n}-c\right)\left(\frac{\partial p}{\partial x_{n}}-\rho c \frac{\partial u_{n}}{\partial x_{n}}\right)+\frac{\rho c}{\tau}\left(u_{n}-u_{\infty}\right)=0 \\
& \frac{\partial u_{n}}{\partial t}-\frac{1}{2 \rho c}\left(u_{n}-c\right)\left(\frac{\partial p}{\partial x_{n}}-\rho c \frac{\partial u_{n}}{\partial x_{n}}\right)+\frac{u_{n}-u_{\infty}}{\tau}=0
\end{aligned}
$$

Here the parameter $\tau$ (unit $s$ ) is the time scalar, which is a relaxation coefficient. The boundary condition now behaves like a low pass filter, with its cut-off frequency depending on the magnitude of $\tau$. In the limiting case of $\tau \rightarrow 0$, equations C.8 and C.9 simply converge to $u_{n}(t)=u_{\infty}$, where as for $\tau \rightarrow \infty$ the equations converge to $\frac{\partial p}{\partial t}=-\rho c \frac{\partial u_{n}}{\partial t}$, which is the exact expression for a wave traveling in negative $x_{n}$ direction (out of the domain).

In Chapter 3, figure 3.4, the reflection coefficient as a function of frequency and the time scalar was shown.

\section{C.2 Inflow boundary condition with excitation}

A non reflective inlet boundary condition behavior is of significant importance for performing forced simulations. If a forced inlet condition is located upstream of an area contraction, it is possible that between the inlet condition and the area contraction acoustic energy can accumulate in case a reflecting boundary condition is used. This acoustic energy accumulation will ultimately result in characteristic wave amplitudes of unphysical magnitude, causing the CFD solver to fail. Thus, a non reflecting inlet boundary condition was used, which allows prescribing perturbations.

The ingoing prescribed wave $g$ is included in the ingoing characteristic wave $L_{5, \text { relax }}$ :

$$
L_{5, \text { relax }}=\frac{2 \rho c}{\tau}\left(u_{n}-u_{\infty}^{0}+g\right)+\rho c \frac{\partial g}{\partial t}
$$

with

$$
g=\frac{1}{2}(\underbrace{\frac{p^{\prime}}{\rho c}}_{=0}-u^{\prime})
$$

Here $u_{\infty}^{0}$ is the mean far stream velocity. For exciting a simulation, it is not strictly necessary to perturb the pressure. In the current implementation, only the velocity is excited. It can be seen that $p^{\prime}=0$ in equation C.11. The non reflective inlet boundary condition requires the velocity perturbation $u^{\prime}$ to be continuously differentiable. For this reason, a superposition of harmonic excitations (sinus overlay) is used in the code. This signal contains $N$ frequencies between $f_{\text {low }}$ and $f_{\text {max }}$. The perturbation can be written as: 


$$
\begin{array}{r}
g=-\frac{A}{2} u_{\infty}^{0} \sum_{i=1}^{N} \sin 2 \pi f_{i} t \\
u_{\infty}(t)=u_{\infty}^{0}-2 g=u_{\infty}^{0}\left(1+A \sum_{i=1}^{N} \sin 2 \pi f_{i} t\right)
\end{array}
$$

where $A$ is the amplitude of the perturbation. In case $N=1$, the signal is a pure single harmonic wave. This equation is identical to equation 3.40 of Chapter 3.

\section{C.3 Outflow boundary conditon}

The non reflecting outlet formulation is derived similar to the inlet boundary condition:

$$
\begin{aligned}
L_{5} & =\left(u_{n}+c\right)\left(\frac{\partial p}{\partial x_{n}}+\rho c \frac{\partial u_{n}}{\partial x_{n}}\right) \\
L_{1} & =L_{1, \text { exact }}+L_{1, \text { relax }} \\
L_{1, \text { exact }} & =0 \\
L_{1, \text { relax }} & =\frac{\sigma\left(1-M^{2}\right) c}{L}\left(p-p_{\infty}\right)=\frac{2\left(1-M^{2}\right)}{\tau}\left(p-p_{\infty}\right)
\end{aligned}
$$

Leading to:

$$
\begin{array}{r}
\frac{\partial p}{\partial t}+\frac{1}{2}\left(u_{n}+c\right)\left(\frac{\partial p}{\partial x_{n}}+\rho c \frac{\partial u_{n}}{\partial x_{n}}\right)+\frac{\left(1-M^{2}\right)}{\tau}\left(p-p_{\infty}\right)=0 \\
\frac{\partial u_{n}}{\partial t}+\frac{1}{2 \rho c}\left(u_{n}+c\right)\left(\frac{\partial p}{\partial x_{n}}+\rho c \frac{\partial u_{n}}{\partial x_{n}}\right)-\frac{\left(1-M^{2}\right)}{\rho c \tau}\left(p-p_{\infty}\right)=0
\end{array}
$$

Here $M$ is the Mach-number and $p_{\infty}$ the reference pressure. In the limiting case of $\tau \rightarrow 0$, equations C.18 and C.19 simply converge to $p(t)=p_{\infty}$, where as for $\tau \rightarrow \infty$ the equations converge to $\frac{\partial p}{\partial t}=+\rho c \frac{\partial u_{n}}{\partial t}$, which is the exact expression for a wave traveling in positive $x_{n}$-direction (out of the domain). 


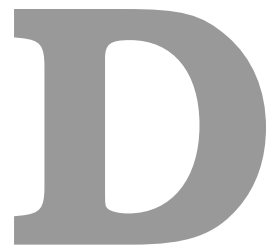

\section{Transfer function of an area discontinuity}

Ducts with a sudden area change are a representative feature of combustion systems. The duct with sudden change of cross sectional area is utilized as a test case for the validation of the CFD code and methods, because the acoustic transfer matrices are well known from theory, experiments and simulations with a Finite Element acoustic code. The same study has been carried out before by Kostrzewa [51], using the CFD code Ansys CFX 10.0.

In figure D.1 a sketch of the considered geometry is shown.

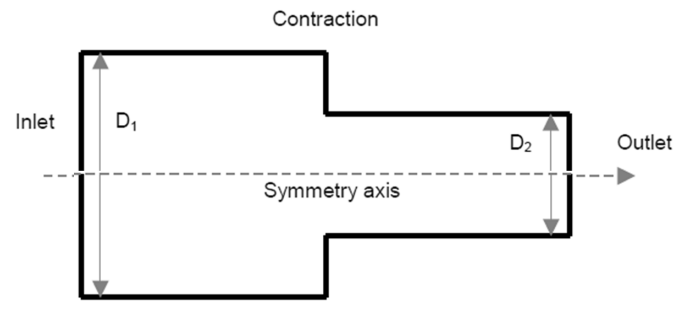

(a) Geometry

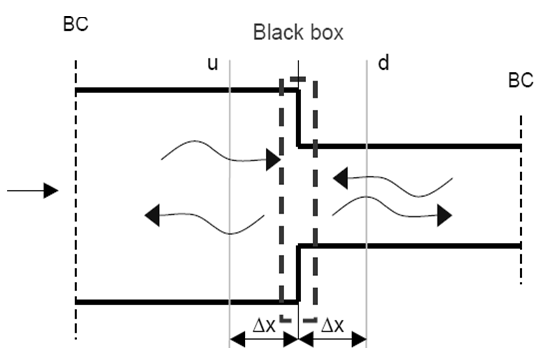

(b) Sampling planes

Figure D.1: Geometry of the area discontinuity and location of sampling planes. Pictures taken from Kostrzewa [51].

The inlet diameter $D_{1}=0.2 \mathrm{~m}$ and is contracted to $D_{2}=0.104 \mathrm{~m}$. The total length of the geometry is $0.8 \mathrm{~m}$. The boundary conditions at the inlet were a mean velocity $u_{0}$ of $0.7 \mathrm{~m} / \mathrm{s}$ and a static temperature of $293 \mathrm{~K}$. At the outlet an average static 
pressure of 1 bar was applied.

\section{Analytical model}

An analytical description for the transfer matrix of an acoustic element can be derived using the linearized equations for mass and momentum. If an acoustic wave length $\lambda$ is much larger than the characteristic length $L$ of this element $(\lambda>>L)$, this element can be treated as an acoustically compact element. As shown by Gentemann et al. [32] the transfer matrix $\mathbf{T}$ for the area discontinuity is:

$$
\left(\begin{array}{c}
\hat{p} / \rho_{0} c \\
\hat{u}
\end{array}\right)_{d}=\left[\begin{array}{ll}
T_{11} & T_{12} \\
T_{21} & T_{22}
\end{array}\right]\left(\begin{array}{c}
\hat{p} / \rho c \\
\hat{u}
\end{array}\right)_{u}
$$

with

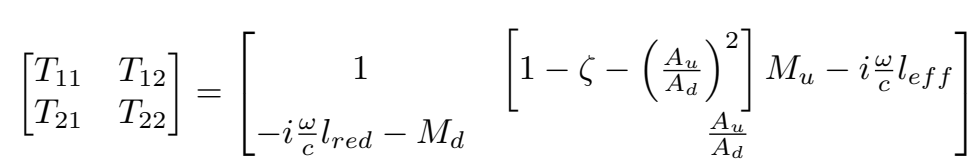

Here the sub and superscripts $d$ and $u$ stand for upstream and downstream of the area discontinuity. The parameter $M$ is the Mach-number accounting for mean flow and $A$ the area of the duct. The quantities $l_{\text {red }}, l_{\text {eff }}$ are correction factors accounting for the sharp edges of the area discontinuity and $\zeta$ accounts for acoustic losses. Expressions for these factors as well as the derivation of the equations can be found in Gentemann et al. [32].

\section{CFD Model}

The problem has been simulated on a 2D axisymmetric mesh, consisting of 19750 hexahedral elements. First a steady state solution was obtained. The axial velocity distribution is shown in figure D.2. It can be seen that the velocity upstream of the contraction is fairly evenly distributed over the radius, but downstream of the area discontinuity this is not the case. This shows that good averaging techniques (as discussed before in section 3.3.2) are required for the identification of the transfer function.

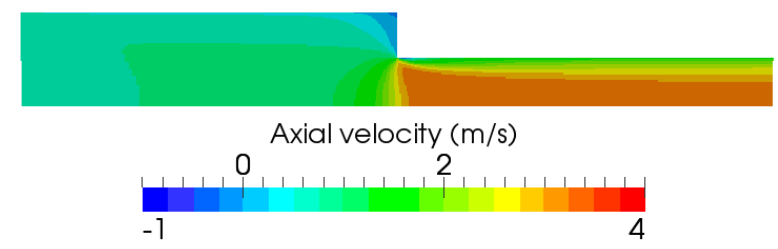

Figure D.2: Steady state axial velocity.

Next, the velocity at the inlet was excited using the non-reflecting sinus overlay boundary condition (equation 3.40). The signal contained all frequencies between 50 $\mathrm{Hz}$ and $2000 \mathrm{~Hz}$ in intervals of $2 \mathrm{~Hz}$. It has been determined from figure 3.4 that for this case a value of the time scalar $\tau=0.03$ gives a good optimum between small deviation of the mean values from the far field properties and small reflection in 
the frequency range of interest. All frequencies larger than $50 \mathrm{~Hz}$ are reflected less than $20 \%$. The parameter $A$ was determined in such a way that the amplitude of the velocity fluctuation did not exceed $10 \%$ of the mean flow. This $10 \%$ excitation was recommended by Gentemann [32] and Kostrzewa [51], to have a good signal to noise ratio but prevent nonlinear effects.

It should be noted that Kostrzewa perturbed both his inlet and outlet boundary condition with white noise. Non-reflective boundary conditions were unavailable for his research and he found that system identification with reflective boundary conditions is most successful with white noise excitation.

The locations of the sampling planes are shown in figure D.1(b). The planes are located at $\Delta x=0.05 \mathrm{~m}$ upstream and downstream of the area discontinuity.

The physical length of the transient simulation was chosen to be $0.25 \mathrm{~s}$ and a time step of $d t=5 \cdot 10^{-6} s$ was used, which resulted in $C F L=0.7$.

\section{Postprocessing}

Time series of the acoustic velocity and pressure fluctuations upstream and downstream of the area discontinuity have been sampled. Because this system is a MultipleInput, Multiple-Output (MIMO) System and broadband excitation was used, the Transfer function has been determined using the Wiener Hopf Transformation (see Appendix E).

It should be noticed that the resulting transfer matrix $\mathbf{T}_{C F D}$ is not the transfer matrix $\mathbf{T}_{A D}$ of the area discontinuity, but the transfer matrix of the whole section between the sampling planes (see figure D.1(b)). Therefore, the transfer matrix has to be corrected by the (known) transfer matrices of two simple ducts (with length $\Delta x$ being the distance between the area contraction and the sampling planes):

$$
\mathbf{T}_{A D}=\mathbf{T}_{\text {duct }, \text { upstream }}^{-1} \cdot \mathbf{T}_{C F D} \cdot \mathbf{T}_{\text {duct, downstream }}
$$

with

$$
\mathbf{T}_{\text {duct }}=\frac{1}{2}\left[\begin{array}{ll}
\left(e^{-\frac{i}{1+M a} \frac{\omega \Delta x}{c}}+e^{\frac{i}{1-M a} \frac{\omega \Delta x}{c}}\right. & \left(e^{-\frac{i}{1+M a} \frac{\omega \Delta x}{c}}-e^{\frac{i}{1-M a} \frac{\omega \Delta x}{c}}\right) \\
\left(e^{-\frac{i}{1+M a} \frac{\omega \Delta x}{c}}-e^{\frac{i}{1-M a} \frac{\omega \Delta x}{c}}\right) & \left(e^{-\frac{i}{1+M a} \frac{\omega \Delta x}{c}}+e^{\frac{i}{1-M a} \frac{\omega \Delta x}{c}}\right)
\end{array}\right]
$$

\section{Results}

The results obtained with OpenFoam have been compared against the following reference data:

- Measurements. The pressures and velocities upstream and downstream of the discontinuity were measured using the multi-microphone method. The setup was excited by a siren. More information on the experimental setup and the post processing methods can be found in Gentemann et al. [32].

- Theory. The analytical transfer matrix was calculated according to equation D.2. 
- Finite Element Computation (FEM). More information about this calculation can be found in Pankiewitz et al. [73].

- Reference CFD data by Kostrzewa [51]. The CFD calculations were performed with URANS, using Ansys CFX 10.0. More details can be found in his PhD Thesis [51].

The calculated transfer matrices are presented in figure D.3. The following can be observed:

- For most components of $\mathbf{T}$, there are some discrepancies between the measurement results and all analytical and numerical approaches. This is especially the case for frequencies below $250 \mathrm{~Hz}$. Kostrzewa accounted this to measurement uncertainties [51]. Because the boundary conditions of the experimental setup are partly reflecting, some noise of the surroundings could be influencing the results. Therefore, for this validation case, the comparison against analytical and numerical approaches is more important.

- Element $T_{11}$ represents the ratio between the acoustic pressure upstream and downstream of the area discontinuity. The absolute value corresponds very well to the reference data. The phase of $T_{11}$ also agrees well with all reference data and is slightly closer to the measurement data for larger frequencies than the CFX data.

- Element $T_{12}$ represents the ratio between the downstream acoustic pressure and the upstream acoustic velocity. It agrees particularly well with the CFX data. It corresponds better to the measurements than theory, but the values are slightly larger.

- Element $T_{21}$ connects the acoustic velocity downstream of the area discontinuity to the acoustic pressure upstream. It can be seen that a absolute value larger than zero was measured, while in all numerical and analytical approaches it is very close to zero. Large disagreements between the CFD approaches (both CFX + URANS and OpenFoam + LES) and the other approaches can be seen for the phase of $T_{21}$. A small signal to noise ratio may be the reason for the discrepancies. It should be noticed however that the small absolute values of the element $T_{21}$ makes the prediction of the phase much less important.

- Finally element T22 represents the ratio of acoustic velocity upstream and downstream of the area discontinuity. For this component, both the absolute value and the phase are perfectly predicted and are practically equal to all numerical reference data. The measurement data lies slightly below the numerical data but shows a good fit nonetheless.

Looking at all plots in figure D.3, the results are generally very good. The validation study for the area discontinuity clearly shows that the CFD code and methods presented in this chapter can accurately predict transfer functions and is therefore suited for application to more complicated geometries (which will be done in the next chapters). 

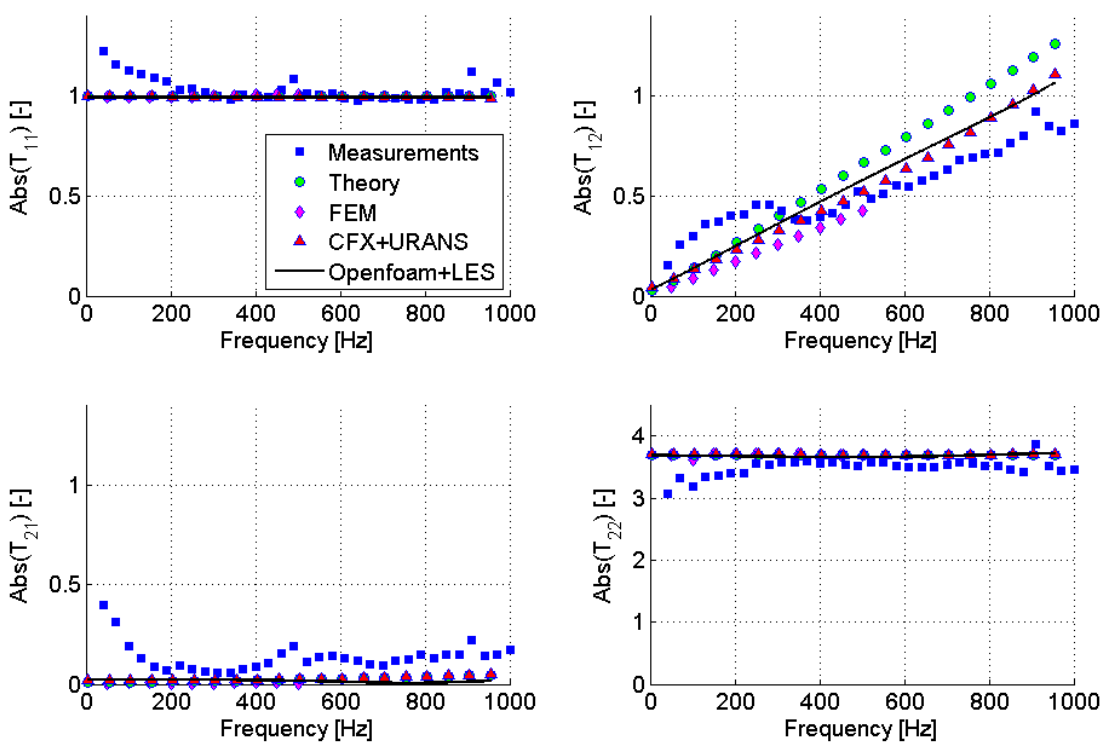

(a) Magnitude
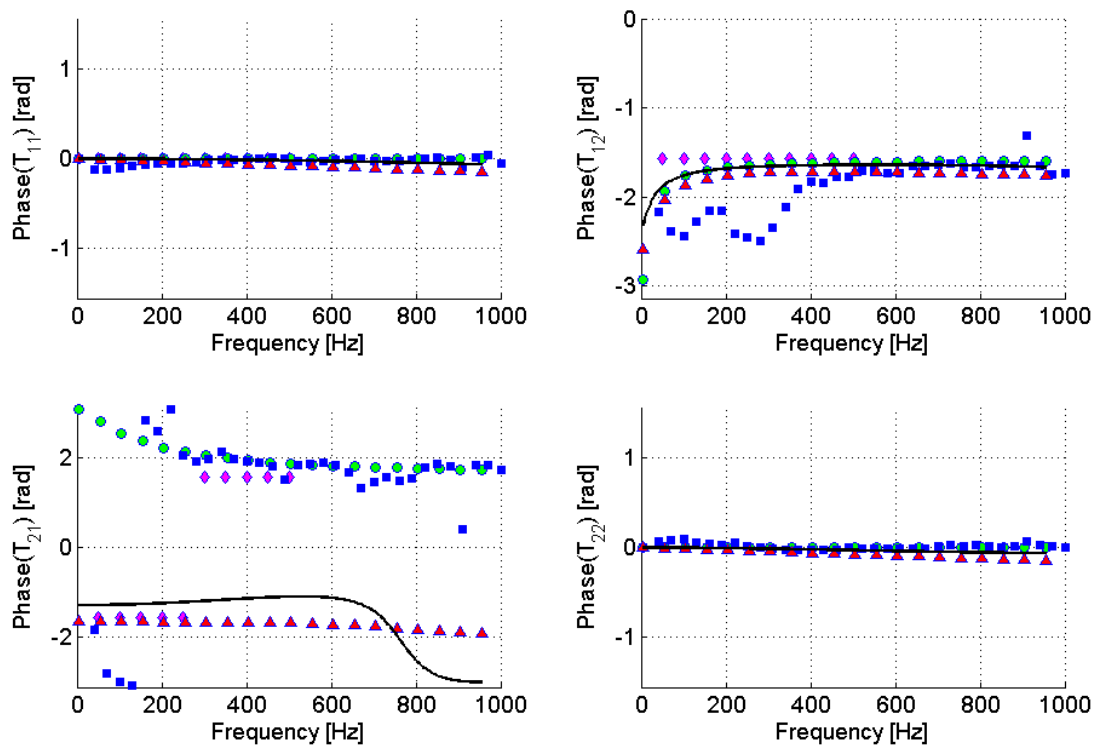

(b) Phase

Figure D.3: Calculated transfer matrix elements with OpenFoam (solid black line), compared against various reference data. 


\section{2 The Wiener Hopf Transformation}

The Wiener Hopf Transformation is a numerical method to reconstruct the transfer function between two signals that have a broadband frequency range. The idea of using the Wiener Hopf Transformation for the post-processing of CFD results was originally introduced by Polifke [76]. A complete mathematical description of the reconstruction procedure can be found in this reference. A brief summary will be given in this appendix.

Let $s(t)$ be a discrete input signal to a given system and $r(t)$ its response. If the system is a Linear Time Invariant (LTI) system, the relation between signals and responses can be written as:

$$
r(t)=\sum_{k=-\infty}^{\infty} h(k) s(t-k)
$$

The factor $h(k)$ is called the unit response vector here. As described by Kostrzewa in his $\mathrm{PhD}$ thesis [51], for some cases it may be hard to define which variable is an input and which variable is a response. In many systems, acoustic waves are both travelling upstream and downstream at the same time. However, as Kostrzewa describes, for acoustic identification in pu-notation it actually does not matter which signal is defined to be the input and which as the response, as long as the non-causal formulation is applied. In the non-causal formulation, the systems' response on a given time $t$ depends both on future and past signals. This in contrast to the causal formulation, in which the response only depends on past signals.

As can be seen in equation E.1, an infinite number of coefficients $h(k)$ should be used to completely determine the frequency response. In practice, an unsteady computation has a finite number of time steps $N$. The system should then be characterized by a finite number of unit impulse responses $L$ (which will be defined in the 
next section). Taking this into account, equation E.1 becomes:

$$
\begin{aligned}
\text { Non-causal formulation: } & r(t) & =\sum_{k=-L}^{L} h(k) s(t-k) \\
\text { Causal formulation: } & r(t) & =\sum_{k=0}^{L} h(k) s(t-k)
\end{aligned}
$$

The Wiener Hopf Transformation is a mathematical method to determine the coefficients $h(k)$ by minimizing the least mean square error. It solves the following equation:

$$
[\boldsymbol{\Gamma}]\{\mathbf{h}\}=\{\mathbf{c}\}
$$

with $\boldsymbol{\Gamma}$ the autocorrelation matrix, $\mathbf{h}$ the unit response vector and $\mathbf{c}$ the crosscorrelation vector. For the non-causal formulation, the autocorrelation matrix is of size $[2 \mathrm{~L}+1 \times 2 \mathrm{~L}+1]$ and is defined as:

$$
[\boldsymbol{\Gamma}]=\frac{1}{N-2 L-1} \sum_{k=L}^{N-L}\left[\begin{array}{ccc}
s(k+L) s(k+L) & \ldots & s(k+L) s(k-L) \\
\vdots & \ddots & \vdots \\
s(k-L) s(k+L) & \ldots & s(k-L) s(k-L)
\end{array}\right]
$$

The components of the cross-correlation vector $\mathbf{c}$ are:

$$
c_{i}=\frac{1}{N-2 L-1} \sum_{k=L}^{N-L} r(k) s(k-i) \quad i=-L \ldots L
$$

Here $N$ is the length of the signals / responses vector (number of time steps).

The approach can also be extended to a Multiple Input, Multiple Output (MIMO) system. For instance, for a system with 2 inputs and 2 outputs, the equations can be rewritten as:

$$
\left[\begin{array}{ll}
{[\boldsymbol{\Gamma}]\left(s_{1}, s_{1}\right)} & {[\boldsymbol{\Gamma}]\left(s_{1}, s_{2}\right)} \\
{[\boldsymbol{\Gamma}]\left(s_{2}, s_{1}\right)} & {[\boldsymbol{\Gamma}]\left(s_{2}, s_{2}\right)}
\end{array}\right]\left[\begin{array}{ll}
\{\mathbf{h}\}_{11} & \{\mathbf{h}\}_{12} \\
\{\mathbf{h}\}_{21} & \{\mathbf{h}\}_{22}
\end{array}\right]=\left[\begin{array}{ll}
\{\mathbf{c}\}\left(r_{1}, s_{1}\right) & \{\mathbf{c}\}\left(r_{1}, s_{2}\right) \\
\{\mathbf{c}\}\left(r_{2}, s_{1}\right) & \{\mathbf{c}\}\left(r_{2}, s_{2}\right)
\end{array}\right]
$$

After solving for the unit response vectors $\{\mathbf{h}\}_{i j}$, the transfer function components $T_{i j}$ are calculated by taking the z-transformation of $\{\mathbf{h}\}_{i j}$, with the argument $z$ set to $e^{i \omega \Delta t}$ :

$$
T_{i j}=\sum_{k=-L}^{L} h_{i j}(k) z^{-k}=\sum_{k=-L}^{L} h_{i j}(k) e^{-i \omega k \Delta t}
$$




\section{E.1 Determining the filter length}

The parameter $L$ is the filter length and is an adjustable parameter. It has an important effect on the accuracy of the system identification. In this section it will be discussed how $L$ can be determined. No strict definitions exist, but guidelines have been defined based on the work of the group of Polifke [30]. The procedure is briefly discussed below.

The first step when determining $L$ is estimating the time delay $\tau$ between the signals $s(t)$ and the responses $r(t)$. The choice for $L$ should at least be larger than this result $[30,32]$ :

$$
L>L_{\text {min }}=\frac{\tau}{\Delta t_{C F D}}
$$

In case the system that has to be identified is a Flame Transfer Function, $\tau$ corresponds to the estimated time lag between velocity fluctuations (signal) and heat release fluctuations (response). For a Flame Transfer Function, it is obvious what the signals and responses are and the causal formulation should be used [30].

On the other hand, in case the system is a purely acoustic component, such as an area discontinuity, the signals and responses are acoustic pressures and velocities, measured on sampling planes located a distance $\Delta x$ from each other. In this case $\tau$ corresponds to the time an acoustic wave needs to travel from the first to the second sampling plane $(\tau=\Delta x / c)$. Because it is hard to distinguish between signals and responses, the non-causal formulation is used.

After the minimum filter length $L_{\text {min }}$ has been determined, the filter length can be optimized by looking at the cross-correlation vector. An example for a purely acoustic MIMO system, with $L_{\text {min }}=30$, is shown in figure E.1(a). This system actually corresponds to the area discontinuity that has been discussed in section D of Chapter 3. For the first choice of $L=L_{m i n}$, the length of the cross correlation vector as defined in equation E.6 can be calculated. It can be seen that all components $c_{i j}$ are much larger than 0 , which indicates that the signals and responses are correlated. The correlation vector is not completely covered by the region of $[-L, L]$, which indicates that the choice for $L$ is too small.

The better choice can be seen in figure E.1(b). It can be seen that all correlation vectors have its maximum in the interval $[-L / 2, L / 2]$. This is a recommendation done by Föller [30] and is based on experience. For values of the index $i$ close to $L$, the signals and responses are not completely uncorrelated, but the correlation is much smaller than the maximum correlation of the main peak in the figures.

The maximum value for $L$ that can be used is not unlimited. If $L$ is chosen too large, the error when inverting matrix equation E.4 will increase, which reduces the identification quality as a consequence. The filter length $L$ should be larger than $L_{\text {min }}$ but still be of the same order of magnitude [64].

Because the choice of $L$ is a bit arbitrary, a sensitivity analysis on the results is recommended when applying the Wiener Hopf Transformation. 

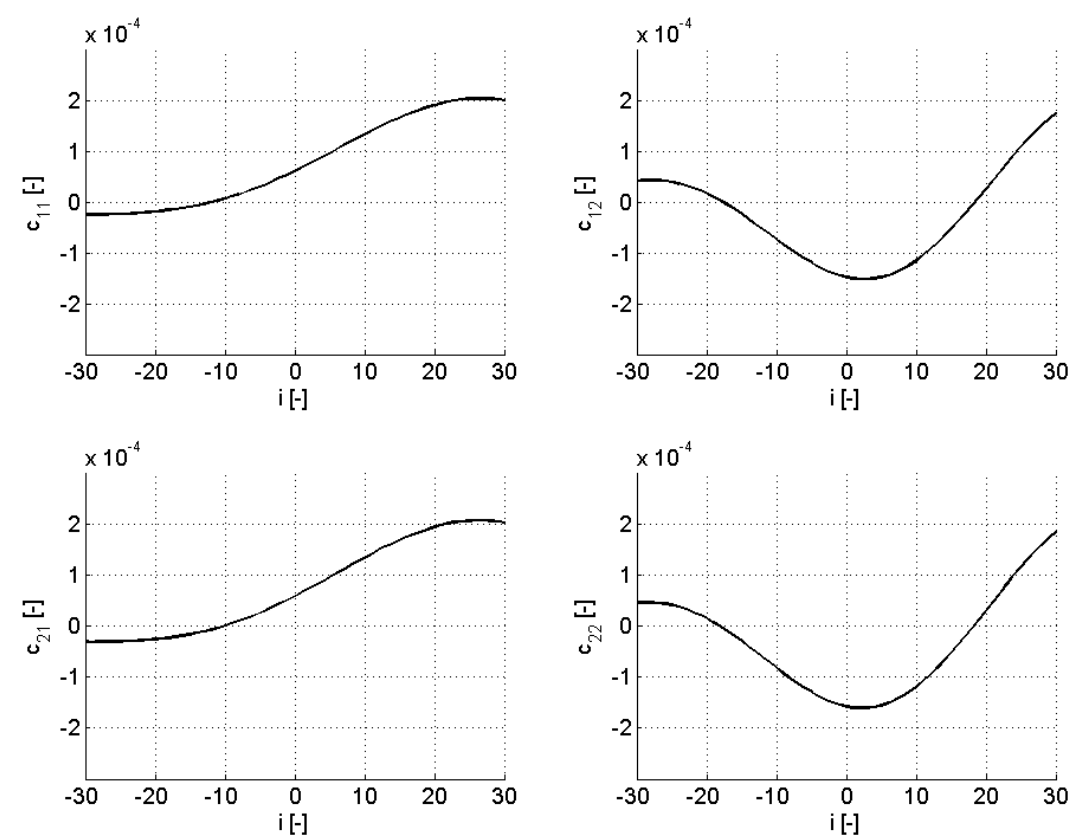

(a) $L=L_{\min }=30$.
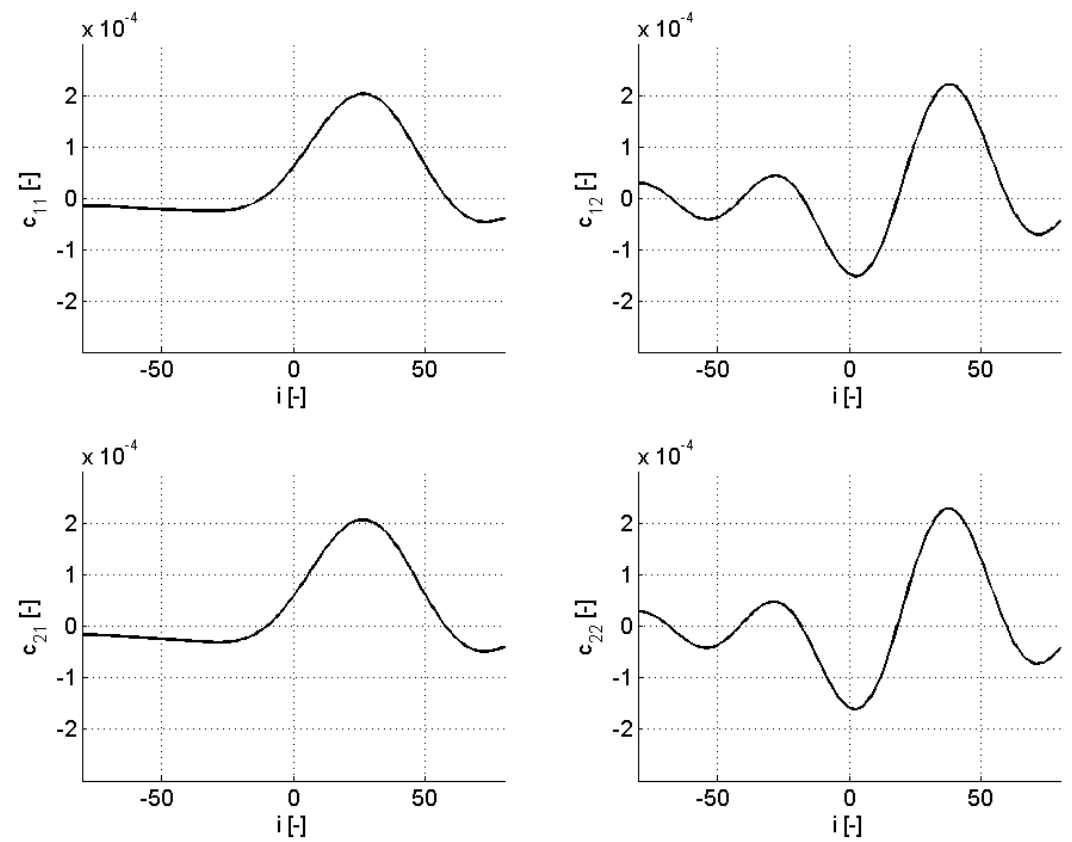

(b) $L=2.67 \cdot L_{\min }=80$.

Figure E.1: Cross correlation vectors for (a) a too small value of $L\left(L=L_{\min }\right)$ and (b) a well chosen value $\left(L=2.67 \cdot L_{\min }=80\right)$ 


\section{F Comparison AVBP and OpenFOAM results}

The swirl stabilized burner, discussed in Chapter 4, has also been numerically studied by CERFACS using their AVBP code. In this appendix the time averaged and root mean square fields for the perfectly premixed case are compared. A comparison of the Flame Transfer Function is performed as well.

\section{F.1 Comparison CFD Setup}

In table F.1 the setup of both the AVBP and OpenFOAM have been compared. A Large Eddy Simulation has been performed with both codes, but the solution algorithm is quite different. AVBP is a fully explicit code, whereas OpenFOAM uses the PISO algorithm. Furthermore quite different computational grids and combustion models have been used. More information on the AVBP setup can be found in Hermeth et al. [39].

\section{F.2 Steady flow field results}

In figure F.1 a contour plot of the mean axial velocity and the mean temperature can be seen. The results obtained using AVBP and OpenFOAM look almost identical. Profiles of the temperature and velocity were extracted at the locations indicated in the figure $(x=0.01 ; 0.02 ; 0: 03 ; 0.04 ; 0.05$ and $0.1 \mathrm{~m}$ from the combustion chamber entrance).

The time averaged temperature profiles are compared in figure F.2. AVBP and OpenFOAM show good overall agreement, although a different approach is used to 


\begin{tabular}{lll}
\hline & AVBP & OpenFOAM \\
\hline $\begin{array}{l}\text { Simulation } \\
\text { type }\end{array}$ & Large Eddy Simulation & Large Eddy Simulation \\
\hline $\begin{array}{l}\text { Solution algo- } \\
\text { rithm }\end{array}$ & Fully compressible explicit & $\begin{array}{l}\text { Fully compressible semi } \\
\text { implicit (PISO) }\end{array}$ \\
\hline $\begin{array}{l}\text { Approxima- } \\
\text { tion }\end{array}$ & Cell-Vertex & Cell centered \\
\hline $\begin{array}{l}\text { Discretization } \\
\text { scheme }\end{array}$ & $\begin{array}{l}2^{\text {nd }} \text { order time and space (Lax- } \\
\text { Wendroff) }\end{array}$ & $\begin{array}{l}2^{\text {nd }} \text { order time and space } \\
\text { (Crank-Nicolson) }\end{array}$ \\
\hline $\begin{array}{l}\text { Grid } \\
\text { Turbulence } \\
\text { model }\end{array}$ & $\begin{array}{l}\text { Unstructured, 7.7M tetrahedral } \\
\text { cells }\end{array}$ & $\begin{array}{l}\text { Unstructured, 3.7M hexa- } \\
\text { hedral cells }\end{array}$ \\
\hline $\begin{array}{l}\text { Combustion } \\
\text { model }\end{array}$ & $\begin{array}{l}\text { Dynamic thickened flame } \\
\text { model with 2 step chemistry } \\
\text { for Methane/Air combustion [15] }\end{array}$ & $\begin{array}{l}\text { Flame Surface Density, } \\
\text { turbulent flame speed } \\
\text { based Bradley [10] }\end{array}$ \\
\hline $\begin{array}{l}\text { Boundary } \\
\text { condition } \\
\text { enthalpy } \\
\text { equation }\end{array}$ & Constant wall heat flux & $\begin{array}{l}\text { Constant wall tempera- } \\
\text { ture }\end{array}$ \\
\hline
\end{tabular}

Table F.1: Comparison computational setup.

model the boundary condition of the enthalpy equation: iso-thermal wall conditions in OpenFOAM against a heat flux through the wall with AVBP. These differences partly explain the different temperatures directly at the wall.

The mean axial, radial and tangential velocity are shown in figure F.3, F.4 and F.5. Again the results obtained with AVBP and OpenFOAM are very similar. PIV measurement data was available for the axial and radial components of the velocity. Compared to PIV data, the inner recirculation zone is well represented by both codes, as well as the location and magnitude of the peak velocity. Both codes over predicted the outer recirculation zone.

Root mean square values of the temperature, velocity and pressure are presented in figures F.6 to F.10. In contrast to the results for the mean flow fields, significant discrepancies can be observed here. Except for the rms-axial velocity (figure F.7), which looks very similar for both codes, AVBP generally predicts much larger rms values. However, the location of minima and maximum corresponds well.

The over prediction of the radial and tangential rms velocity and rms temperature can be explained by looking at the rms pressure (figure F.10). Maximum rmsvalues of about $200 \mathrm{~Pa}$ were found in the OpenFOAM simulations, which was more than 5000 Pa in AVBP (about a factor 25 larger). In AVBP a high frequency azimuthal acoustic mode was excited, which resulted in these high pressure fluctuations. Due 


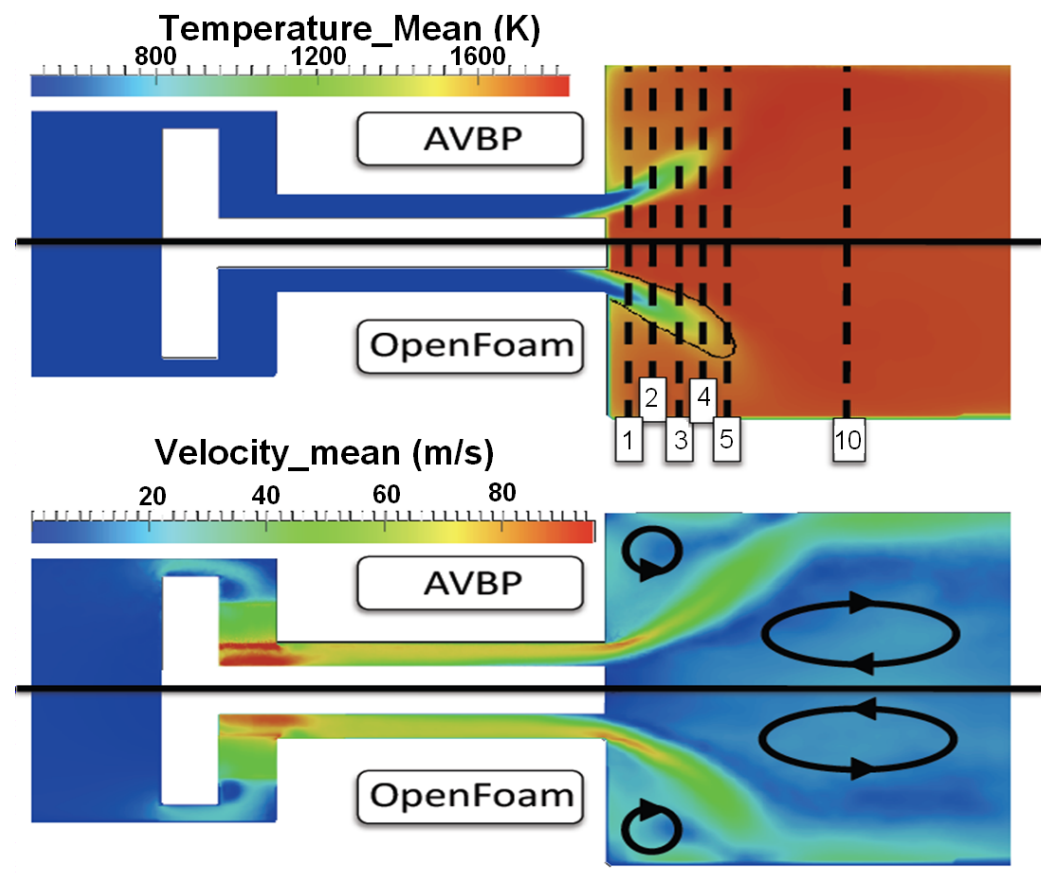

Figure F.1: Steady flow field results for the perfectly premixed case: Comparison of results obtained with OpenFOAM and AVBP. Top plot shows the mean temperature, bottom plot the mean velocity. Both quantities have been averaged over $40 \mathrm{~ms}$.

to the coupling between velocity, temperature and pressure, this also explains why the rms values of the other fields were higher.

\section{F.3 Flame Transfer Function}

Hermeth et al. [39] also determined the Flame Transfer Function with AVBP. In the AVBP simulations, the velocity amplitude in the annular duct between swirler and flame was $u^{\prime} / u_{0}=0.1$. Similar to the OpenFOAM approach, the FTF was determined using single frequency forcing. In figure F.11, the FTF's from OpenFOAM and AVBP are compared to each other and the $O H^{*}$ measurements from the TU Berlin. AVBP data was available for the frequencies $\mathrm{f}=115 \mathrm{~Hz}, 258 \mathrm{~Hz}$ and $323 \mathrm{~Hz}$. The OpenFOAM data shown in figure F.11 is identical to the data shown in figure 4.7 of Chapter 4, but without the data for larger excitation amplitudes.

All simulation results agree well to each other. Compared to measurements, LES under predicts the gain as well as the phase of the FTF for the frequency of $115 \mathrm{~Hz}$. Nevertheless the gain overshoot at this frequency is captured. Saturation of the gain for higher frequencies is also captured with both codes for $258 \mathrm{~Hz}$ and $323 \mathrm{~Hz}$. Over prediction for the phase can be observed from both codes at the higher frequencies. OpenFOAM and AVBP give similar results for all the considered frequencies comforting the LES modeling strategy for such problems. 

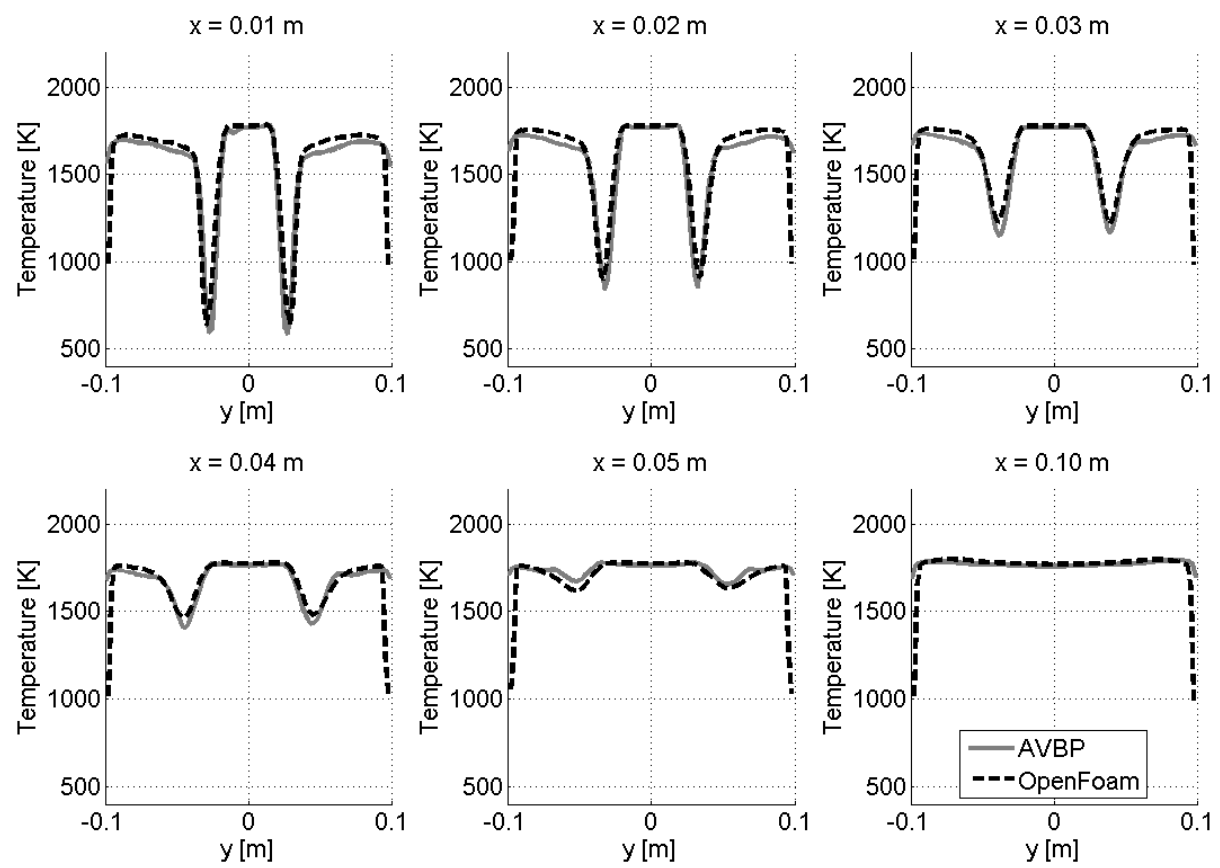

Figure F.2: Mean temperature.
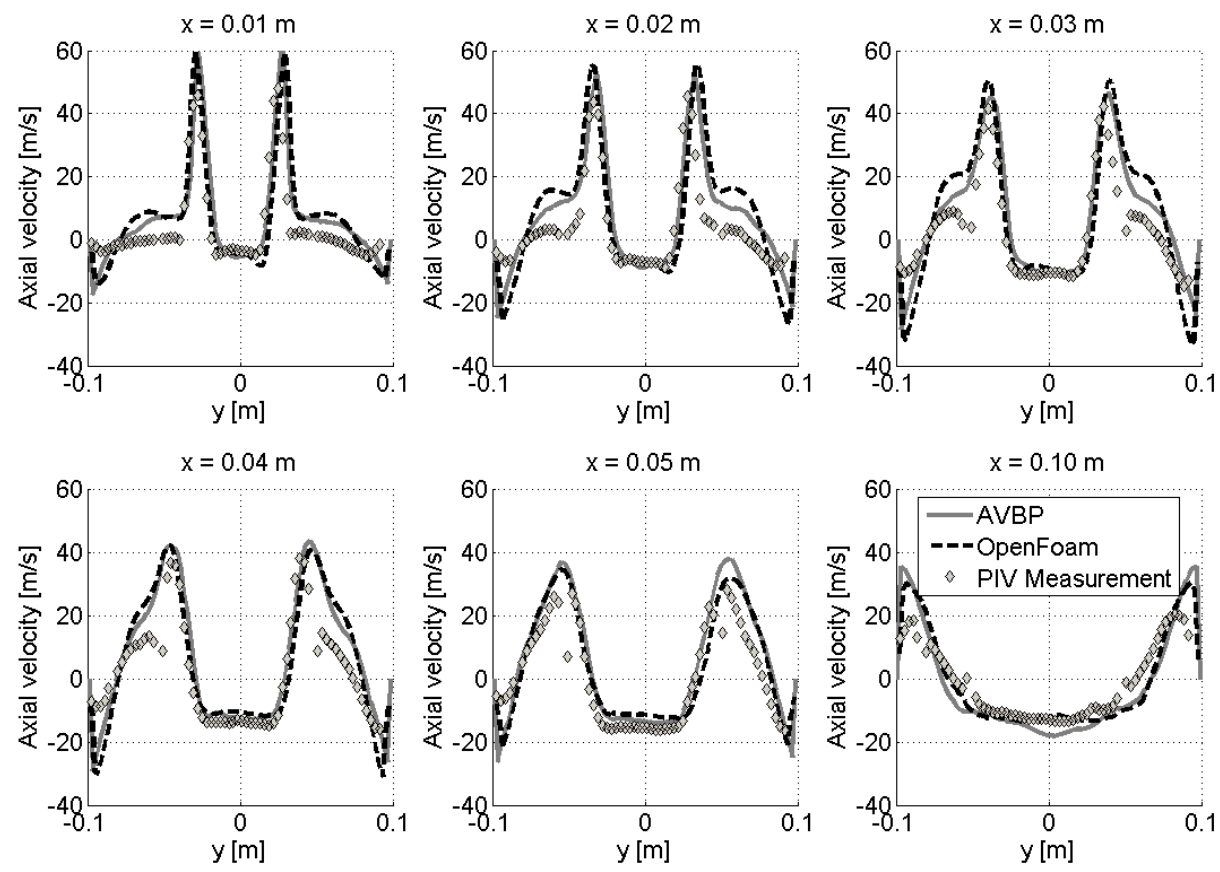

Figure F.3: Mean axial velocity. 

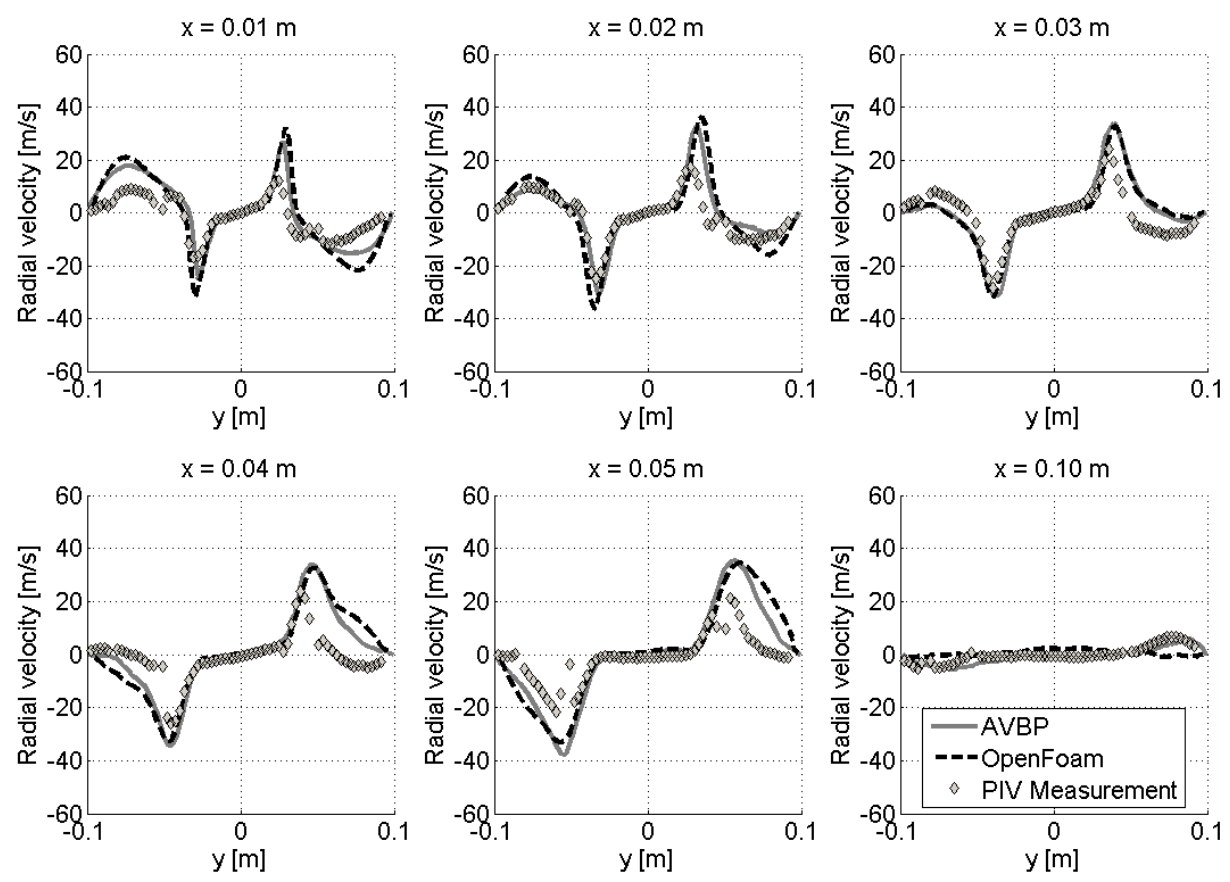

Figure F.4: Mean radial velocity.
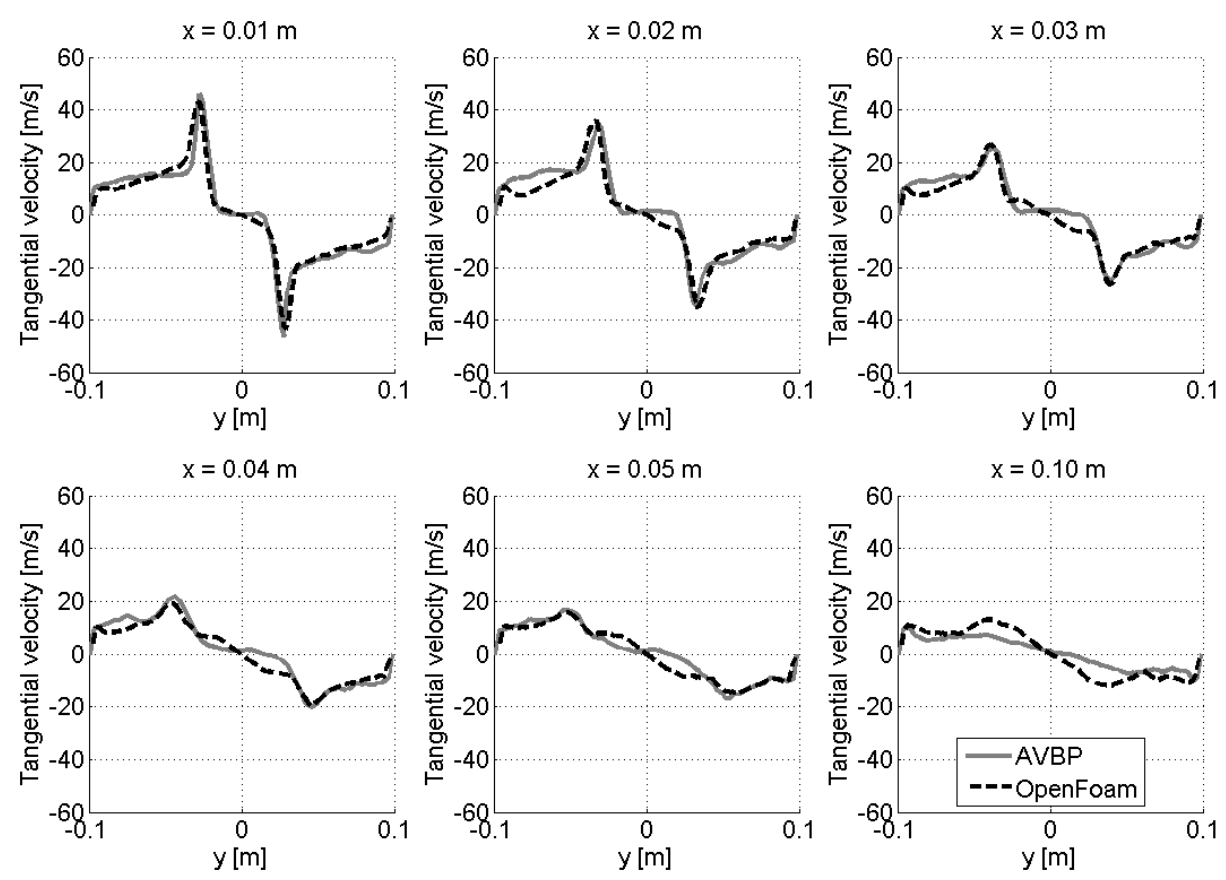

Figure F.5: Mean tangential velocity. 

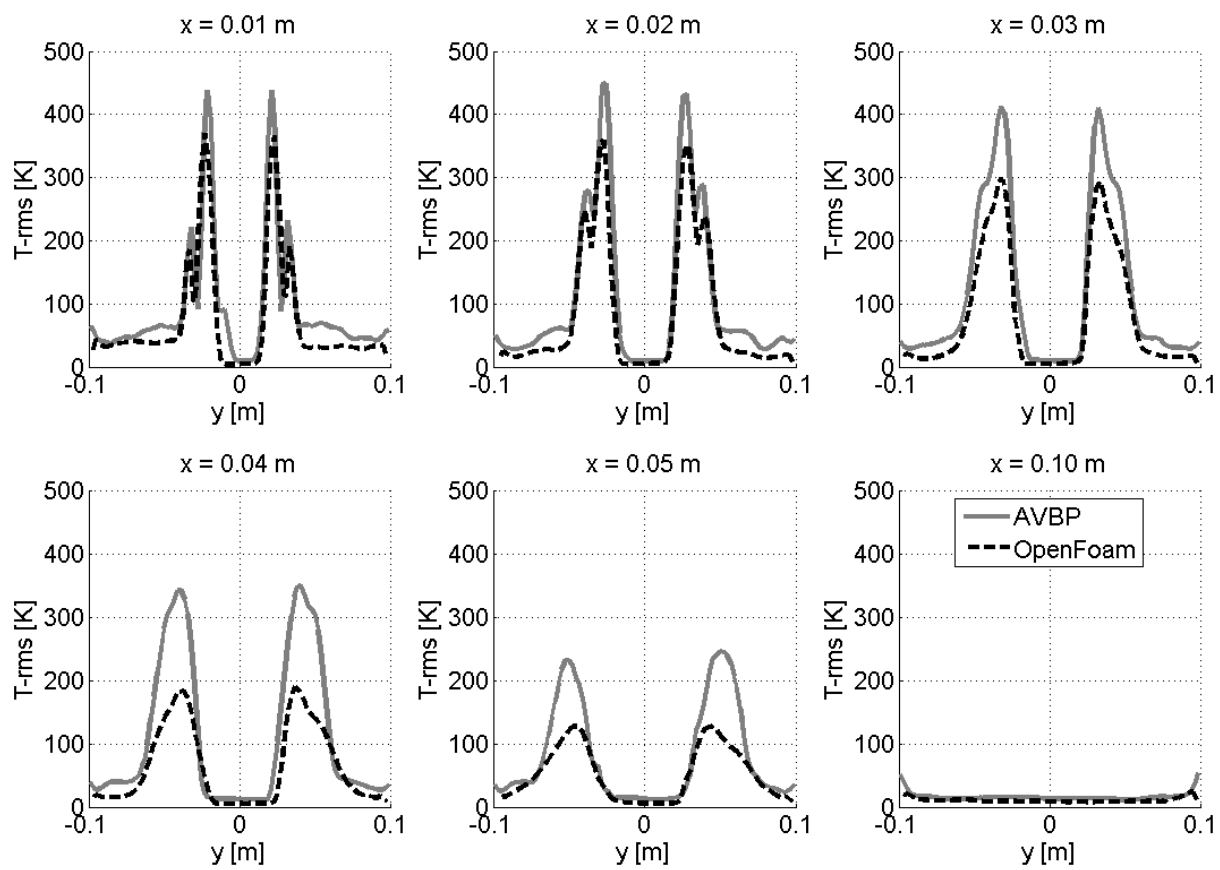

Figure F.6: Root mean square temperature.
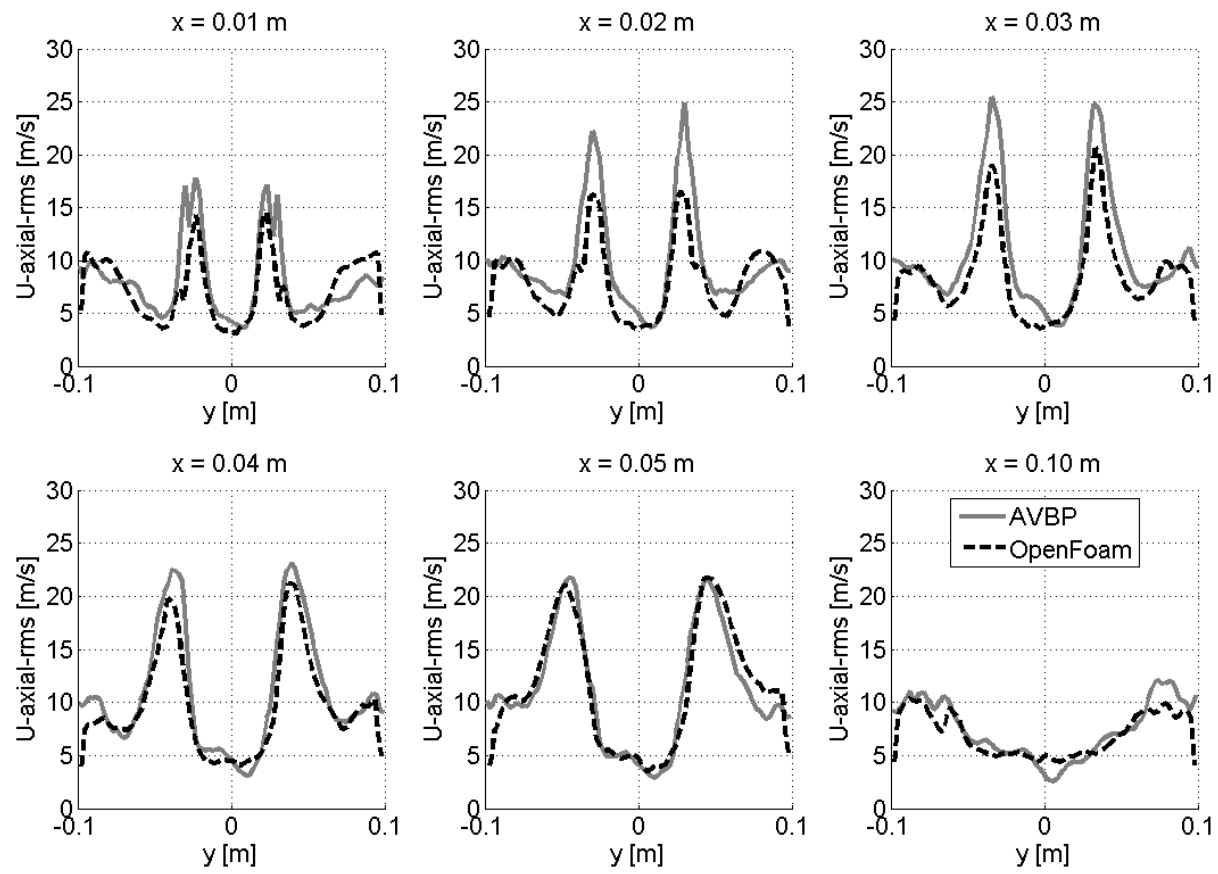

Figure F.7: Root mean square axial velocity. 

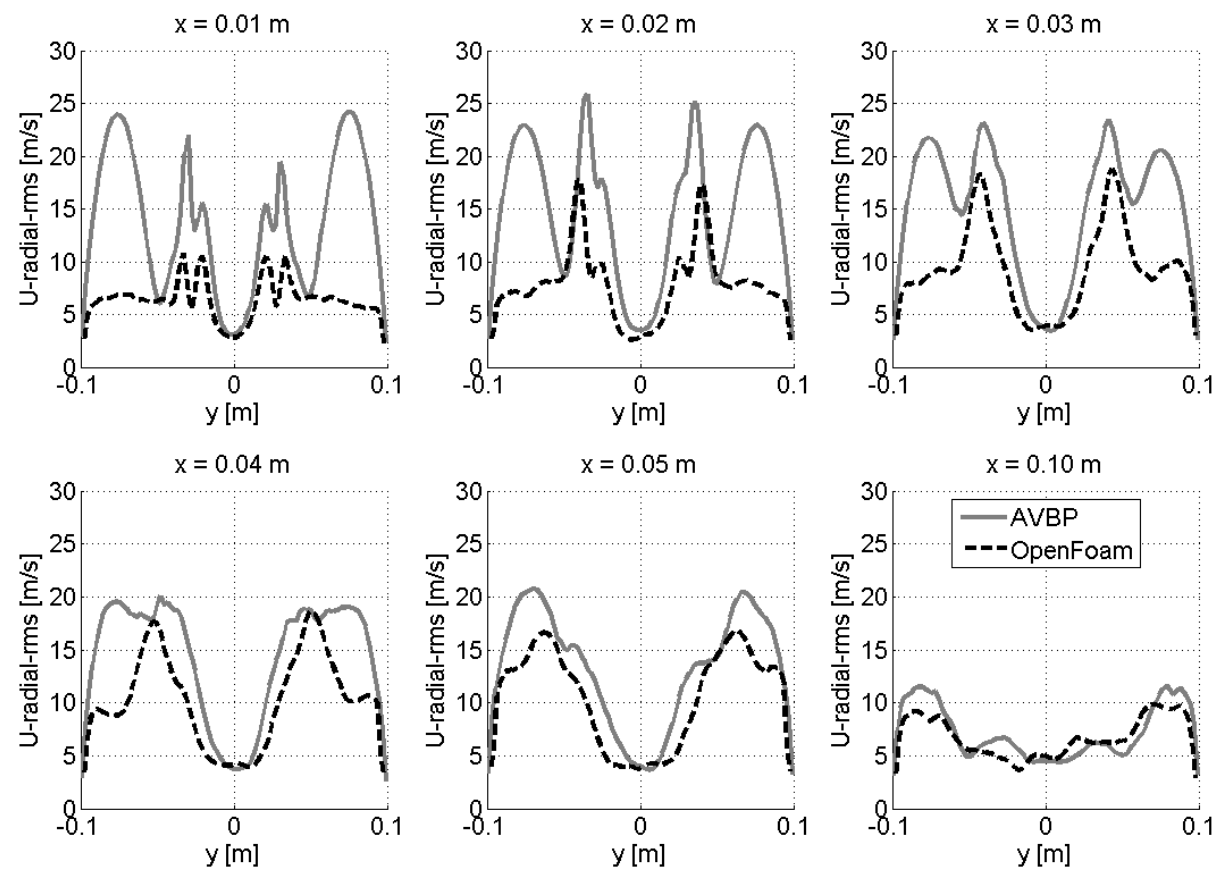

Figure F.8: Root mean square radial velocity.
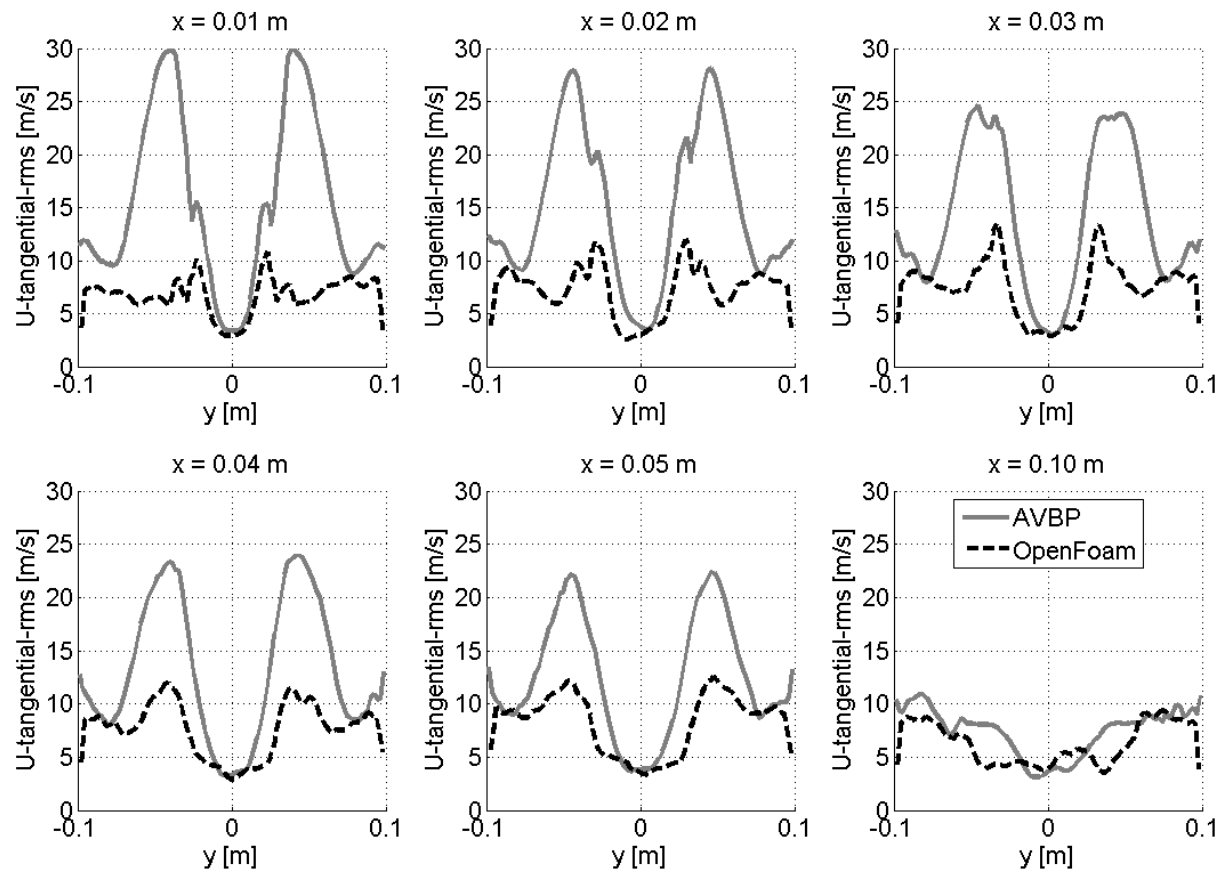

Figure F.9: Root mean square tangential velocity. 

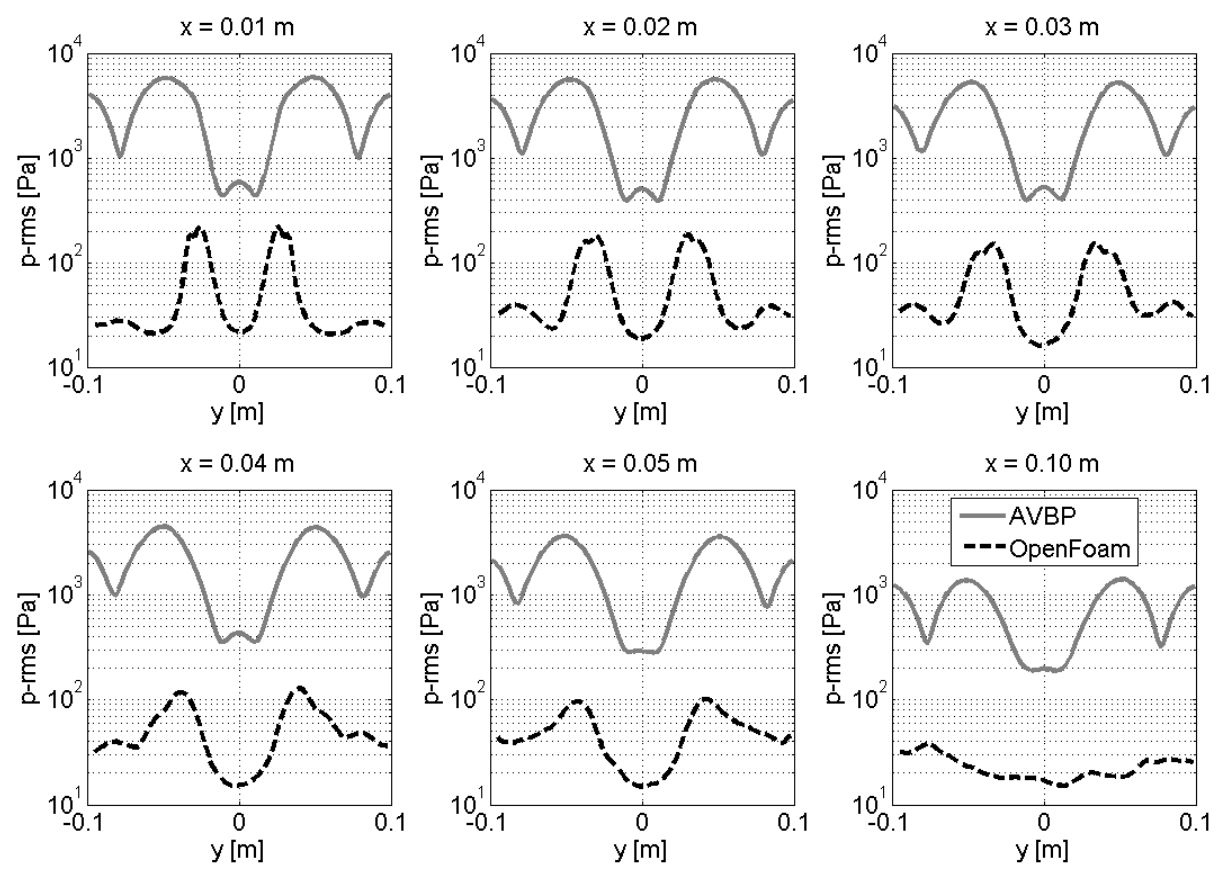

Figure F.10: Root mean square pressure. 


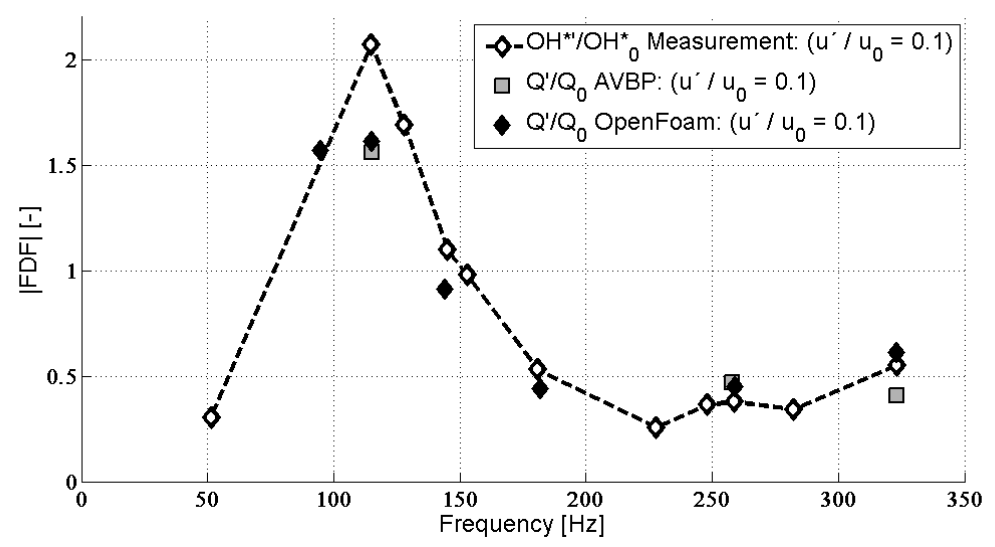

(a) Magnitude.

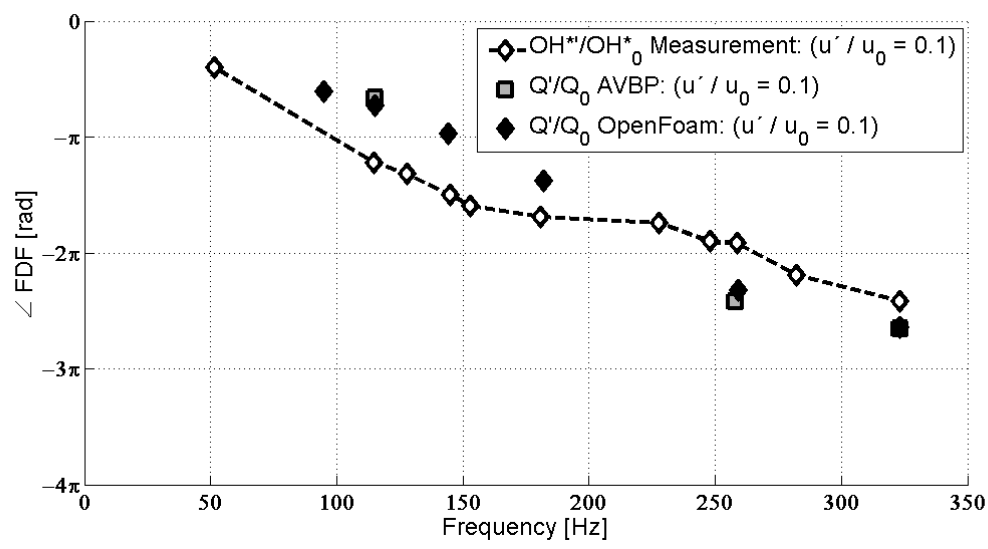

(b) Phase.

Figure F.11: Comparison of measured and simulated Flame Transfer Function obtained with AVBP and OpenFOAM. 


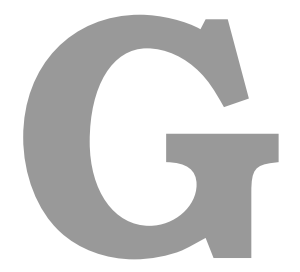

\section{High amplitude multi-frequency excitation}

The simulation of the FDF using single harmonic frequency excitation is a time consuming process. Both for the perfectly and partially premixed case of the FVV burner (Chapter 4), 10 different simulations have been performed (see table 4.4 and table 4.5) and not even the complete FDF was determined.

For the linear Flame Transfer Function, methods using broadband excitation have previously been developed which capture the entire frequency range in only one simulation. Tay et al [12] determined their Flame Transfer Function using the Wiener Hopf transformation (more information about the Wiener Hopf transformation is given in Appendix E). However this approach is not expected to work well for large amplitude excitation.

Therefore a superposition of a small number of harmonic frequencies is applied in this section. A simulation with an excitation signal consisting of $115 \mathrm{~Hz}$ and 258 $\mathrm{Hz}$ frequency components has been performed and compared against single harmonic frequency simulations. In figure G.1 the results of these simulations are compared. In figure G.1(a) time series of the single frequency simulation with $f=115$ $\mathrm{Hz}$ and $u^{\prime} / u_{0}=0.7$ are shown. The excitation signal of the multi-frequency simulation (see figure G.1(b)) was constructed in such a way that the maximum fluctuation was approximately $u^{\prime} / u_{0}=0.7$ as well. However, when looking at the amplitudes of velocity and heat release fluctuations in the frequency domain, see figure G.1(c) and G.1(d), it can be seen that for the simulation with single frequency excitation a peak at $u^{\prime} / u_{0}=0.7$ is found, whereas for the multi-frequency simulation two peaks at approximately $u^{\prime} / u_{0}=0.4$ are found.

Although the combined excitation amplitude in the time domain was about $u^{\prime} / u_{0}=$ 0.7 , this means that the FDF obtained with the multi-frequency simulation should 
not be compared against single frequency simulations with $u^{\prime} / u_{0}=0.7$, but against simulations with $u^{\prime} / u_{0}=0.4$. In figure G.1(e) and G.1(f) the magnitude and phase of the resulting FDF's are compared. The FDF of the multi-frequency simulation was determined in the same way as the FDF of the single frequency simulations (using Discrete Fourier Transformation at the individual frequency components). It can be seen that for the multi-frequency simulation, the magnitude of the FDF at $115 \mathrm{~Hz}$ is significantly lower than the magnitude of the single frequency simulation with $u^{\prime} / u_{0}=0.4$. On the other hand, it compares much better with the simulation with $u^{\prime} / u_{0}=0.7$. This indicates that the nonlinearly in the heat release response at 115 $\mathrm{Hz}$ is enhanced by the additional $258 \mathrm{~Hz}$ component.

Looking at the phase plot, it can also be seen that both frequency components influence each other. In the multi-frequency simulation, the phase at $115 \mathrm{~Hz}$ is slightly lower than the phase from the single frequency simulations, whereas it is higher at $258 \mathrm{~Hz}$.

From these results it should be concluded that the FDF can not be determined using multi-frequency excitation, at least not using the methods used above. It may be possible that other more sophisticated methods may offer a solution here. However, high amplitude multi-frequency excitation was not further investigated within this thesis. 


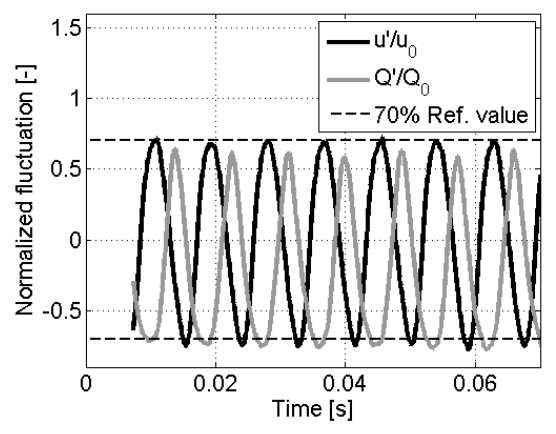

(a) Time series of velocity and heat release fluctuations for mono-frequency simulation with $f=$ $115 \mathrm{~Hz}$ and $u^{\prime} / u_{0}=0.7$.

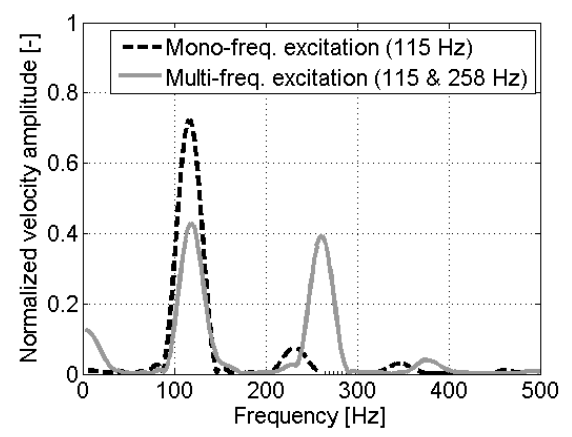

(c) Normalized velocity amplitude of the individual frequency components.

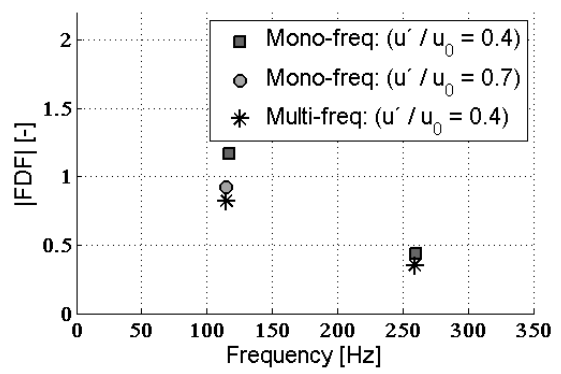

(e) Flame Describing Function at 115 and 258 $\mathrm{Hz}$ - magnitude. The data points for monofrequency excitation correspond to the data points in figure $4.7(\mathrm{a})$.

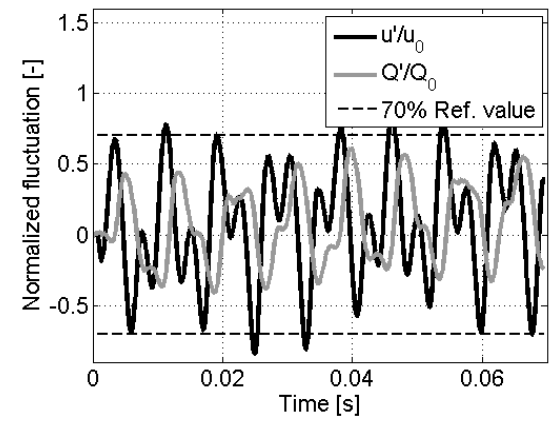

(b) Time series of velocity and heat release fluctuations for multi-frequency simulation with 2 overlaid frequencies: 115 and $258 \mathrm{~Hz}$. The combined excitation level is approximately $u^{\prime} / u_{0}=$ 0.7 .

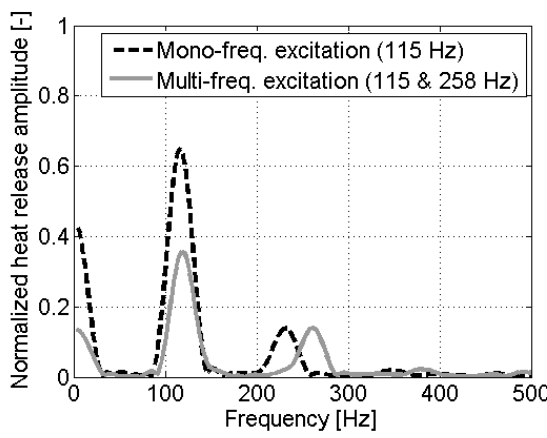

(d) Normalized heat release amplitude of the individual frequency components.

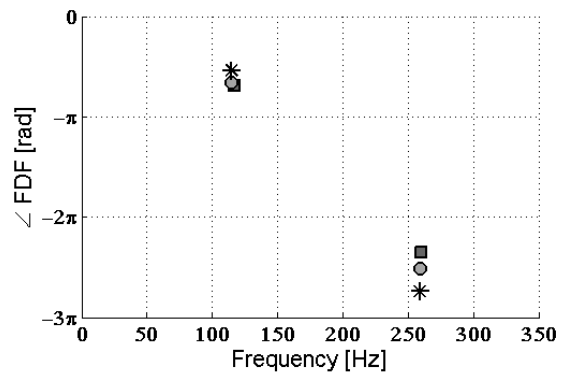

(f) Flame Describing Function at 115 and $258 \mathrm{~Hz}$ - phase. The data points for mono-frequency excitation correspond to the data points in figure 4.7(b).

Figure G.1: Flame Describing Function with high amplitude multi-frequency excitation (perfectly premixed case). 


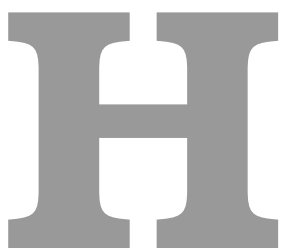

\section{TMA model of the swirl stabilized combustor}

\section{Model}

As mentioned in section Chapter 4, section 4.7.1, a TMA model has been set up, in order to determine the boundary conditions for the GIM model. The TMA model is shown in figure H.1. Whereas the GIM model only contains the flame tube (annular duct) and the combustion chamber, the TMA model also contains the swirler itself and the geometry upstream of the swirl generator.

An important part of the model is the "swirler vanes"-section with the diffuser elements. Both the cross-sectional area and the mean pressure vary linearly across these diffuser elements. The network consists of two branches, which model the two different kinds of passages inside the swirler (see figure 4.16).

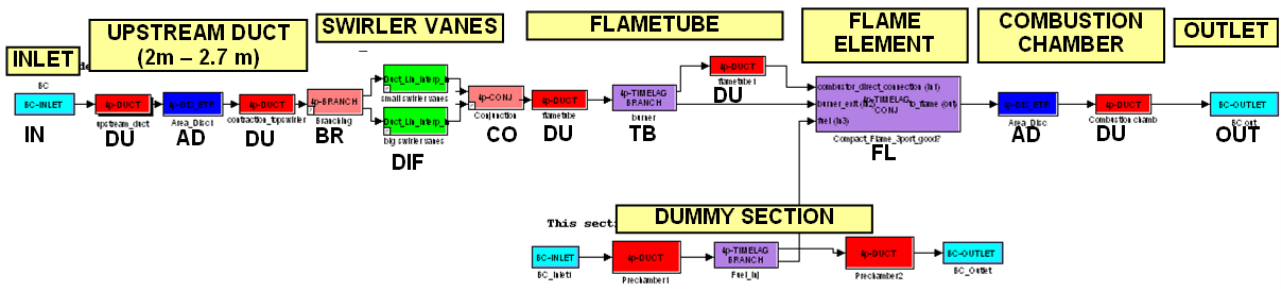

Figure H.1: TMA network model of the rig. IN: Inlet. DU: Duct. AD: Area discontinuity. BR: Branching element. DIF: Diffuser element. CO: Conjunction element. TB: Timelag branch. FL: Flame element. OUT: Outlet.

The model also includes a "dummy section". The flame element is a MISO model and requires 3 inputs (see Appendix A). The dummy section consists of elements 
with extremely large damping and a reflection coefficient of $R=0$ at the outlet. Therefore this section does not contribute to heat release fluctuations. The dummy section does not have a purpose other than closing the network.

\section{Mode shapes}

Using this TMA model, the acoustic mode shapes were calculated. These can be seen in figure H.2. The swirler is located at $x=-0.17 \mathrm{~m}$. The combustion chamber starts at $x=0 \mathrm{~m}$. The mode shapes to the left of the figure (1/2L and $1 \mathrm{~L}$ Upstream mode), have large pressure and velocity amplitudes upstream of the swirler $(x<-0.17 \mathrm{~m})$ and negligible amplitudes in the combustion chamber $(x>0 \mathrm{~m})$. For the mode shapes at the right side of the figure ( $1 / 4 \mathrm{~L}$ and $3 / 4 \mathrm{~L}$ Combustor mode), the opposite is true. This means that the swirler almost decouples the acoustics upstream and downstream of the swirler.
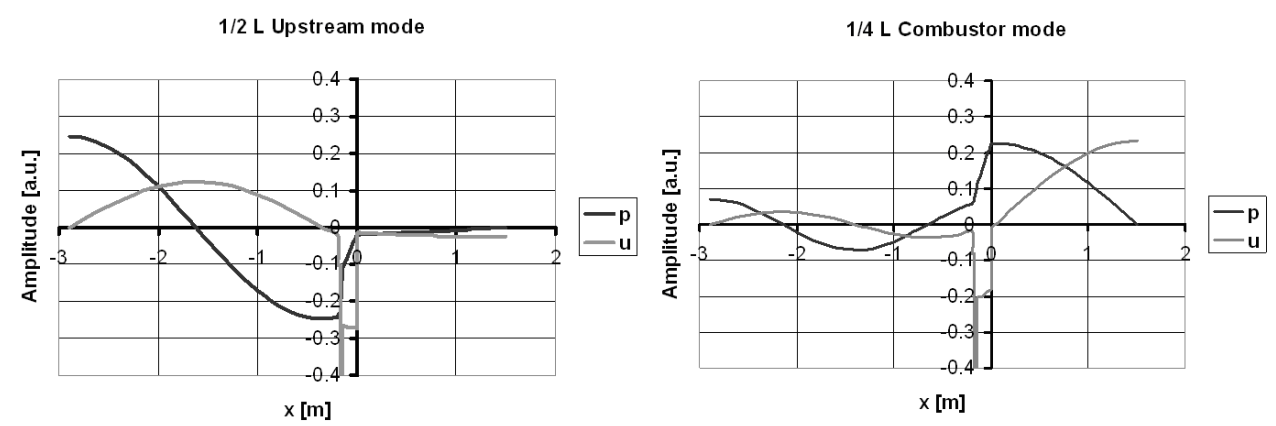

1 L Upstream mode

3/4 L Combustor mode
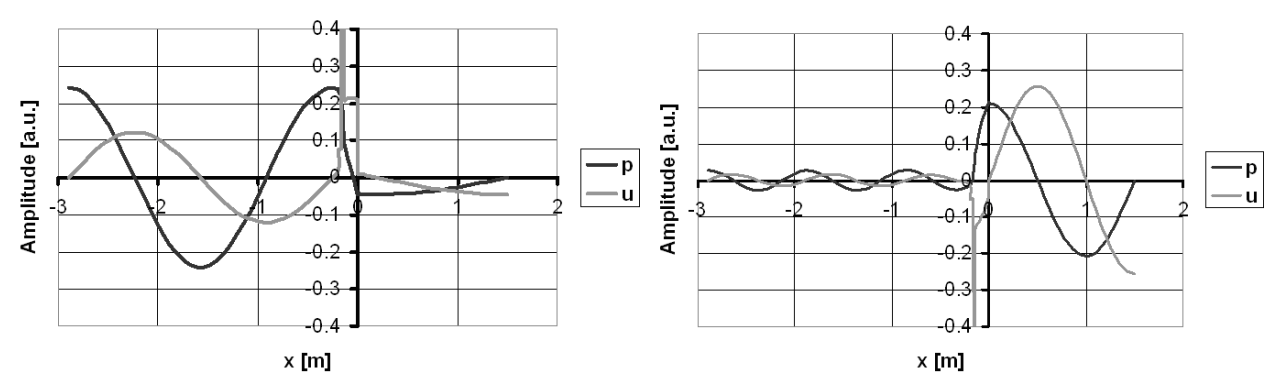

Figure H.2: Mode shapes calculated by TMA. The swirler is located at $x=-0.17 \mathrm{~m}$. The combustion chamber starts at $x=0 \mathrm{~m}$.

The weak coupling between upstream and downstream acoustics is also confirmed in table H.1. The length of the upstream duct could be varied between 2.0 and 2.7 meters (see section 4.2). The table shows that only the frequencies of the $1 / 2 \mathrm{~L}$ and $1 \mathrm{~L}$ upstream mode are sensitive on this length. Furthermore it is interesting to note that it is quite good possible to analytically estimate the eigen frequencies in the combustion chamber. 


\begin{tabular}{l|cc|cc} 
& $\mathbf{L}_{\text {upstr }}:$ & $\mathbf{2 . 0} \mathbf{~ m}$ & $\mathbf{L}_{\text {upstr }}:$ & $\mathbf{2 . 7} \mathbf{~ m}$ \\
\hline Mode & TMA & $\begin{array}{c}\text { Analytical } \\
(f=n \cdot c / L)\end{array}$ & TMA & $\begin{array}{c}\text { Analytical } \\
(f=n \cdot c / L)\end{array}$ \\
\hline 1/4L CB & $149 \mathrm{~Hz}$ & $146 \mathrm{~Hz}$ & $148 \mathrm{~Hz}$ & $146 \mathrm{~Hz}$ \\
3/4L CB & $435 \mathrm{~Hz}$ & $437 \mathrm{~Hz}$ & $436 \mathrm{~Hz}$ & $437 \mathrm{~Hz}$ \\
1/2L upstr. & $117 \mathrm{~Hz}$ & $112 \mathrm{~Hz}$ & $87 \mathrm{~Hz}$ & $83 \mathrm{~Hz}$ \\
1L upstr. & $226 \mathrm{~Hz}$ & $224 \mathrm{~Hz}$ & $169 \mathrm{~Hz}$ & $166 \mathrm{~Hz}$ \\
\hline
\end{tabular}

Table H.1: Eigen frequencies of the burner. The analytical calculation is based on the adiabatic flame temperature $T_{a d}$. The parameter $n$ is the mode number.

\section{Reflection coefficient calculation}

The GIM model in Chapter 4 starts downstream of the swirler. This modeling approach is assumed to be valid, because of the weak coupling between upstream and downstream acoustic modes. Nonetheless, the losses at the swirler need to be known, since these can not be neglected. Therefore the reflection coefficient at the start of the GIM model was determined from TMA. Since the TMA model directly solves the normalized amplitudes $\hat{p}$ and $\hat{u}$, the impedance and thus the reflection coefficient could be calculated. The normalized amplitudes $\hat{p}$ and $\hat{u}$ are extracted directly downstream of the swirler, see figure H.3.

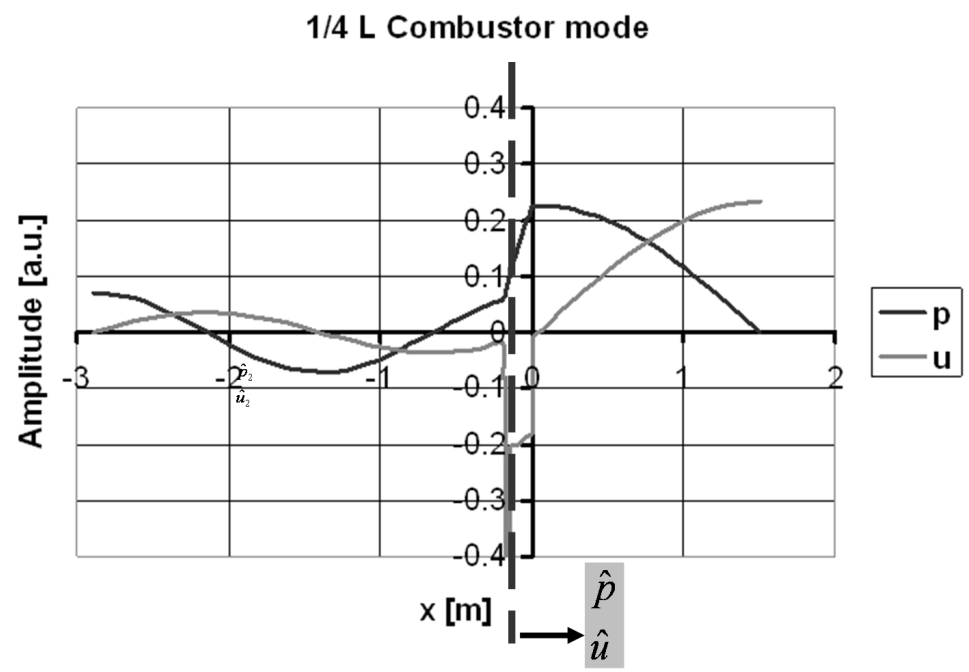

Figure H.3: Extraction of pressure and velocity amplitudes.

The impedance and reflection coefficient are finally calculated from:

$$
\begin{aligned}
& Z=\hat{p} / \hat{u} \\
& R=\frac{Z-\rho c}{Z+\rho c}
\end{aligned}
$$




\section{Specification of CPU resources}

Within this thesis, the following computing clusters have been used:

\begin{tabular}{lll}
\hline Cluster name & IBM iDataPlex $^{*}$ & Juropa $^{\dagger}$ \\
\hline Location & $\begin{array}{l}\text { CERFACS, Toulouse, } \\
\text { France }\end{array}$ & $\begin{array}{l}\text { Forschungszentrum Jülich, } \\
\text { Jülich, Germany }\end{array}$ \\
\hline $\begin{array}{l}\text { Configuration } \\
\text { (node) }\end{array}$ & $\begin{array}{l}\text { 2x Intel Nehalem } \\
\text { quad-core } 2.66 \mathrm{GHz}\end{array}$ & $\begin{array}{l}2 \times \text { Intel Nehalem quad-core } \\
2.93 \mathrm{GHz}\end{array}$ \\
\hline Memory (node) & $24 \mathrm{~GB}$ DDR3 $1333 \mathrm{MHz}$ & $24 \mathrm{~GB}$ DDR3 1066 MHz \\
\hline $\begin{array}{l}\text { Total number } \\
\text { of cores }\end{array}$ & 656 (82 nodes) & 17664 (2208 nodes) \\
\hline Interlink & $\begin{array}{l}\text { Infiniband QDR } \\
\text { Non-blocking }\end{array}$ & Infiniband QDR Non-blocking \\
\hline
\end{tabular}

Table I.1: Technical specifications.

Now follows a detailed specification of the resources used for the individual cases considered within this thesis. Table I.2 contains information about the number of cores used for the different configurations and the amount of CPU and Wall clock time required. (Note: $\mathrm{CPU}$ time $=$ Wall clock time $\mathrm{x}$ Number of cores).

\footnotetext{
*http://www.cerfacs.fr/files/cerfacs/computing/iDataPlex.jpg

${ }^{\dagger}$ http://www.fz-juelich.de/ias/jsc/EN/Expertise/Supercomputers/JUROPA/Configuration/ Configuration_node.html
} 


\begin{tabular}{|c|c|c|c|}
\hline Case & $\begin{array}{l}\text { FVV Perfectly } \\
\text { premixed } \\
\text { (Chapter } 4)\end{array}$ & $\begin{array}{l}\text { FVV Partially } \\
\text { premixed } \\
\text { (Chapter 4) }\end{array}$ & $\begin{array}{l}\text { DOC Partially } \\
\text { premixed } \\
\text { (Chapter 5) }\end{array}$ \\
\hline Cluster & IBM iDataPlex & Juropa & Juropa \\
\hline Grid size & $3,750,782$ cells & $3,274,555$ cells & $16,054,962$ cells \\
\hline Number of cores & 64 (8 nodes) & 128 (16 nodes) & 512 (64 nodes) \\
\hline $\begin{array}{l}\text { Number of steady } \\
\text { simulations }\end{array}$ & 1 & 1 & 1 \\
\hline $\begin{array}{l}\text { Number of harmonic } \\
\text { simulations }\end{array}$ & 10 & 9 & 6 \\
\hline Time step & $5 \cdot 10^{-7} \mathrm{~s}$ & $2 \cdot 10^{-7} \mathrm{~s}$ & $2.5 \cdot 10^{-7} \mathrm{~s}$ \\
\hline $\begin{array}{l}\text { Physical time } \\
\text { (1 harmonic } \\
\text { simulation) }\end{array}$ & $80 \mathrm{~ms}$ & $80 \mathrm{~ms}$ & $50 \mathrm{~ms}$ \\
\hline $\begin{array}{l}\text { Wall clock time } \\
\text { (1 harmonic } \\
\text { simulation) }\end{array}$ & $\begin{array}{l}4.9 \cdot 10^{5} \mathrm{~s} \\
(5.7 \text { days })\end{array}$ & $\begin{array}{l}5.5 \cdot 10^{5} \mathrm{~s} \\
(6.4 \text { days) }\end{array}$ & $\begin{array}{l}4.4 \cdot 10^{5} \\
(5.0 \text { days })\end{array}$ \\
\hline $\begin{array}{l}\text { CPU time } \\
\text { (1 harmonic } \\
\text { simulation) }\end{array}$ & $\begin{array}{l}3.15 \cdot 10^{7} \mathrm{~s} \\
\left(7.1 \cdot 10^{3}\right. \\
\text { CPU-hours) }\end{array}$ & $\begin{array}{l}7.08 \cdot 10^{7} \mathrm{~s} \\
\left(19.7 \cdot 10^{3}\right. \\
\text { CPU-hours) }\end{array}$ & $\begin{array}{l}2.21 \cdot 10^{8} s \\
\left(6.1 \cdot 10^{4}\right. \\
\text { CPU-hours) }\end{array}$ \\
\hline $\begin{array}{l}\text { CPU time } \\
\text { (total) }\end{array}$ & $\begin{array}{l} \pm 3.5 \cdot 10^{8} \mathrm{~s} \\
\left( \pm 7.8 \cdot 10^{4}\right. \\
\text { CPU-hours) }\end{array}$ & $\begin{array}{l} \pm 7.08 \cdot 10^{8} \mathrm{~s} \\
\left( \pm 19.7 \cdot 10^{4}\right. \\
\text { CPU-hours })\end{array}$ & $\begin{array}{l} \pm 1.55 \cdot 10^{9} \mathrm{~s} \\
\left( \pm 4.38 \cdot 10^{5}\right. \\
\text { CPU-hours })\end{array}$ \\
\hline
\end{tabular}

Table I.2: Number of cores and required CPU time for the individual cases. 


\section{Nomenclature}

In the nomenclature, all symbols which have been used in this thesis are summarized. They are categorized in Roman symbols, Greek symbols, Accents and Mathematical operators and Abbreviations. Some symbols have been used for different quantities. In this case, the meaning follows from the context.

\begin{tabular}{|c|c|c|}
\hline & Roman symbols & \\
\hline$A$ & Area & {$\left[m^{2}\right]$} \\
\hline$A_{F S D}$ & Constant of the combustion model & {$[-]$} \\
\hline$c$ & Reaction progress variable & {$[-]$} \\
\hline$c$ & Speed of sound & {$[\mathrm{m} / \mathrm{s}]$} \\
\hline$C_{P}$ & Specific heat capacity at constant pressure & {$[\mathrm{J} / \mathrm{kg} / \mathrm{K}]$} \\
\hline$C_{S}$ & Constant of the Smagorinsky model & {$[-]$} \\
\hline$C_{V}$ & Specific heat capacity at constant volume & {$[\mathrm{J} / \mathrm{kg} / \mathrm{K}]$} \\
\hline Co & Courant number & {$[-]$} \\
\hline$D$ & Diameter & {$[m]$} \\
\hline$D_{k}$ & Diffusion coefficient of species $k$ & {$\left[\mathrm{~m}^{2} / \mathrm{s}\right]$} \\
\hline$D_{T}$ & Turbulent diffusion coefficient & {$\left[\mathrm{m}^{2} / \mathrm{s}\right]$} \\
\hline$e$ & Internal energy & {$[\mathrm{J} / \mathrm{kg}]$} \\
\hline$e^{\prime}$ & Local acoustic energy & {$\left[\mathrm{J} / \mathrm{m}^{3}\right]$} \\
\hline$E^{\prime}$ & Volume integrated acoustic energy & {$[J]$} \\
\hline$f$ & Frequency & {$[\mathrm{Hz}]$} \\
\hline$f_{T}$ & Mixture fraction & {$[-]$} \\
\hline$F$ & Flame Transfer Function & {$[-]$} \\
\hline $\mathcal{F}$ & Flame Describing Function & {$[-]$} \\
\hline$F_{n}$ & Source term in the Generalized Instability Model & {$\left[\mathrm{kgm}^{2} / \mathrm{s}^{4}\right]$} \\
\hline$h$ & Enthalpy & {$[\mathrm{J} / \mathrm{kg}]$} \\
\hline$h$ & Source term in the Generalized Instability Model & {$\left[\mathrm{kg} / \mathrm{m}^{3} / \mathrm{s}^{2}\right]$} \\
\hline$h_{k}$ & Specific enthalpy of species $k$ & {$[\mathrm{~J} / \mathrm{kg}]$} \\
\hline h & Unit response vector & [a.u.] \\
\hline$H$ & Window function & {$[-]$} \\
\hline$\widehat{H}$ & Normalized window function & {$[-]$} \\
\hline$H_{f}$ & Specific enthalpy of reaction & {$[\mathrm{J} / \mathrm{kg}$ fuel $]$} \\
\hline$k$ & Acoustic wave number $(=\omega / c)$ & {$[1 / m]$} \\
\hline$k^{ \pm}$ & Acoustic wave number corrected by mean flow & {$[1 / m]$} \\
\hline Ka & Karlovitz number & {$[1 / m]$} \\
\hline I & Identity matrix & {$[-]$} \\
\hline
\end{tabular}


$I_{O H^{*}} \quad$ Intensity of $O H^{*}$ chemiluminescence

[a.u.]

$l_{T} \quad$ Characteristic turbulent length scale

$L \quad$ Length

$L_{1}, L_{5} \quad$ Characteristic acoustic wave (LODI model)

Le Lewis number

$m \quad$ Mass flow

$M a \quad$ Mach number

$M a^{*} \quad$ Model parameter of the combustion model $\left[\mathrm{kg} / \mathrm{m} / \mathrm{s}^{3}\right]$

$n \quad$ Interaction index

$p \quad$ Pressure

$\operatorname{Pr} \quad$ Prandtl number

$q \quad$ Heat release density

$Q \quad$ Volume integrated heat release

$R \quad$ Reflection coefficient

$R \quad$ Specific gas constant of air

$R_{u u} \quad$ Auto-correlation vector of signal $u$

$R_{u Q} \quad$ Cross-correlation vector between signals $u$ and $Q$

Re Reynolds number

$S \quad$ Swirl number

Sc Schmidt number

$S_{L} \quad$ Laminar flame speed

$S_{L}^{0} \quad$ Unstrained laminar flame speed

$S_{T} \quad$ Turbulent flame speed

S Strain rate tensor

$t \quad$ Time

$T \quad$ Temperature

$T_{a d} \quad$ Adiabatic flame temperature

$T_{u} \quad$ Unburnt temperature

T Reynolds stress tensor

T Transfer matrix (general notation)

$u, \mathbf{u} \quad$ Velocity

$u_{S G S}^{\prime \prime} \quad$ Turbulent subgrid velocity

$V \quad$ Volume

$\dot{V} \quad$ Volume flow

$\dot{w}_{k} \quad$ Mass conversion rate of species $k$

$\dot{w}_{R} \quad$ Fuel conversion rate

$W \quad$ Rate of acoustic energy production

$x, y, z \quad$ Coordinate

$Y_{k} \quad$ Mass fraction of species $k$

$Z \quad$ Acoustic impedance

Ze Zeldovitch number

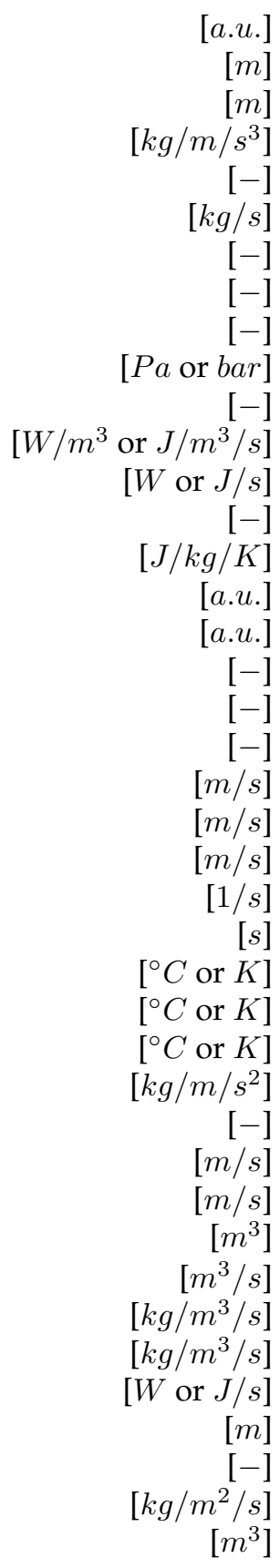

Greek symbols

\begin{tabular}{llr}
\hline$\alpha$ & Thermal diffusivity & {$\left[\mathrm{m}^{2} / \mathrm{s}\right]$} \\
$\beta$ & Dimensionless heat loss parameter & {$[-]$} \\
$\Delta$ & LES filter width & {$[-]$}
\end{tabular}




\begin{tabular}{llr}
$\eta$ & Temporal evolution of acoustic mode & {$[-]$} \\
$\eta_{K o l}$ & Kolmogorov length scale & {$[\mathrm{m}]$} \\
$\gamma$ & Ratio of specific heats $C_{P} / C_{V}$ & {$[-]$} \\
$\Gamma$ & Growth Rate & {$[\mathrm{rad} / \mathrm{s}]$} \\
$\Gamma$ & Auto-correlation matrix & {$[\mathrm{a} . \mathrm{u}]$.} \\
$\zeta$ & Damping factor & {$[-]$} \\
$\kappa$ & Strain rate & {$[1 / \mathrm{m}]$} \\
$\lambda$ & Acoustic wave length $(\mathrm{c} / \mathrm{f})$ & {$[\mathrm{m}]$} \\
$\lambda$ & Thermal conductivity & {$[\mathrm{W} / \mathrm{m} / \mathrm{K}]$} \\
$\mu$ & Dynamic viscosity & {$[\mathrm{kg} / \mathrm{m} / \mathrm{s}]$} \\
$\mu_{T}$ & Turbulent viscosity & {$[\mathrm{kg} / \mathrm{m} / \mathrm{s}]$} \\
$\nu$ & Kinematic viscosity & {$\left[\mathrm{m}^{2} / \mathrm{s}\right]$} \\
$\Xi$ & Ratio strained / unstrained lam. flame speed $\left(S_{L} / S_{L}^{0}\right)$ & {$[-]$} \\
$\rho$ & Density & {$\left[\mathrm{kg} / \mathrm{m}^{3}\right]$} \\
$\rho_{u}$ & Unburnt density & {$\left[\mathrm{kg} / \mathrm{m}^{3}\right]$} \\
$\Sigma$ & Ratio turbulent / laminar flame speed $\left(S_{T} / S_{L}\right)$ & {$[-]$} \\
$\tau$ & Time lag & {$[\mathrm{s}]$} \\
$\tau$ & Time scalar (LES boundary condition) & {$[\mathrm{s}]$} \\
$\overline{\bar{\tau}}$ & Viscous stress tensor & {$[\mathrm{Pa}]$} \\
$\Phi$ & Equivalence ratio & {$[-]$} \\
$\chi$ & Model parameter of the combustion model & {$[-]$} \\
$\Psi$ & Spatial distribution of acoustic mode & {$[-]$} \\
$\omega$ & Frequency & {$[\mathrm{rad} / \mathrm{s}]$} \\
\hline
\end{tabular}

\begin{tabular}{lll}
\hline & Accents and mathematical operators \\
\hline$\hat{f}$ & Fluctuation of quantity $f$ (Frequency domain) & \\
$f^{\prime}$ & Fluctuation of quantity $f$ (Time domain) & \\
$f^{\prime \prime}$ & Subgrid fluctuation of quantity $f$ & \\
$f_{0}$ & Mean value of quantity $f$ & \\
$\bar{f}$ & LES filtered part of quantity $f$ & {$[\mathrm{rad}]$} \\
$\tilde{f}$ & Favre weighted LES filtered part of quantity $f$ & \\
$|f|$ & Absolute value / magnitude of quantity $f$ & \\
$\angle f$ & Phase of quantity $f$ & \\
$f_{\infty}$ & Far stream value of quantity $f$ \\
\hline & Abbreviations \\
\hline
\end{tabular}

CFD Computational Fluid Dynamics

CFL Courant-Freidrichs-Levy number

DNS Direct Numerical Simulation

FDF Flame Describing Function

FFT Fast Fourier Transfer

FSD Flame Surface Density

FTF Flame Transfer Function

GIM Generalized Instability Model

LES Large Eddy Simulation

MISO Multiple Input Multiple Output 
PIV Particle Image Velocimetry

RANS Reynolds Averaged Navier Stokes

SISO Single Input Single Output

SPL Sound Pressure Level 


\section{Publications}

\section{Conference papers}

- H.J. Krediet, J.E. Portillo, W. Krebs and J.B.W. Kok, Prediction of Thermoacoustic Limit Cycles during Premixed Combustion using the Modified Galerkin Approach, $46^{\text {th }}$ AIAA/ASME/SAE/ASEE Joint Propulsion Conference and Exhibit, Nashville, United States of America, 2010. [56]

- H.J. Krediet, C.H. Beck, W. Krebs, S. Schimek and C.O. Paschereit, Identification of the Flame Describing Function of a premixed swirl flame from LES, $7^{\text {th }}$ Mediterranean Combustion Symposium, Chia Laguna, Sardinia, Italy, 2011. [55]

- S. Hermeth, G. Staffelbach, L. Gicquel, T. Poinsot, H.J. Krediet and W. Krebs, LES and Flame Transfer Function measurement in a perfectly premixed swirled burner, INCA Conference, Toulouse, 2011. [39]

- W. Krebs, H.J. Krediet, J.E. Portillo, S. Hermeth, T. Poinsot, S. Schimek and C.O. Paschereit, Comparison of nonlinear to linear thermoacoustic stability analysis of a gas turbine combustion system, ASME Turbo Expo, GT2012-69477, Copenhagen, Denmark, 2012.

- H.J. Krediet, C.H. Beck, W. Krebs and J.B.W. Kok, Saturation mechanism of the heat release response of a premixed swirl flame using LES, $34^{\text {th }}$ International Combustion Symposium, Warsaw, Poland, 2012.

\section{Journal papers}

- H.J. Krediet, C.H. Beck, W. Krebs, S. Schimek, C.O. Paschereit and J.B.W. Kok, Identification of the Flame Describing Function of a premixed swirl flame from LES, Combustion Science and Technology (in preparation).

- H.J. Krediet, W. Krebs, C.H. Beck, J.E. Portillo and J.B.W. Kok, Prediction of limit cycle pressure oscillations in a swirl stabilized combustor using Flame Describing Functions from LES, Journal of Spray and Combustion Dynamics (in preparation).

- H.J. Krediet, C.H. Beck, W. Krebs and J.B.W. Kok, Saturation mechanism of the heat release response of a premixed swirl flame using LES, Proceedings of the Combustion Institute (in preparation). 


\section{Bibliography}

[1] http://www.openfoam.com.

[2] K. Arens. Numerische Berechnung Thermoakustischer Instabilitäten einer 3D Benchmark Brennkammer. PhD thesis, Technische Universität München, Fakultät für Mathematik, 2009.

[3] C.A. Armitage, R. Balachandran, E. Mastorakos, and R.S. Cant. Investigation of the nonlinear response of turbulent premixed flames to imposed inlet velocity oscillations. Combustion and Flame, 146:419-436, 2006.

[4] C.A. Armitage, A.J. Riley, R.S. Cant, A.P. Dowling, and S.R. Stow. Flame transfer function for swirled LPP combustion from experiments and CFD. Proceedings ASME Turbo Expo, Vienna, 2004.

[5] R. Balachandran, B.O. Ayoola, C.F. Kaminski, A.P. Dowling, and E. Mastorakos. Experimental investigation of the nonlinear response of turbulent premixed flames to imposed inlet velocity oscillations. Combustion and Flame, 143:37-55, 2005.

[6] R. Balachandran, A.P. Dowling, and E. Mastorakos. Non-linear response of premixed flames to superimposed harmonic inlet velocity fluctuations of multiple frequencies. $41^{\text {th }}$ AIAA/ASME/SAE/ASEE Joint Propulsion Conference and Exhibit, Tucson.

[7] K. Balasubramanian and R.I. Sujith. Thermoacoustic instability in a Rijke tube: Non-normality and nonlinearity. Physics of Fluids, 20:225-243, 2008.

[8] C. Beck. DOC HP test 2011 Results - dynamics \& interpretation. Siemens internal report, 2011.

[9] V. Belluci, B. Schuermans, D. Novak, P. Flohr, and C.O. Paschereit. Thermoacoustic modeling of a gas turbine combustor equipped with acoustic dampers. Proceedings ASME Turbo Expo, Vienna, GT2004-53977, 2004.

[10] D. Bradley. How fast can we burn? Proceedings of the Combustion Institute, 24:247-262, 1992.

[11] P. Chatterjee, U. Vandsburger, W.R. Saunders, V.K. Khanna, and W.T. Baumann. On the spectral characteristics of a self-excited Rijke tube combustor - numerical simulation and experimental measurements. Journal of Sound and Vibration, 283:573-588, 2005. 
[12] L. Tay Wo Chong, T. Komarek, R. Kaess, S. Föller, and W. Polifke. Identification of Flame Transfer Functions from LES of a premixed swirl burner. Proceedings ASME Turbo Expo, Glasgow, GT2010-22769, 2010.

[13] L. Tay Wo Chong, T. Komarek, M. Zellhuber, J. Lenz, C. C. Hirsch, and W. Polifke. Influence of strain and heat loss on flame stabilization in a non-adiabatic combustor. European combustion meeting, 2009.

[14] T. Clark and D. Bittker. A study of the radiation from laminar and turbulent open propane-air flames as a function of flame area, equivalence ratio, and fuel flow rate. National Advisory Committee for Aeronautics, Lewis Flight Propulsion Laboratory Cleveland, Ohio, 1954.

[15] O. Colin, F. Ducros, D. Veynante, and T. Poinsot. A thickened flame model for large eddy simulations of turbulent premixed combustion. Physics of Fluids, 12(7):1843-1863, 2000.

[16] L. Crocco. Aspects of combustion instability in liquid propellant rocket motors, Part i. Journal of the American Rocket Society, 21:163-178, 1951.

[17] L. Crocco. Aspects of combustion instability in liquid propellant rocket motors, Part ii. Journal of the American Rocket Society, 22:11-26, 1952.

[18] F.E.C. Culick. Unsteady motions in combustion chambers for propulsion systems. AGARDograph, 2006.

[19] F.E.C. Culick and C.C. Jahnke. Application of dynamical systems theory to nonlinear combustion instabilities. Journal of Propulsion and Power, 10(4):508518, 1994.

[20] F.E.C. Culick and V. Yang. Prediction of the Stability of Unsteady Motions in Solid-Propellant Rocket Motors. Progress in Aeronautics and Astronautics, AIAA, 143:719-779, 1992.

[21] F.E.C. Culick and V. Yang. Overview of Combustion Instabilities in LiquidPropellant Rocket Engines. Progress in Aeronautics and Astronautics, AIAA, 169:337, 1995.

[22] M.A.F. Derksen. On the influence of steam on combustion. PhD thesis, University of Twente, 2005.

[23] M. Di Domenico, C.H. Beck, O. Lammel, W. Krebs, and B. Noll. Experimental and numerical investigation of turbulent, lean, high-strained, confined, jet flames. Proceedings 49th AIAA Aerospace Sciences Meeting (Orlando), 2011.

[24] A.P. Dowling. Nonlinear self-excited oscillations of a ducted flame. Journal of Fluid Mechanics, 346:271-290, 1997.

[25] A.P. Dowling. A kinematic model of a ducted flame. Journal of Fluid Mechanics, 394:51-72, 1999. 
[26] A.P. Dowling. Modeling and control of combustion instabilities. Proceedings ASME Turbo Expo, Reno-Tahoe, Nevada, 2005.

[27] A.P. Dowling and A.S. Morgans. Feedback control of combustion instabilities. Annu. Rev. Fluid. Mech., 37:151-182, 2005.

[28] A.P. Dowling and S.R. Stow. Acoustic analysis of gas turbine combustors. Journal of Propulsion and Power, 19(5):751-763, 2003.

[29] A.P. Dowling and S.R. Stow. A time-domain network model for nonlinear thermoacoustic oscillations. Proceedings ASME Turbo Expo, Berlin, 2008.

[30] S. Föller, A.M.G. Gentemann, T. Komarek, and W. Polifke. User Manual WHI Post Processor, a Correlation Based Signal Analysis Tool for Single-Input, Single-Output (SISO) and Multiple-Input, Multiple-Output (MIMO) Systems.

[31] F. Galeazzo, G. Donnert, P. Habisreuther, N. Zarzalis, R.J. Valdes, and W. Krebs. Measurement and simulation of turbulent mixing in a jet in crossflow. ASME Turbo Expo, GT2010-22709, 2010.

[32] A. Gentemann, A. Fischer, S. Evesque, and W. Polifke. Acoustic Transfer Matrix Reconstruction and Analysis for Ducts with a sudden change of area. 9th AIAA/CEAS Aeroacoustics Conference and Exhibit, 2003.

[33] K.J. George and R.I. Sujith. On Chu"s disturbance energy. Journal of Sound and Vibration, 330(22):5280-5291, 2011.

[34] S. Gövert. Analysis of unsteady flow phenomena in gas turbine combustion systems based on large eddy simulations. Master's thesis, Otto von Guericke Universität Magdeburg, Fakultät für Maschinenbau, 2011.

[35] O.L. Gülder. Turbulent premixed flame propagation models for different combustion regimes. Proceedings of the Combustion Institute, pages 743-750, 1990.

[36] A.K. Gupta, D.G. Lilley, and N. Syred. Swirl flows. Abacus Press, 1984.

[37] E.R. Hawkes and R.S. Cant. A flame surface density approach to large-eddy simulation of premixed turbulent combustion. Proceedings of the Combustion Institute, 28:51-58, 2000.

[38] M.A. Heckl. Active control of the noise from a Rijke tube. Journal of Sound and Vibration, 124(1):117-133, 1988.

[39] S. Hermeth, G. Staffelbach, L. Gicquel, T. Poinsot, H.J. Krediet, and W. Krebs. LES and Flame Transfer Function measurement in a perfectly premixed swirled burner. INCA Conference, Toulouse, 2011.

[40] I. Hernandez, B. Cuenot, and T. Poinsot. LES and acoustic analysis of thermoacoustic instabilities in a partially premixed model combustor. INCA Conference, Toulouse, 2011. 
[41] P.A. Hield, M.J.Brear, and S.H. Jin. Thermo-acoustic limit cycles in a premixed laboratory combustor with open and choked exits. Combustion and Flame, 156:1683-1697, 2009.

[42] S. Hubbard and A.P. Dowling. Acoustic Instabilities in Premix Burners. AIAA Paper 98-2272, 1998.

[43] S. Hubbard and A.P. Dowling. Acoustic resonances of an industrial gas turbine combustion system. Journal of Engineering for Gas Turbines and Power, 123(4):766773, 2001.

[44] A. Huber. Impact of fuel supply impedance and fuel staging on gas turbine combustion stability. PhD thesis, Technische Universität München, Lehrstuhl für Thermodynamik, 2009.

[45] I. Hurle, R. Price, T. Sugden, and A. Thomas. Sound emission from open turbulent premixed flames. Proceedings of Royal Society, 303:409-427, 1968.

[46] H. Jasak. Error analysis and estimation in the Finite Volume method with applications to fluid flows. PhD thesis, Imperial College, University of London, 1996.

[47] J.F. van Kampen. Acoustic pressure oscillations induced by confined turbulent premixed natural gas flames. PhD thesis, University of Twente, 2006.

[48] K.T. Kim, H.J. Lee, J.G. Lee, B.D. Quay, and D.A. Santavicca. Flame transfer function measurement and instability frequency prediction using a thermo acoustic model. Proceedings ASME Turbo Expo, Orlando, GT2009-60026, 2009.

[49] K.T. Kim, J.G. Lee, B.D. Quay, and D.A. Santavicca. Spatially distributed flame transfer functions for predicting combustion dynamics in lean premixed gas turbine combustors. Combustion and Flame, 157:1718-1730, 2010.

[50] E. Knudsen and H. Pitsch. Large-Eddy Simulation for Combustion Systems: Modeling Approaches for Partially Premixed Flows. The Open Thermodynamic Journal, 4:76-85, 2010.

[51] K. Kostrzewa. Advanced computational methods in identification of thermo-acoustic systems. PhD thesis, University of Stuttgart, 2009.

[52] W. Krebs. Thermoacoustic Design Tools and Passive Control: Siemens Power Generation Approaches. Progress in Astronautics and Aeronautics, AIAA, 210:89112, 2010.

[53] W. Krebs, P. Flohr, B. Prade, and S. Hoffmann. Thermoacoustic Stability Chart for High Intense Gas Turbine combustion Systems. Combustion Science and Technology, 174:99-128, 2002.

[54] W. Krebs, G. Walz, and S. Hoffmann. Thermoacoustic analysis of an annular combustor. $5^{\text {th }}$ AIAA Aeroacoustics Conference, AIAA 99-1971, 1999. 
[55] H.J. Krediet, C.H. Beck, W. Krebs, J.B.W. Kok, S. Schimek, and C.O. Paschereit. Identification of the Flame Describing Function of a premixed swirl flame from LES. $7^{\text {th }}$ Mediterranean Combustion Symposium, Chia Laguna, Sardinia, Italy, 2011.

[56] H.J. Krediet, J.E. Portillo, W. Krebs, and J.B.W. Kok. Prediction of Thermoacoustic Limit Cycles during Premixed Combustion using the Modified Galerkin Approach. 46 $6^{\text {th }}$ AIAA/ASME/SAE/ASEE Joint Propulsion Conference and Exhibit, Nashville, 2010.

[57] U. Krüger, J. Hüren, S. Hoffmann, W. Krebs, P. Flohr, and D. Bohn. Prediction and Measurement of Thermoacoustic Improvements in Gas Turbines with Annular Combustion Systems. Journal of Engineering for Gas turbines and Power, 123:557, 2001.

[58] M. Lauer. Determination of the Heat Release Distribution in Turbulent Flames by Chemiluminescence Imaging. PhD thesis, Technische Universität München, Lehrstuhl für Thermodynamik, 2011.

[59] J. Lee and D. Santavicca. Experimental diagnostics for the study of combustion instabilities in lean premixed combustors. Journal of Propulsion and Power, 19(5):735-750, 2003.

[60] T. Lieuwen. Investigation of combustion instability mechanisms in premixed gas turbines. PhD thesis, Georgia Institute of Technology, 1999.

[61] T. Lieuwen, B.D. Bellows, M.K. Bobba, A. Forte, and J.M. Seitzman. Flame transfer function saturation mechanisms in a swirl stabilized combustor. Transactions of the ASME, 129:954-961, 2007.

[62] T. Lieuwen, B.D. Bellows, M.K. Bobba, and J.M. Seitzman. Nonlinear Flame transfer function characteristics in a swirl stabilized combustor. Journal of Engineering for Gas Turbines and Power, 129:954-961, 2007.

[63] T. Lieuwen and Y. Neumeier. Nonlinear pressure-heat release transfer function measurements in a premixed combustor. Proceedings of the Combustion Institute, 29:99-105, 2002.

[64] L. Ljung. System Identification Theory for the User. PTR Prentice Hall Information and System Series, 1999.

[65] The Mathworks. Matlab reference guide.

[66] K.R. McManus, T. Poinsot, and S.M. Candel. A review of active control of combustion instabilities. Prog. Energy Combustion Science, 19:1-29, 1993.

[67] M.L. Munjal. Acoustics of ducts and mufflers with application to exhaust and ventilation systems design. John Wiley \& Sons Inc., 1945.

[68] S. Nagaraja, K. Kedia, and R.I. Sujith. Characterizing energy growth during combustion instabilities: Singular values or Eigen values? Proceedings of the Combustion Institute, 32:2933-2940, 2009. 
[69] F. Nicoud and T. Poinsot. Thermoacoustic instabilities: Should the Rayleigh criterion be extended to include entropy changes? Combustion and Flame, 142:153159, 2005.

[70] F. Nicoud and T. Poinsot. Acoustic Modes in Combustors with Complex Impedances and Multidimensional Active Flames. AIAA Journal, 45(2):426-441, 2007.

[71] P. Palies, D. Durox, T. Schuller, and S. Candel. The combined dynamics of swirler and turbulent premixed swirling flames. Combustion and Flame, 157:1698-1717, 2010.

[72] P. Palies, D. Durox, T. Schuller, and S. Candel. Nonlinear combustion instability analysis based on the Flame Describing Function applied to turbulent premixed swirling flames. Combustion and Flame, 158:1980-1991, 2011.

[73] C. Pankiewitz, A. Fischer, C. Hirsch, and T. Sattelmayer. Computation of Transfer Matrices for Gas Turbine Combustors including Acoustics/Flame Interaction. 9th AIAA/CEAS Area-acoustics Conference and Exhibit, 2003.

[74] C. Pankiewitz and T. Sattelmayer. Time domain simulation of combustion instabilities in annular combustors. Journal of Engineering for Gas Turbines and Power, 125(3):677-685, 2003.

[75] T. Poinsot and D. Veynante. Theoretical and Numerical Combustion. Edwards, Philadelphia, second edition, 2005.

[76] W. Polifke. Numerical Techniques for identification of Acoustic Multi-Ports. VKI Lecture Series, 2004.

[77] W. Polifke, A. Poncet, C.O. Paschereit, and K. Döbbeling. Reconstruction of acoustic transfer matrices by instationary computational fluid dynamics. Journal of Sound and Vibration, 245:483-510, 2001.

[78] W. Polifke and C. Wall. Non-reflecting boundary conditions for acoustic transfer matrix estimation with LES. Center for Turbulence Research Proceedings of the Summer Program, 2002.

[79] S.B. Pope. PDF methods for turbulent reactive flows. Energy Combustion Science, 11:119-192, 1985.

[80] S.B. Pope. Turbulent Flows. Cambridge University Press, 2000.

[81] J.E. Portillo, J.C. Sisco, W.E. Anderson, and V. Sankaran. Application of a generalized instability model to a longitudinal mode combustion chamber. $43^{\text {rd }}$ AIAA/ASME/SAE/ASEE Joint Propulsion Conference and Exhibit, Cincinatti, 2007.

[82] J.E. Portillo, J.C. Sisco, M.J. Corless, W.E. Anderson, and V. Sankaran. Generalized Combustion Instability Model. 42 ${ }^{\text {nd }}$ AIAA/ASME/SAE/ASEE Joint Propulsion Conference and Exhibit, Sacramento, 2006. 
[83] J.W.S. Rayleigh. The Explanation of certain Acoustical Phenomena. Nature, XVIII:319-321, 1878.

[84] P.L. Rijke. Notiz über eine neue Art, die in einer an beiden Enden offenen Röhre enthaltene Luft in Schwingungen zu versetzen. Annalen der Physik, 183:339-343, 1859.

[85] S. Schimek, J.P. Moeck, and C.O. Paschereit. Design of a combustion test rig with high amplitude forcing capabilities for nonlinear flame transfer function measurements. 16th Int. Congress on Sound and Vibration (ICSV16), 2009.

[86] S. Schimek, J.P. Moeck, and C.O. Paschereit. An experimental investigation of the nonlinear response of an atmospheric swirl-stabilized premixed flame. ASME Turbo Expo, GT2010-22827, 2010.

[87] S. Schimek, J.P. Moeck, and C.O. Paschereit. Experimental investigation of the influence of high amplitude forcing and swirl fluctuations on the flow field and the transfer function of a swirl-stabilized flame. $47^{\text {th }}$ AIAA/ASME/SAE/ASEE Joint Propulsion Conference and Exhibit, San Diego, 2010.

[88] B. Schuermans, V. Belluci, F. Guethe, F. Meili, P. Flohr, and C.O. Paschereit. A detailed analysis of thermoacoustic interaction mechanisms in a turbulent premixed flame. ASME Turbo Expo, GT2004-53831, 2004.

[89] B. Schuermans, V. Belluci, D. Novak, and C.O. Paschereit. Modeling of Complex Thermoacoustic Systems: A State-Space Approach. Ninth International Congress of Sound and Vibration, 2002.

[90] F. Selimefendigil and W. Polifke. A nonlinear frequency domain model for limit cycles in thermoacoustic systems with modal coupling. International Journal of Spray and Combustion Dynamics, 3:303-330, 2011.

[91] L. Selle, L. Benoit, T. Poinsot, F. Nicoud, and W. Krebs. Joint use of Compressible Large-Eddy Simulation and Helmholtz Solvers for the Analysis of Rotating Modes in an Industrial Burner. Combustion and Flame, 145:194-205, 2006.

[92] J.C. Sisco, J.E. Portillo, Y.C. Yu, and W.E. Anderson. Nonlinear characteristics of longitudinal instabilities in a model rocket combustor. $43^{\text {rd }}$ AIAA/ASME/SAE/ASEE Joint Propulsion Conference and Exhibit, Cincinatti, 2007.

[93] J. Smagorinsky. General Circulation Experiments with the Primitive Equations. Monthly Weather Review, 91:99-164, 1963.

[94] J.A. van Oijen, F.A. Lammers, and L.P.H. de Goey. Modeling of complex premixed burner systems by using flamelet-generated manifolds. Combustion and Flame, 127:2124-2134, 2001.

[95] Y.C. Yu, J.C. Sisco, C.L. Merkle, W.E. Anderson, and V. Sankaran. The Examination of Spatial Mode Shapes and Resonant Frequencies Using Linearized Euler Solutions. $37^{\text {th }}$ AIAA Fluid Dynamics Conference and Exhibit (Miami, FL), AIAA Paper 2007-3999, 2007. 
[96] S.W. Yuen, A.M.G. Gentemann, and W. Polifke. Investigation of the Influence of Boundary Conditions on System Identifiability using Real Time System Modeling. 11th Int. Congress on Sound and Vibration (ICSV11), pages 3501-3508, 2004.

[97] V.L. Zimont and A.N. Lipatnikov. A numerical model of premixed turbulent combustion of gases. Chemical Physics Reports, 14(7):993-1025, 1995. 


\section{Acknowledgements}

Finally I am writing the last page of my dissertation, the result of the past three and a half years work. On this page I will try to thank everyone who helped me writing this dissertation or was for other reasons important to me.

Theo and Jim for giving me the trust to start a Phd, bringing me in contact with Siemens and the Limousine Project. I am very grateful for the many feedback you gave me during your visits to Germany or my visits to Twente.

Werner for allowing me to start my Phd at Siemens and being my daily supervisor. Your critical views helped me stay sharp and definitely improved my work a lot. During our many conversations we often tried to convince each other by talking louder than the other, since the one who talks loudest obviously has the best arguments. Our colleagues often had to use earplugs when we met, to keep the noise at a reasonable level. At one moment they even installed a sound sensitive traffic light at our corner, that frequently started blinking red during the heat of our discussions. Besides gas turbines, we have a common interest, which is running, it is pity I havent beaten your half marathon time yet.

Of course there are also many other Siemens colleagues I need to thank. Christian and Enrique were also closely involved within my work. Thanks to Christians work on the CFD code and Enrique's work on the thermo-acoustic codes, I already had a lot of tools to start with, accelerating my work a lot. You always made time for to help me out with many problems.

Special thanks also go to Peter, Rajesh, Mathias, Stephan, Bhedad and other colleagues I forgot to mention for your suggestions and/or after work beers.

My work was done within a larger European project. Id like to thank the other PhD students of the Limousine Project as well: Patrick, Bela, Thomas, Ignacio, Basti, Lukas, Sebastian, Simone, Antonio, Roel, Salvo, Juan Carlos, Can, Mehmet, Santosh, Mina and Reddy. The Limousine Meetings in Twente, Toulouse, Keele, Munich, Zaragoza and London were great fun and I will certainly miss the city trips, making fun about each other's countries and the many evening beers!

All my friends: my German friends from the Mulheim area, my Dutch friends from Veenendaal, my friends group from my study period in Enschede known as the KZW, and my former running buddies from DAV Kronos. Thanks for the many activities, holidays and nights out into town.

At last my family for supporting me and giving me the chance to study. I hope that now after about three years you have a good idea what I did the past three years.

Harmen Krediet

Zwolle, May 2012 
